\title{
Aerobic Copper-Catalyzed Intramolecular Cascade Oxidative Isomerization/[4+4] Cyclization of 2,2'-Disubstituted Stilbenes
}

Zhi-Jun Zhang a, ${ }^{\text {, }}$, Xu Zhou ${ }^{\text {a, }}$, Dashan Li ${ }^{\text {b }}$, Yang Chen ${ }^{\text {b }}$, Wen-Wen Xiao ${ }^{\text {b }}$, Rong-Tao Li a, ${ }^{\text {a }}$, and Li-Dong Shao ${ }^{b, *}$

${ }^{a}$ Faculty of Life Science and Technology, Kunming University of Science and Technology, Kunming, 650500, China.

${ }^{b}$ Yunnan Key Laboratory of Southern Medicinal Utilization, School of Chinese Materia Medica, Yunnan University of Chinese Medicine,

Kunming, 650500, China.

*Corresponding author: rongtaolikm@163.com (R.-T. L); shaolidong@ynutcm.edu.cn (L.-D. S)

\section{Table of contents}

\begin{tabular}{ll}
\hline \multicolumn{1}{c}{ Contents } & Pages \\
\hline Scheme S1. Synthesis of 2,2'-disubstituted stilbenes & $\mathrm{S} 1$ \\
Figure S1. Structures of 1a-1ai & $\mathrm{S} 2$ \\
Table S1. Screening of Cu sources and ligands & $\mathrm{S} 3$ \\
Figure S2. Online UV-Vis detection of CuCl/DBED-catalyzed oxidative cyclization of 1a & $\mathrm{S} 4$ \\
Figure S3. Online ${ }^{13} \mathrm{C}-\mathrm{NMR} / \mathrm{DEPT} 135^{\circ}(150 \mathrm{MHz})$ analysis of CuCl/DBED-catalyzed & $\mathrm{S} 5$ \\
oxidative cyclization of 1a in DMF- $d 7$ & \\
X-ray crystal structure analysis for compounds $\mathbf{2 a}, \mathbf{2 n}, \mathbf{2 t}$, and 2am & $\mathrm{S} 6-10$ \\
Details for determination of KIE values & $\mathrm{S} 11-13$ \\
Computational details & $\mathrm{S} 14-22$ \\
Reference & $\mathrm{S} 23$ \\
NMR spectra copies for all synthetic compounds & $\mathrm{S} 24$ \\
${ }^{1} \mathrm{H}$ and ${ }^{13} \mathrm{C}$ NMR spectra of 1a-1an & $\mathrm{S} 25-75$ \\
${ }^{1} \mathrm{H}$ and ${ }^{13} \mathrm{C}$ NMR spectra of 2a-2an & $\mathrm{S} 76-132$ \\
\hline
\end{tabular}




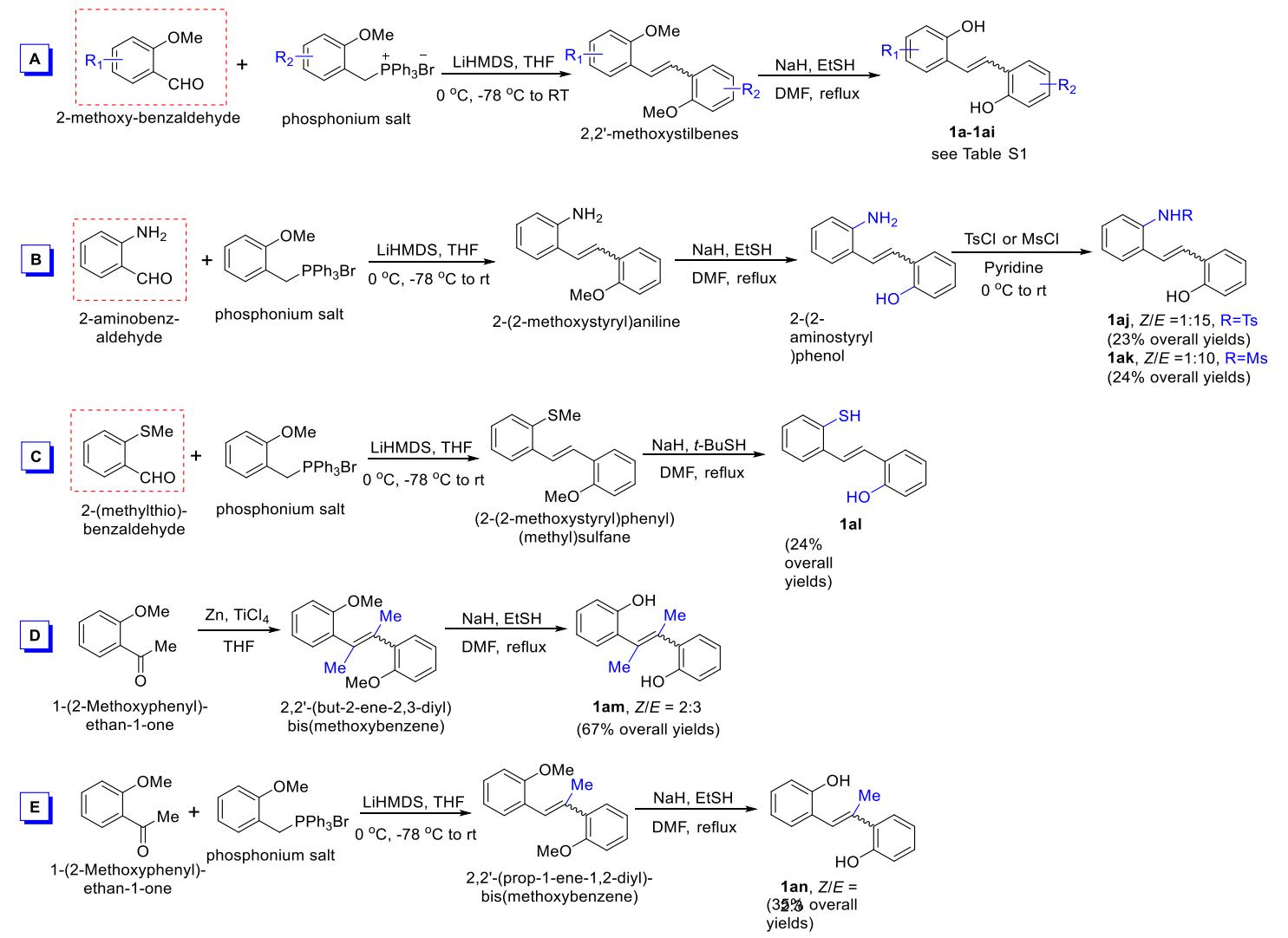

Scheme S1. Synthesis of 2,2'-disubstituted stilbenes. 
<smiles>Oc1ccccc1/C=C/c1ccccc1O</smiles>

1a (45\%)<smiles>[R]c1ccc(/C=C/c2ccccc2O)c(O)c1</smiles>

1f, $R_{1}=$ Me $(60 \%)$

$\mathbf{1 g}, \mathrm{R}_{1}=\mathrm{SEt}(\mathrm{Z} / \mathrm{E}, 1: 4,54 \%)$

$1 \mathrm{~h}, \mathrm{R}_{1}=\mathrm{F}(\mathrm{Z} / \mathrm{E}, 2: 3,65 \%)$<smiles>Oc1ccc(Cl)cc1/C=C/c1cc(Cl)ccc1O</smiles>

$10(Z / E, 5: 2,68 \%)$<smiles>[R]c1ccc(/C=C/c2cccc(F)c2O)c(O)c1</smiles>

$1 \mathrm{u}, \mathrm{R}_{1}=\mathrm{Cl}(Z / E, 3: 4,67 \%)$

$1 v, \mathrm{R}_{1}=\mathrm{CN}(59 \%)$

$1 w, R_{1}=M e(Z / E, 1: 4,62 \%)$

$1 \mathrm{x}, \mathrm{R}_{1}=\mathrm{F}(53 \%)$<smiles>Oc1ccccc1/C=C/c1cc(Cl)ccc1O</smiles>

$1 \mathrm{~b}(54 \%)$<smiles>Oc1ccccc1/C=C/c1cccc(F)c1O</smiles>

1c $(53 \%)$<smiles>[R]c1ccc(O)c(/C=C/c2ccccc2O)c1</smiles>

1d, $R_{1}=\operatorname{PPr}(38 \%)$ $1 e, R_{1}=t B u(43 \%)$<smiles>[R2]c1ccc(/C=C/c2ccc(C)cc2O)c(O)c1</smiles>

1i, $\mathrm{R}_{2}=\mathrm{Me}(57 \%)$

1j, $R_{2}=C N(Z \mid E, 1: 7,19 \%)$<smiles>Oc1c(F)cccc1/C=C/c1cccc(P)c1O</smiles>

1p (ZIE, 2:1, 57\%)<smiles>Cc1ccc(/C=C/c2cc(Cl)ccc2O)c(O)c1</smiles>

1y (ZIE, 2:1, 74\%)<smiles>Oc1ccccc1/C=C/c1cccc(O)c1O</smiles>

$1 \mathrm{ab}(25 \%)$<smiles>[R]c1ccc(O)c(/C=C/c2cc(C(C)C)ccc2O)c1</smiles>

$1 \mathrm{k}, \mathrm{R}_{1}=\operatorname{PPr}(41 \%)$

1I, $R_{1}=$ tBu $(78 \%)$

$1 \mathrm{~m}, \mathrm{R}_{1}=\mathrm{Cl}(\mathrm{Z} / \mathrm{E}, 1: 3,35 \%)$<smiles>[R]c1ccc(O)c(/C=C/c2cccc(F)c2O)c1</smiles>

1q, $R_{1}=\operatorname{Pr}(36 \%)$

$1 \mathbf{r}, R_{1}=t B u(27 \%)$

1s, $\mathrm{R}_{1}=\mathrm{Cl}(\mathrm{Z} / \mathrm{E}, 1: 2,82 \%)$<smiles>[R]c1ccc(O)c(/C=C/c2ccc(C#N)cc2O)c1</smiles>

1z, $R_{1}=\operatorname{Pr}(Z / E, 1: 10,70 \%)$ 1aa, $R_{1}=\mathrm{Cl}(Z I E, 1: 15,50 \%)$

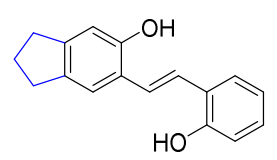<smiles>Oc1ccc2ccccc2c1/C=C/c1c(O)ccc2ccccc12</smiles>

1n (14\%)<smiles>CC(C)c1ccc(O)c(C=Cc2ccc(Cl)cc2O)c1</smiles>

1t (ZIE, 1:7, 41\%)<smiles>[R2]c1ccc(O)c(/C=C/c2c(O)ccc3ccccc23)c1</smiles>

1ad, $\mathrm{R}_{2}=\mathrm{H}(80 \%)$

$1 \mathrm{ae}, \mathrm{R}_{2}=5 "-\mathrm{BBu}(45 \%)$

1af, $R_{2}=4 "-C N(Z / E, 1: 9,43 \%)$

$1 \mathrm{ag}, \mathrm{R}_{2}=5 "-\operatorname{Pr}(Z / E, 2: 5,36 \%)$

1ah, $R_{2}=5 "-C l(Z / E, 1: 18,38 \%)$

1ai, $R_{2}=3 "-F(51 \%)$

Figure S1. Structures of 1a-1ai (overall yields from 2-methoxy benzaldehyde) 
Table S1. Screening of $\mathrm{Cu}$ sources and ligands ${ }^{a}$

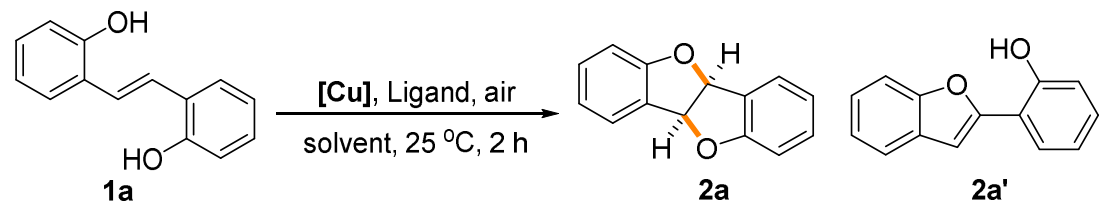

\begin{tabular}{|c|c|c|c|c|c|}
\hline Entry & {$[\mathbf{C u}](\mathrm{mol} \%)$} & Ligand (mol \%) & Additive & Solvent & Yield 2a/2a' (\%) ${ }^{b}$ \\
\hline 1 & $\mathrm{Cu}(\mathrm{OTf})_{2}(10)$ & - & - & DMF & $\mathrm{NR}^{c}$ \\
\hline 2 & $\mathrm{Cu}(\mathrm{OTf})_{2}(10)$ & $\operatorname{DBED}(10)$ & - & DMF & $65 / 25$ \\
\hline 3 & $\mathrm{Cu}(\mathrm{OH})_{2}(10)$ & DBED (10) & - & DMF & $23 / 30$ \\
\hline 4 & $\mathrm{CuSO}_{4}(10)$ & $\operatorname{DBED}(10)$ & - & DMF & $42 / 21$ \\
\hline 5 & $\mathrm{Cu}(\mathrm{OAc})_{2}(10)$ & $\operatorname{DBED}(10)$ & - & DMF & $30 / 40$ \\
\hline 6 & $\mathrm{Cu}(\mathrm{acac})_{2}(10)$ & DBED (10) & - & DMF & $34 / 40$ \\
\hline 7 & $\mathrm{Cu}\left(\mathrm{CH}_{3} \mathrm{CN}\right)_{4} \mathrm{PF}_{6}(10)$ & DBED (10) & - & DMF & $50 / 10$ \\
\hline 8 & $\mathrm{CuI}(10)$ & DBED (10) & - & DMF & $46 / 18$ \\
\hline 9 & $\mathrm{CuCl}(10)$ & DBED (10) & - & DMF & $85 / 0$ \\
\hline 10 & $\mathrm{CuCl}(10)$ & $\operatorname{DBED}(10)$ & - & DMSO & $42 / 0$ \\
\hline 11 & $\mathrm{CuCl}(10)$ & $\operatorname{DBED}(10)$ & - & THF & $55 / 0$ \\
\hline 12 & $\mathrm{CuCl}(10)$ & DBED (10) & - & $\mathrm{MeCN}$ & $17 / 17$ \\
\hline 13 & $\mathrm{CuCl}(10)$ & DBED (10) & - & Toluene & $25 / 5$ \\
\hline 14 & $\mathrm{CuCl}(10)$ & DBED (10) & - & $\mathrm{MeOH}$ & $28 / 14$ \\
\hline 15 & $\mathrm{CuCl}(10)$ & $\operatorname{DBED}(10)$ & - & $t \mathrm{BuOH}$ & $15 / 10$ \\
\hline 16 & $\mathrm{CuCl}(10)$ & TMEDA (10) & - & DMF & $82 / 0$ \\
\hline $17^{d}$ & $\mathrm{CuCl}(10)$ & $\operatorname{EDA}(10)$ & - & DMF & trace \\
\hline $18^{d}$ & $\mathrm{CuCl}(10)$ & PPA (10) & - & DMF & $10 / 32$ \\
\hline $19^{d}$ & $\mathrm{CuCl}(10)$ & MPPA (10) & - & DMF & $15 / 25$ \\
\hline $20^{d}$ & $\mathrm{CuCl}(10)$ & PYR (10) & - & DMF & $18 / 41$ \\
\hline $21^{d}$ & $\mathrm{CuCl}(10)$ & $\operatorname{DCA}(10)$ & - & DMF & trace \\
\hline $22^{d}$ & $\mathrm{CuCl}(10)$ & DABOC (10) & - & DMF & $8 / 30$ \\
\hline $23^{d}$ & $\mathrm{CuCl}(10)$ & DIPEA (10) & - & DMF & trace \\
\hline 24 & $\mathrm{CuCl}(5)$ & DBED (5) & - & DMF & $86 / 0$ \\
\hline 25 & $\mathrm{CuCl}(15)$ & DBED (15) & - & DMF & $89 / 0$ \\
\hline 26 & $\mathrm{CuCl}(20)$ & DBED (20) & - & DMF & $80 / 0$ \\
\hline 27 & $\mathrm{CuCl}(5)$ & DBED (5) & $\mathrm{H}_{2} \mathrm{O}(100 \mathrm{~mol} \%)$ & DMF & $83 / 7$ \\
\hline 28 & $\mathrm{CuCl}(5)$ & DBED (5) & $4 \AA$ MS (100 wt\%) & DMF & $95 / 0$ \\
\hline
\end{tabular}

${ }^{a}$ Unless otherwise noted, all of the reactions were performed open to air in anhydrous solvents $(1 \mathrm{~mL})$ with $1 \mathbf{a}(0.5 \mathrm{mmol})$ at $25{ }^{\circ} \mathrm{C}$ for 2 h. ${ }^{b}$ Isolated yield; ${ }^{c} \mathrm{NR}$ : no reaction ; ${ }^{d}$ Recovery of starting materials $\mathbf{1 a}$

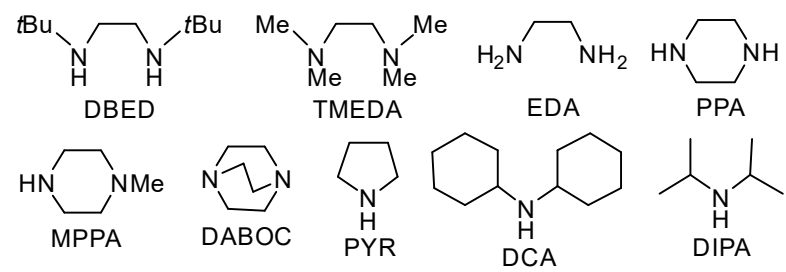




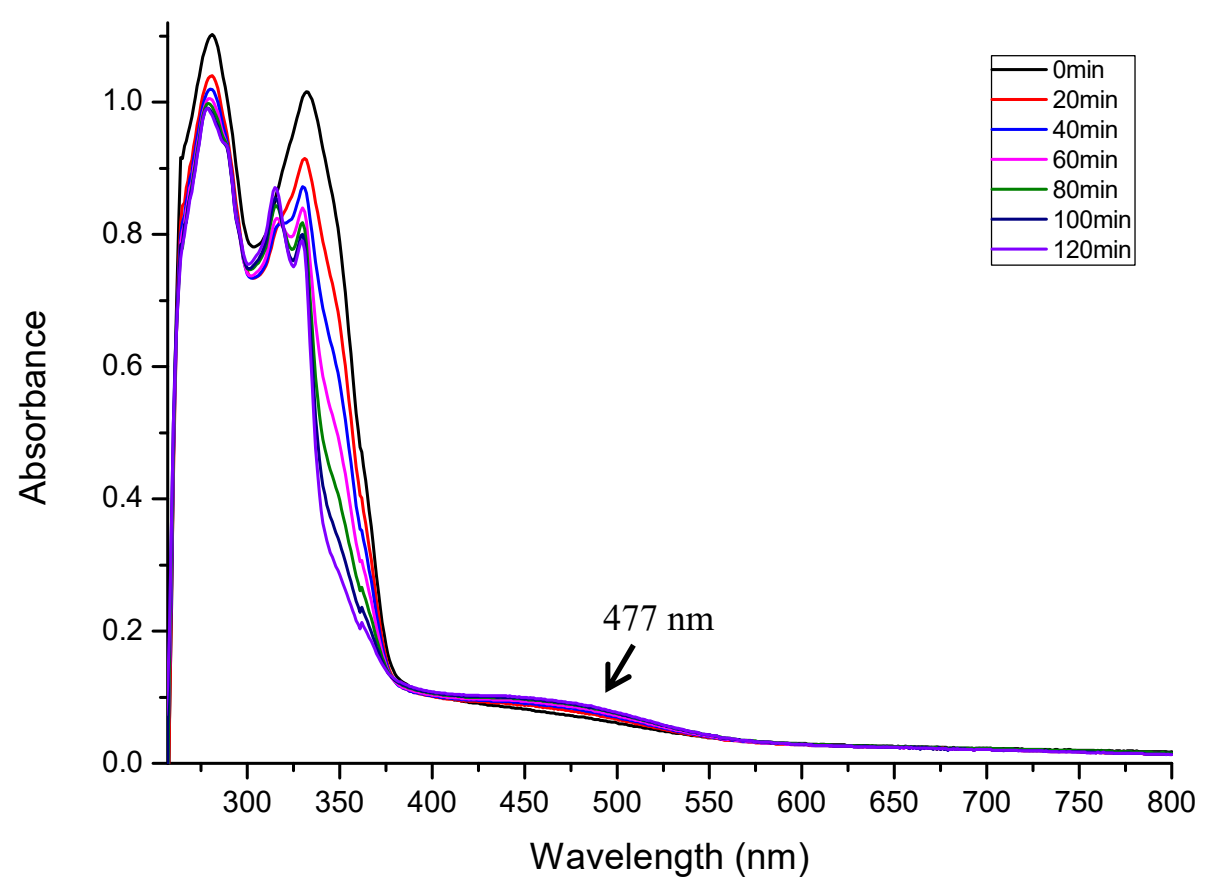

Figure S2. Online UV-Vis detection of $\mathrm{CuCl} / \mathrm{DBED}$-catalyzed oxidative cyclization of 1a $(0.01 \mathrm{mg} / \mathrm{mL})$ in DMF. 


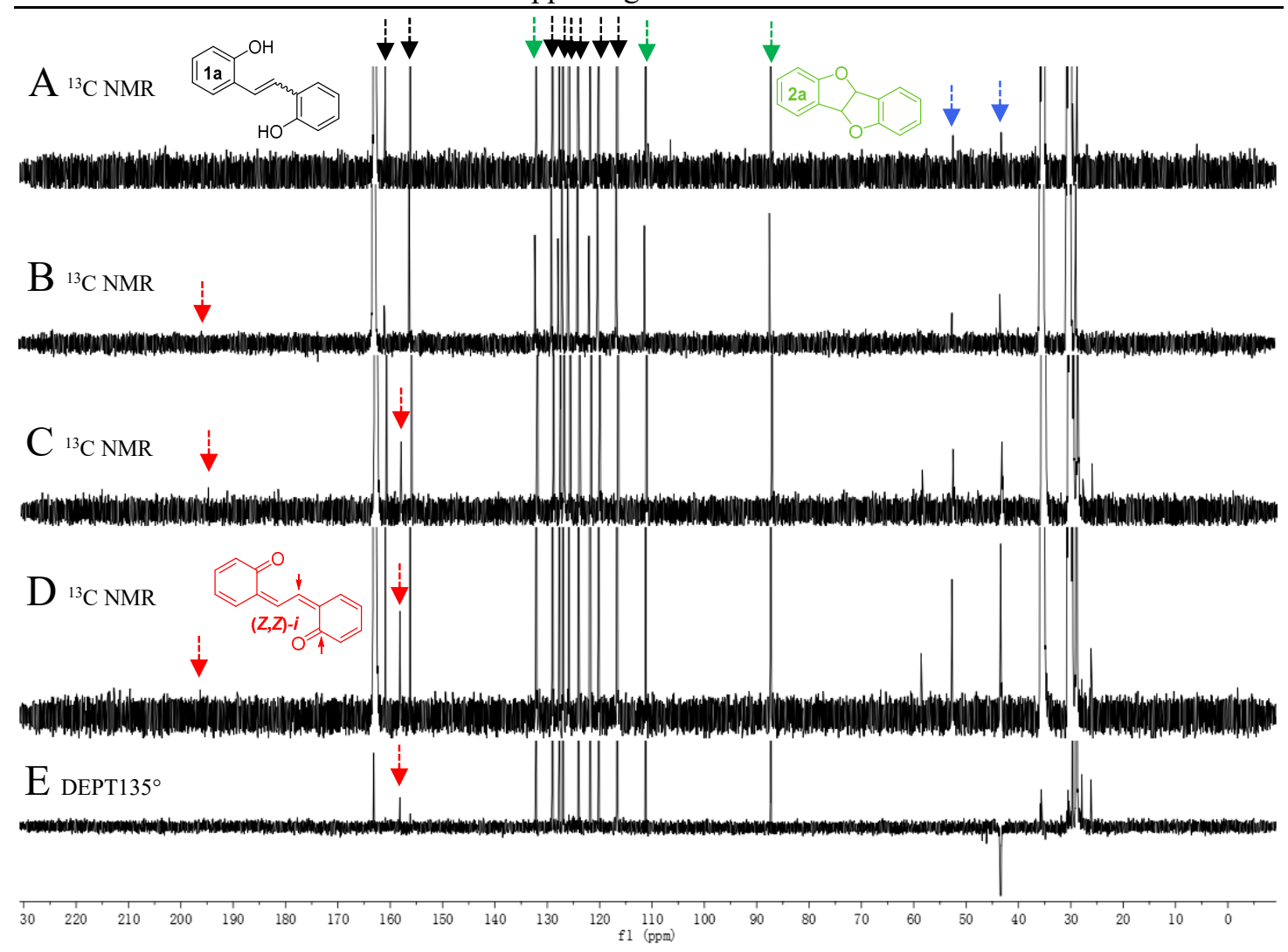

Figure S3. Online ${ }^{13} \mathrm{C}-\mathrm{NMR} / \mathrm{DEPT} 135^{\circ}(150 \mathrm{MHz})$ analysis of $\mathrm{CuCl} / \mathrm{DBED}$-catalyzed oxidative cyclization of 1a in DMF- $d_{7}$. Arrows represent signals from substrate 1a (black), product 2a (green), catalyst (blue), and intermediate (Z,Z)-i (red). (A) $5 \mathrm{~mol} \%$ of CuCl/DBED (5min); (B) 5 mol\% of $\mathrm{CuCl} / \mathrm{DBED}$ (10min after blow with air); (C) $50 \mathrm{~mol} \%$ of $\mathrm{CuCl} / \mathrm{DBED}$ (15min after blow with air). (D) $50 \mathrm{~mol} \%$ of pre-prepared DBED $\cdot \mathrm{Cu}^{\mathrm{II}} \mathrm{OH}$ complex (10min). (E) $50 \mathrm{~mol} \%$ of $\mathrm{CuCl} / \mathrm{DBED}$ (15min after blow with air). 


\section{Xray crystal structure analysis for compounds 2a, 2n, 2t, and 2am}

Colorless crystals of compounds were obtained in toluene/methanol (9:1). The intensity data for compounds were collected on a Bruker APEX DUO diffractometer equipped with an APEX II CCD using $\mathrm{Cu} \mathrm{K} \alpha$ radiation (100 K). Cell refinement and data reduction were performed with a Bruker SAINT. The structures were solved by direct methods using SHELXS-97.28 Refinements were carried out with SHELXL-97 using full-matrix least-squares, with anisotropic displacement parameters used for all non-hydrogen atoms. The hydrogen atoms were placed at calculated positions and refined using a riding model. Molecular graphics were computed using PLATON.

Crystal data for 2a (CCDC 2038542): $\mathrm{C}_{14} \mathrm{H}_{10} \mathrm{O}_{2}, M=210.22, a=8.8270(2) \AA, b=$ 10.3033(2) $\AA, c=10.9546(3) \AA, \alpha=90^{\circ}, \beta=90^{\circ}, \gamma=90^{\circ}, V=996.29(4) \AA^{3}, T=100$.(2) K, space group $P 212121, Z=4, \mu(\mathrm{Cu} \mathrm{K \alpha})=0.752 \mathrm{~mm}^{-1}$, 7188 reflections measured, 1941 independent reflections $\left(R_{\text {int }}=0.0339\right)$. The final $R_{l}$ values were $0.0294(I>2 \sigma(I))$. The final $w R\left(F^{2}\right)$ values were $0.0739(I>2 \sigma(I))$. The final $R_{l}$ values were 0.0306 (all data). The final $w R\left(F^{2}\right)$ values were 0.0748 (all data). The goodness of fit on $F^{2}$ was 1.082. Flack parameter $=-0.03(12)$.

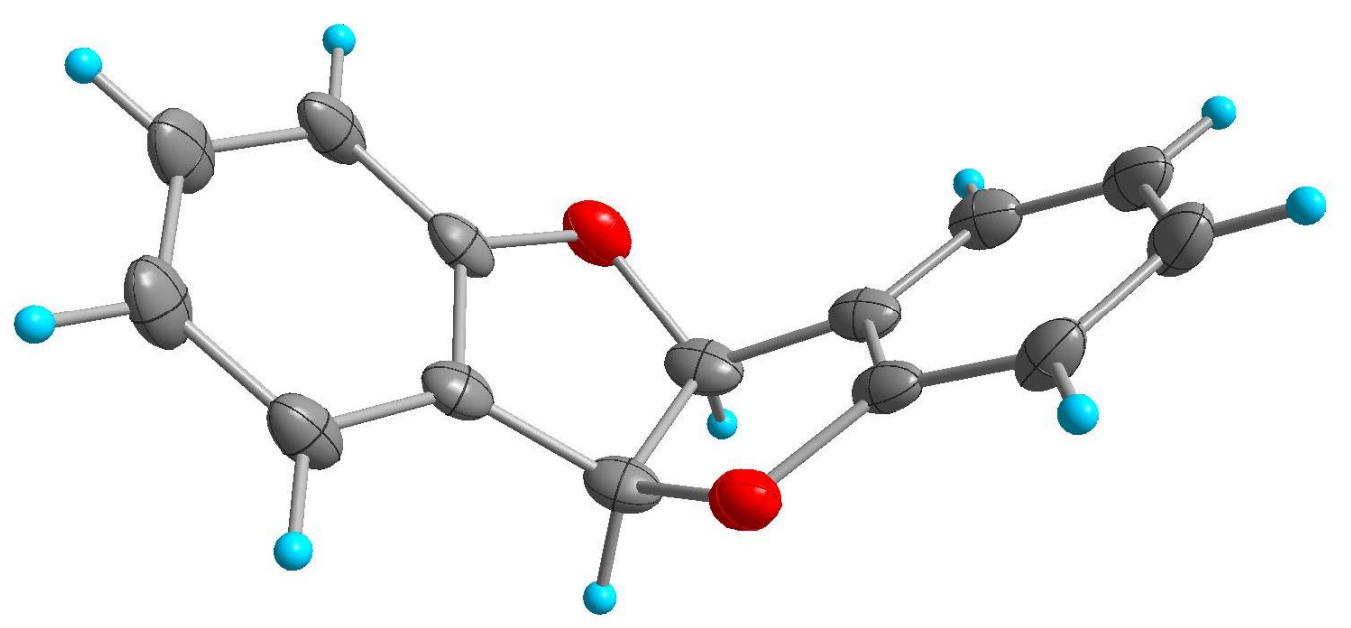

X-ray ORTEP drawing of compound 2a shown as 30\% probability. 


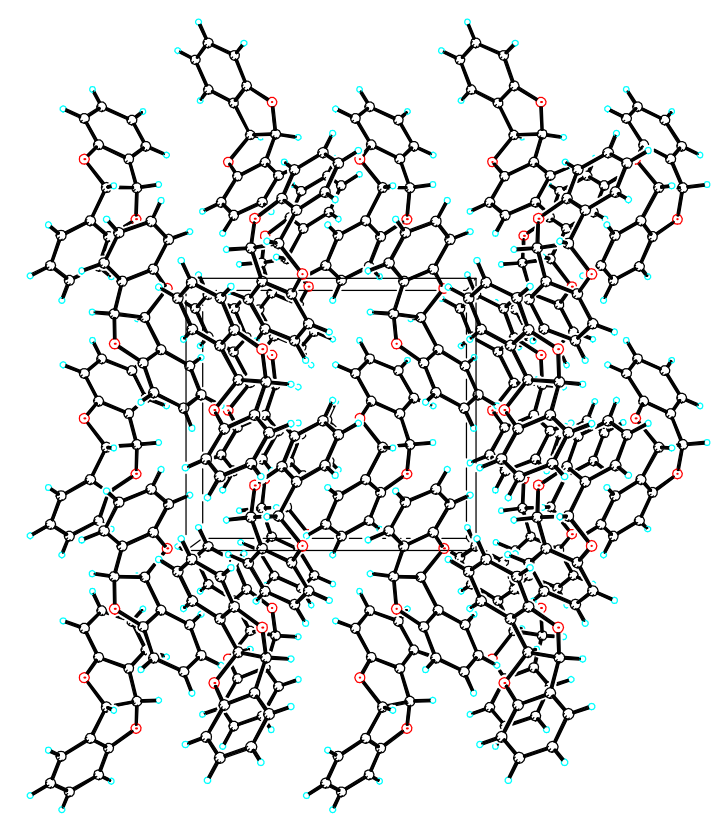

View of the pack drawing of $\mathbf{2 a}$.

Crystal data for 2n (CCDC 2038540): $\mathrm{C}_{22} \mathrm{H}_{14} \mathrm{O}_{2}, M=310.33, a=14.0351(6) \AA, b=4.0170(2)$ $\AA, c=13.2658(6) \AA, \alpha=90^{\circ}, \beta=103.174(2)^{\circ}, \gamma=90^{\circ}, V=728.23(6) \AA^{3}, T=100 .(2) \mathrm{K}$, space group $P 12 / c 1, Z=2, \mu(\mathrm{Cu} \mathrm{K \alpha})=0.713 \mathrm{~mm}^{-1}, 6005$ reflections measured, 1411 independent reflections $\left(R_{\text {int }}=0.0616\right)$. The final $R_{I}$ values were $0.0661(I>2 \sigma(I))$. The final $w R\left(F^{2}\right)$ values were $0.1728(I>2 \sigma(I))$. The final $R_{l}$ values were 0.0687 (all data). The final $w R\left(F^{2}\right)$ values were 0.1780 (all data). The goodness of fit on $F^{2}$ was 1.071 .

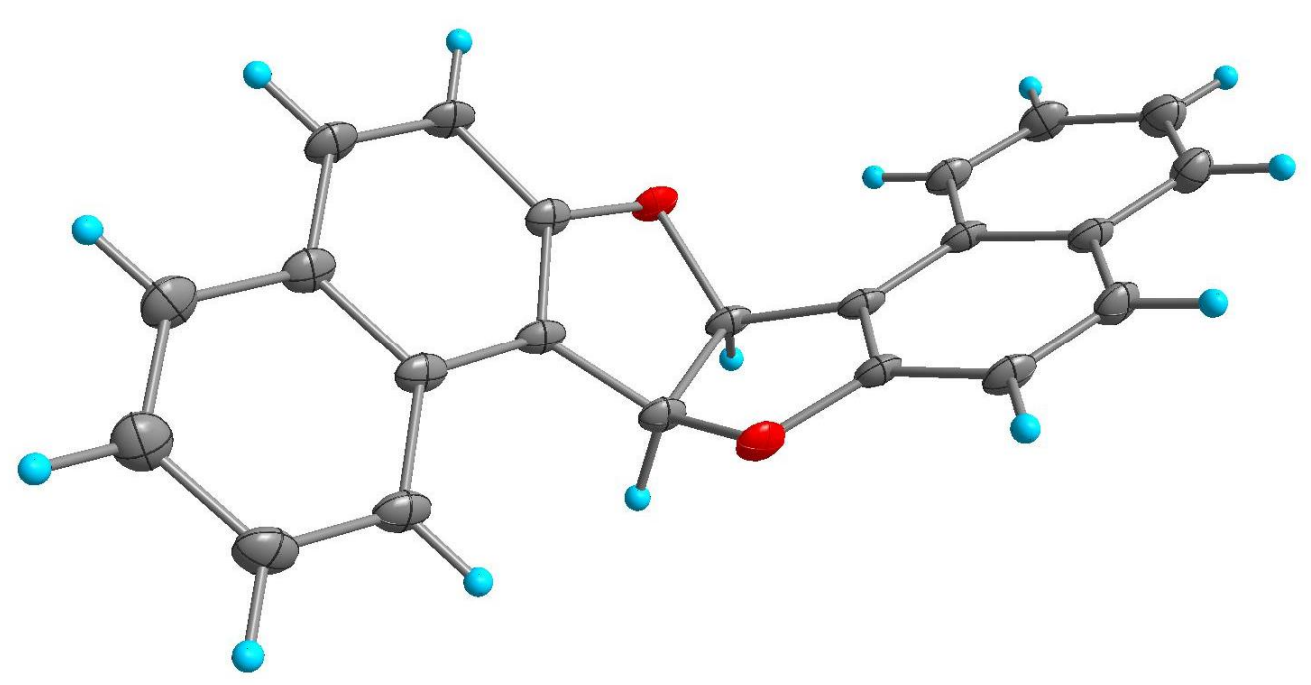

X-ray ORTEP drawing of compound $\mathbf{2 n}$ shown as 30\% probability. 


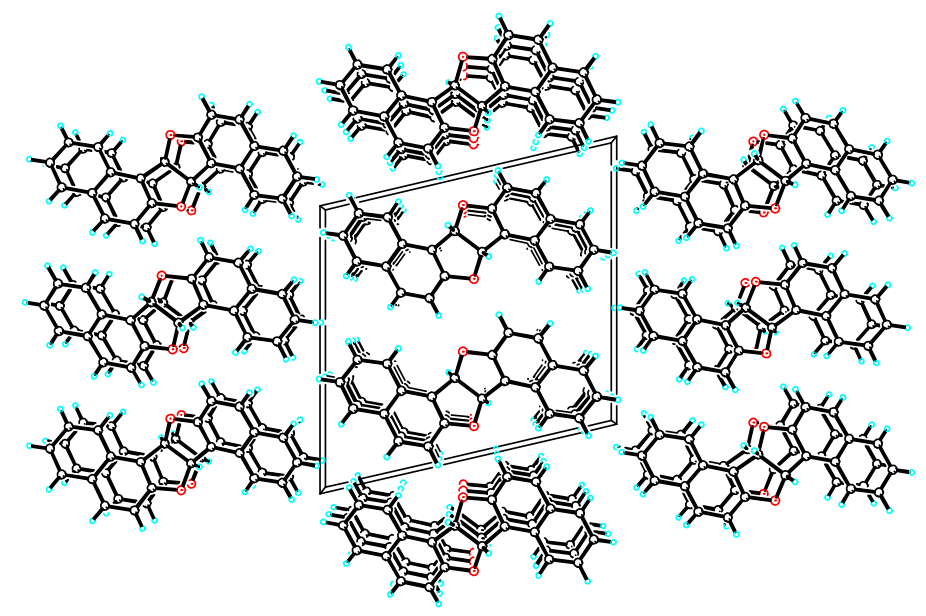

View of the pack drawing of $\mathbf{2 n}$.

Crystal data for $2 \mathrm{t}$ (CCDC 2038538): $\mathrm{C}_{17} \mathrm{H}_{15} \mathrm{ClO}_{2}, M=286.74, a=9.0232(5) \AA, b=$ 14.7894(7) $\AA, c=10.5810(5) \AA, \alpha=90^{\circ}, \beta=101.975(2)^{\circ}, \gamma=90^{\circ}, V=1381.28(12) \AA^{3}, T=$ 100.(2) K, space group $P 121 / n 1, Z=4, \mu(\mathrm{Cu} \mathrm{K \alpha})=2.429 \mathrm{~mm}^{-1}$, 14888 reflections measured, 2725 independent reflections $\left(R_{\text {int }}=0.0470\right)$. The final $R_{l}$ values were $0.0402(I>2 \sigma(I))$. The final $w R\left(F^{2}\right)$ values were $0.1040(I>2 \sigma(I))$. The final $R_{l}$ values were 0.0418 (all data). The final $w R\left(F^{2}\right)$ values were 0.1053 (all data). The goodness of fit on $F^{2}$ was 1.068 .

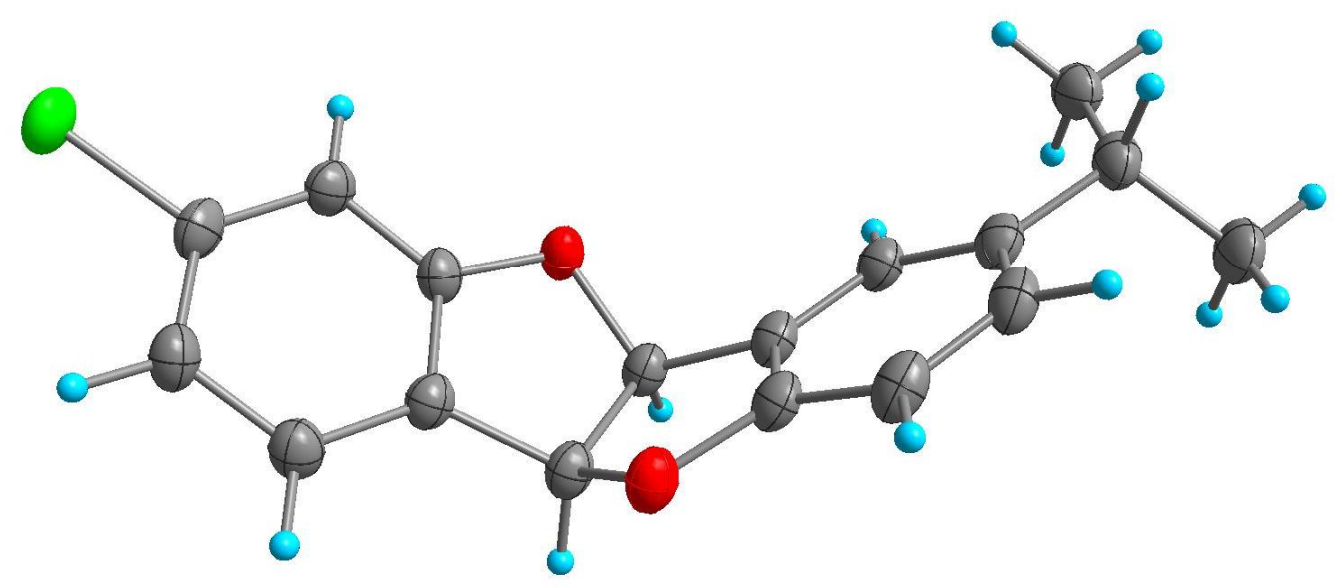

X-ray ORTEP drawing of compound $\mathbf{2 t}$ shown as $30 \%$ probability. 


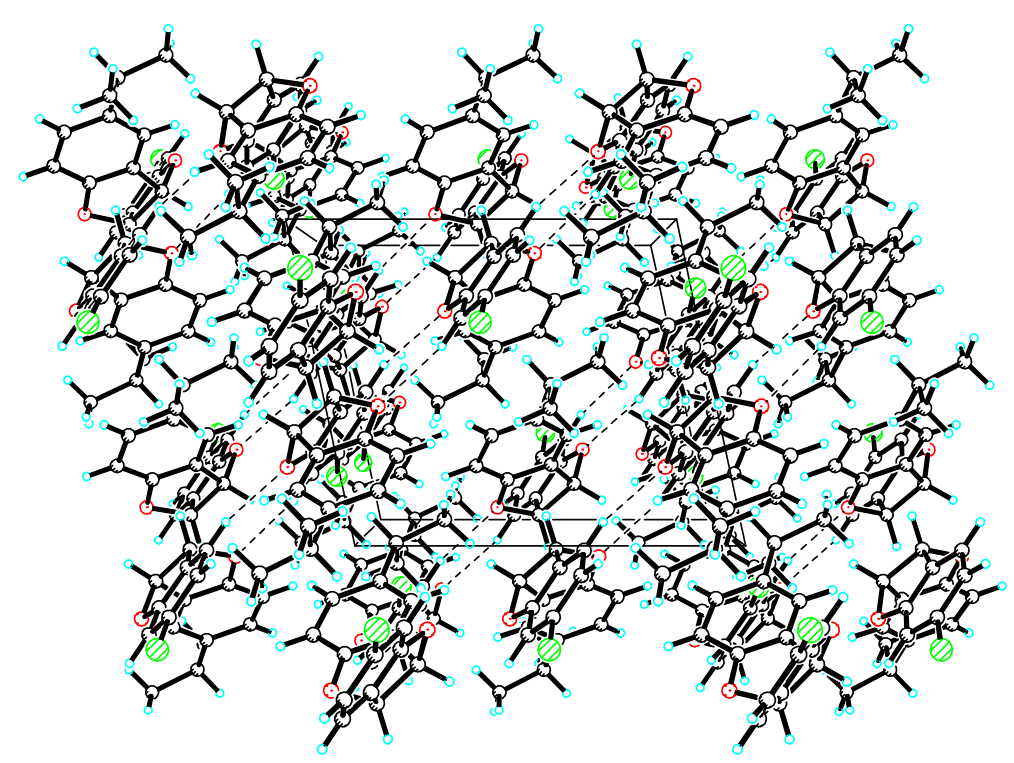

View of the pack drawing of $\mathbf{2 t}$.

Crystal data for 2am (CCDC 2038544): $\mathrm{C}_{16} \mathrm{H}_{14} \mathrm{O}_{2}, M=238.27, a=6.7179(3) \AA, b=9.7232(4) \AA$, $c=9.7237(4) \AA, \alpha=99.6530(10)^{\circ}, \beta=90.1450(10)^{\circ}, \gamma=104.2040(10)^{\circ}, V=606.38(4) \AA^{3}, T=$ 100.(2) $\mathrm{K}$, space group $P-1, Z=2, \mu(\mathrm{Cu} \mathrm{K \alpha})=0.678 \mathrm{~mm}^{-1}, 10280$ reflections measured, 2364 independent reflections $\left(R_{\text {int }}=0.0447\right)$. The final $R_{I}$ values were $0.0464(I>2 \sigma(I))$. The final $w R\left(F^{2}\right)$ values were $0.1256(I>2 \sigma(I))$. The final $R_{l}$ values were 0.0478 (all data). The final $w R\left(F^{2}\right)$ values were 0.1269 (all data). The goodness of fit on $F^{2}$ was 1.092 .

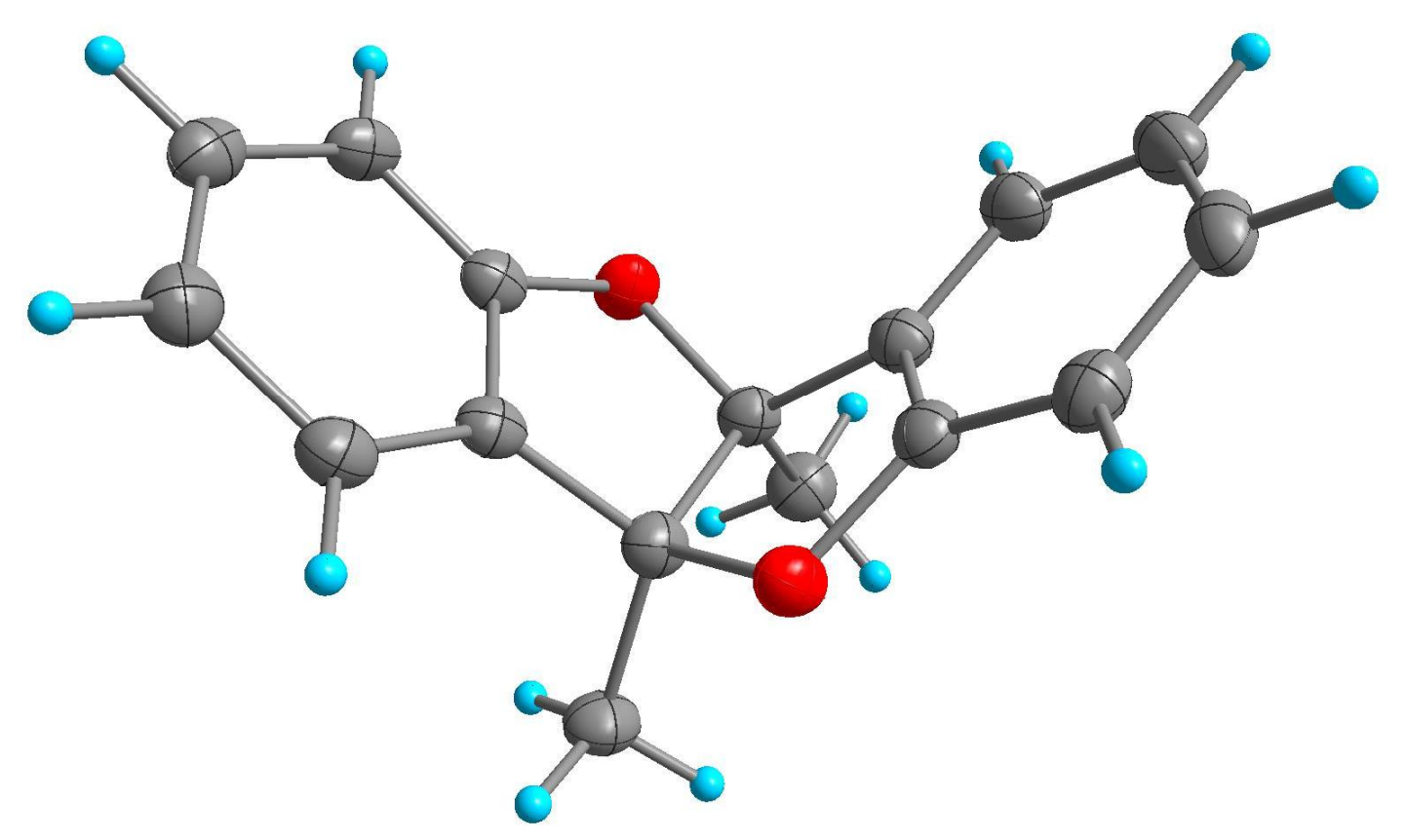

X-ray ORTEP drawing of compound 2am shown as 30\% probability. 


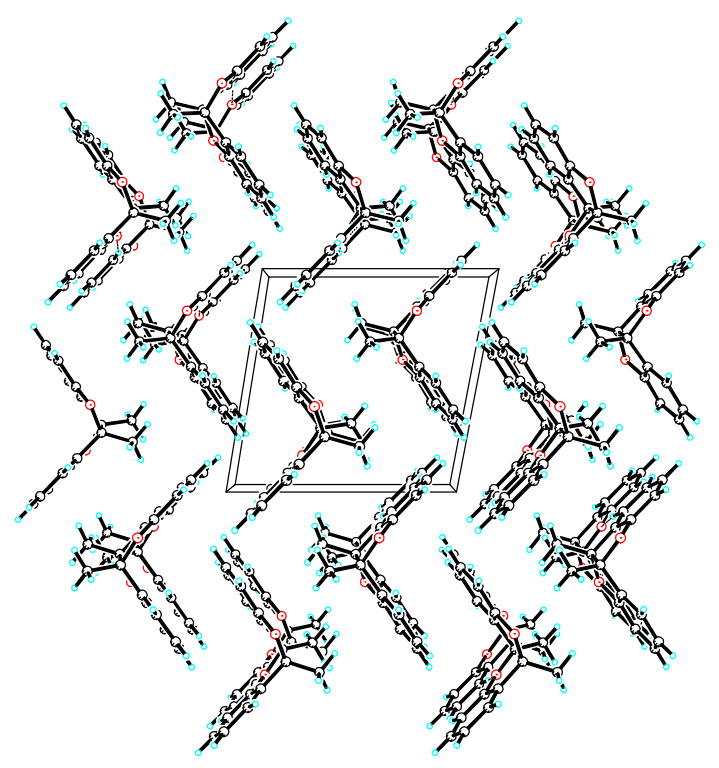

View of the pack drawing of $\mathbf{2 a m}$. 


\section{Details for determination of KIE values}

\section{Synthesis of 1a-D2}

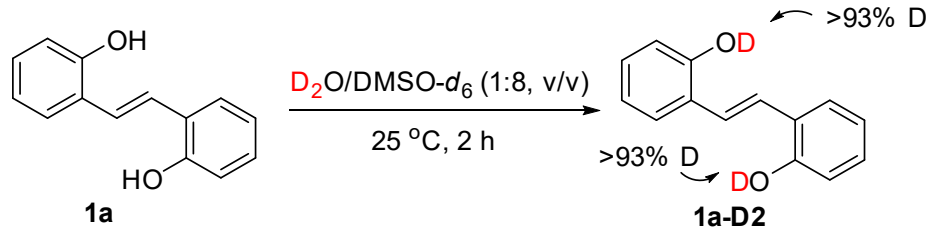

Scheme S2. Synthesis of deuterated 1a-D2.

${ }^{1} \mathrm{H}$ NMR spectrum of $\mathbf{1 a}$

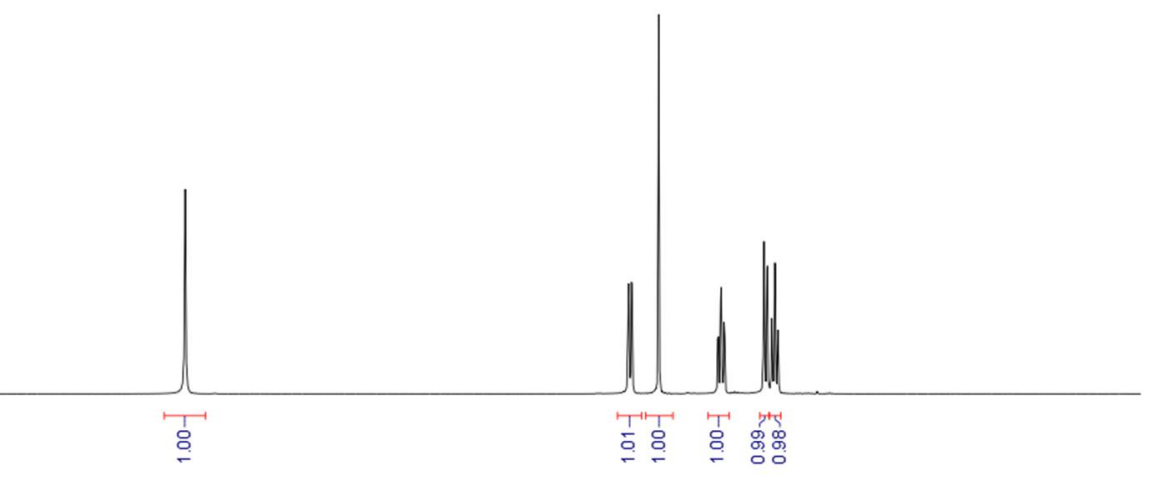

${ }^{1} \mathrm{H}$ NMR spectrum of 1a-D2

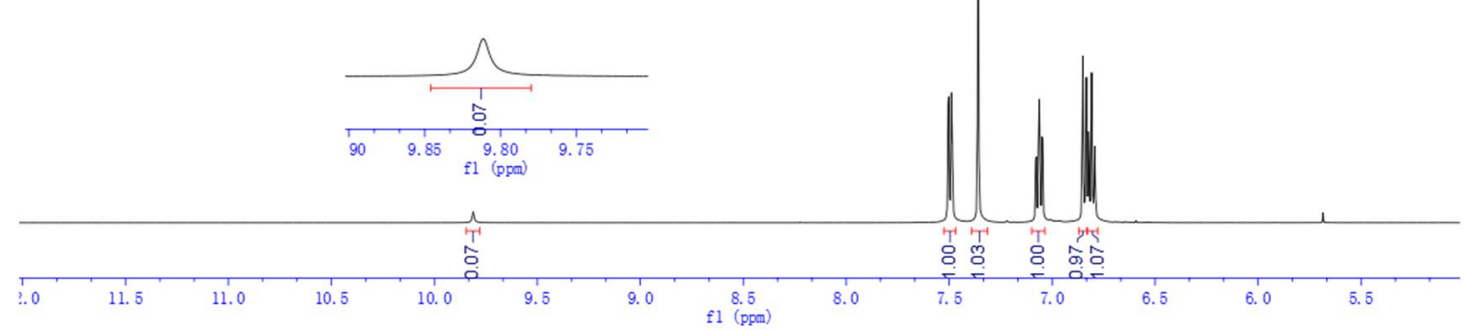

Figure S4. ${ }^{1} \mathrm{H}$ NMR spectra of 1a and 1a-D2.

\section{Synthesis of DBED- $d_{2}$}

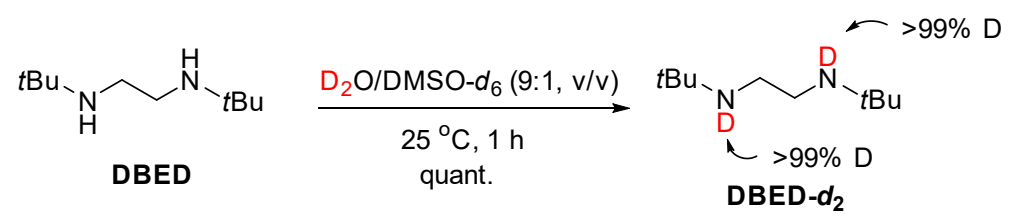

Scheme S3. Synthesis of deuterated DBED- $\boldsymbol{d}_{2}$. 
${ }^{1} \mathrm{H}$ NMR spectrum of DBED

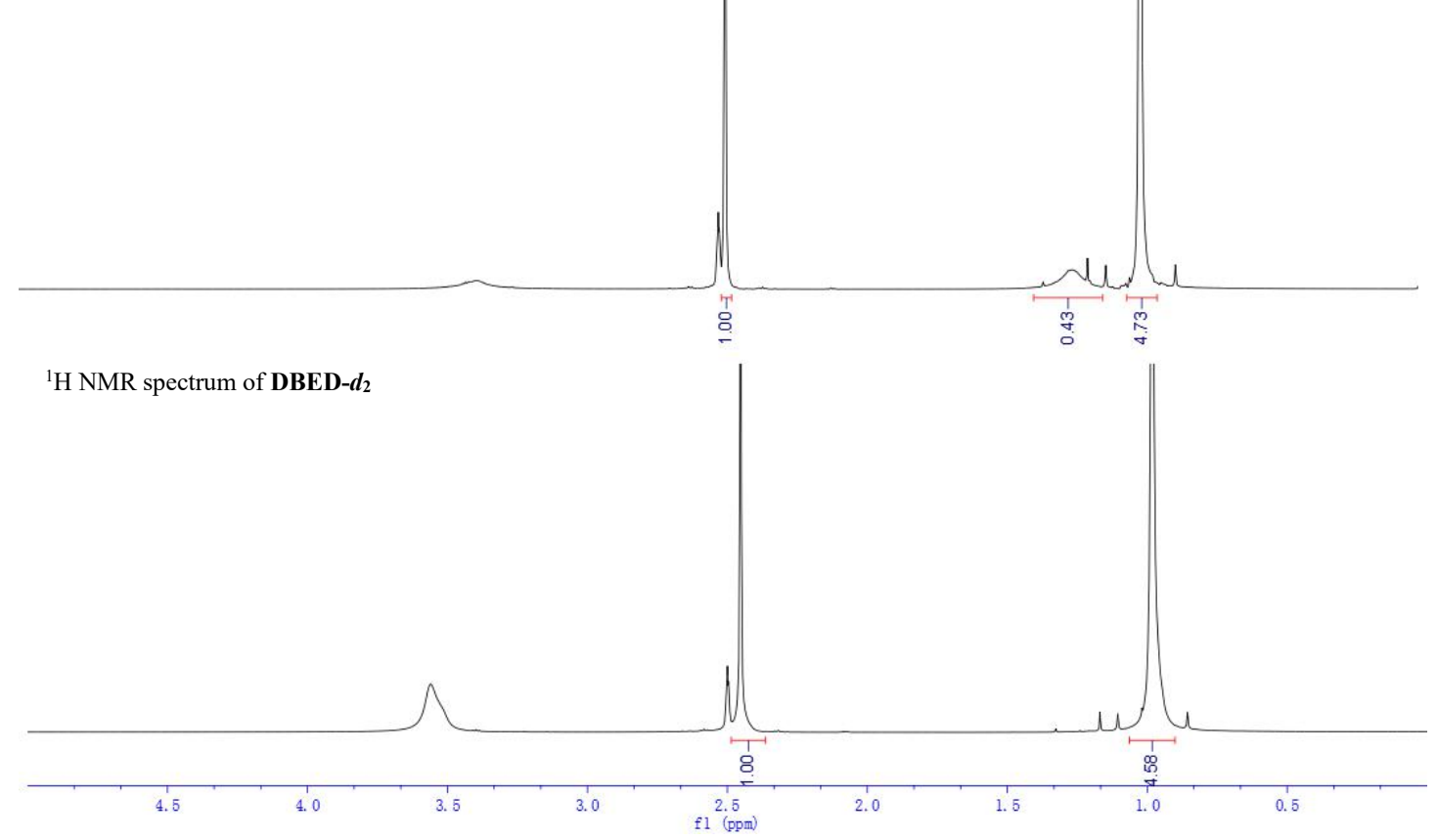

Figure S5. ${ }^{1} \mathrm{H}$ NMR spectra of DBED and DBED-d2.

\section{Determination of $k_{\mathrm{H}} / k_{\mathrm{D}}$}

The KIE value $\left(k_{\mathrm{H}} / k_{\mathrm{D}}\right)$ was determined by two separate, side-by-side reactions (serial 1 and 2). ${ }^{1,2}$ Based on the result obtained in Table 1 , entry $5, \mathrm{O}_{2}$ was utilized in place of air for avoiding the influence of incidental water. The $k_{\mathrm{H}} / k_{\mathrm{D}}$ value was calculated based on approximate equation (1), in which the $\mathrm{y}_{\mathrm{H}}$ and $\mathrm{y}_{\mathrm{D}}(\mathrm{mmol})$ was determined by ${ }^{1} \mathrm{H}$ NMR of crude products from serial 1 and 2 using hexamethylbenzene $(1.4 \mathrm{mg}$, $0.0086 \mathrm{mmol}$ ) as internal standard.

\section{Serial 1:}<smiles>Oc1ccccc1/C=C/c1ccccc1O</smiles>

1a $(0.05 \mathrm{mmol})$

\section{Serial 2:}<smiles>O=[N+]([O-])c1ccccc1/C=C/c1ccccc1[O+]</smiles>

1a-D2 (0.05 mmol)

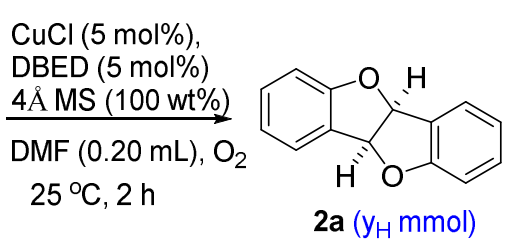

$\mathrm{CuCl}(5 \mathrm{~mol} \%)$ DBED- $d_{2}(5 \mathrm{~mol} \%)$ $4 \AA \mathrm{MS}(100 \mathrm{wt} \%)$ $\operatorname{DMF}(0.20 \mathrm{~mL}), \mathrm{O}_{2}$ $25^{\circ} \mathrm{C}, 2 \mathrm{~h}$

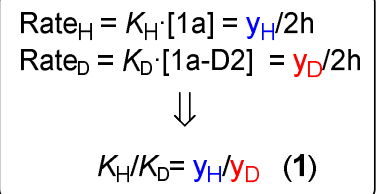




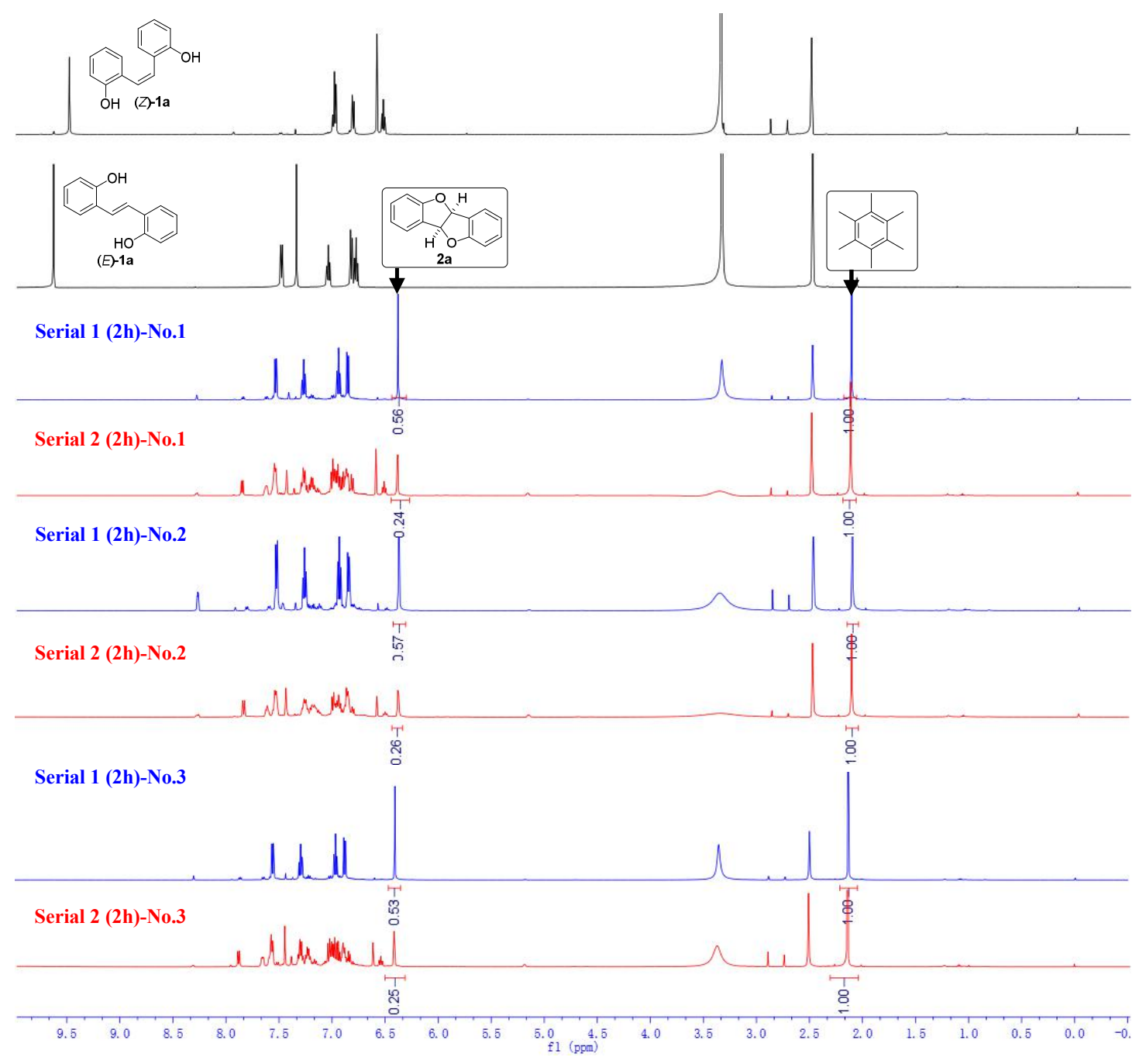

Figure S6. Crude NMR spectra (500 MHz in DMSO- $d_{6}$ ) of serials $\mathbf{1}$ and $\mathbf{2}$ at $2 \mathrm{~h}$.

\begin{tabular}{llll}
\hline No. & ун $(\mathbf{m m o l})$ & yD $(\mathrm{mmol})$ & KIE \\
\hline 1 & 0.0436 & 0.0187 & 2.33 \\
2 & 0.0443 & 0.0202 & 2.19 \\
3 & 0.0412 & 0.0194 & 2.12 \\
\hline
\end{tabular}

Based on above results, the KIE value was determined as $2.21 \pm 0.11$. 


\section{Computational details}

All DFT calculations were performed with Gaussian $16 .^{3}$ Geometry optimizations and frequency calculations were performed with SMD solvent model (n,n-dimethylformamide) using the combination of B3LYP(GD3BJ) functional and 6-311G(d) basis set. Single point energies were calculated with the B2PLYD3 functional and DEF2TZVP basis set in the gas phase. Solvation free energy were calculated using M062X/6-31G(d). ${ }^{4}, 5$ The 3D structures were prepared using CYLView. $^{6}$

Table S2. Calculated energies of optimized structures (a.u.)

\begin{tabular}{|c|c|c|c|c|}
\hline Structures & $\begin{array}{l}\text { Thermal correction to } \\
\text { gibbs free energy at } \\
\text { b3lyp }(g d 3 b j) / 6-311 g(d) \\
\text { (A) }\end{array}$ & $\begin{array}{l}\text { Single point energy in } \\
\text { gas at } \\
\text { b2plyp }(g d 3 b j) / d e f 2 t z v p \\
\text { (B) }\end{array}$ & $\begin{array}{l}\Delta G_{\text {sol-gas }} \text { at } \mathrm{M} 062 \mathrm{X} / 6-31 \mathrm{G}(\mathrm{d}) \\
\text { (C) }\end{array}$ & $G_{\text {sol }}{ }^{a}$ \\
\hline$(E)-\mathbf{1 a}$ & 0.181016 & -690.8523781 & $-690.8700988-(-690.8481517)$ & -690.6902973 \\
\hline$e z 1$ & 0.155034 & -689.5694689 & $-689.5953476-(-689.5733886)$ & -689.4333820 \\
\hline$(Z)-\mathbf{1 a}$ & 0.181353 & -690.8495263 & $-690.8690273-(-690.8459058)$ & -690.6882829 \\
\hline$z r 1$ & 0.156489 & -689.5613686 & $-689.5876757-(-689.5646162)$ & -689.4249272 \\
\hline$(Z, Z)-\boldsymbol{i}$ & 0.157919 & -689.5961758 & $-689.6127326-(-689.5912768)$ & -689.4542748 \\
\hline$p z z$ & 0.170848 & -689.9696145 & $-690.0469546-(-689.9631156)$ & -689.8795936 \\
\hline $2 \mathbf{a}$ & 0.166197 & -689.6563397 & $-689.6883813-(-689.6701779)$ & -689.5053342 \\
\hline $2 \mathbf{a}^{\prime}$ & 0.163136 & -689.669811 & $-689.6927763-(-689.6758615)$ & -689.5205779 \\
\hline
\end{tabular}

Cartesian coordinates of optimized structures.

(E)-1a

Three lowest frequencies $\left(\mathrm{cm}^{-1}\right): 19.1353,51.1915,57.8538$
C $\quad-3.7184397328 \quad-1.4517748585 \quad-0.8503761446$
$\begin{array}{llll}\text { C } & -4.6698915567 & -0.5911805717 & -0.297575889\end{array}$
$\begin{array}{llll}\text { C } & -4.2673762332 & 0.579222772 & 0.3336036976\end{array}$
$\begin{array}{llll}\text { C } & -2.912197239 & 0.9005541276 & 0.419529852\end{array}$
C $\quad \begin{array}{llll}\text { C } & .9327293288 & 0.0556553477 & -0.1461390138\end{array}$
$\begin{array}{llll}\text { C } & -2.3720342917 & -1.1220013368 & -0.7766156478\end{array}$ 

$\begin{array}{llll}\text { C } & -0.5174401932 & 0.429723544 & -0.0938319911\end{array}$
$\begin{array}{llll}\text { C } & 0.5174220819 & -0.4297292992 & -0.0938462809\end{array}$
$\begin{array}{llll}\text { C } & 1.932709708 & -0.0556667413 & -0.1461931793\end{array}$
$\begin{array}{llll}\text { C } & 2.3720040559 & 1.121993997 & -0.776660862\end{array}$
C $3.7184125772 \quad 1.4517594633 \quad-0.8504620472$
$\begin{array}{llll}\text { C } & 4.6698731822 & 0.5911493232 & -0.2977109714\end{array}$
$\begin{array}{llll}\text { C } & 4.2673679433 & -0.5792641689 & 0.3334639537\end{array}$
C $\quad 2.9121928929 \quad-0.9005858833 \quad 0.4194317777$
$\begin{array}{llll}\text { O } & -2.5992175544 & 2.0559317647 & 1.0734509396\end{array}$
$\begin{array}{llll}\text { O } & 2.5992202957 & -2.0559701197 & 1.0733437424\end{array}$
H $\quad-4.0274586506 \quad-2.3620654938 \quad-1.352186175$
$\begin{array}{llll}\mathrm{H} & -5.7260862717 & -0.8312601797 & -0.3576951482\end{array}$
$\begin{array}{llll}\mathrm{H} & -4.9896226981 & 1.2541868218 & 0.7795067879\end{array}$
$\begin{array}{llll}\mathrm{H} & -1.636579666 & -1.7690057107 & -1.2418947681\end{array}$
$\mathrm{H} \quad-0.2914809392 \quad 1.4948686743 \quad-0.0739803603$
$\begin{array}{llll}\mathrm{H} & 0.2914583353 & -1.4948731846 & -0.0739889125\end{array}$
H $\quad 1.6365432317 \quad 1.7690161099 \quad-1.2419060918$
$\begin{array}{llll}\mathrm{H} & 4.0274147701 & 2.362059355 & -1.352265428\end{array}$
$\begin{array}{llll}\mathrm{H} & 5.7260696118 & 0.8312144771 & -0.3578591132\end{array}$
$\begin{array}{llll}\mathrm{H} & 4.989629077 & -1.2542383167 & 0.7793280201\end{array}$
$\begin{array}{llll}\mathrm{H} & -1.6487229855 & 2.1020796851 & 1.2433467363\end{array}$
$\begin{array}{llll}\mathrm{H} & 1.648733628 & -2.1020982279 & 1.2432904366\end{array}$

ez1

Three lowest frequencies $\left(\mathrm{cm}^{-1}\right)$ : $-89.6257,43.9261,48.2531$

Imaginary frequency: -89.6257

$\begin{array}{llll}\text { C } & -4.1570661562 & 0.606969436 & -1.0068171224\end{array}$

C $\quad-4.739299953 \quad-0.4246712106 \quad-0.228666286$

$\begin{array}{llll}\text { C } & -3.9403406 & -1.2620721716 & 0.4969765555\end{array}$ 

$\begin{array}{llll}\text { C } & -2.490308324 & -1.1316091397 & 0.4996802673\end{array}$
$\begin{array}{llll}\text { C } & -1.905320911 & -0.0463498577 & -0.318199523\end{array}$
$\begin{array}{llll}\text { C } & -2.777546199 & 0.7776534545 & -1.0401574057\end{array}$
$\begin{array}{llll}\text { C } & -0.4834664668 & 0.214336217 & -0.4147475768\end{array}$
C $\quad 0.5279372803 \quad-0.4384976926 \quad 0.1954050405$
C $\quad 1.9518499346 \quad-0.0821708773 \quad 0.0072918573$
C $\quad 2.7492387872 \quad-0.7469334253 \quad-0.9059475885$

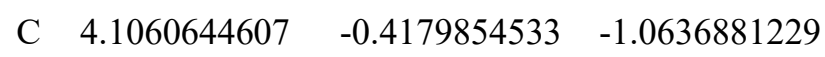
$\begin{array}{llll}\text { C } & 4.6992900731 & 0.5974037529 & -0.2870355537\end{array}$
$\begin{array}{llll}\text { C } & 3.946924712 & 1.2772390152 & 0.6371800094\end{array}$
$\begin{array}{llll}\text { C } & 2.5415373005 & 0.9749529334 & 0.8384027588\end{array}$

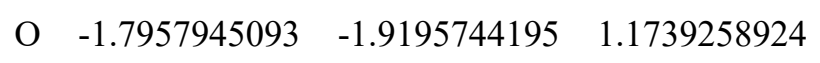
O $\quad 1.8637048776 \quad 1.5921405443 \quad 1.6914640349$
$\mathrm{H} \quad-4.79385736 \quad 1.2700180866 \quad-1.5821128538$
$\mathrm{H} \quad-5.8179394511 \quad-0.541941089 \quad-0.2148502772$
$\mathrm{H} \quad-4.3514765655 \quad-2.0622438299 \quad 1.1033028327$
$\mathrm{H} \quad-2.3585699572 \quad 1.5764367555 \quad-1.6444964734$
$\begin{array}{llll}\mathrm{H} & -0.226842284 & 1.0472394662 & -1.0673768744\end{array}$
$\mathrm{H} \quad 0.3071308071 \quad-1.269601898 \quad 0.8568110692$
$\begin{array}{llll}\mathrm{H} & 2.321996857 & -1.5351064647 & -1.517631895\end{array}$
H $\quad 4.701632177 \quad-0.9538227258 \quad-1.7946581327$
H $\quad 5.7488672289 \quad 0.8350743317 \quad-0.4251276053$
$\mathrm{H} \quad 4.3716118912 \quad 2.0631349918 \quad 1.2529569427$

\section{(Z)-1a}

Three lowest frequencies $\left(\mathrm{cm}^{-1}\right): 25.1700,26.9853,59.1247$
$\begin{array}{llll}\text { C } & 0.6632347697 & 2.0587049212 & 0.0107317359\end{array}$
C $\quad 2.7790847663 \quad 0.9331869336 \quad-0.6580052578$
$\begin{array}{llll}\text { C } & 1.5767408384 & 0.9076415023 & 0.0741569237\end{array}$ 

C $\quad 1.3331400424 \quad-0.2049772224 \quad 0.8914323828$
$\begin{array}{llll}\text { C } & 2.2297321967 & -1.2647537372 & 0.96878931\end{array}$
$\begin{array}{llll}\text { C } & -0.6807206472 & 2.0570815661 & 0.0201133492\end{array}$
$\begin{array}{llll}\text { C } & -1.5916812701 & 0.9059053269 & -0.0713818529\end{array}$
$\begin{array}{llll}\text { C } & -2.7944357088 & 0.9113269621 & 0.6605472529\end{array}$
C $\quad-3.6974103974 \quad-0.1494533756 \quad 0.5633080179$
$\begin{array}{llll}\text { C } & -3.4179254617 & -1.2272864808 & -0.2711582086\end{array}$
C $\quad-2.2396270941 \quad-1.245798988 \quad-1.0181950832$
C $\quad-1.3453357672 \quad-0.1862894509 \quad-0.9149797072$
$\begin{array}{llll}\text { C } & 3.4075832106 & -1.2258257823 & 0.221835948\end{array}$
C $\quad 3.6843476267 \quad-0.1276993256 \quad-0.58668444717$
H $\quad 1.1511447134 \quad 3.0290132545 \quad-0.0299302563$
H $\quad 2.0162749446 \quad-2.1107735105 \quad 1.6128315544$
H $\quad-1.1708022606 \quad 3.0250121001 \quad 0.0843881741$
H $\quad-4.6151834965 \quad-0.1282574054 \quad 1.1450996418$
H $\quad-4.1255488195 \quad-2.0466005343 \quad-0.3406601057$
H $\quad-2.0240559197 \quad-2.0756806074 \quad-1.6822208193$
H $\quad 4.1169763903 \quad-2.0450618679 \quad 0.2713314123$
H $\quad 4.6017473002 \quad-0.0906161866 \quad-1.1682719219$
H $\quad-0.4376544118-0.190230283 \quad-1.5065766154$
H $\quad 0.4257680595 \quad-0.2250387289 \quad 1.4831846539$
$\begin{array}{llll}\text { O } & -3.0270656994 & 1.9835057005 & 1.4711686382\end{array}$
H $\quad-3.8938391179 \quad 1.8896551456 \quad 1.8907734491$
$\begin{array}{llll}\text { O } & 3.0089772925 & 2.0249930442 & -1.4427956817\end{array}$
$\begin{array}{llll}\mathrm{H} & 3.8756925307 & 1.9430686396 & -1.8650113126\end{array}$

\section{$z r 1$}

Three lowest frequencies $\left(\mathrm{cm}^{-1}\right):-38.1940,54.3291,56.6619$

Imaginary frequency: -38.1940 

O $\quad 1.5041890117 \quad-0.2694183663 \quad 2.5091971103$
$\begin{array}{llll}\text { C } & 0.6538426995 & 1.7207664597 & 0.660095369\end{array}$
$\begin{array}{llll}\text { C } & 2.38287048 & 0.7355383572 & -0.8428518697\end{array}$
$\begin{array}{llll}\text { C } & 1.6784543062 & 0.7041751715 & 0.3494727386\end{array}$
C $\quad 2.0523255383 \quad-0.2669944647 \quad 1.3860386515$
$\begin{array}{llll}\text { C } & 3.1064138814 & -1.2100577542 & 1.0587464991\end{array}$
$\begin{array}{llll}\text { C } & -0.6946745958 & 1.7126787751 & 0.5593198194\end{array}$
$\begin{array}{llll}\text { C } & -1.6734226616 & 0.7344718456 & 0.120739518\end{array}$
$\begin{array}{llll}\text { C } & -3.0167446102 & 1.1354906793 & 0.1247142576\end{array}$
$\begin{array}{llll}\text { C } & -4.0472966586 & 0.2961890153 & -0.2838703663\end{array}$
$\begin{array}{llll}\text { C } & -3.7727080475 & -1.020000057 & -0.7282471779\end{array}$
$\begin{array}{llll}\text { C } & -2.4832550394 & -1.471417565 & -0.7505380898\end{array}$
C $\quad-1.3688701319 \quad-0.6379926086 \quad-0.3252663859$
C $\quad 3.7550875209-1.1664132991 \quad-0.1478074991$
C $3.402531539-0.1874895683-1.1031534525$
$\begin{array}{llll}\text { O } & -0.2093608443 & -1.098329486 & -0.3450166398\end{array}$
H $\quad 1.0755939768 \quad 2.6550601473 \quad 1.0323350797$
H $\quad 2.1304688892 \quad 1.4754445996 \quad-1.5956889913$
H $\quad 3.3647832394 \quad-1.9388653387 \quad 1.8201419334$
$\begin{array}{llll}\mathrm{H} & -1.1627676227 & 2.6478579218 & 0.8594589356\end{array}$
H $\quad-3.2587721237 \quad 2.1397230076 \quad 0.4582282029$
$\begin{array}{llll}\mathrm{H} & -5.0705049357 & 0.6547385001 & -0.2633601215\end{array}$
H $\quad-4.5882098933 \quad-1.6614433489 \quad-1.0458861303$
H $\quad-2.2338453476 \quad-2.4743687149 \quad-1.0802327817$
H $\quad 4.5471422778 \quad-1.8734251458 \quad-0.373005505$
H $\quad 3.9281528119-0.1495758425 \quad-2.0512919644$

\section{$(Z, Z)-i$}

Three lowest frequencies $\left(\mathrm{cm}^{-1}\right): 27.9946,38.4944,61.0292$ 

C $\quad 4.0572976054 \quad 1.376101272 \quad-0.2034724107$
$\begin{array}{llll}\text { C } & 4.6811370387 & 0.0897925191 & -0.0182466791\end{array}$
$\begin{array}{llll}\text { C } & 3.9486611583 & -1.0288804584 & 0.1986771791\end{array}$
C $\quad 2.4932662393 \quad-0.9807712152 \quad 0.300589638$
$\begin{array}{llll}\text { C } & 1.8535068529 & 0.344612659 & 0.0100774244\end{array}$
$\begin{array}{llll}\text { C } & 2.7062189988 & 1.4907549284 & -0.1857423475\end{array}$
C $\quad 0.4912741113 \quad 0.5138161296 \quad-0.1006142467$
C $\quad-0.4912615451 \quad-0.5137119361 \quad-0.100514341$
$\begin{array}{llll}\text { O } & 1.8427009227 & -1.9767761032 & 0.6297860254\end{array}$
H $\quad-0.1358857009 \quad-1.5284713475 \quad-0.2361458775$
$\begin{array}{llll}\text { C } & -1.8534998663 & -0.3445561028 & 0.0102544302\end{array}$
$\begin{array}{llll}\text { C } & -2.4933459206 & 0.98080919 & 0.3007187878\end{array}$
$\begin{array}{llll}\text { C } & -3.9487265721 & 1.028876892 & 0.1985987291\end{array}$
C $\quad-4.6811374222 \quad-0.0898503592 \quad-0.0182633012$
$\begin{array}{llll}\text { C } & -4.0572345448 & -1.376157309 & -0.2032777714\end{array}$

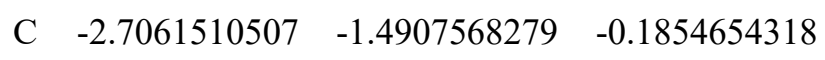
$\mathrm{H} \quad 0.1359231144 \quad 1.5285720904 \quad-0.2363230452$
$\begin{array}{llll}\text { O } & -1.8428731082 & 1.9768378534 & 0.6300217509\end{array}$
$\begin{array}{llll}\mathrm{H} & 4.6825848895 & 2.2455782457 & -0.3704610837\end{array}$
$\begin{array}{llll}\text { H } & 5.7645121639 & 0.029886202 & -0.0494215627\end{array}$
H $\quad 4.413450272 \quad-1.9955210146 \quad 0.3588292041$
$\begin{array}{llll}\mathrm{H} & 2.2302184312 & 2.4531106536 & -0.3445957184\end{array}$
$\mathrm{H} \quad-4.4135712221 \quad 1.9955117975 \quad 0.3586205656$
$\mathrm{H} \quad-5.7645124522 \quad-0.0299942404 \quad-0.0495330987$
H $\quad-4.6824810156 \quad-2.2456760507 \quad-0.370201892$
H $\quad-2.2301015276 \quad-2.4531074877 \quad-0.3442016271$

Pzz.

Three lowest frequencies $\left(\mathrm{cm}^{-1}\right): 25.5569,38.8079,58.5676$ 

C $\quad 4.13377155 \quad 1.22639235 \quad-0.36557245$
$\begin{array}{llll}\text { C } & 4.68559381 & -0.1007246 & -0.20544368\end{array}$
$\begin{array}{llll}\text { C } & 3.89674732 & -1.15872554 & 0.08017883\end{array}$
$\begin{array}{llll}\text { C } & 2.4639263 & -0.97220491 & 0.31048012\end{array}$
$\begin{array}{llll}\text { C } & 1.88749509 & 0.38441267 & -0.0328419\end{array}$
$\begin{array}{llll}\text { C } & 2.79667683 & 1.45970716 & -0.2700785\end{array}$
$\begin{array}{llll}\text { C } & 0.52668587 & 0.59735595 & -0.14394643\end{array}$
C $\quad-0.44240832 \quad-0.42303813 \quad-0.17819943$
$\begin{array}{llll}\text { O } & 1.77401678 & -1.85116297 & 0.81415543\end{array}$
H $\quad-0.08352004 \quad-1.43452576 \quad-0.32250163$
$\begin{array}{llll}\text { C } & -1.83714658 & -0.2961496 & -0.1137452\end{array}$
$\begin{array}{llll}\text { C } & -2.54410166 & 0.91984068 & 0.18747016\end{array}$
$\begin{array}{llll}\text { C } & -3.93786581 & 0.94073017 & 0.16856328\end{array}$
$\begin{array}{llll}\text { C } & -4.64315008 & -0.21807588 & -0.11032896\end{array}$

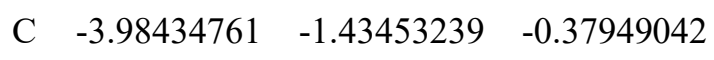
$\begin{array}{llll}\text { C } & -2.61542677 & -1.46848317 & -0.37417414\end{array}$
$\begin{array}{llll}\text { H } & 0.20500687 & 1.62239624 & -0.27386792\end{array}$
$\begin{array}{llll}\text { O } & -1.82984927 & 2.00540818 & 0.49626219\end{array}$
$\mathrm{H} \quad 4.80931107 \quad 2.04198654 \quad-0.59550243$
H $\quad 5.75742741 \quad-0.22836308 \quad-0.30836121$
H $\quad 4.2932551 \quad-2.15368564 \quad 0.24360218$
H $\quad 2.39323654 \quad 2.45246417 \quad-0.43418158$
$\begin{array}{llll}\mathrm{H} & -4.46265669 & 1.86532617 & 0.3866625\end{array}$
H $\quad-5.72674545 \quad-0.18413697 \quad-0.1131708$
H $\quad-4.55933522 \quad-2.32775969 \quad-0.58875088$
H $\quad-2.08478699 \quad-2.39002792 \quad-0.58345241$
H $\quad-2.41323232 \quad 2.76333576 \quad 0.66895566$

\section{2a}

Three lowest frequencies $\left(\mathrm{cm}^{-1}\right): 32.5844,85.6617,132.1847$ 

C $\quad 3.7631478216 \quad-0.4170475502 \quad 1.413886783$
$\begin{array}{llll}\text { C } & 3.7471152697 & 0.8203103354 & 0.7623349261\end{array}$
$\begin{array}{llll}\text { C } & 2.6648441861 & 1.1720559709 & -0.0472223514\end{array}$
$\begin{array}{llll}\text { C } & 1.61622776 & 0.2731859655 & -0.1892229882\end{array}$
C $\quad 1.6466089616 \quad-0.9588793919 \quad 0.464288769$
C $\quad 2.7142090292 \quad-1.3279267667 \quad 1.2757698202$
$\begin{array}{llll}\text { C } & 0.3264276647 & 0.3795913903 & -0.9402771819\end{array}$
C $\quad-0.3311694966 \quad-1.0064381949 \quad-0.6840879192$
$\begin{array}{llll}\text { O } & 0.5630141731 & -1.7423271952 & 0.2191179593\end{array}$
$\begin{array}{llll}\text { O } & -0.5664205313 & 1.3895271741 & -0.3574136558\end{array}$
$\begin{array}{llll}\text { C } & -1.6493365248 & 0.7454100059 & 0.1529616697\end{array}$
$\begin{array}{llll}\text { C } & -1.6196962723 & -0.638724435 & -0.0181494502\end{array}$
C $\quad-2.7156427641 \quad 1.3799721104 \quad 0.7812839677$
$\begin{array}{llll}\text { C } & -3.7640479388 & 0.5786094947 & 1.2370408977\end{array}$
C $\quad \begin{array}{llll}-3.7487511252 & -0.809765762 & 1.069673624\end{array}$
C $\quad-2.6677760991 \quad-1.4274826793 \quad 0.4369516502$
H $\quad-0.4358252666 \quad-1.6195808812 \quad-1.5776747608$
$\begin{array}{llll}\mathrm{H} & 0.4293335879 & 0.6336355954 & -1.9939688583\end{array}$
H $\quad 4.6090824211 \quad-0.6804171481 \quad 2.0402210851$
H $\quad 4.5768050849 \quad 1.5069548137 \quad 0.8876079971$
H $\quad 2.6402264686 \quad 2.1313621933 \quad-0.5535883034$
H $\quad 2.7261463543 \quad-2.2877249852 \quad 1.7790958103$
H $\quad-2.7270130966 \quad 2.4561727208 \quad 0.9091711373$
H $\quad-4.6089769838 \quad 1.04833339526 \quad 1.7297044667$
H $\quad-4.5780019003 \quad-1.4062947323 \quad 1.4330680958$
$\begin{array}{llll}\mathrm{H} & -2.6437307829 & -2.504312001 & 0.3060268098\end{array}$

\section{$2 \mathbf{a}^{\prime}$}

Three lowest frequencies ( $\left.\mathrm{cm}^{-1}\right): 20.9581,59.4954,121.5919$ 

C $\quad-3.8152057922 \quad-0.1953890679 \quad 1.7935413481$
C $\quad-3.2076211269 \quad-1.0439531982 \quad 2.7350112144$
C $\quad-1.8513998886 \quad-1.3445562255 \quad 2.6725099896$
$\begin{array}{llll}\text { C } & -1.0910841829 & -0.7780822199 & 1.6394987347\end{array}$
$\begin{array}{llll}\text { C } & -1.7328341023 & 0.0650399956 & 0.7173556848\end{array}$
$\begin{array}{llll}\text { C } & -3.0799682259 & 0.3795599053 & 0.7573190413\end{array}$
$\begin{array}{llll}\text { C } & 0.2874629647 & -0.834565485 & 1.2336809671\end{array}$
$\begin{array}{llll}\text { C } & 0.4157287856 & -0.0498921091 & 0.1257513082\end{array}$
$\begin{array}{llll}\text { O } & -0.8183891143 & 0.5115009159 & -0.2046022148\end{array}$
$\begin{array}{llll}\text { C } & 1.545608112 & 0.2884314175 & -0.7272150878\end{array}$
C $\quad 2.8264410772 \quad-0.1783831915 \quad-0.3694758089$
C $\quad 3.9437826602 \quad 0.0916295767 \quad-1.1402742111$
$\begin{array}{llll}\text { C } & 3.8071102711 & 0.8502060943 & -2.307084268\end{array}$
$\begin{array}{llll}\text { C } & 2.5609181299 & 1.3260315248 & -2.6824877654\end{array}$
C $\quad 1.4256719186 \quad 1.0575642614 \quad-1.9099473095$
$\begin{array}{llll}\text { O } & 0.2578736563 & 1.5698689178 & -2.3734382784\end{array}$
H $\quad-4.8755044393 \quad 0.0173281311 \quad 1.8721271006$
H $\quad-3.8131702852 \quad-1.4721340049 \quad 3.5265427394$
Н $\quad-1.3919514364 \quad-2.0003141159 \quad 3.4038345159$
$\begin{array}{llll}\mathrm{H} & -3.5351995258 & 1.035492928 & 0.0245350701\end{array}$
H $\quad 1.0756502633 \quad-1.3963214909 \quad 1.7103000073$
H $\quad 2.9357079488 \quad-0.7617466799 \quad 0.5372390769$
H $\quad 4.9150091918 \quad-0.2817780339 \quad-0.8364272324$
H $\quad 4.6730603041 \quad 1.0715382154 \quad-2.9216256255$
H $\quad 2.4335927231 \quad 1.9169567211 \quad-3.5826577791$
$\begin{array}{llll}\mathrm{H} & -0.465989887 & 1.3510672175 & -1.7686112173\end{array}$ 


\section{Reference}

1. García-Cuadrado, D.; Braga, A. A. C.; Maseras, F.; Echavarren, A. M., Proton Abstraction Mechanism for the Palladium-Catalyzed Intramolecular Arylation. J. Am. Chem. Soc. 2006, 128, 1066-1067.

2. Simmons, E. M.; Hartwig, J. F., On the Interpretation of Deuterium Kinetic Isotope Effects in C H Bond Functionalizations by Transition-Metal Complexes. Angew. Chem. Int. Ed. 2012, 51, 3066-3072.

3. M. J. Frisch, G. W. Trucks, H. B. Schlegel, G. E. Scuseria, M. A. Robb, J. R. Cheeseman, G. Scalmani, V. Barone, G. A. Petersson, H. Nakatsuji, X. Li, M. Caricato, A. V. Marenich, J. Bloino, B. G. Janesko, R. Gomperts, B. Mennucci, H. P. Hratchian, J. V. Ortiz, A. F. Izmaylov, J. L. Sonnenberg, D. Williams-Young, F. Ding, F. Lipparini, F. Egidi, J. Goings, B. Peng, A. Petrone, T. Henderson, D. Ranasinghe, V. G. Zakrzewski, J. Gao, N. Rega, G. Zheng, W. Liang, M. Hada, M. Ehara, K. Toyota, R. Fukuda, J. Hasegawa, M. Ishida, T. Nakajima, Y. Honda, O. Kitao, H. Nakai, T. Vreven, K. Throssell, J. A. Montgomery, Jr., J. E. Peralta, F. Ogliaro, M. J. Bearpark, J. J. Heyd, E. N. Brothers, K. N. Kudin, V. N. Staroverov, T. A. Keith, R. Kobayashi, J. Normand, K. Raghavachari, A. P. Rendell, J. C. Burant, S. S. Iyengar, J. Tomasi, M. Cossi, J. M. Millam, M. Klene, C. Adamo, R. Cammi, J. W. Ochterski, R. L. Martin, K. Morokuma, O. Farkas, J. B. Foresman, and D. J. Fox, Gaussian, Inc., Wallingford CT, 2016.

4. Ho, J.; Klamt, A.; Coote, M. L., Comment on the Correct Use of Continuum Solvent Models. J. Phys. Chem. A 2010, 114, 13442-13444.

5. Ribeiro, R. F.; Marenich, A. V.; Cramer, C. J.; Truhlar, D. G., Use of Solution-Phase Vibrational Frequencies in Continuum Models for the Free Energy of Solvation. J. Phys. Chem. B 2011, 115, 14556-14562.

6. CYLview, v1.0 beta; Legault, C. Y., Université de Sherbrooke, 2009 (http://www.cylview.org). 
NMR spectra copies for all synthetic compounds.

NMR spectra of 1a-1an

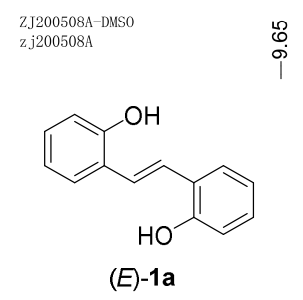

${ }^{1} \mathrm{H}$ NMR (500 MHz, DMSO- $d_{6}$ )
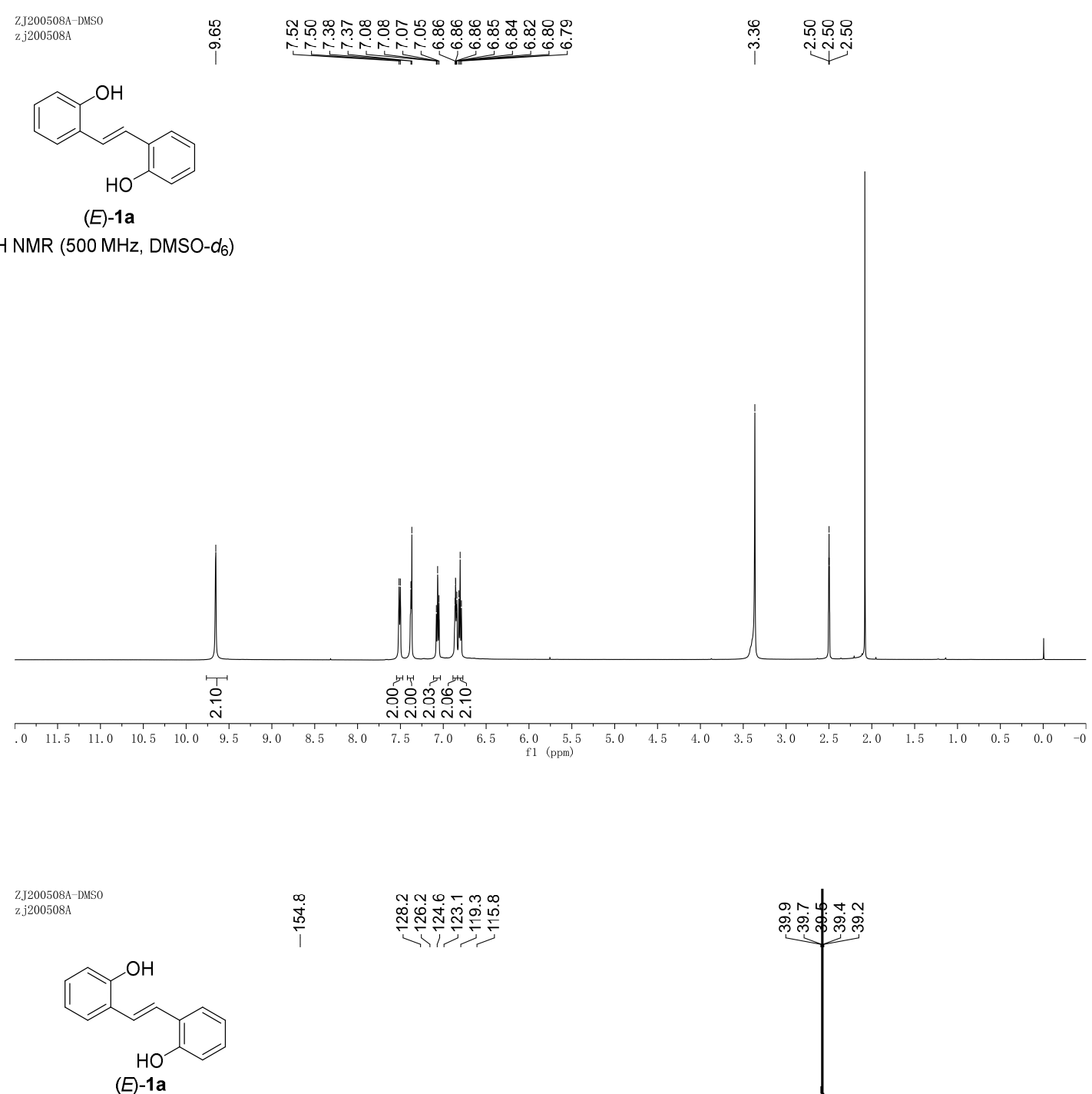

${ }^{13} \mathrm{C}$ NMR (125 MHz, DMSO-d $\left.d_{6}\right)$
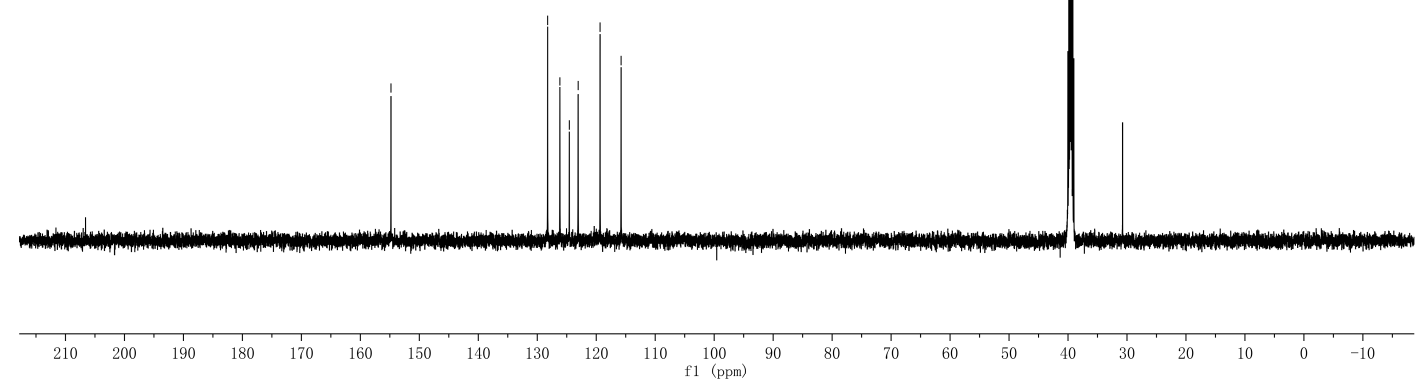


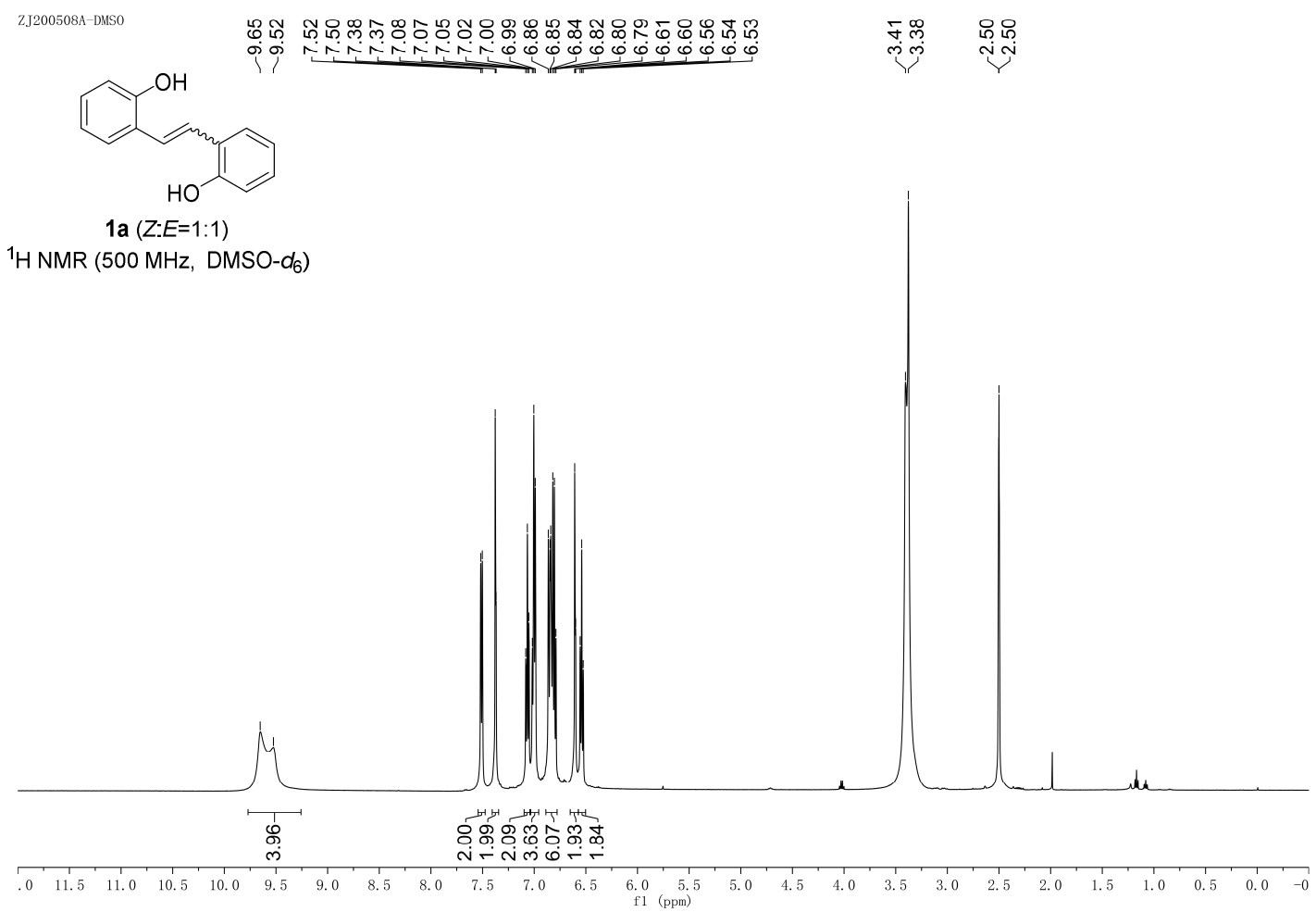

ZJ200508A-DMSO

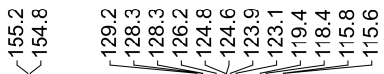<smiles>Oc1ccccc1/C=C/c1ccccc1O</smiles>

1a $(Z: E=1: 1)$

${ }^{13} \mathrm{H} \mathrm{NMR}\left(125 \mathrm{MHz}\right.$, Methanol- $\left.d_{4}\right)$

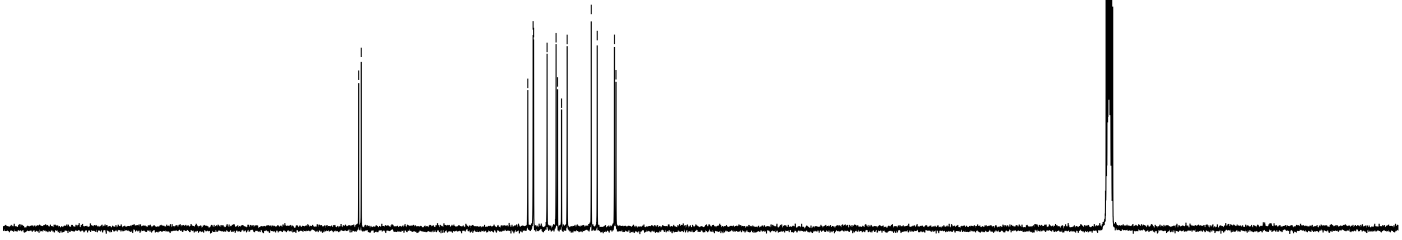

$\begin{array}{llllllllllll}10 & 200 & 190 & 180 & 170 & 160 & 150 & 140 & 130 & 120 & 110 & 100\end{array}$ 


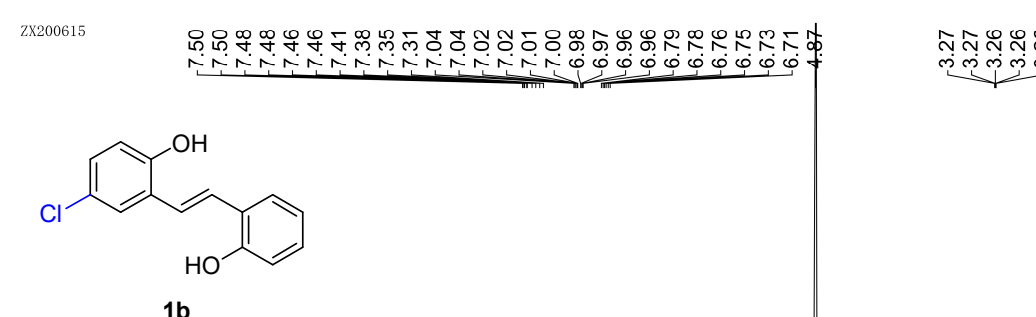

${ }^{1} \mathrm{H}$ NMR $\left(500 \mathrm{MHz}\right.$, Methanol- $\left.d_{4}\right)$

Zx200615
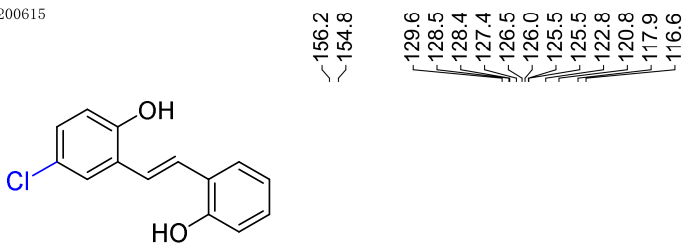

1b

${ }^{13} \mathrm{C}$ NMR $\left(125 \mathrm{MHz}\right.$, Methanol- $\left.d_{4}\right)$

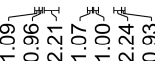

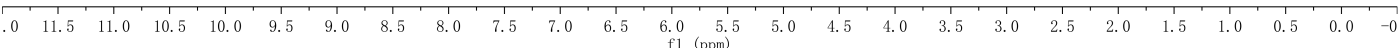

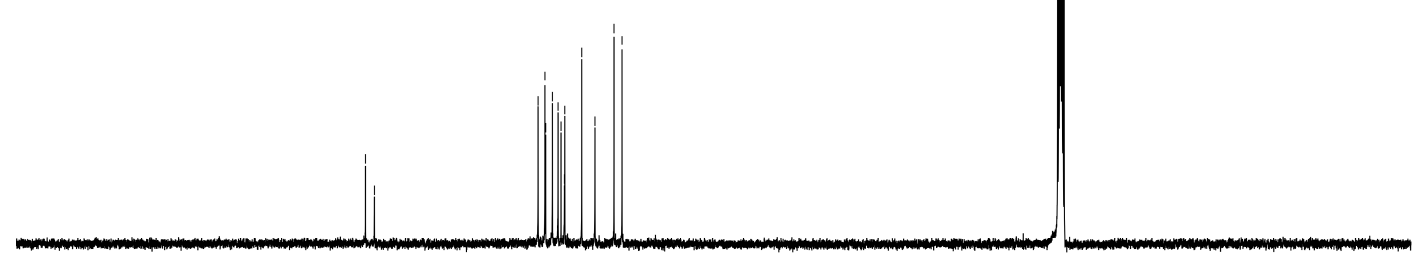

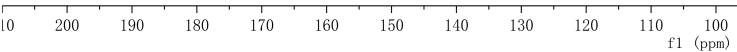




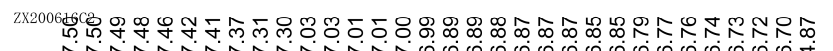

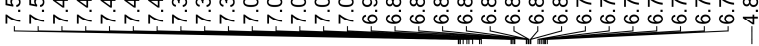

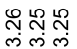

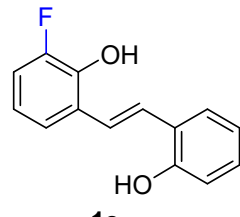

$1 \mathrm{c}$

${ }^{1} \mathrm{H}$ NMR $\left(500 \mathrm{MHz}\right.$, Methanol- $\left.d_{4}\right)$

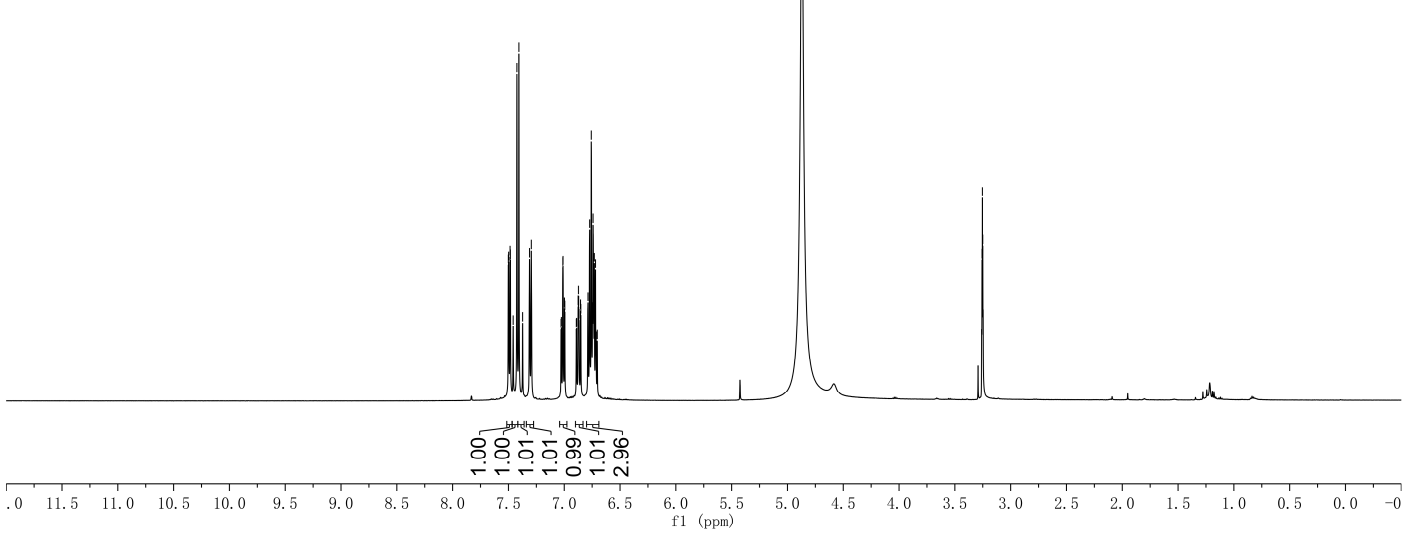

ZX200616C2

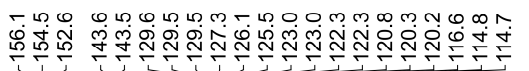

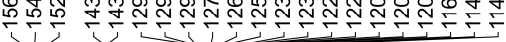

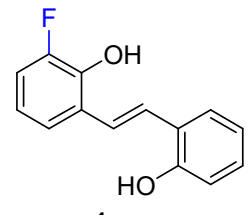

${ }^{13} \mathrm{C}$ NMR $\left(125 \mathrm{MHz}\right.$, Methanol- $\left.d_{4}\right)$

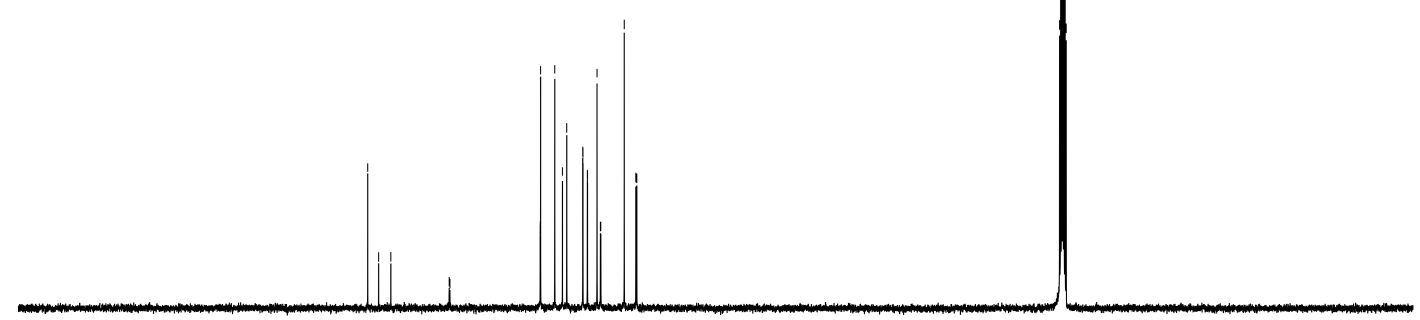

$\begin{array}{lllllllllllll}10 & 200 & 190 & 180 & 170 & 160 & 150 & 140 & 130 & 120 & 110 & 1 \\ \mathrm{f} 1(\mathrm{pmm})\end{array}$ 
zx200616C2F<smiles>Oc1ccccc1/C=C/c1cccc(F)c1O</smiles>

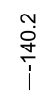

${ }^{19} \mathrm{~F}$ NMR $\left(471 \mathrm{MHz}\right.$, Methanol- $\left.d_{4}\right)$

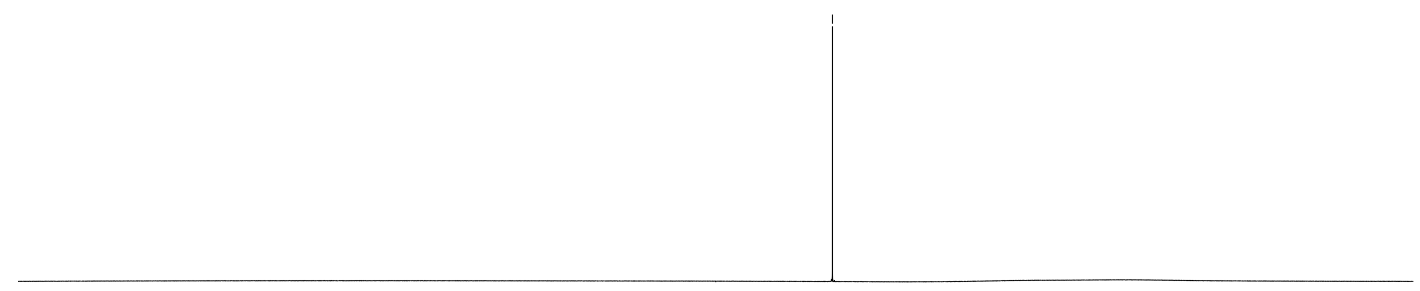

\begin{tabular}{lllllllllllllllllllllllllllllll}
\hline & 5 & -10 & -20 & -30 & -40 & -50 & -60 & -70 & -80 & -90 & -100 & -110 & -120 & -130 & -140 & -150 & -160 & -170 & -180 & -190 & -200 & -210 & -220 & -230 & -2 \\
\hline
\end{tabular} 
ZX200611A1

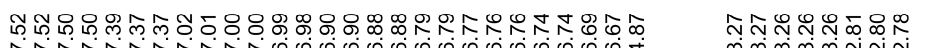

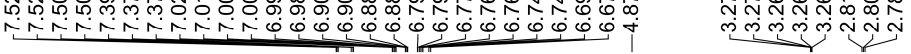

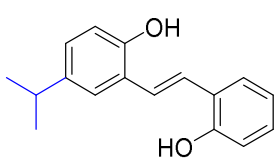

$1 d$

${ }^{1} \mathrm{H}$ NMR $\left(500 \mathrm{MHz}\right.$, Methanol- $\left.d_{4}\right)$

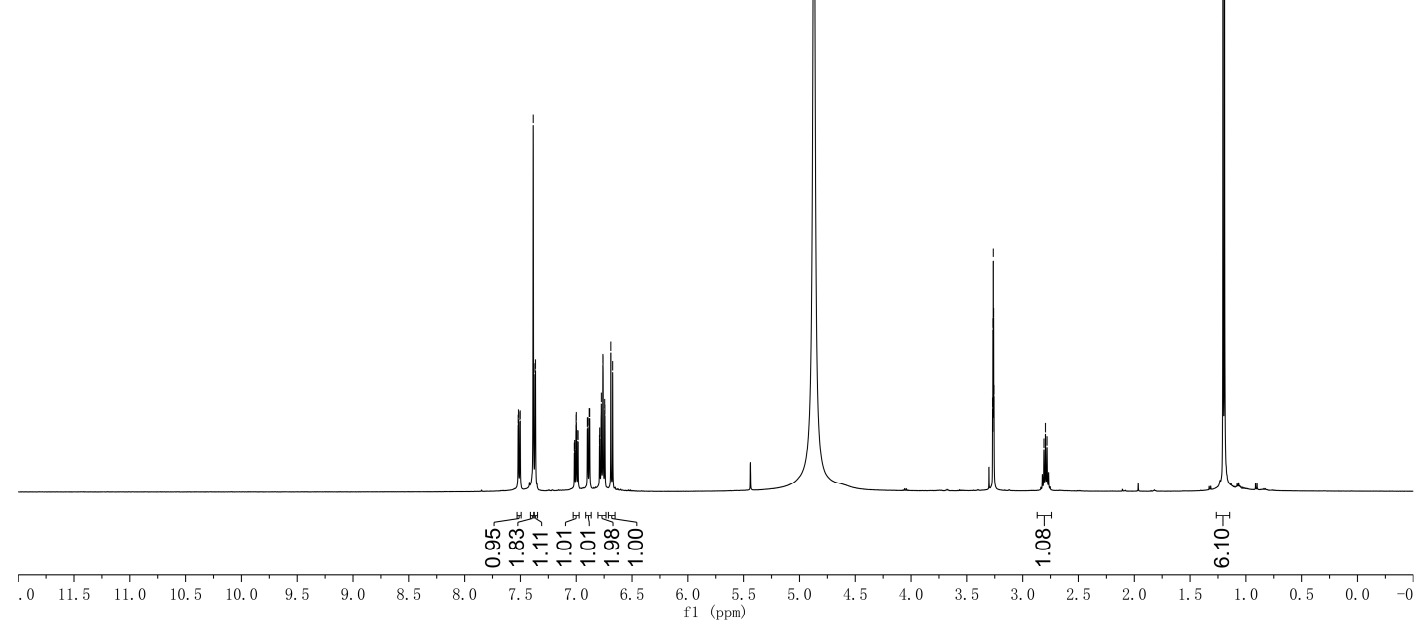

ZX200611A1
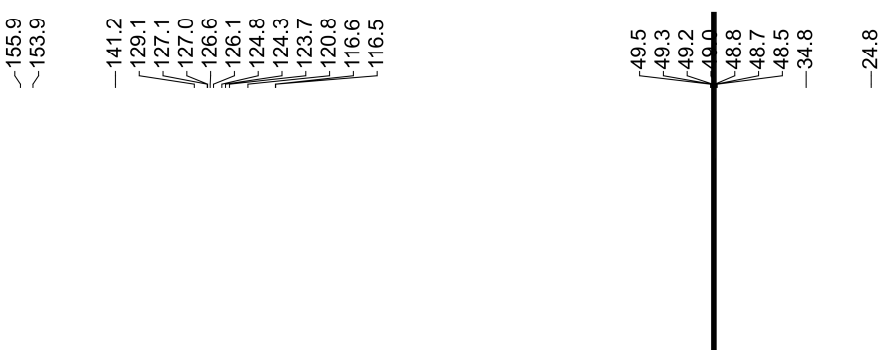

${ }^{13} \mathrm{C}$ NMR $\left(125 \mathrm{MHz}\right.$, Methanol- $\left.d_{4}\right)$
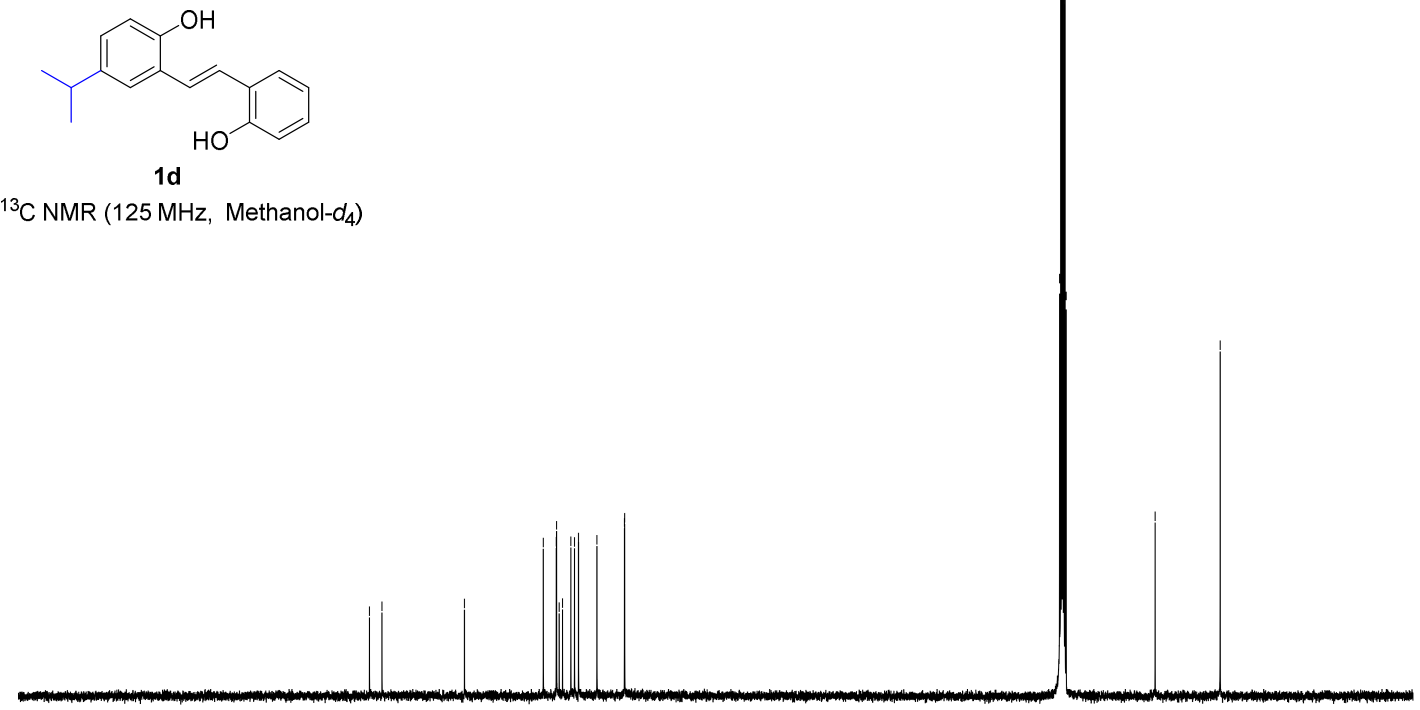

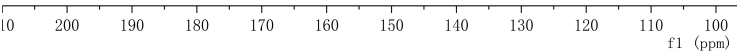




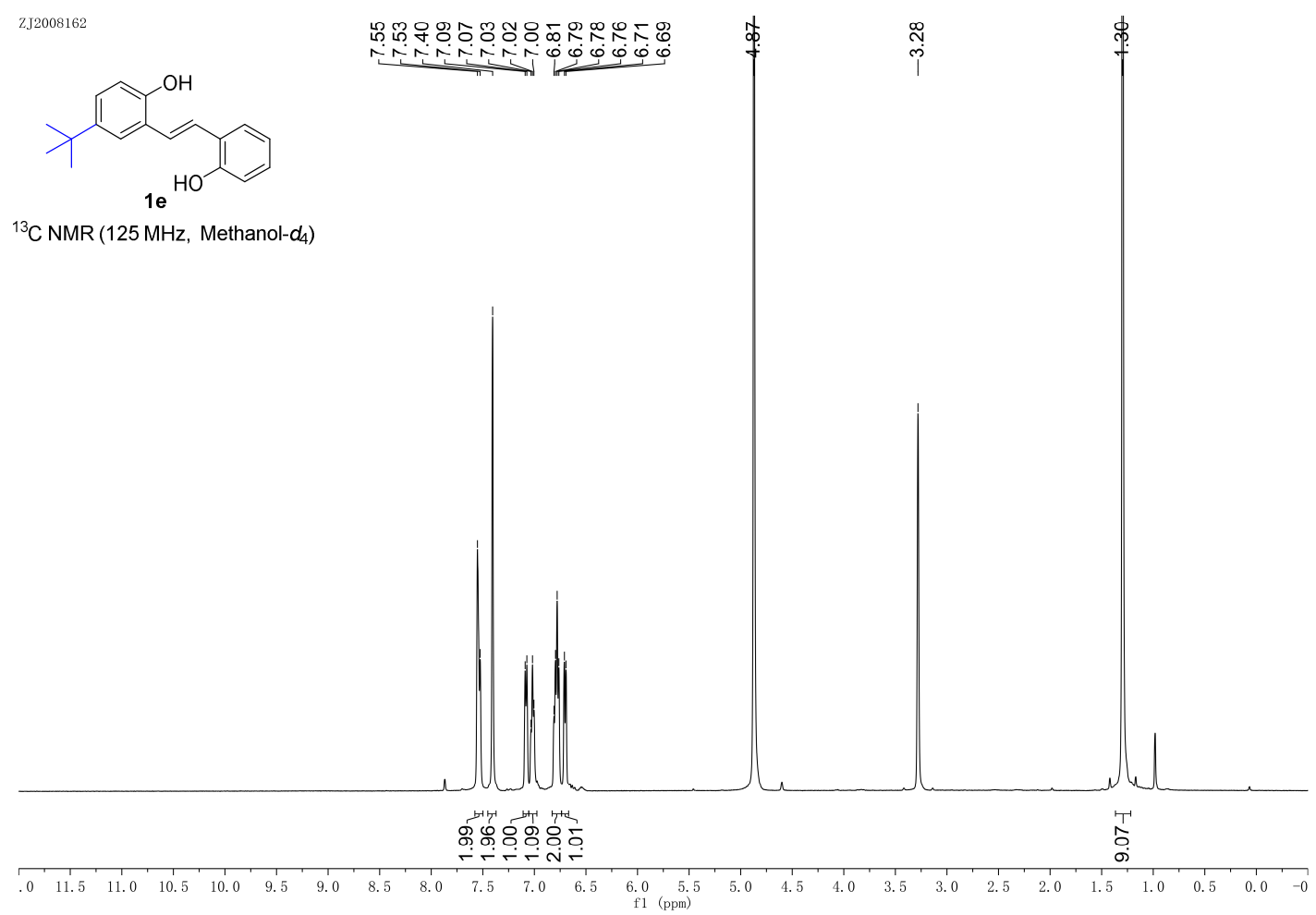

ZJ2008162
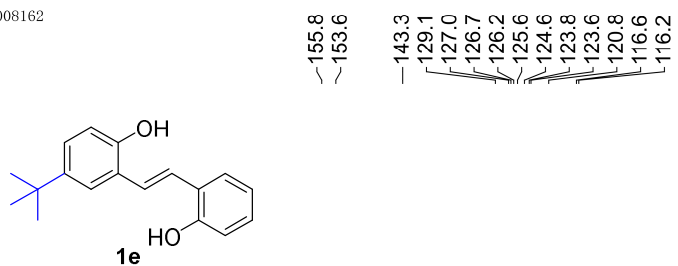

${ }^{13} \mathrm{C}$ NMR $\left(125 \mathrm{MHz}\right.$, Methanol- $\left.d_{4}\right)$

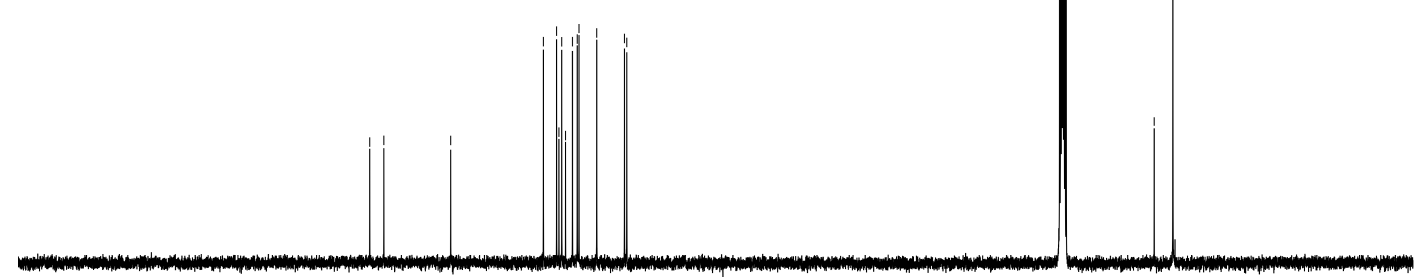

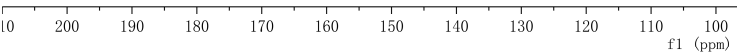



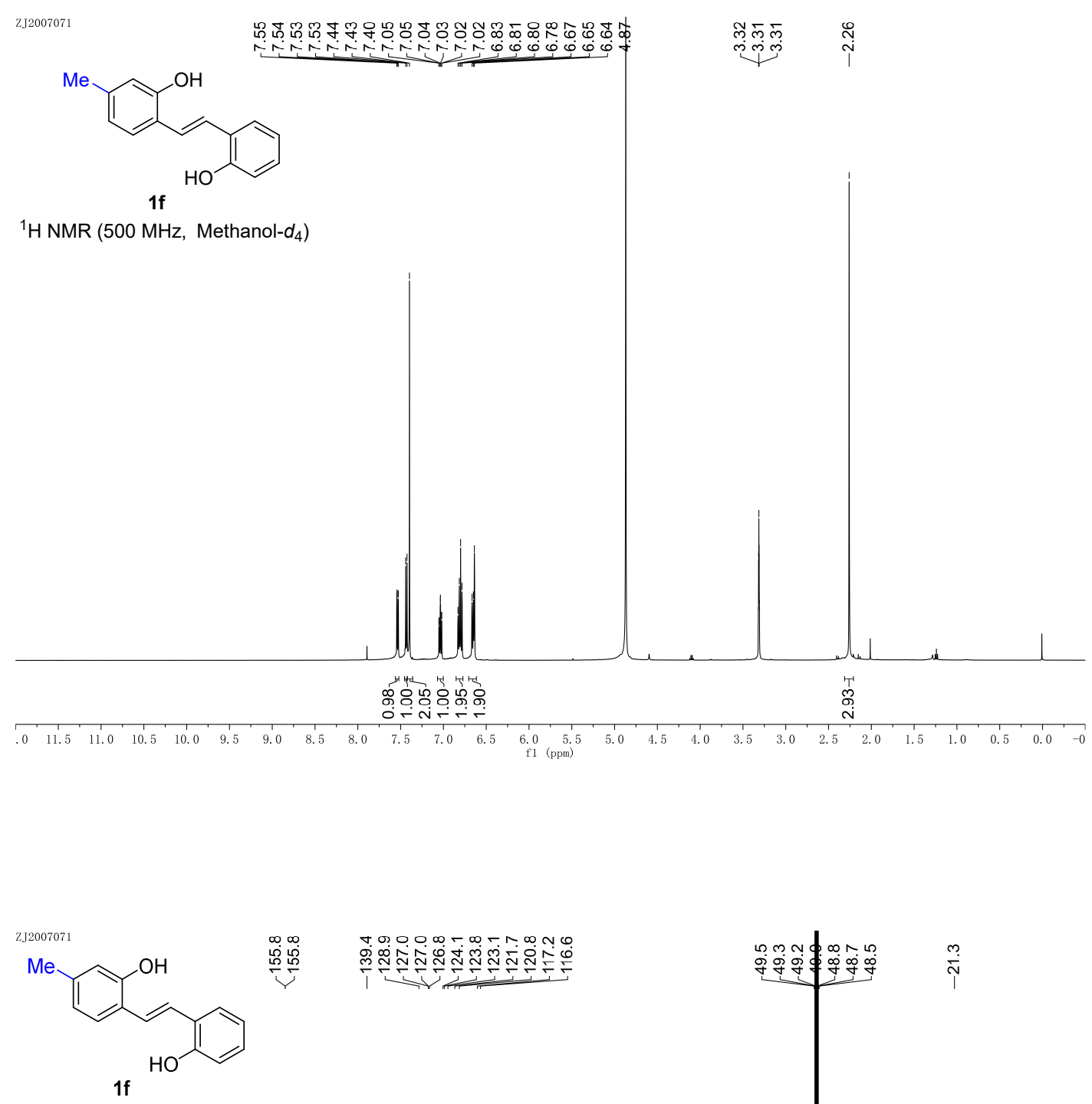

${ }^{13} \mathrm{C}$ NMR (125 MHz, Methanol- $\left.d_{4}\right)$
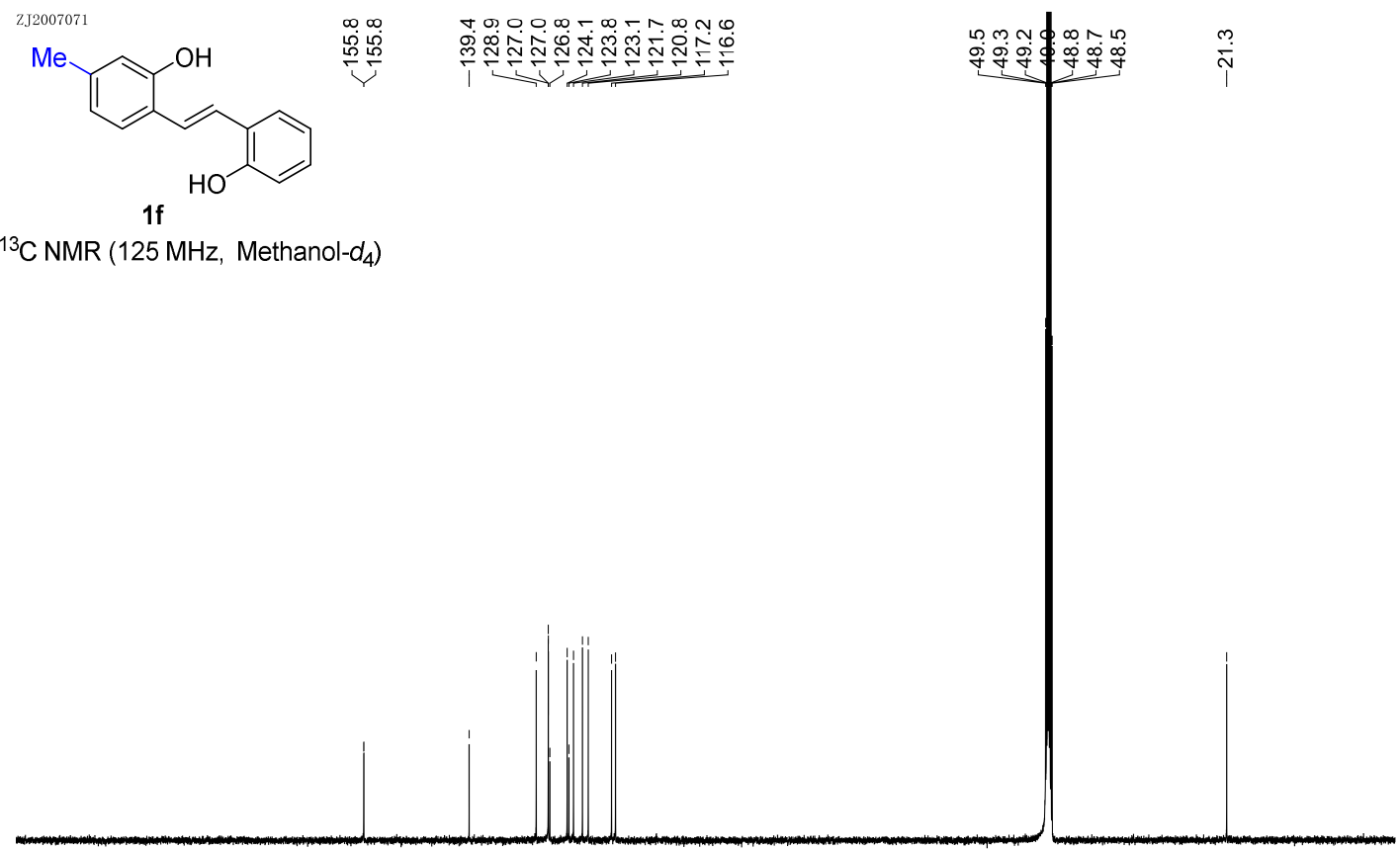

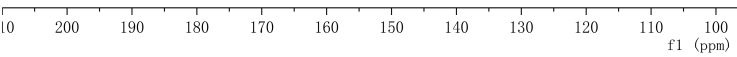




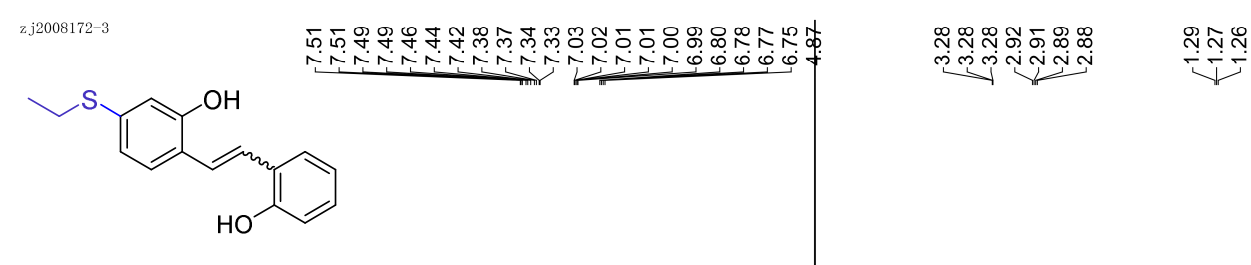

$1 \mathrm{~g}(Z / E, 1: 4)$

${ }^{1} \mathrm{H}$ NMR $\left(500 \mathrm{MHz}\right.$, Methanol- $\left.d_{4}\right)$
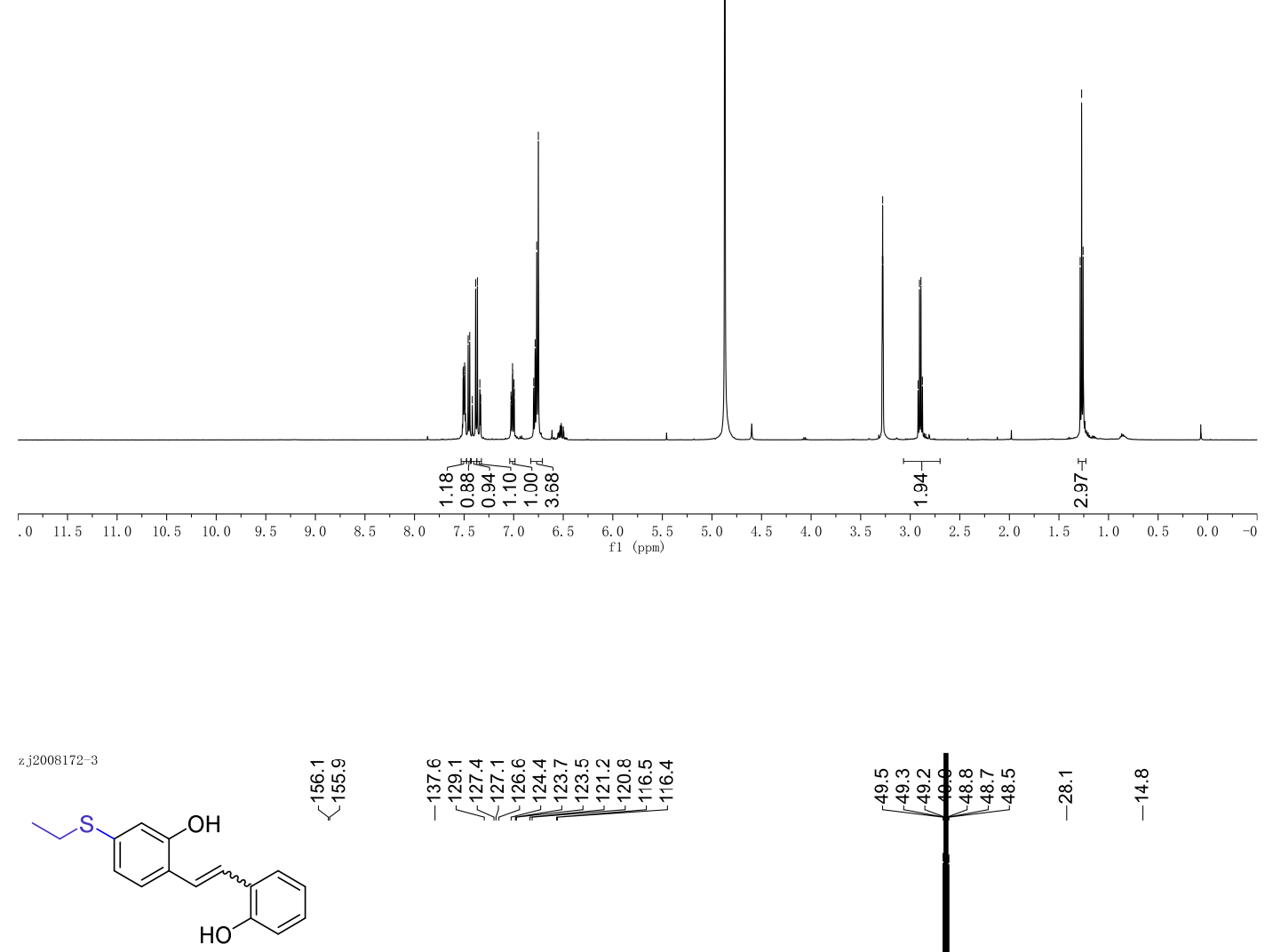

$1 \mathrm{~g}(Z / E, 1: 4)$

${ }^{13} \mathrm{C}$ NMR $\left(125 \mathrm{MHz}\right.$, Methanol- $\left.d_{4}\right)$

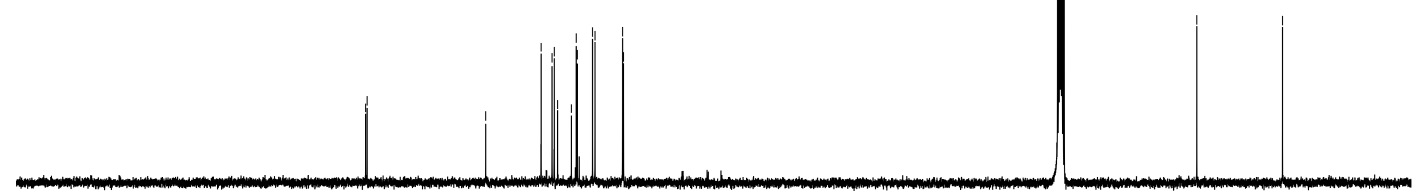

$\begin{array}{llllllllllll}10 & 200 & 190 & 180 & 170 & 160 & 150 & 140 & 130 & 120 & 110 & 1 \\ \mathrm{f} 1(\mathrm{pmm}) & 1\end{array}$ 


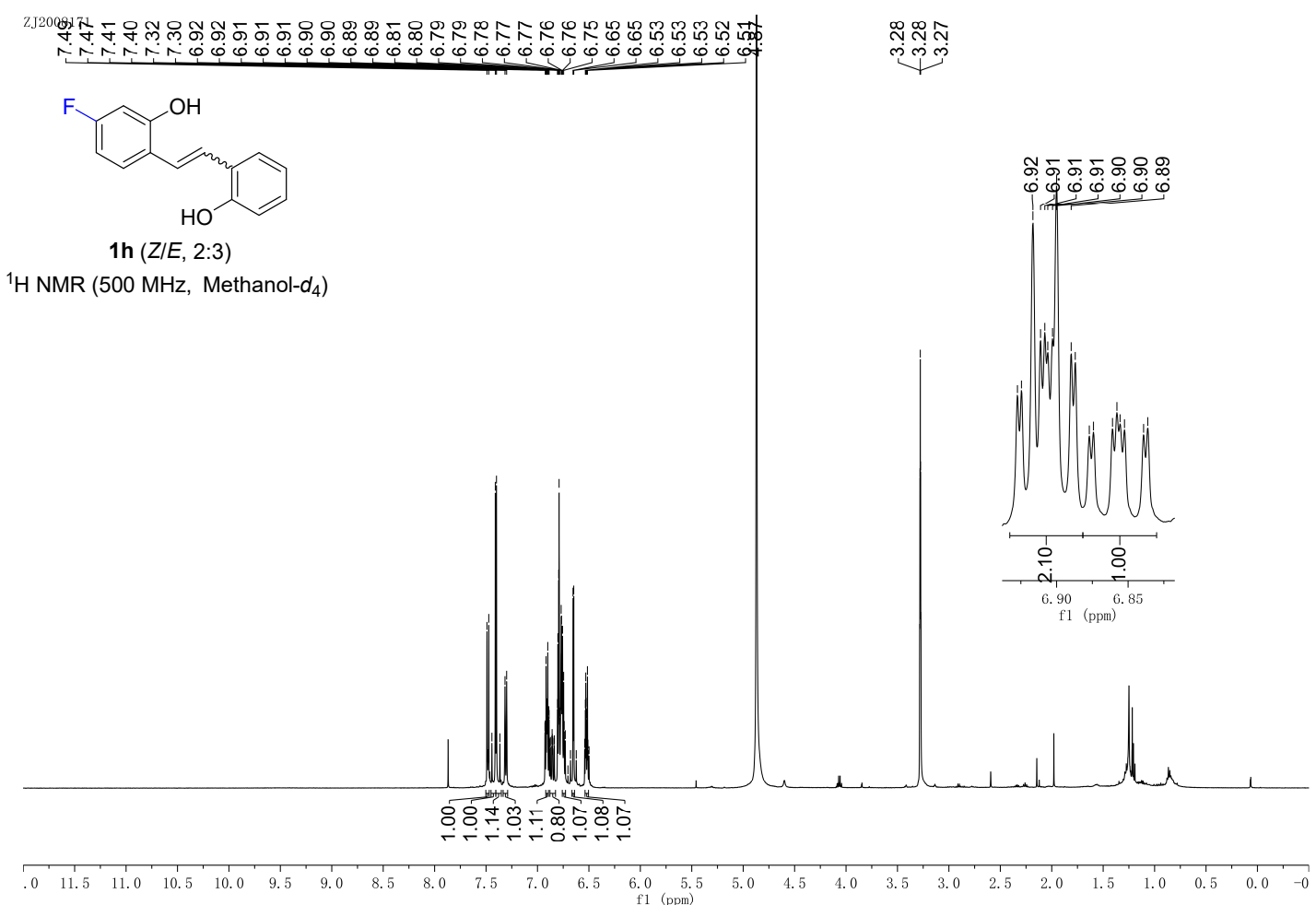

ZJ2008171

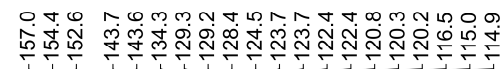

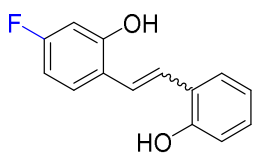

1h (ZIE, 2:3)

${ }^{13} \mathrm{C}$ NMR $\left(125 \mathrm{MHz}\right.$, Methanol- $\left.d_{4}\right)$

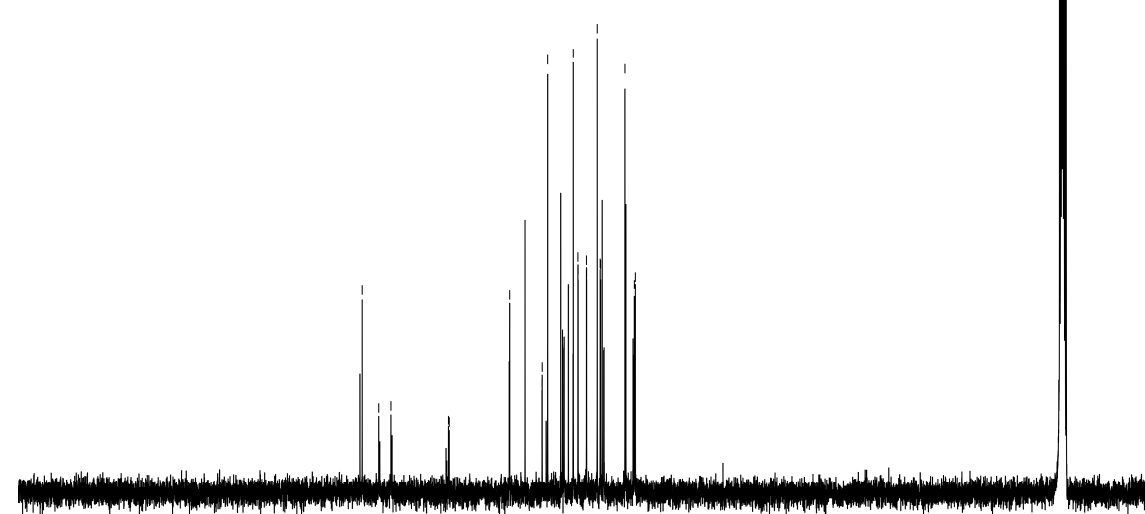

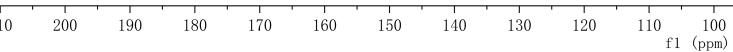


zj2008172F

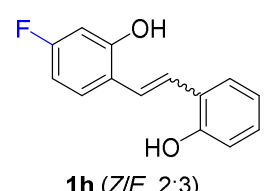

官官

1h (ZIE, 2:3)

${ }^{19} \mathrm{~F} \mathrm{NMR}\left(471 \mathrm{MHz}\right.$, Methanol- $\left.d_{4}\right)$

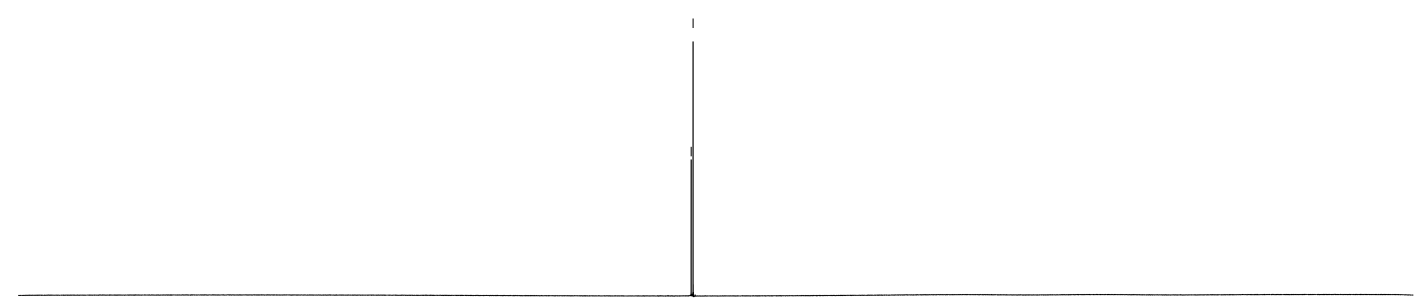

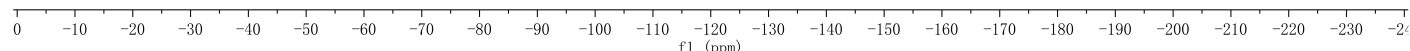



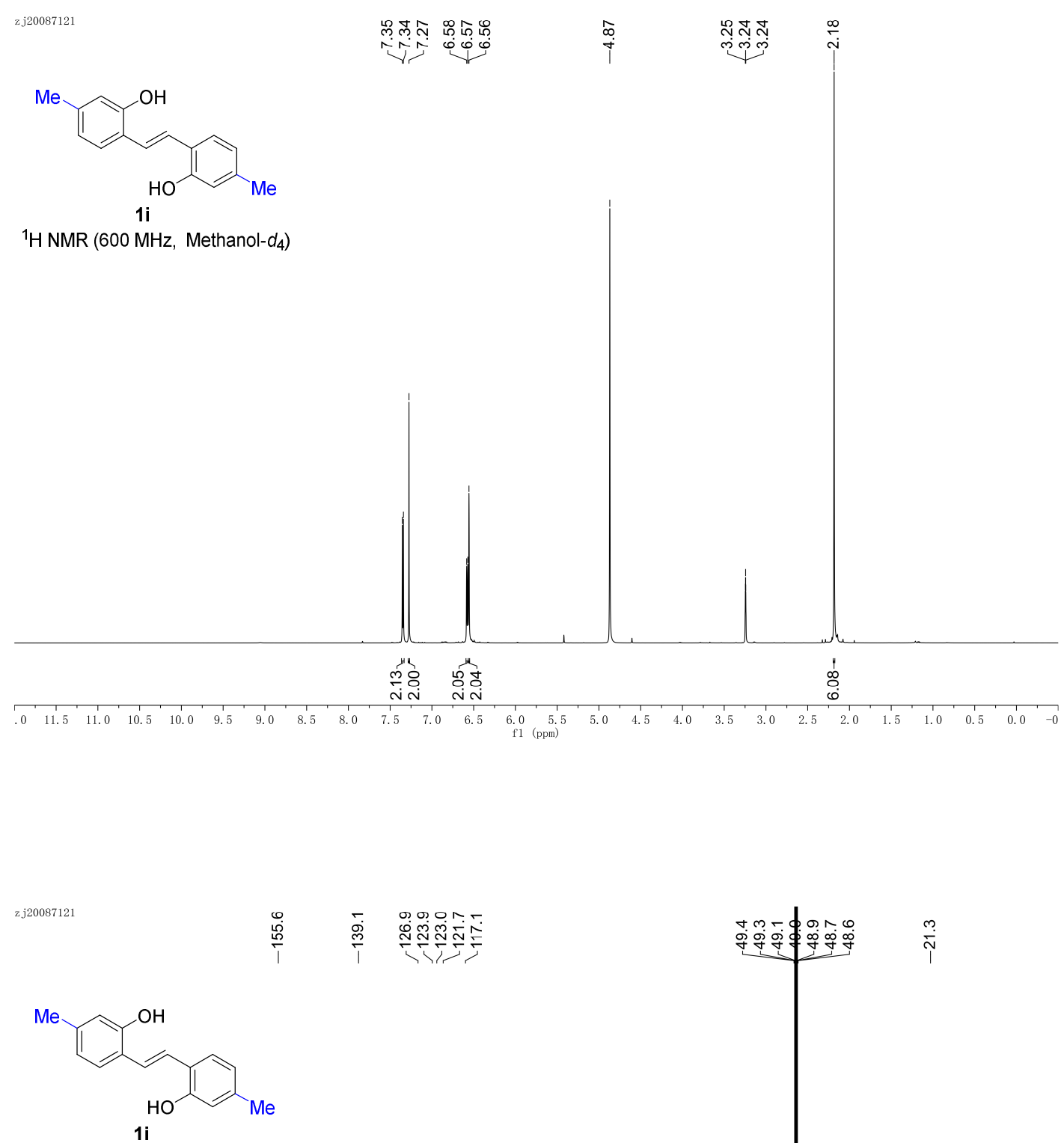

${ }^{13} \mathrm{C}$ NMR $\left(150 \mathrm{MHz}\right.$, Methanol- $\left.d_{4}\right)$
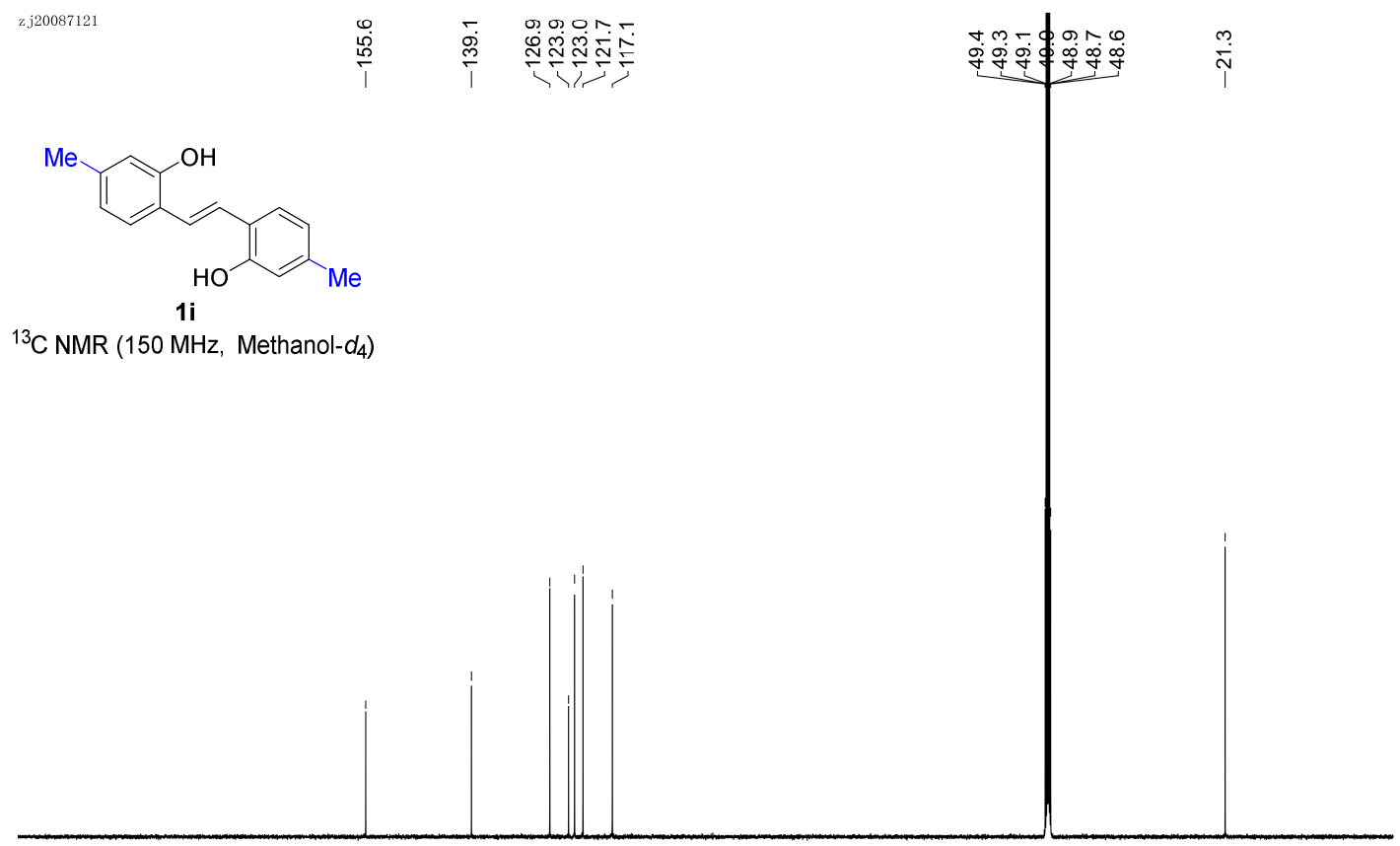

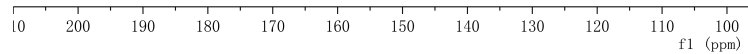



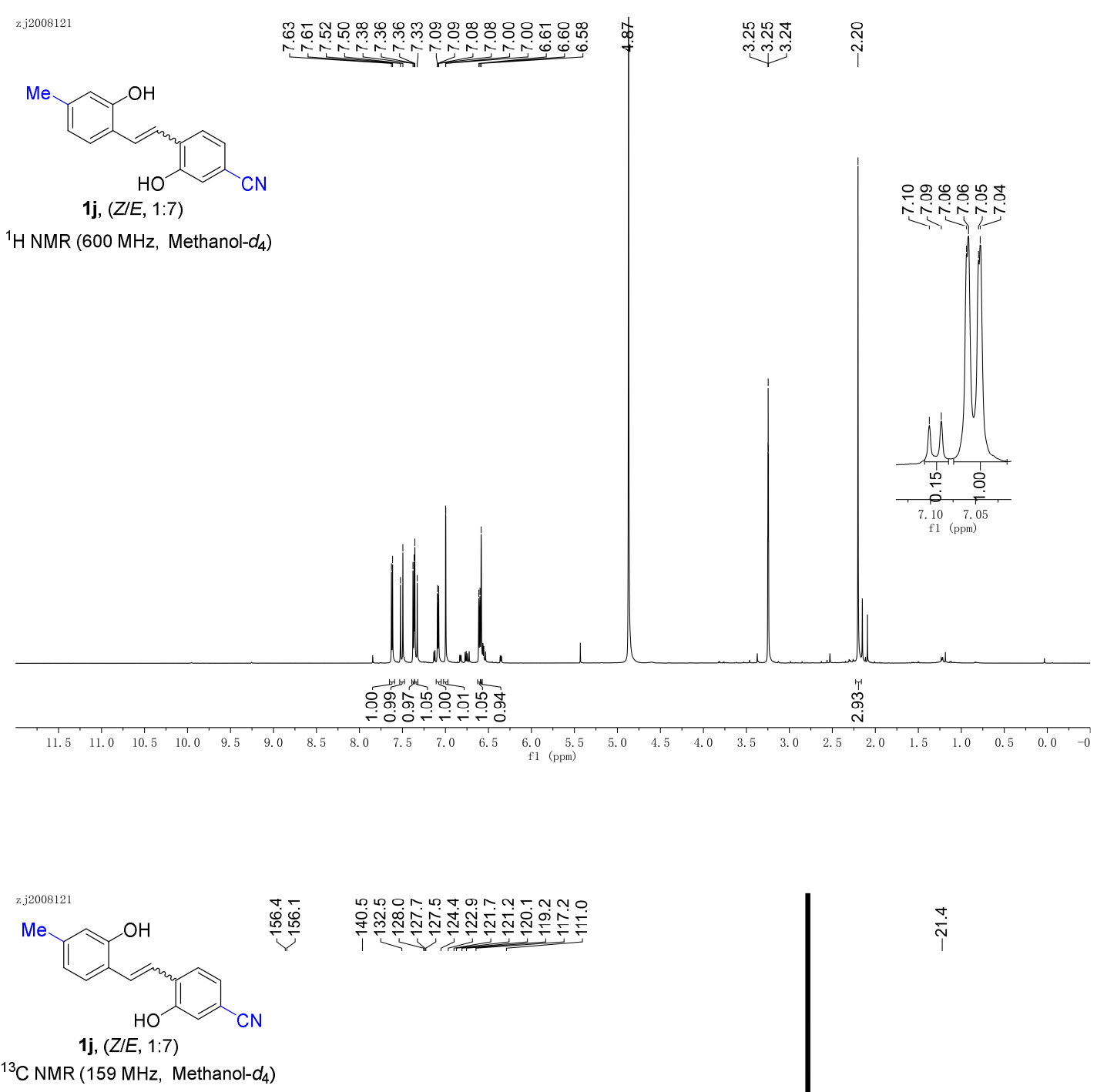

$\stackrel{\text { ì }}{\dot{\pi}}$

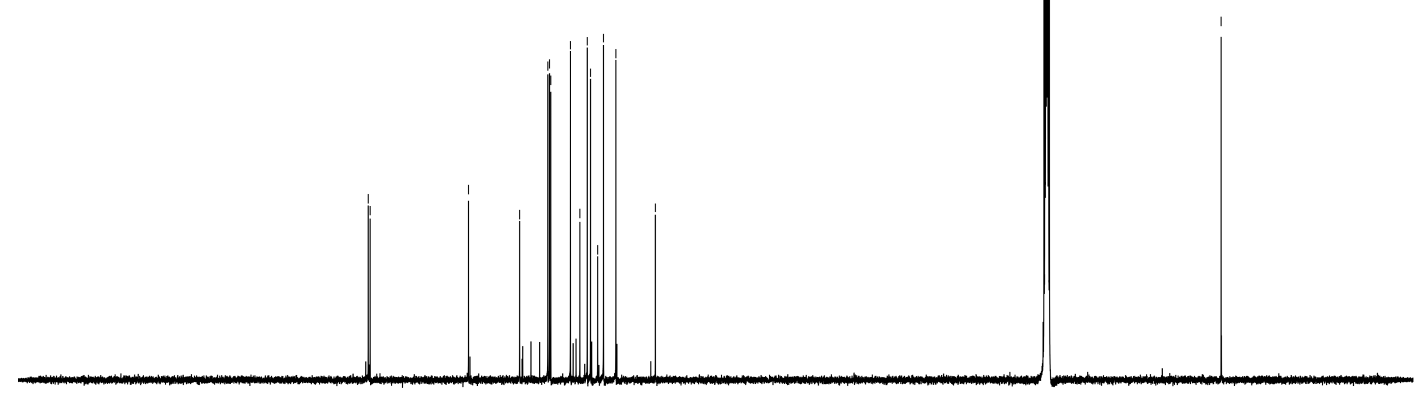

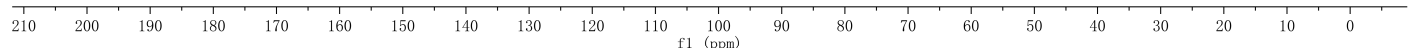




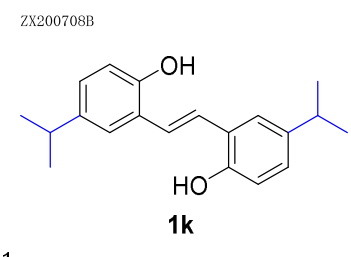

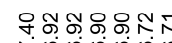

i

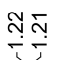

i 00000

${ }^{1} \mathrm{H}$ NMR $\left(500 \mathrm{MHz}\right.$, Methanol- $\left.d_{4}\right)$
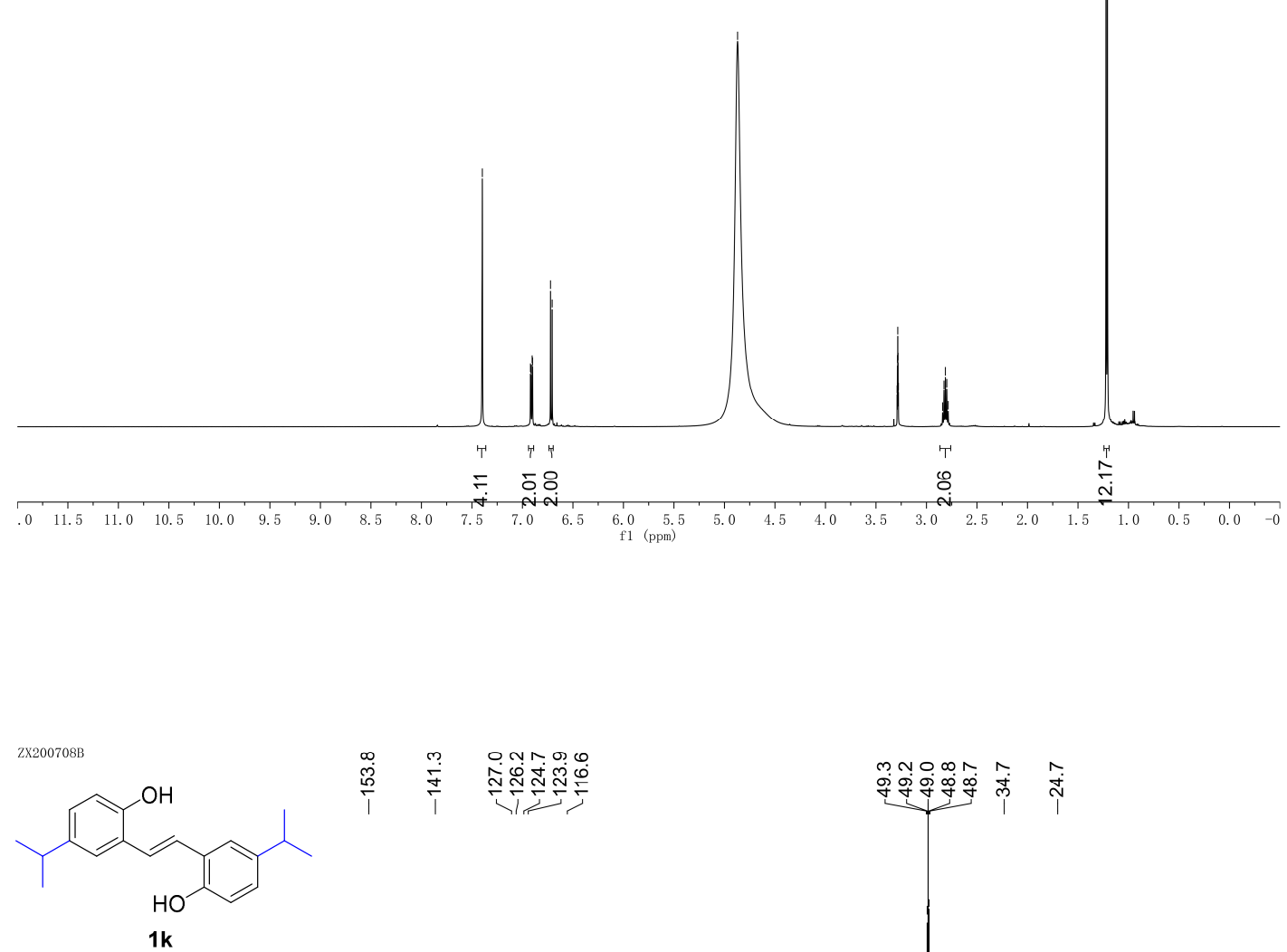

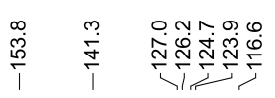

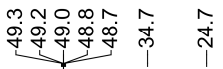

${ }^{13} \mathrm{C}$ NMR (125 MHz, Methanol-d 4 )

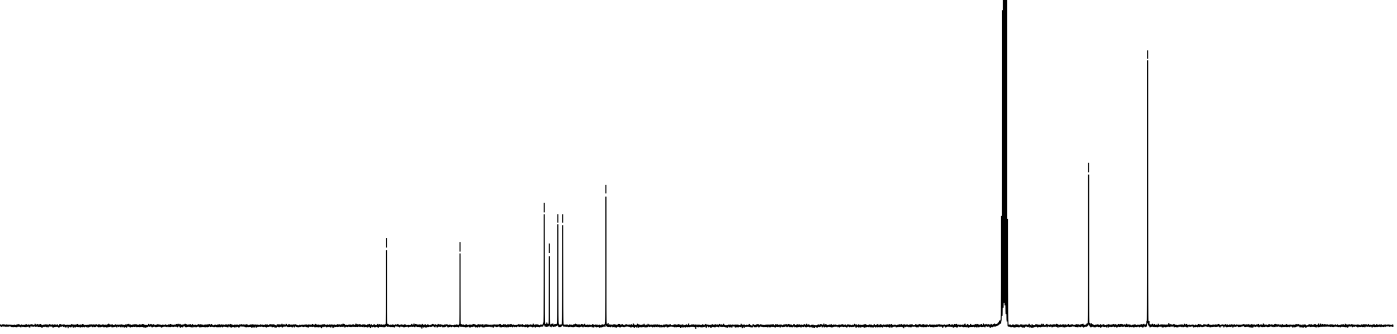

$\begin{array}{llllllllllllllllllllllllll}1 & 1 \\ 210 & 200 & 190 & 180 & 170 & 160 & 150 & 140 & 130 & 120 & 110 & 100 & 90 & 80 & 70 & 60 & 50 & 40 & 30 & 20 & 10 & 0 & -10\end{array}$ 

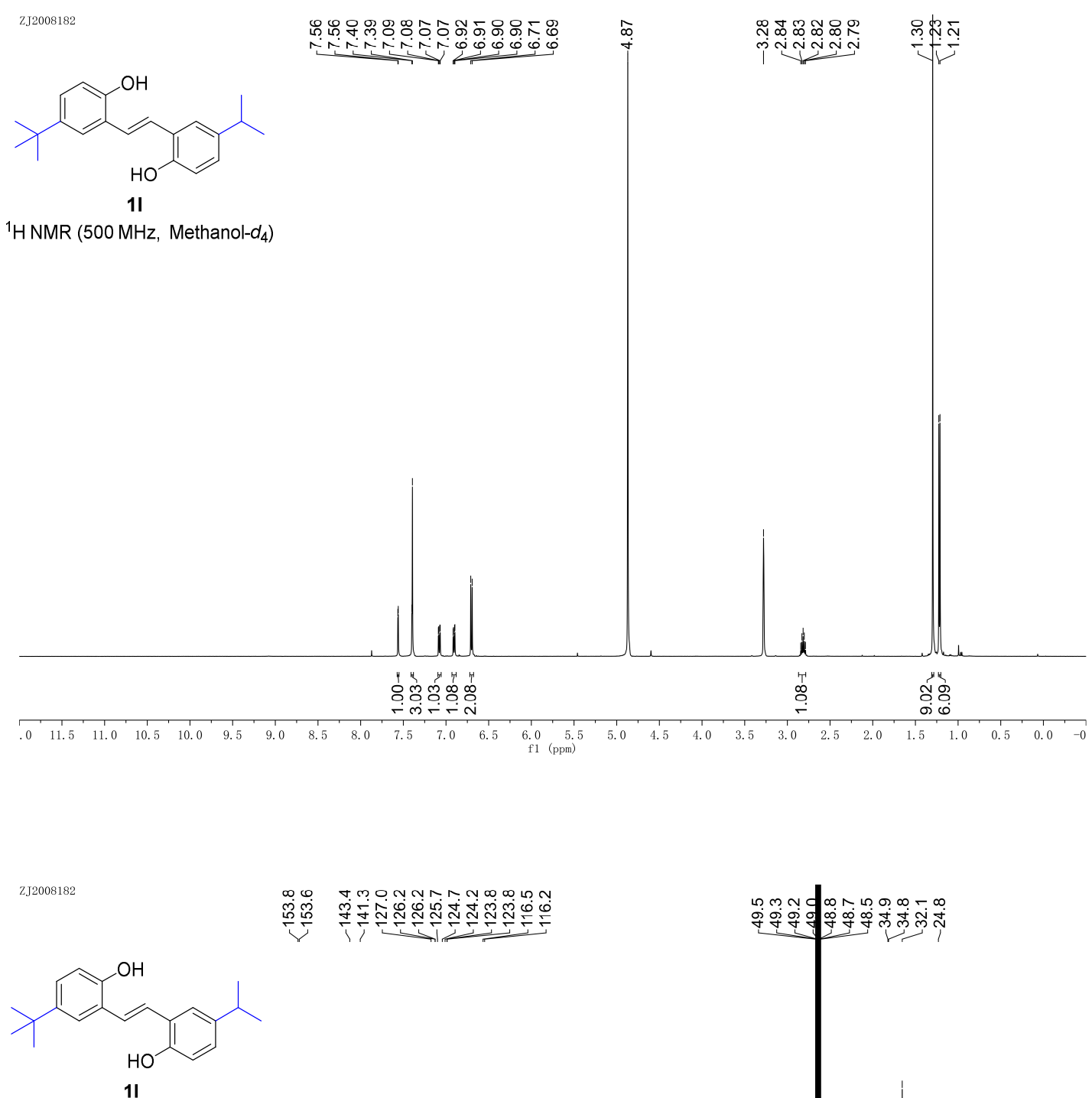

至

${ }^{13} \mathrm{C}$ NMR $\left(125 \mathrm{MHz}\right.$, Methanol- $\left.d_{4}\right)$

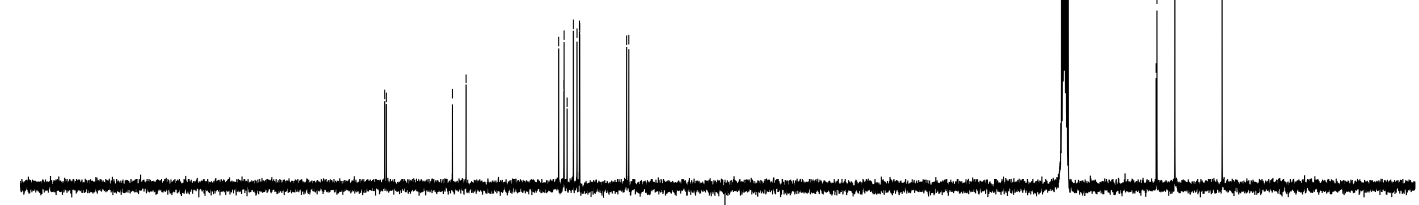

$\begin{array}{lllllllllllllllllllllllll}10 & 200 & 190 & 180 & 170 & 160 & 150 & 140 & 130 & 120 & 110 & 100 & 90 & 80 & 70 & 60 & 50 & 40 & 30 & 20 & 10 & 0\end{array}$ 

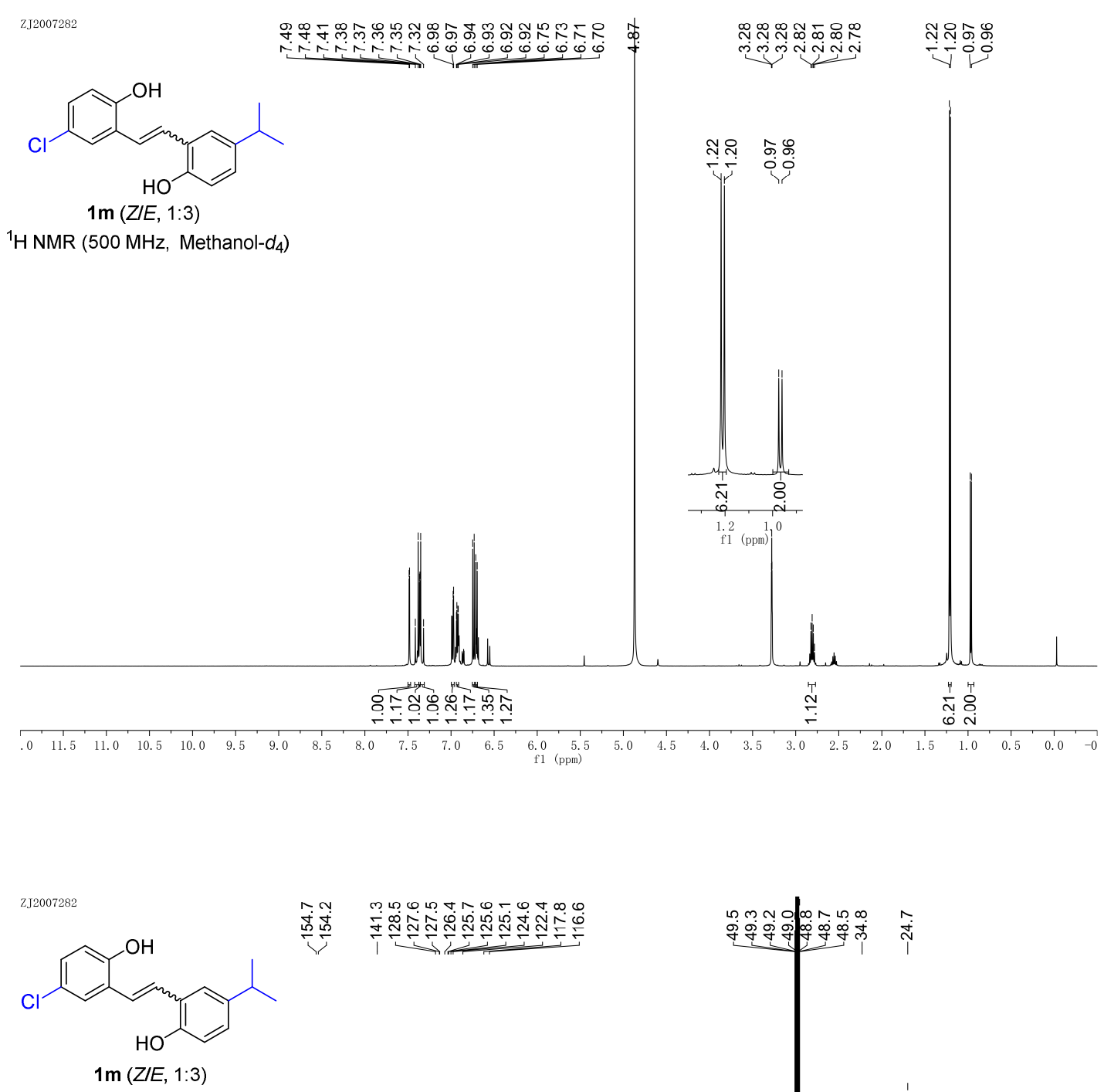

${ }^{13} \mathrm{C}$ NMR (125 MHz, Methanol-d $\left.d_{4}\right)$

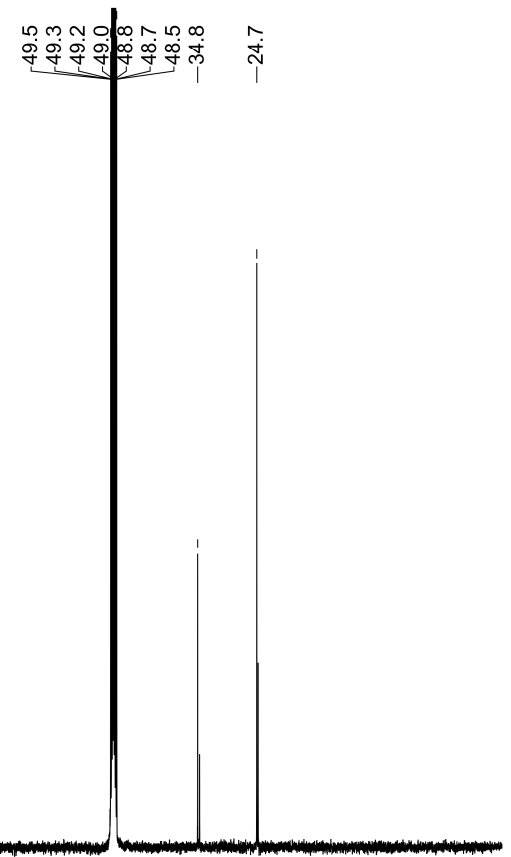



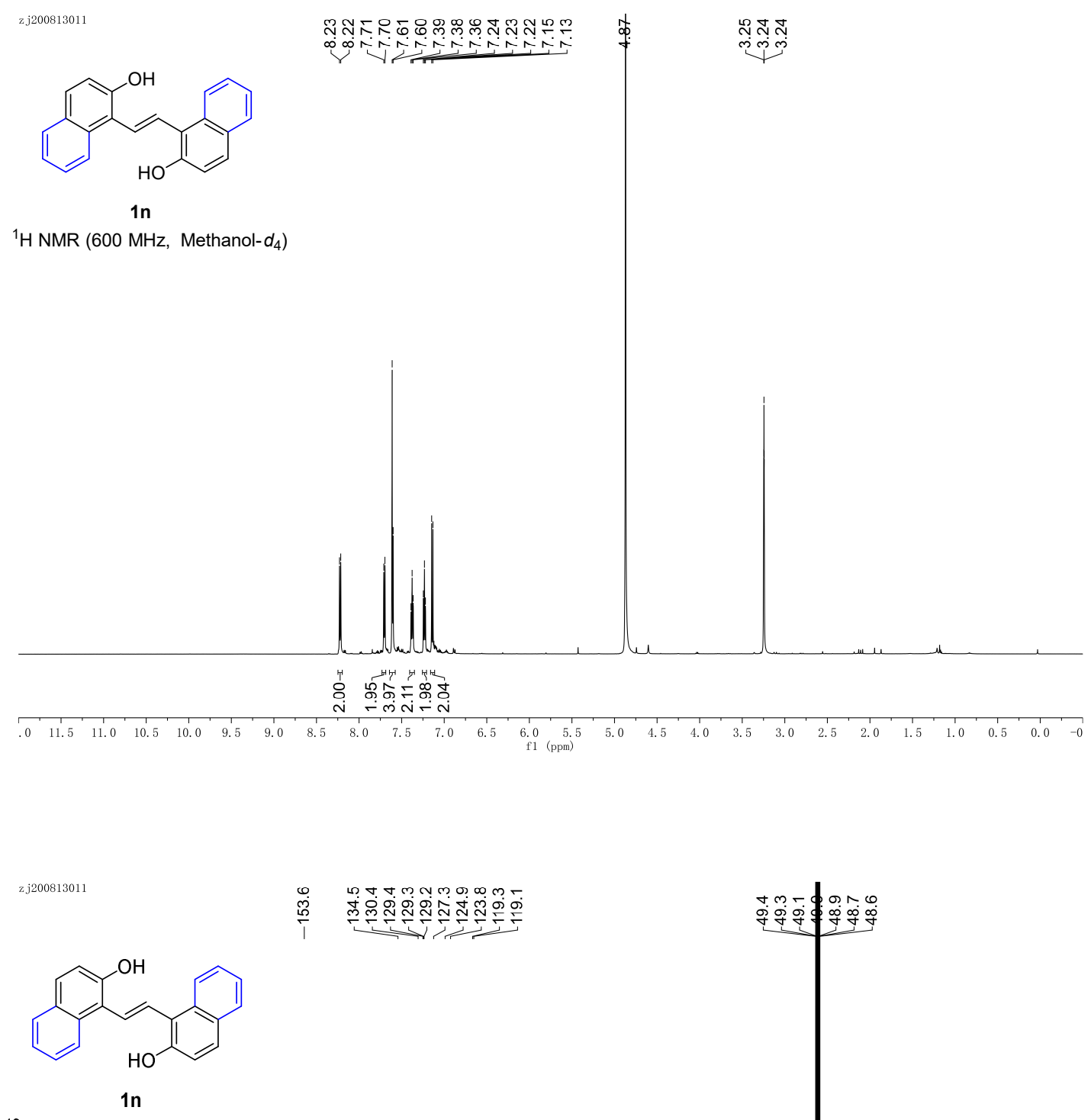

${ }^{13} \mathrm{C}$ NMR $\left(150 \mathrm{MHz}\right.$, Methanol- $\left.d_{4}\right)$

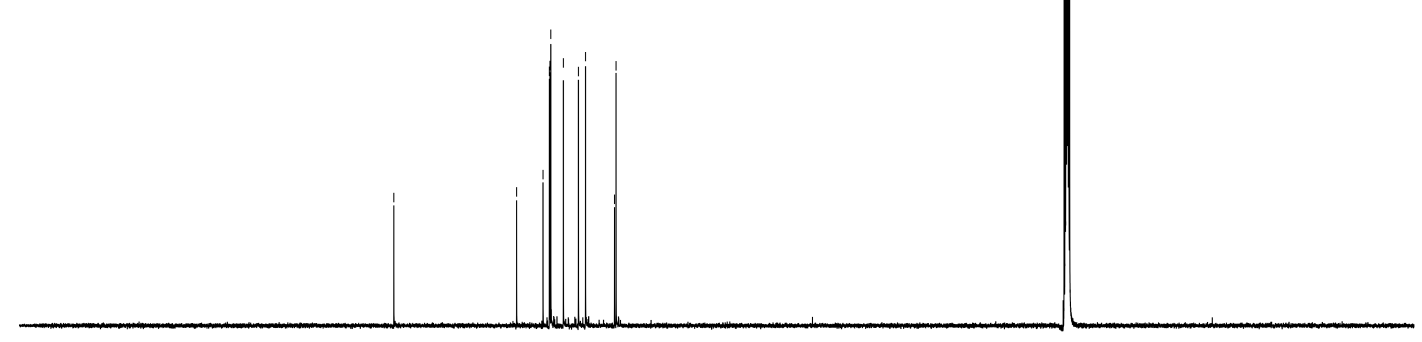

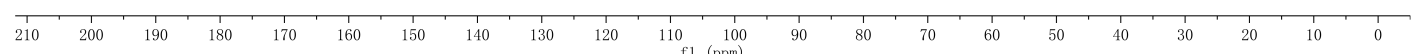



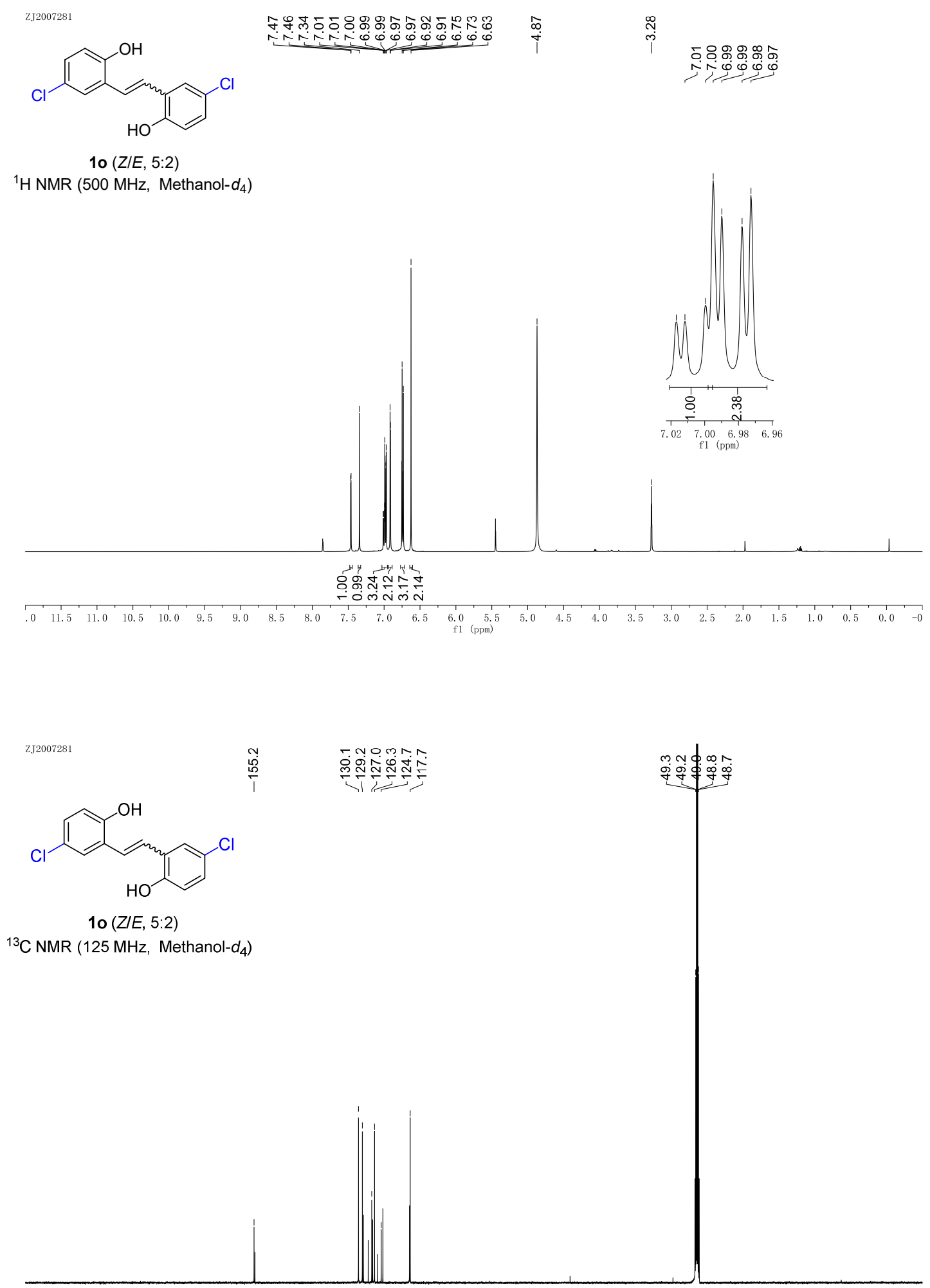

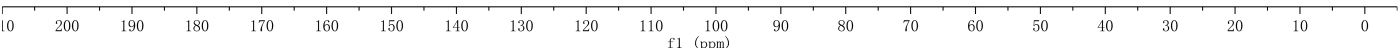



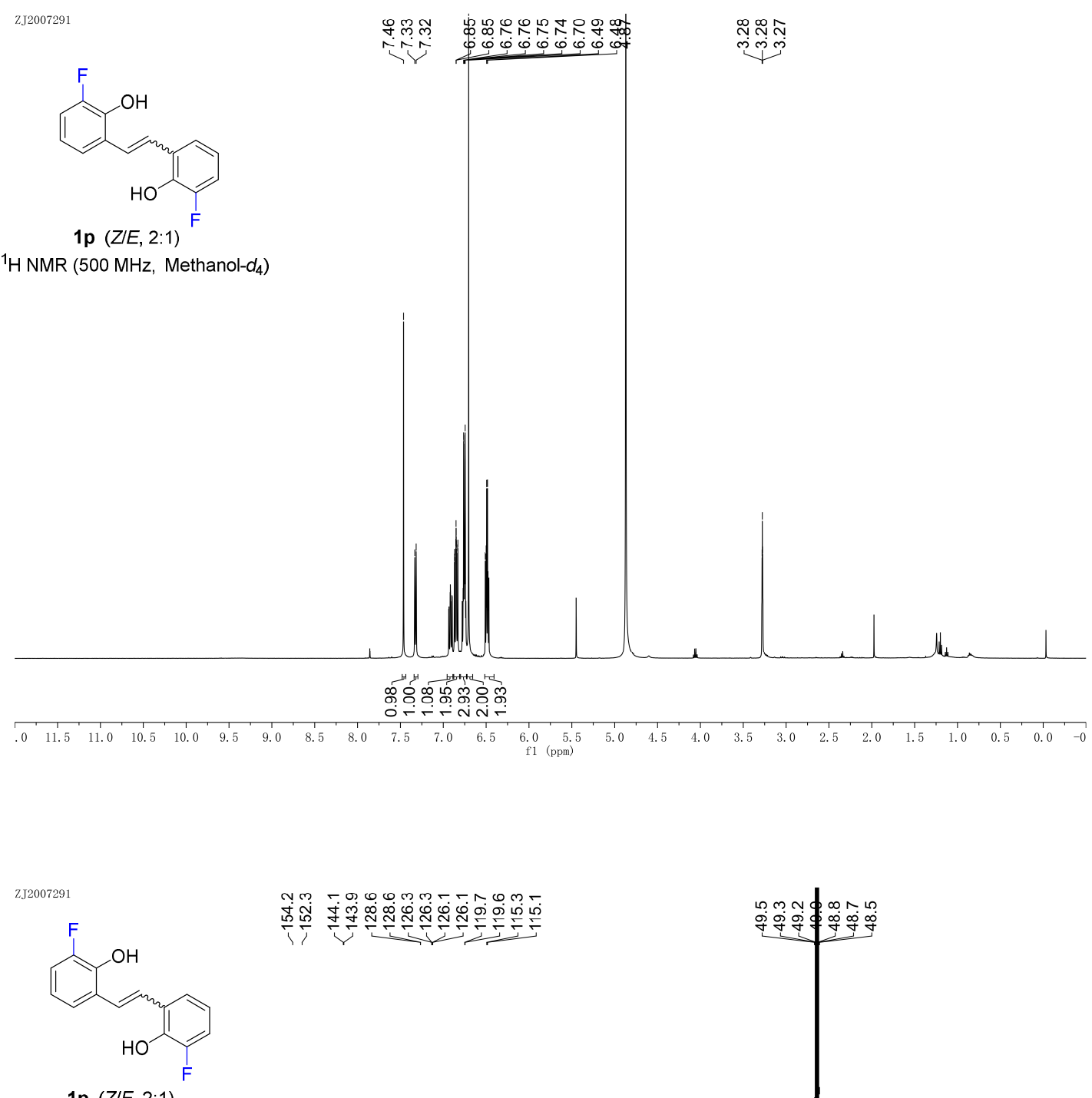

$1 p(Z / E, 2: 1)$

${ }^{13} \mathrm{C}$ NMR $\left(125 \mathrm{MHz}\right.$, Methanol- $\left.d_{4}\right)$

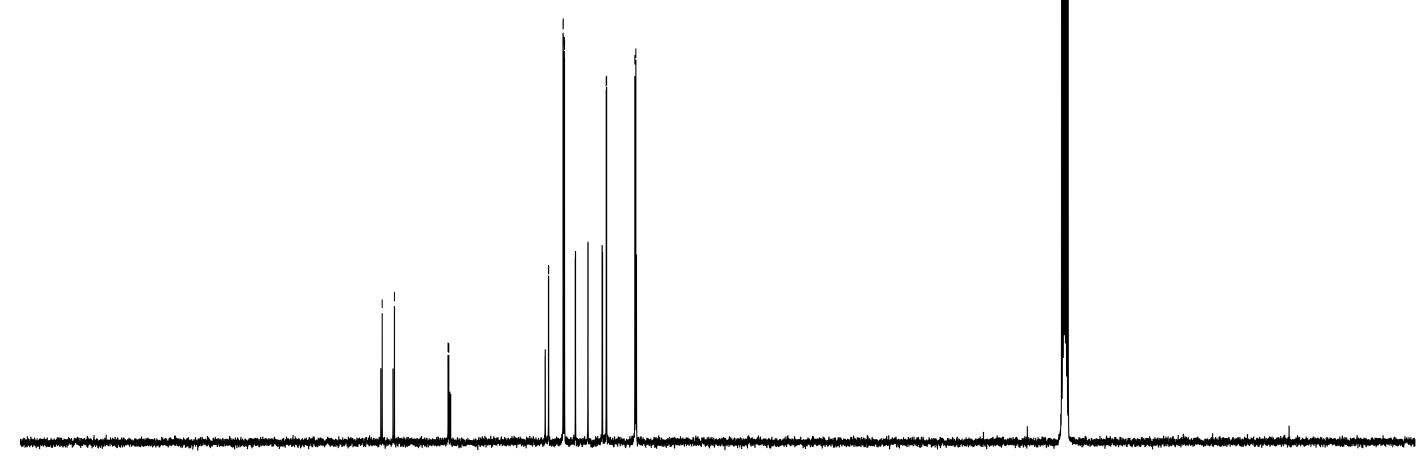

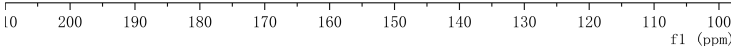




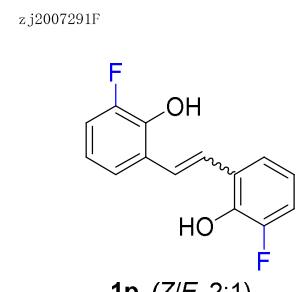

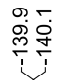

${ }^{19} \mathrm{~F}$ NMR (471 MHz, Methanol-d 4 )

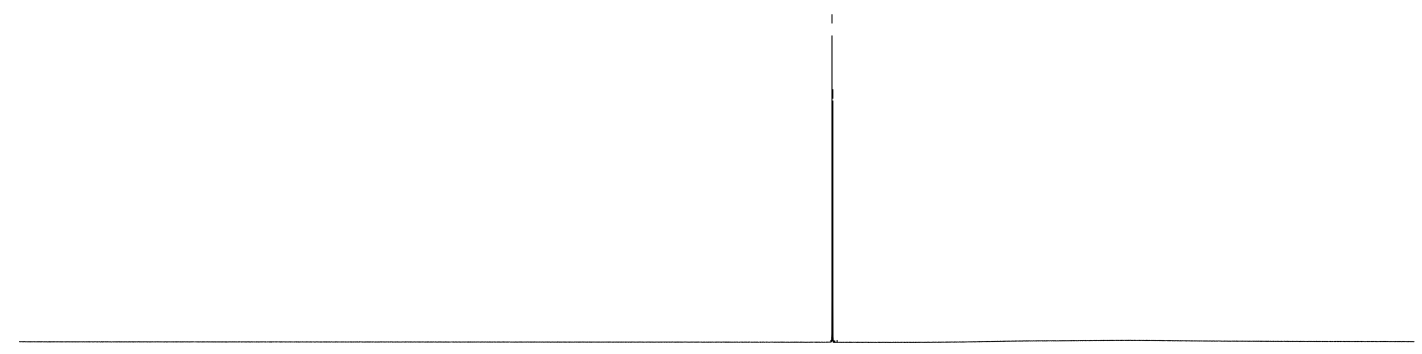

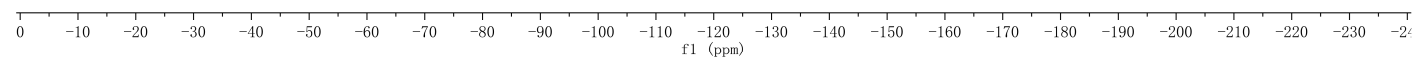


2J2007292

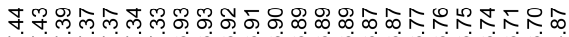

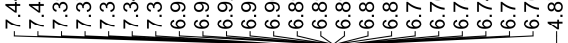

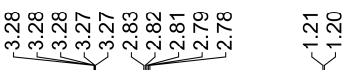<smiles>CC(C)c1ccc(O)c(/C=C/c2cccc(F)c2O)c1</smiles>

${ }^{1} \mathrm{H}$ NMR $\left(500 \mathrm{MHz}\right.$, Methanol- $\left.d_{4}\right)$

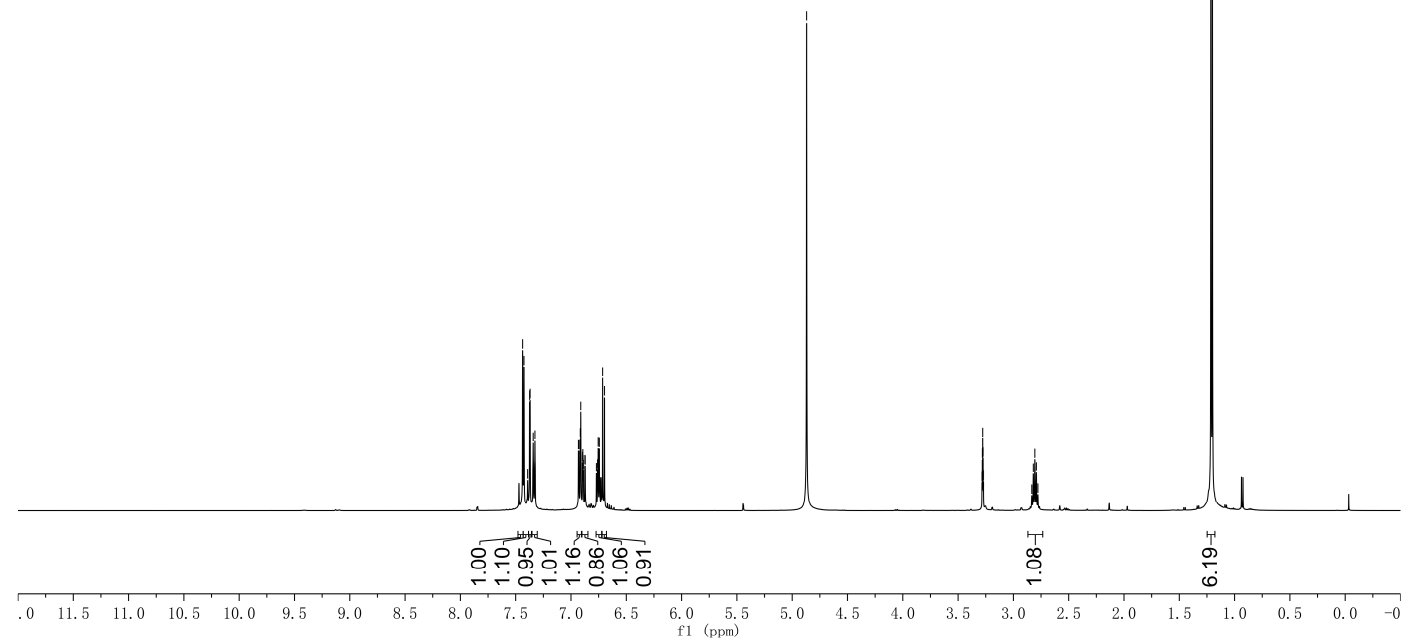

2J2007292

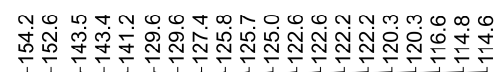

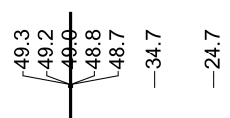<smiles>CC(C)c1ccc(O)c(C=Cc2cccc(O)c2O)c1</smiles>

${ }^{13} \mathrm{C}$ NMR $\left(125 \mathrm{MHz}\right.$, Methanol- $\left.d_{4}\right)$
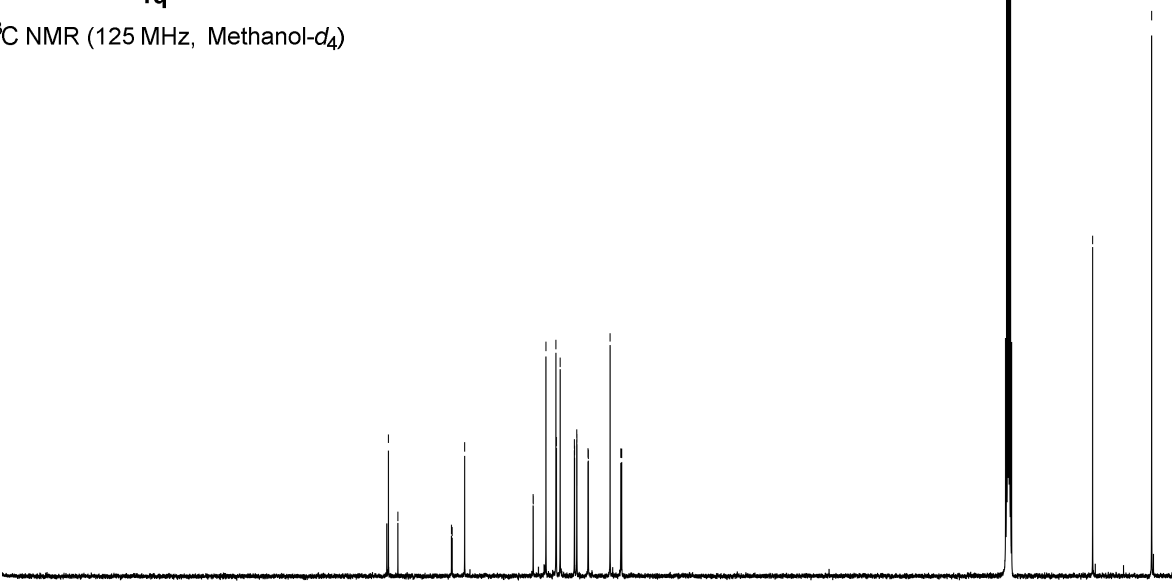
zj2007292F<smiles>CC(C)c1ccc(O)c(/C=C/c2cccc(O)c2O)c1</smiles>

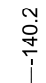

${ }^{19}$ F NMR (471 MHz, Methanol- $\left.d_{4}\right)$

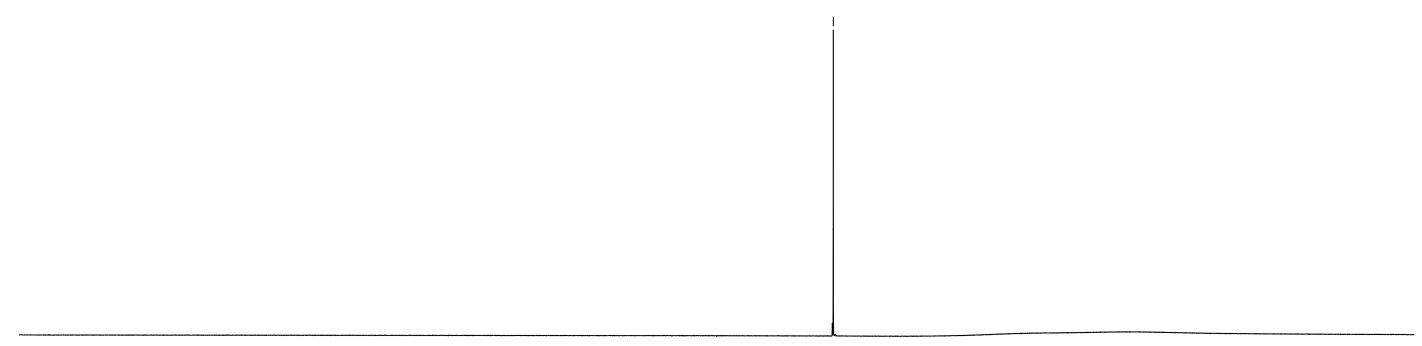

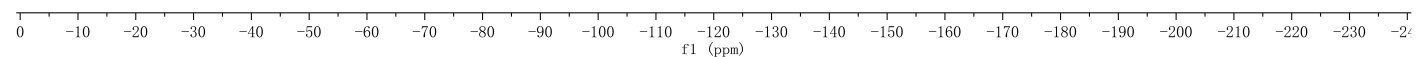




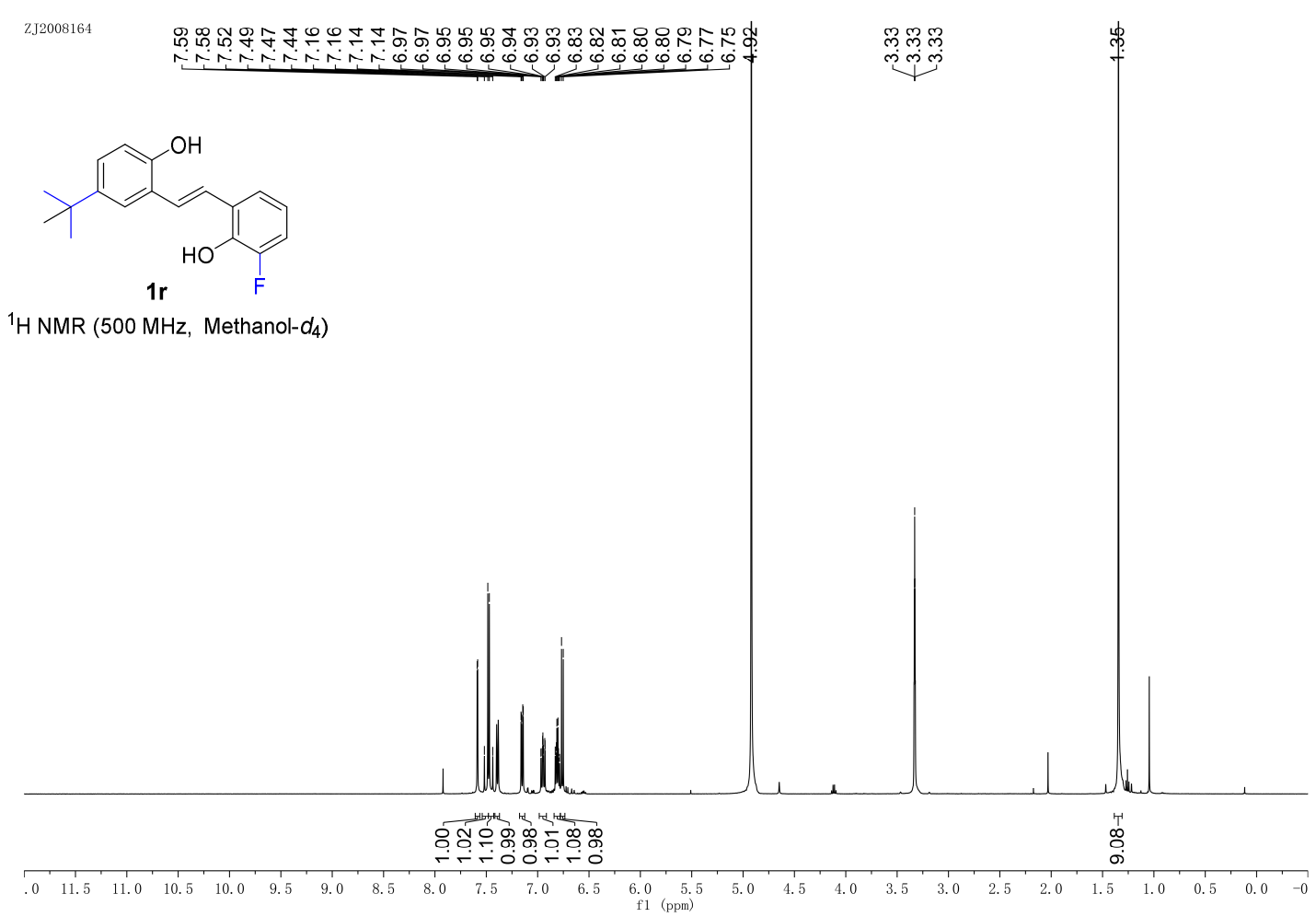

ZJ2008164

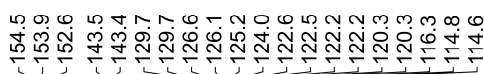

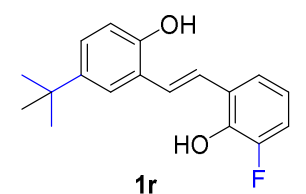

${ }^{1} \mathrm{C}$ NMR $(125 \mathrm{MHz}$, Methanol-d $)$
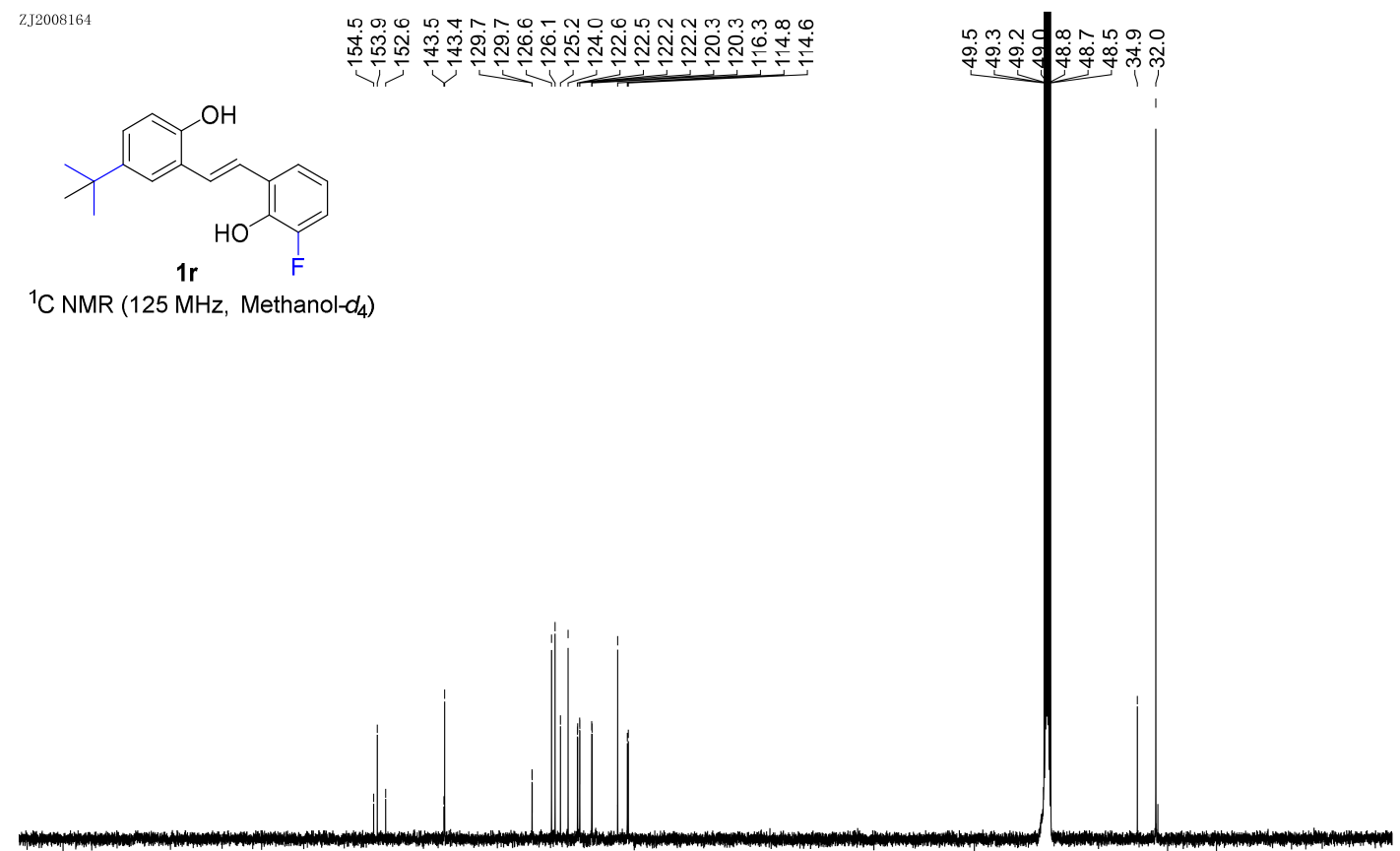

$\begin{array}{llllllllllll}10 & 200 & 190 & 180 & 170 & 160 & 150 & 140 & 130 & 120 & 110 & 1 \\ \mathrm{f} 1(\mathrm{pmm}) & 1\end{array}$ 
zj2008164F<smiles>CC(C)(C)c1ccc(O)c(/C=C/c2cccc(F)c2O)c1</smiles>

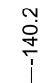

${ }^{19} \mathrm{~F}$ NMR (471 MHz, Methanol-d $\left.d_{4}\right)$

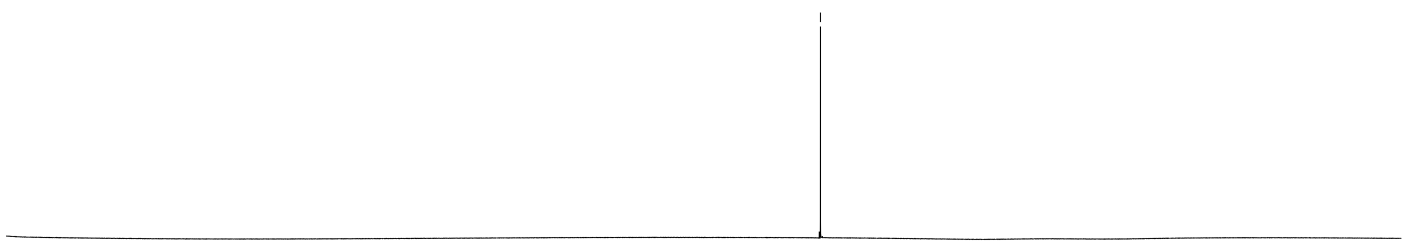

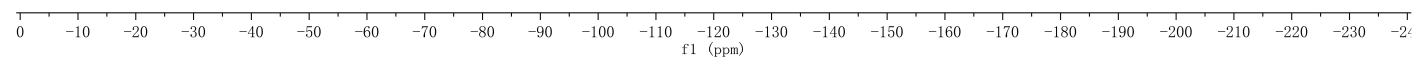




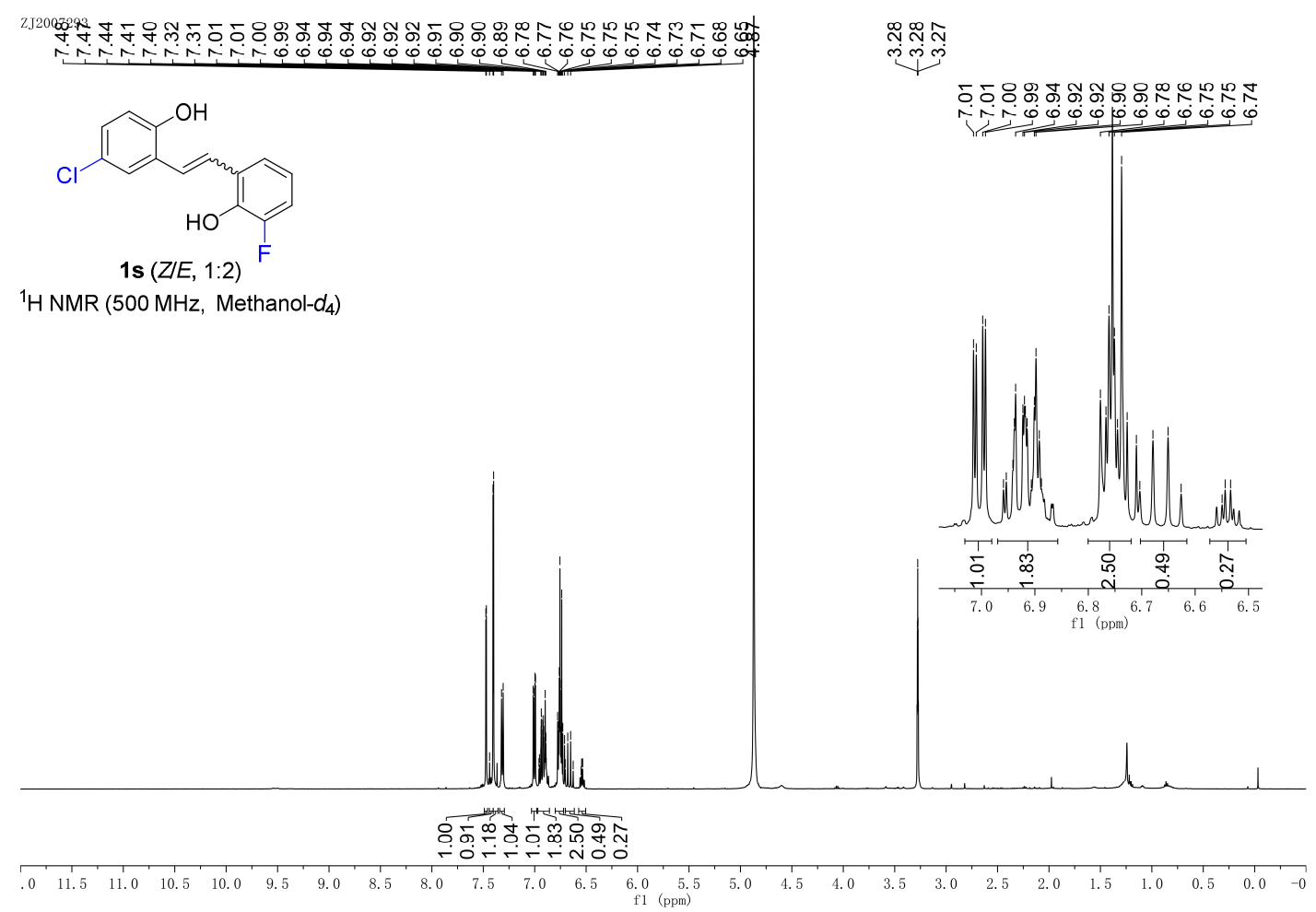

ZJ2007293
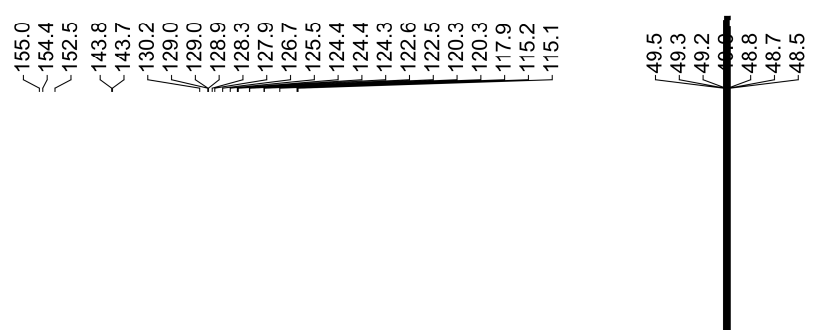

${ }^{13} \mathrm{C}$ NMR $\left(125 \mathrm{MHz}\right.$, Methanol-d $\left.d_{4}\right)$

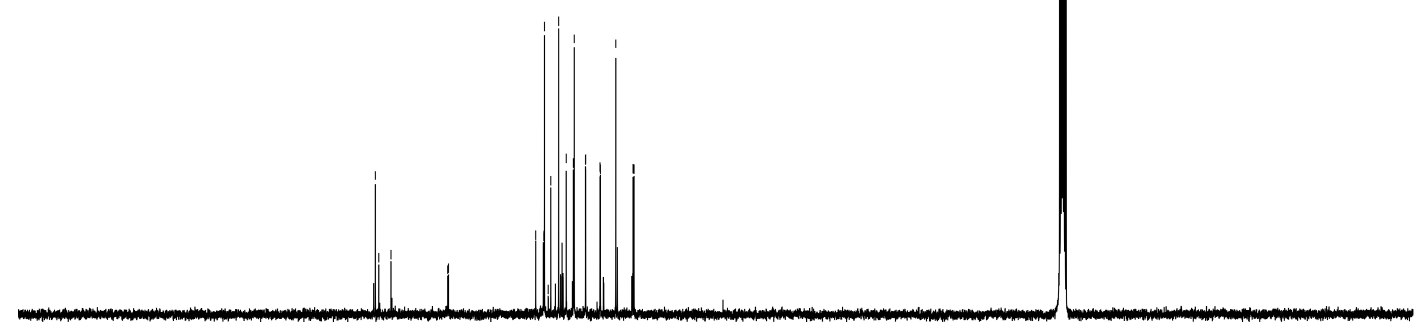

$\begin{array}{llllllllllll}10 & 200 & 190 & 180 & 170 & 160 & 150 & 140 & 130 & 120 & 110 & 1 \\ \mathrm{f} 1(\mathrm{pmm})\end{array}$ 
zj2007293F

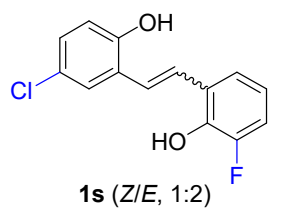

䑻字

${ }^{19} \mathrm{~F}$ NMR $\left(471 \mathrm{MHz}\right.$, Methanol- $\left.d_{4}\right)$

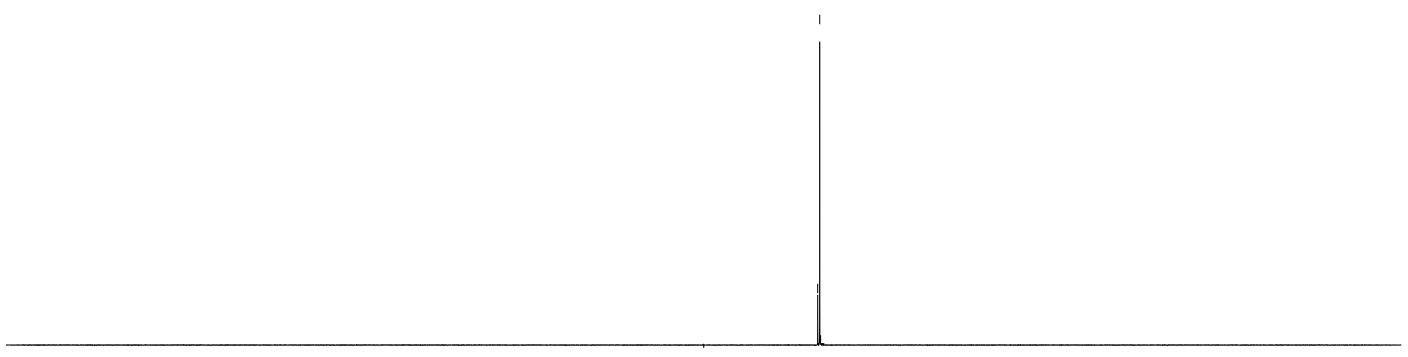

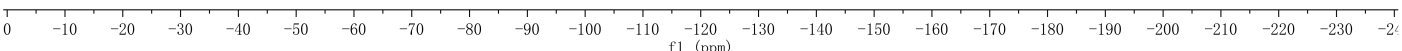



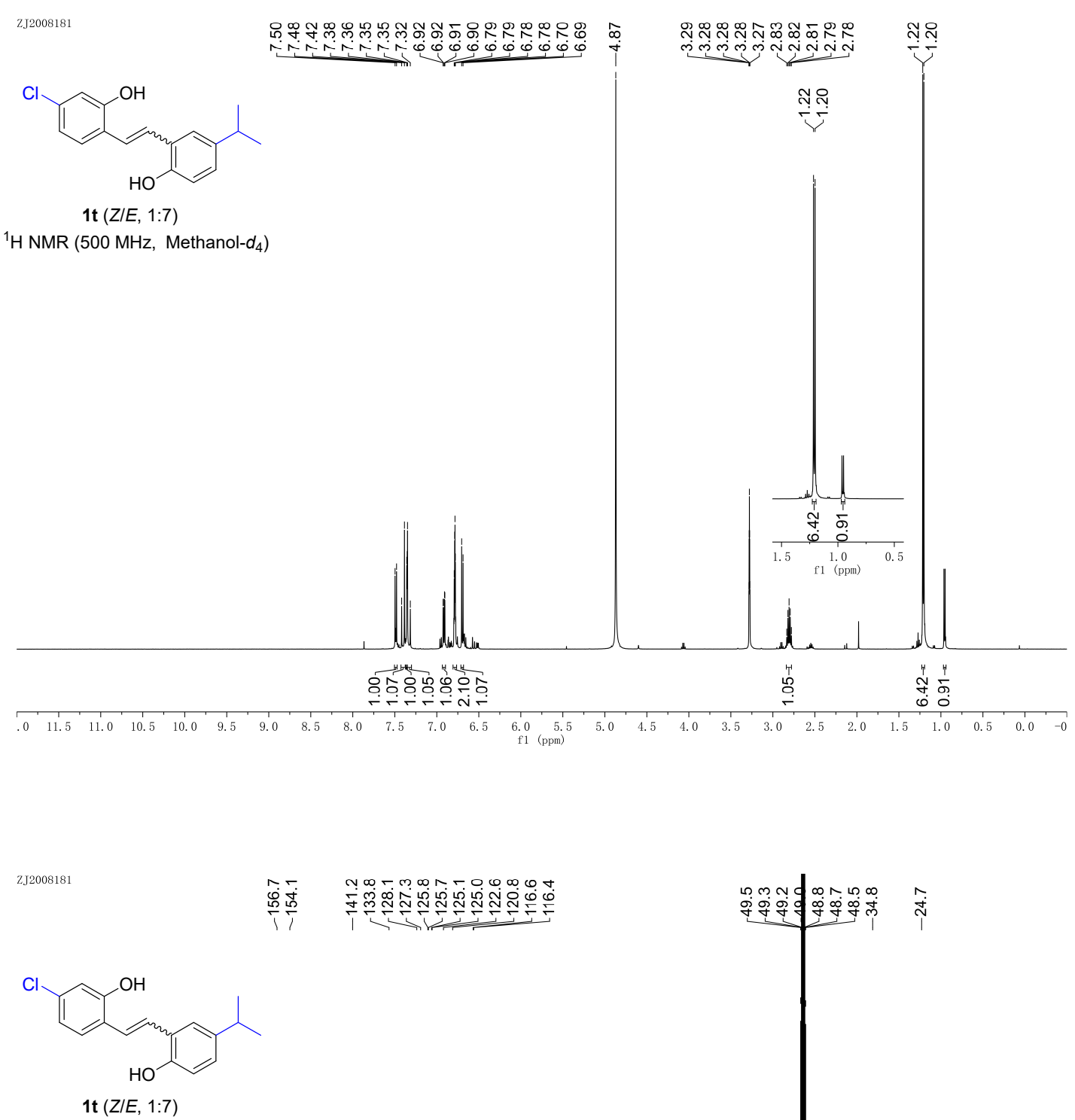

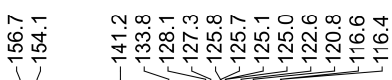

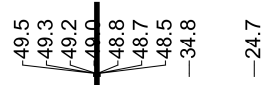

${ }^{1} \mathrm{H}$ NMR $\left(500 \mathrm{MHz}\right.$, Methanol- $\left.d_{4}\right)$

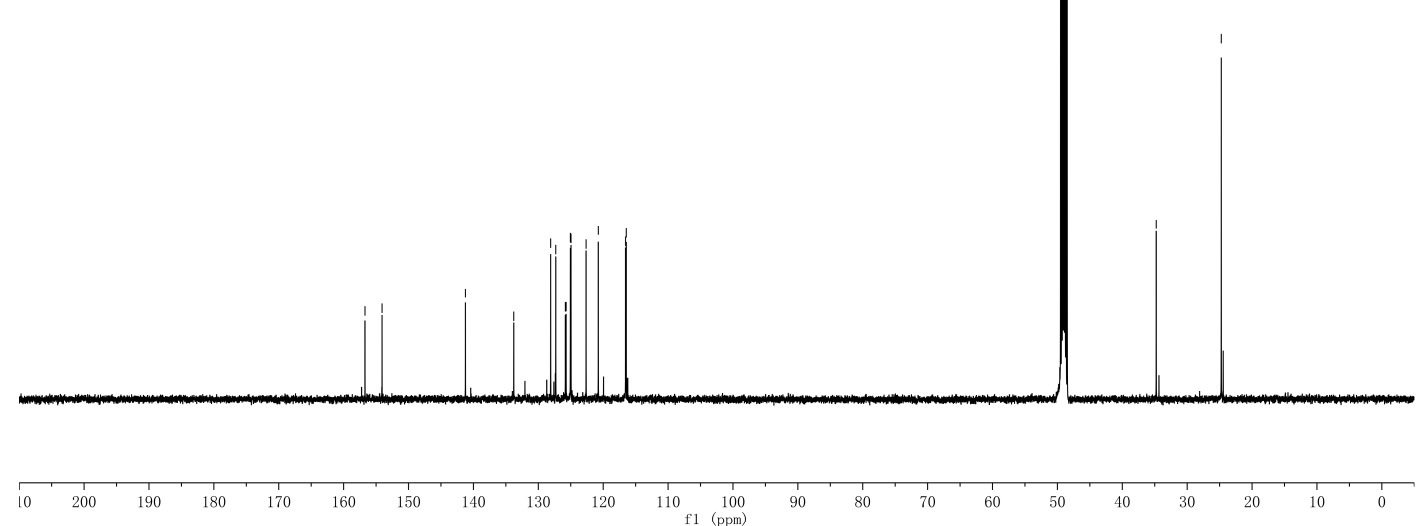




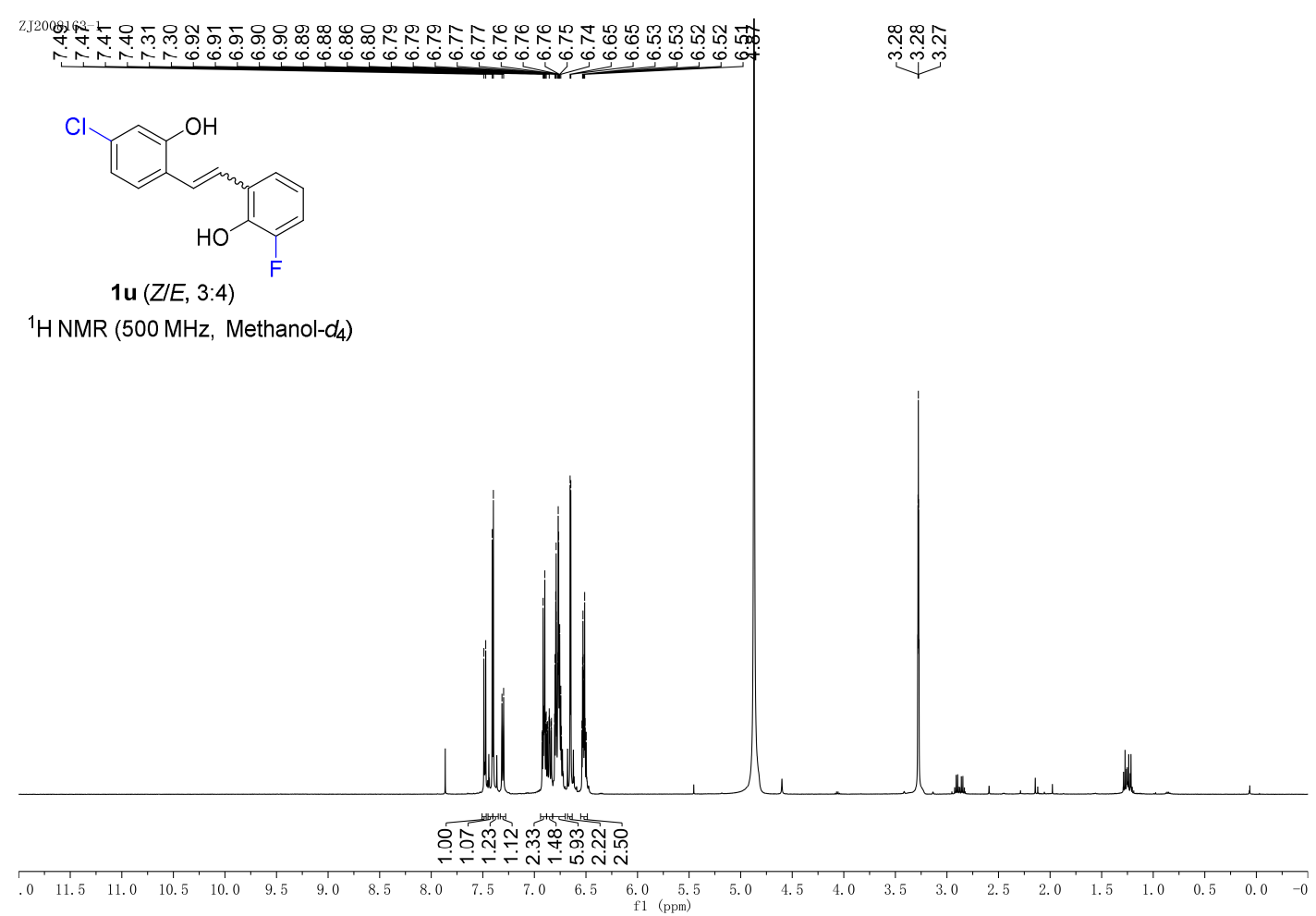

2J2008163-1

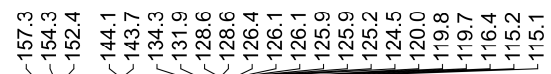

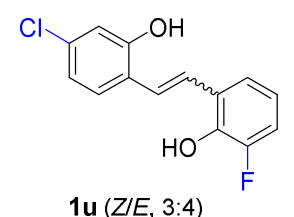

${ }^{1} \mathrm{H}$ NMR $\left(500 \mathrm{MHz}\right.$, Methanol- $\left.d_{4}\right)$

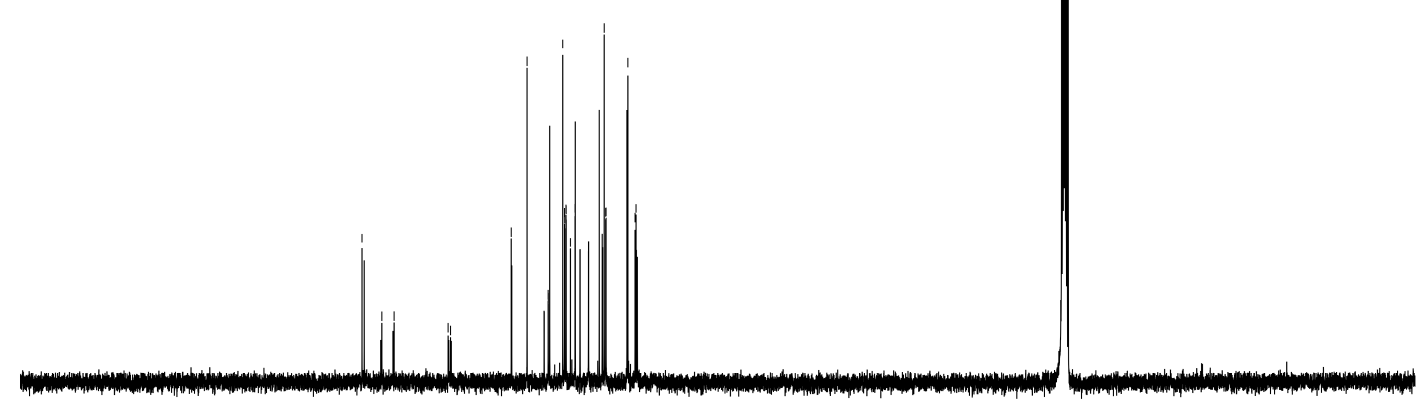

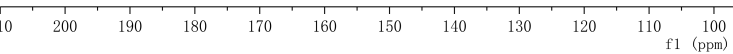


zj2008163F<smiles>Oc1cc(Cl)ccc1/C=C/c1cccc(F)c1O</smiles>

官事

1u (Z/E, 3:4)

${ }^{1} \mathrm{H}$ NMR $\left(500 \mathrm{MHz}\right.$, Methanol- $\left.d_{4}\right)$

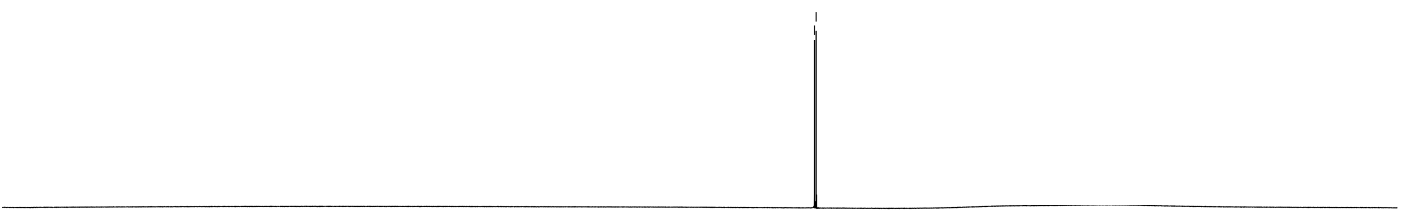

\begin{tabular}{llllllllllllllllllllllllllllllll}
\hline & 0 & -10 & -20 & -30 & -40 & -50 & -60 & -70 & -80 & -90 & -100 & -110 & -120 & -130 & -140 & -150 & -160 & -170 & -180 & -190 & -200 & -210 & -220 & -230 & -2 \\
\hline
\end{tabular} 


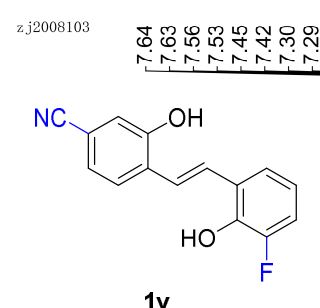

${ }^{1} \mathrm{H}$ NMR $\left(500 \mathrm{MHz}\right.$, Methanol- $\left.d_{4}\right)$
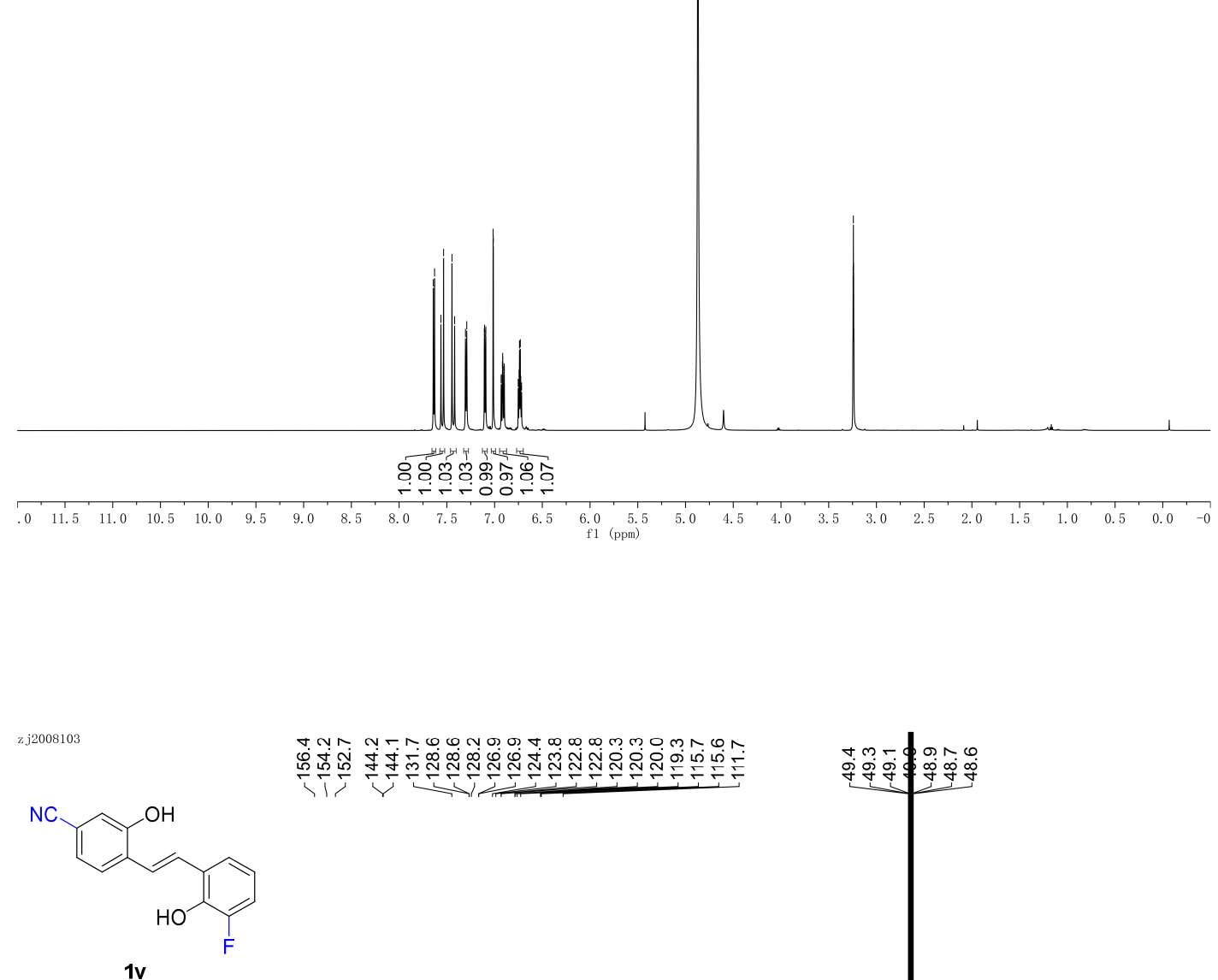

${ }^{13} \mathrm{C}$ NMR $\left(125 \mathrm{MHz}\right.$, Methanol- $\left.d_{4}\right)$

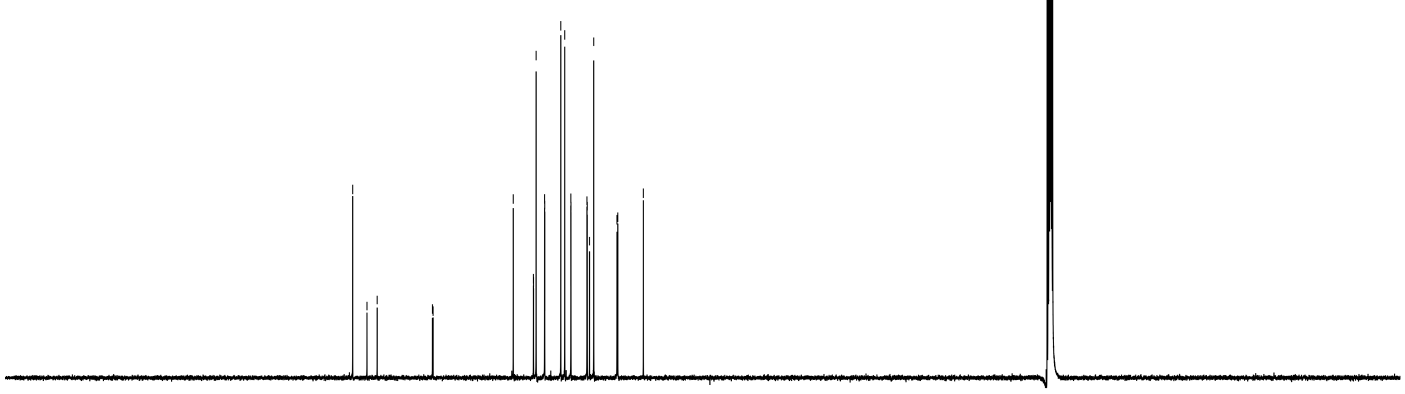

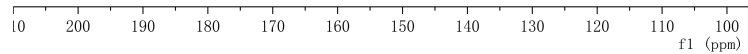




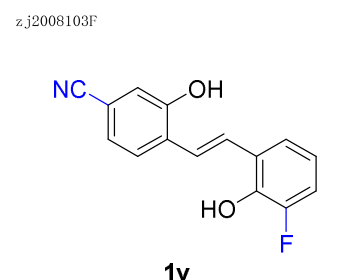

${ }^{19} \mathrm{~F}$ NMR $\left(471 \mathrm{MHz}\right.$, Methanol- $\left.d_{4}\right)$

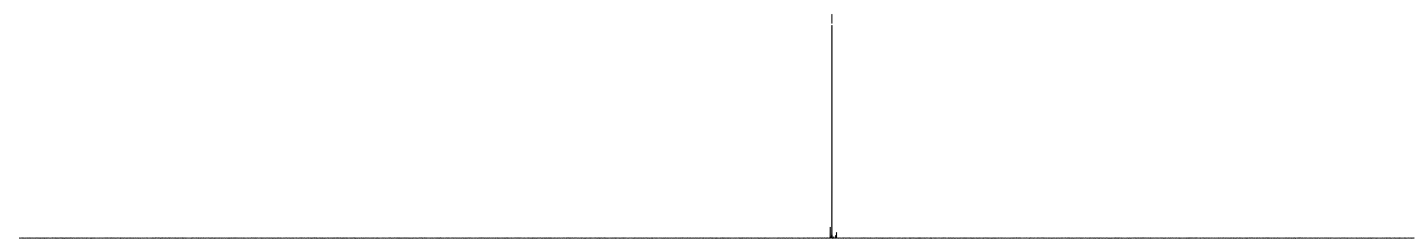

\begin{tabular}{llllllllllllllllllllllllllllllll}
\hline & 0 & -10 & -20 & -30 & -40 & -50 & -60 & -70 & -80 & -90 & -100 & -110 & -120 & -130 & -140 & -150 & -160 & -170 & -180 & -190 & -200 & -210 & -220 & -230 & -2 \\
\hline
\end{tabular} 

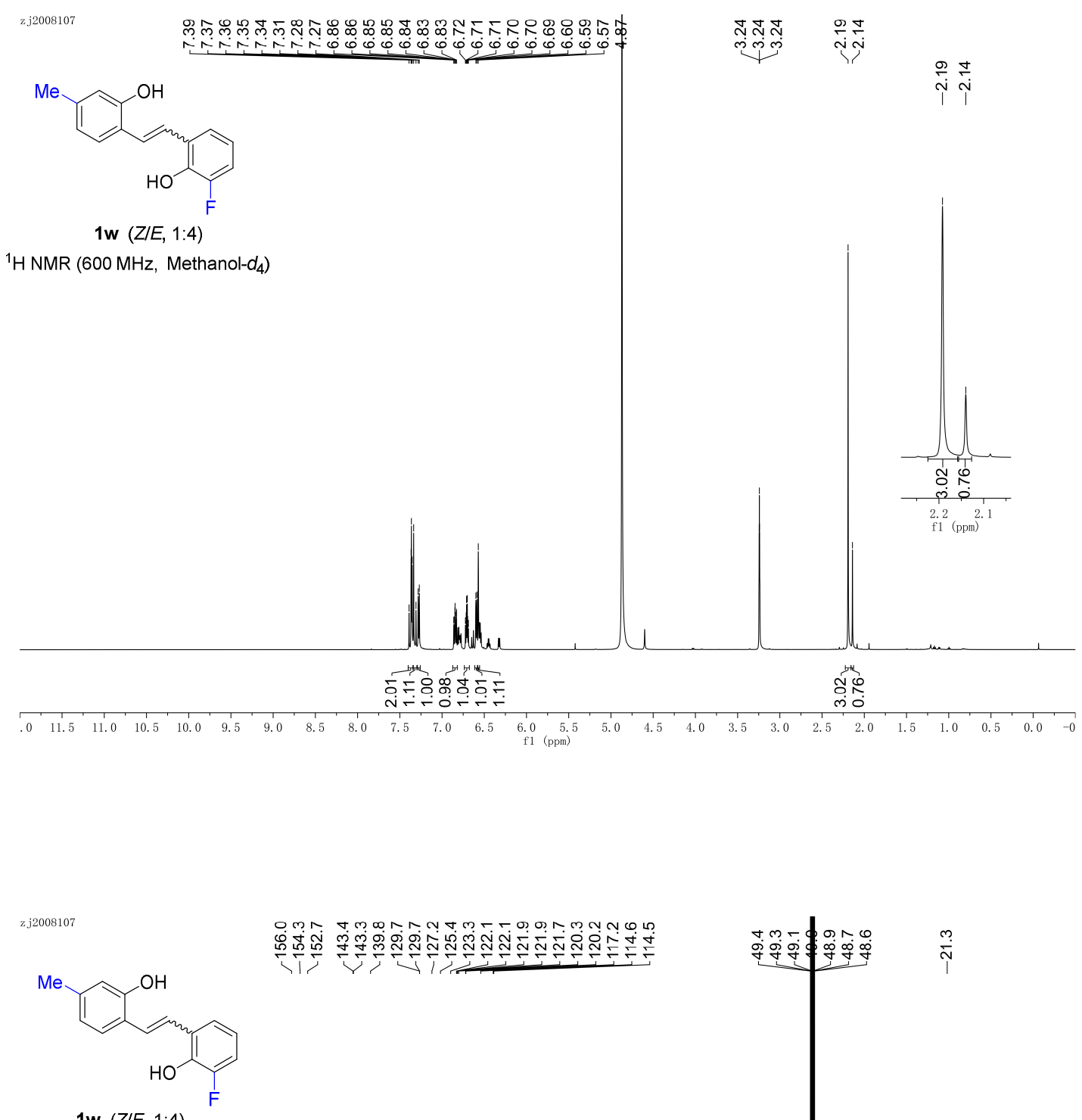

${ }^{13} \mathrm{C}$ NMR (150 MHz, Methanol-d $\left.d_{4}\right)$

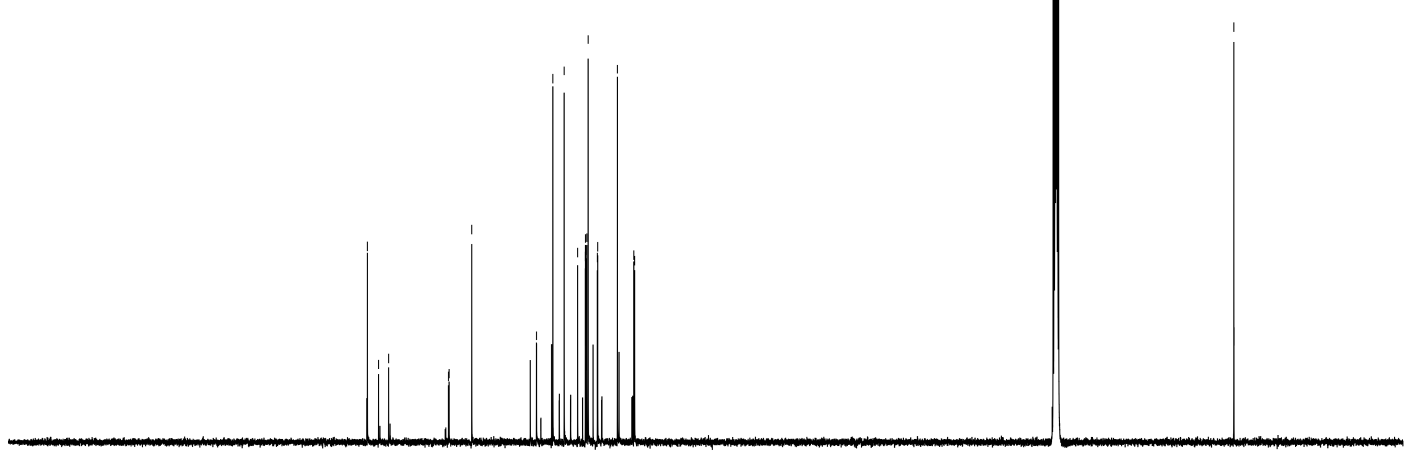

\begin{tabular}{l}
$T 210$ \\
\hline
\end{tabular} 
zj2008107F

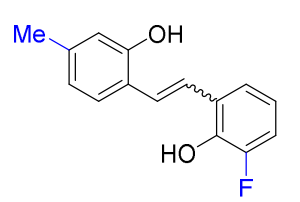

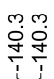

$1 w$ (ZIE, 1:4)

${ }^{19} \mathrm{~F}$ NMR $\left(471 \mathrm{MHz}\right.$, Methanol- $\left.d_{4}\right)$

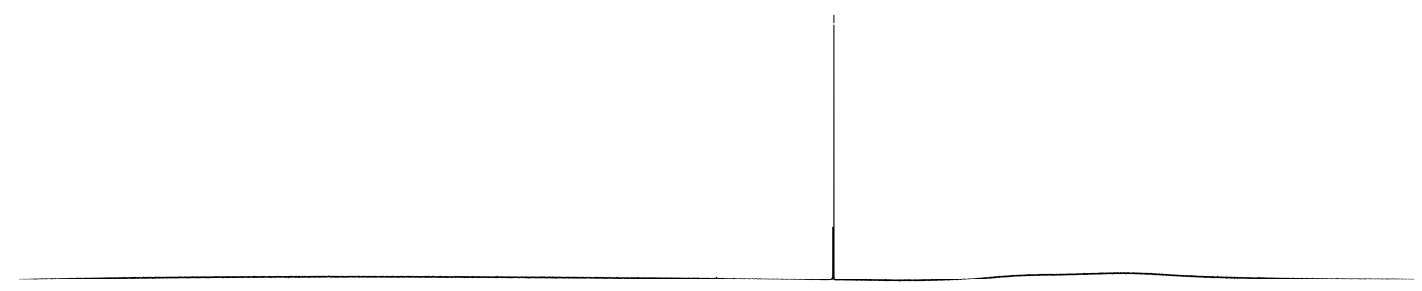

\begin{tabular}{lllllllllllllllllllllllllllllll}
\hline & $T_{0}$ & -10 & -20 & -30 & -40 & -50 & -60 & -70 & -80 & -90 & -100 & -110 & -120 & -130 & -140 & -150 & -160 & -170 & -180 & -190 & -200 & -210 & -220 & -230 & -2 \\
\hline
\end{tabular} 


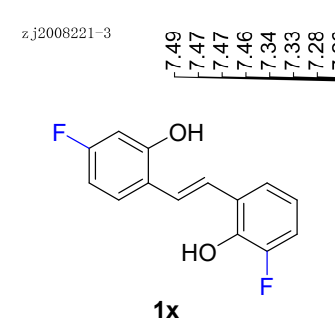

${ }^{1} \mathrm{H}$ NMR $\left(600 \mathrm{MHz}\right.$, Methanol- $\left.d_{4}\right)$

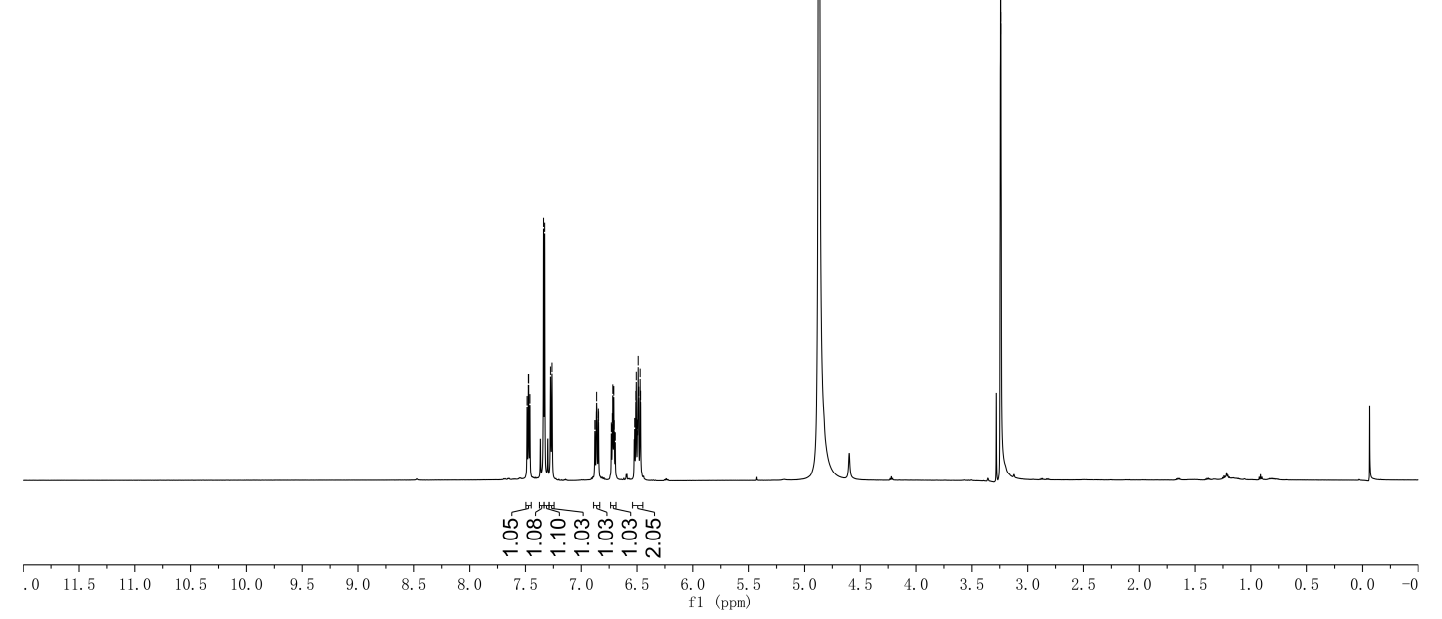

zj2008221-3

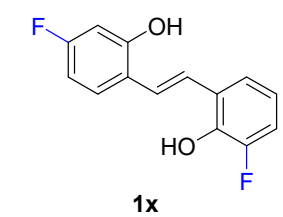

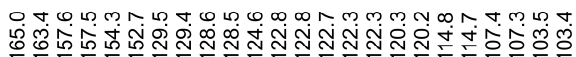

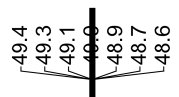

${ }^{13} \mathrm{C}$ NMR $\left(150 \mathrm{MHz}\right.$, Methanol- $\left.d_{4}\right)$

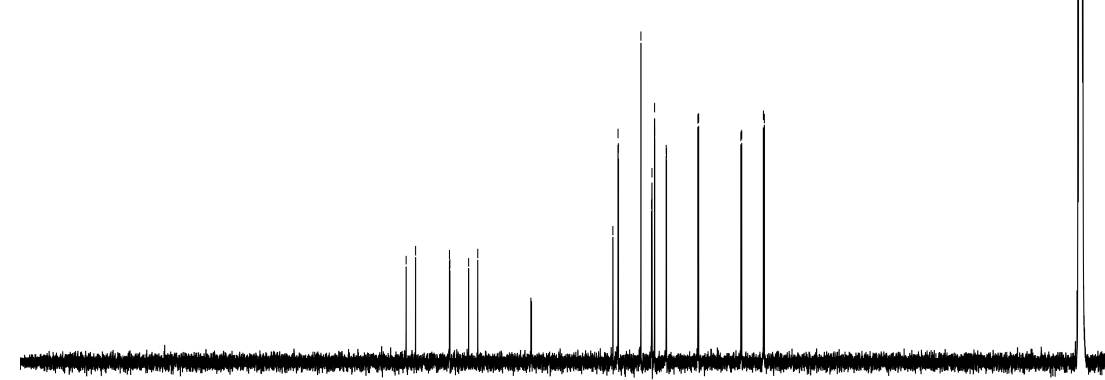

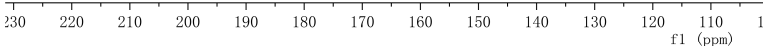




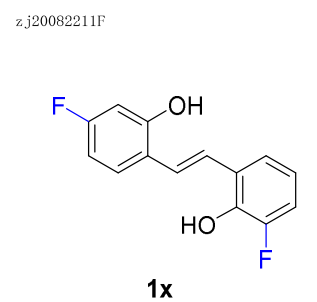

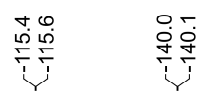

${ }^{19} \mathrm{~F}$ NMR $\left(471 \mathrm{MHz}\right.$, Methanol- $\left.d_{4}\right)$

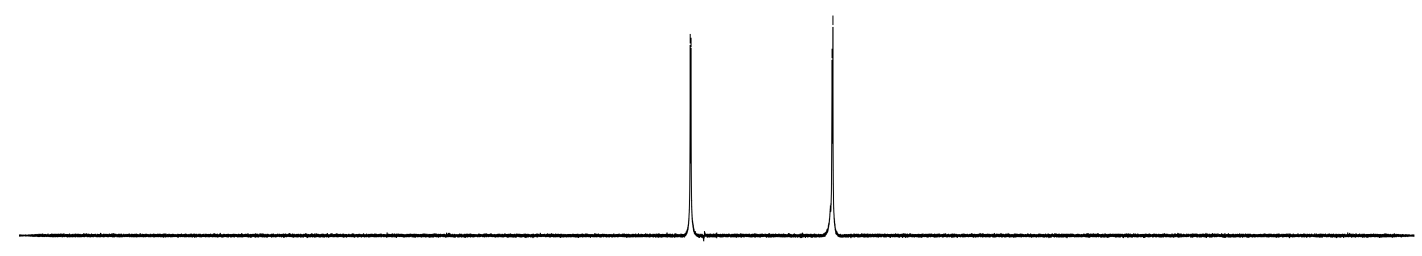

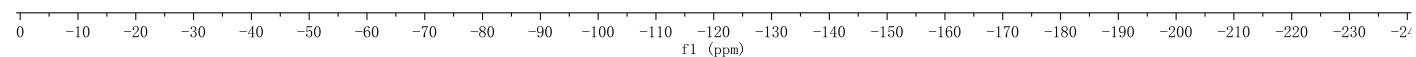




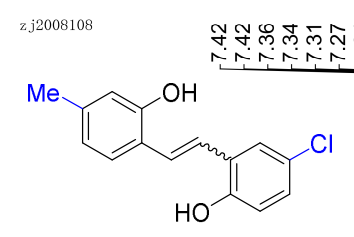

$1 y$ (ZIE, 2:1)

${ }^{1} \mathrm{H}$ NMR $\left(600 \mathrm{MHz}\right.$, Methanol- $\left.d_{4}\right)$
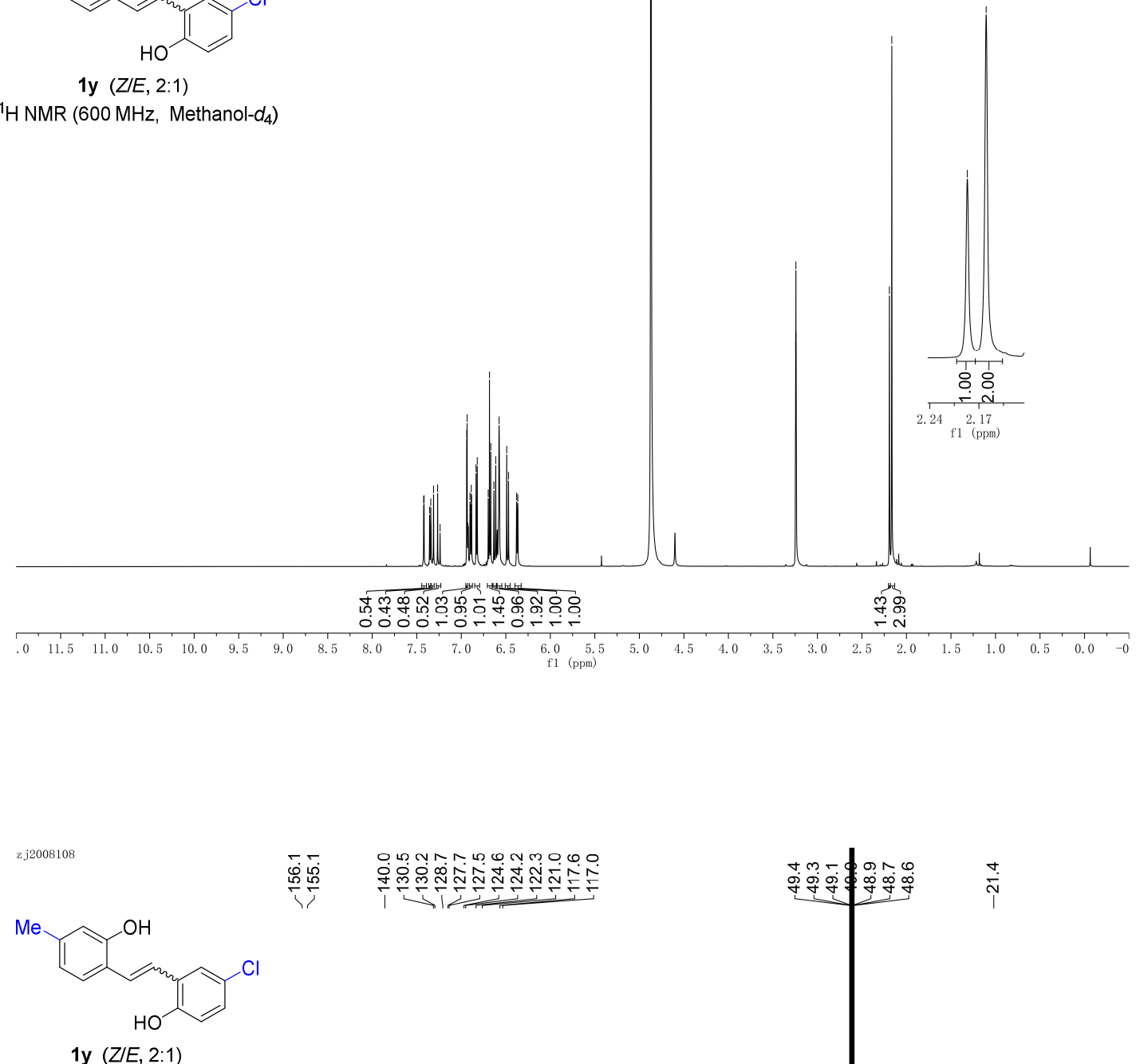

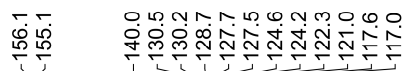

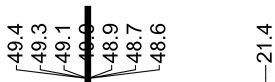

${ }^{13} \mathrm{C}$ NMR (150 MHz, Methanol- $\left.d_{4}\right)$

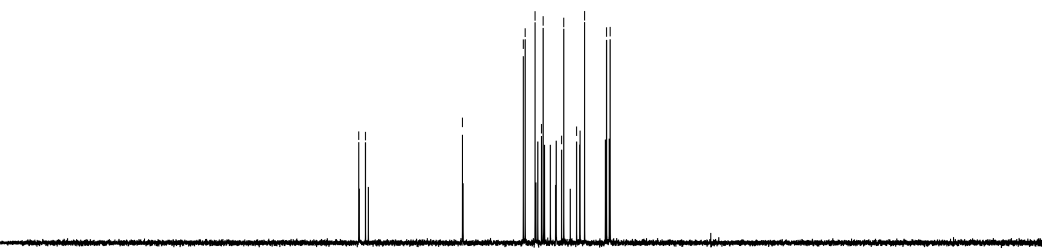

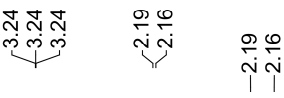




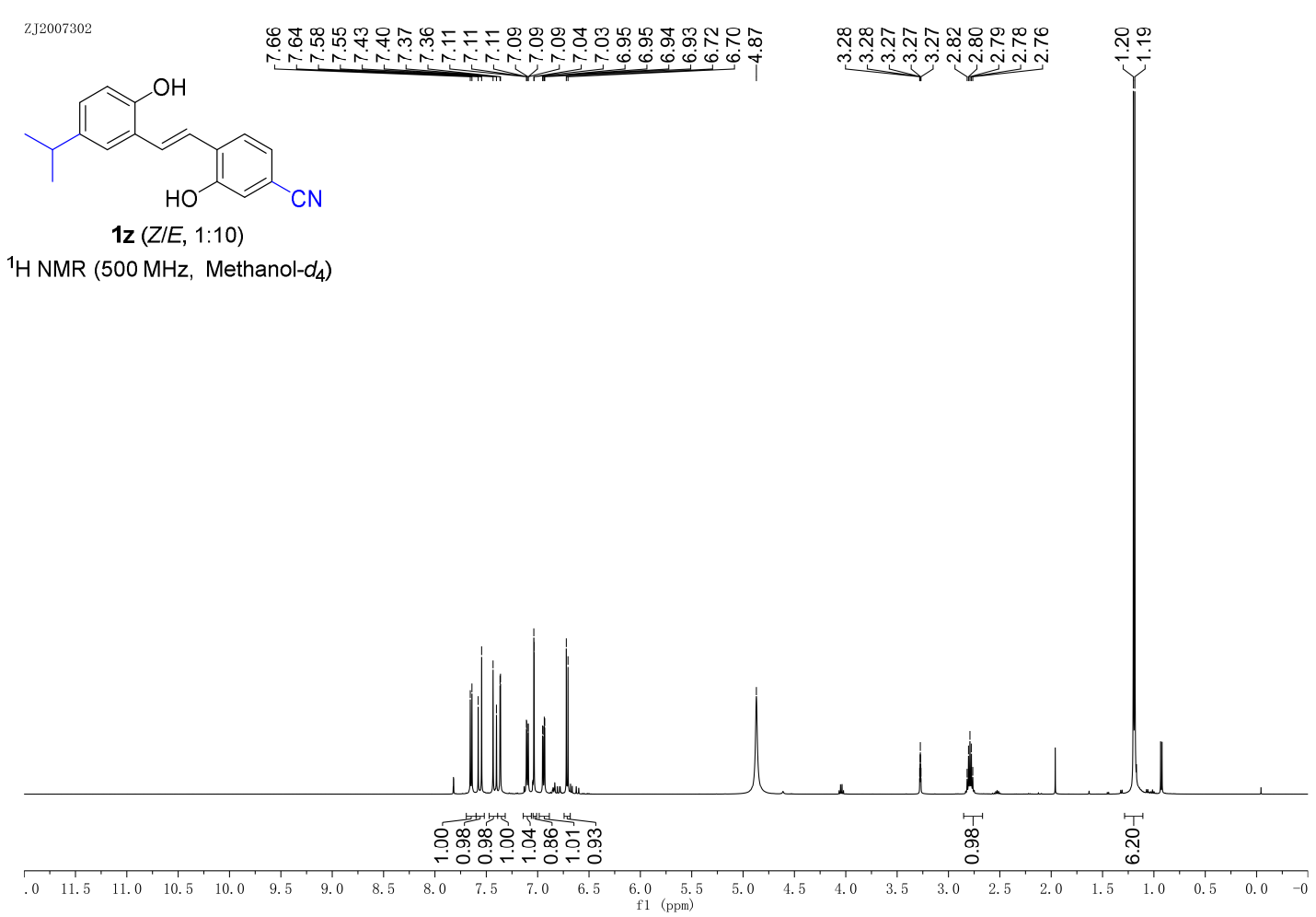

2J2007302

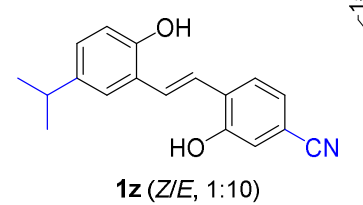

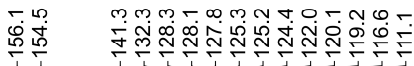

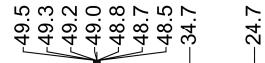

${ }^{13} \mathrm{C}$ NMR (125 MHz, Methanol- $\left.d_{4}\right)$

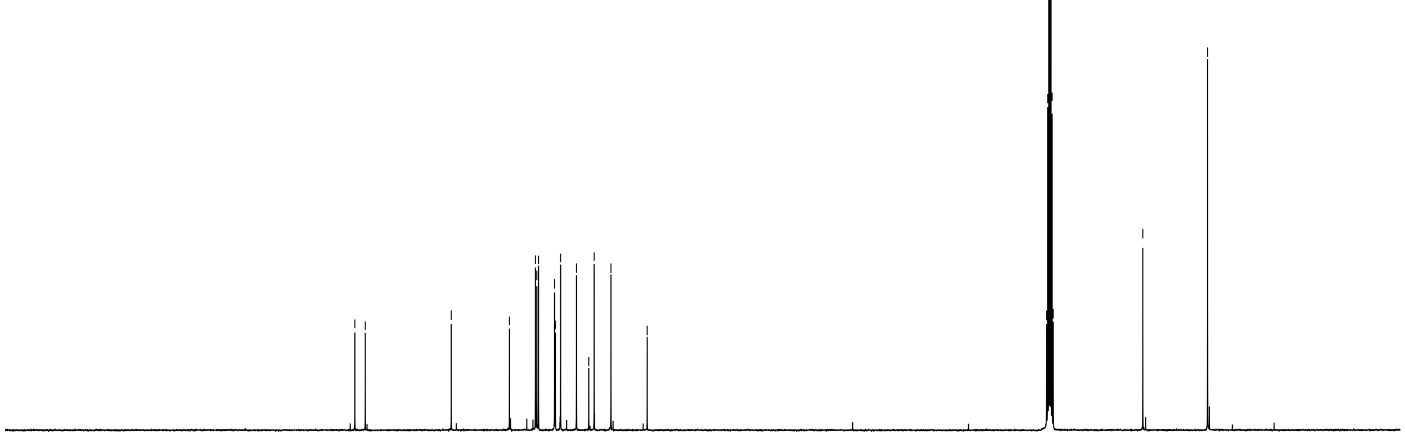

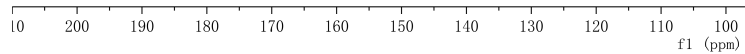




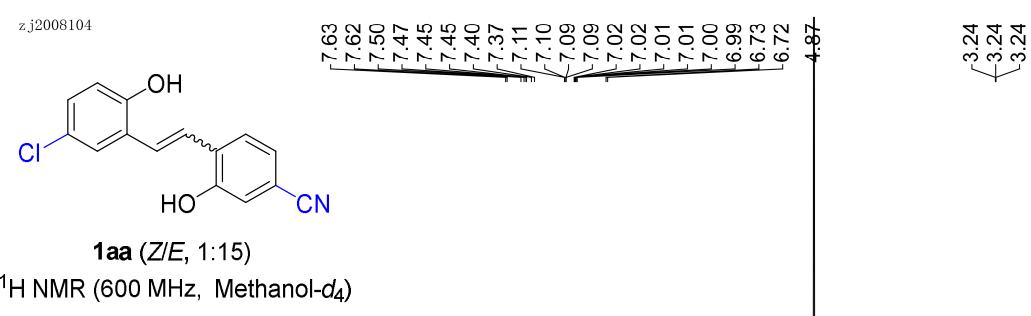

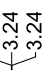

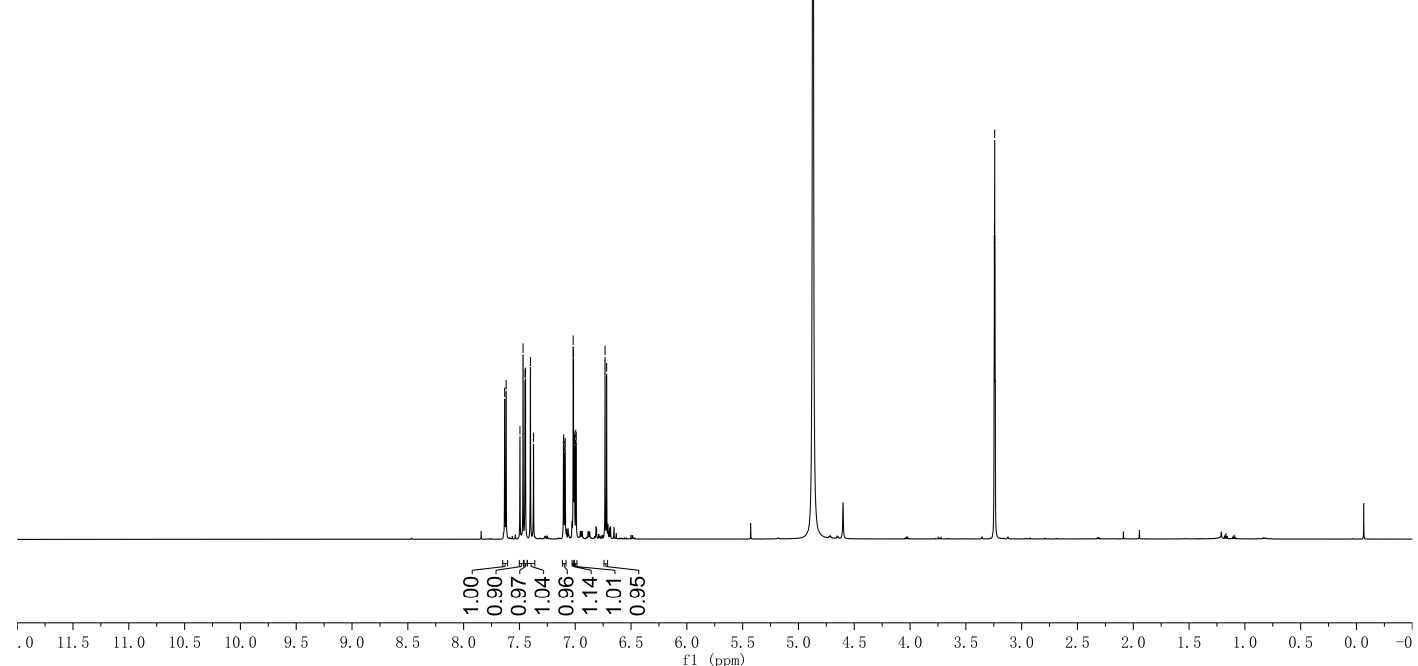

zj200810

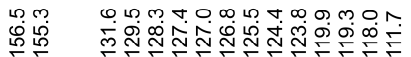<smiles>N#Cc1ccc(/C=C/c2cc(Cl)ccc2O)c(O)c1</smiles>

1aa (ZIE, 1:15)

${ }^{13} \mathrm{C}$ NMR $\left(150 \mathrm{MHz}\right.$, Methanol- $\left.d_{4}\right)$
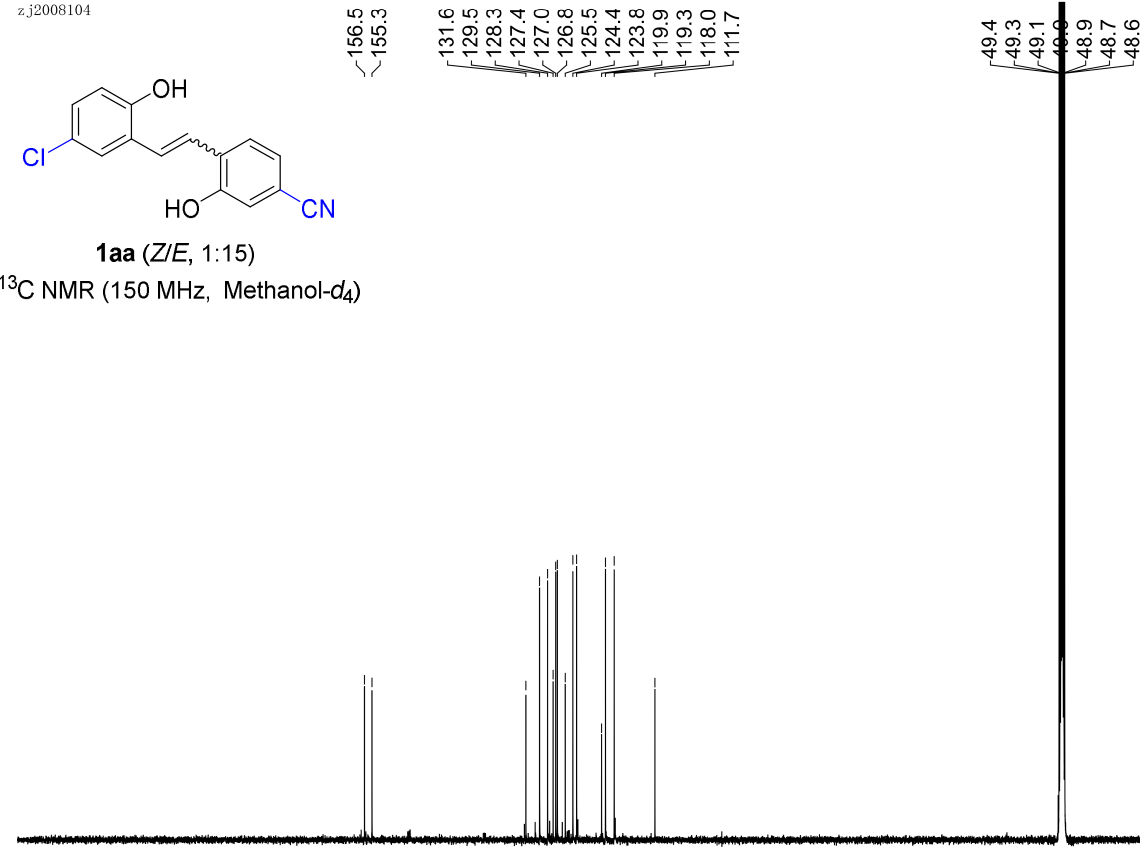

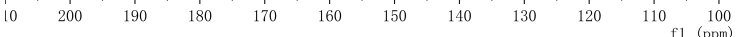



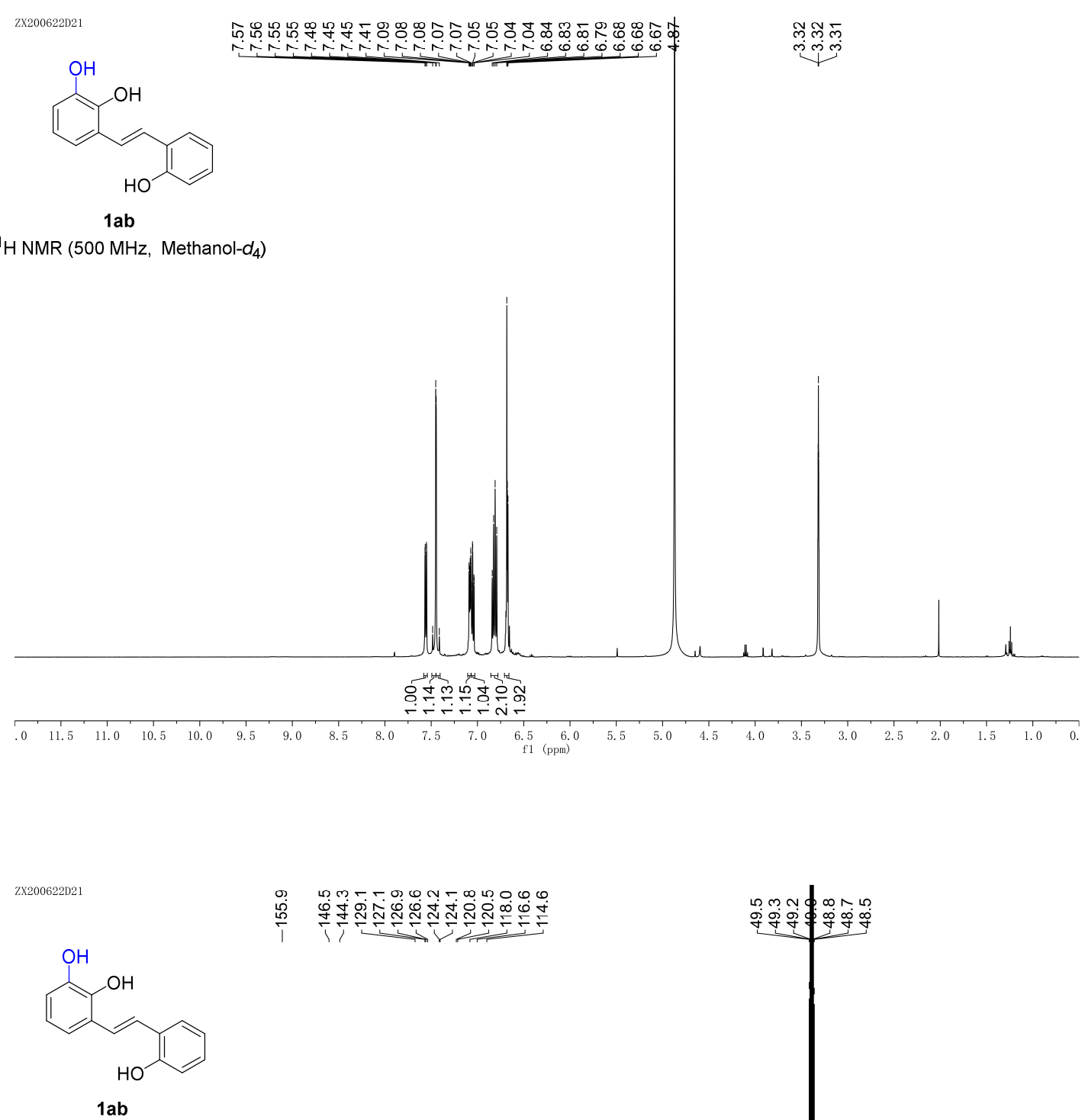

${ }^{13} \mathrm{C}$ NMR $\left(125 \mathrm{MHz}\right.$, Methanol- $\left.d_{4}\right)$

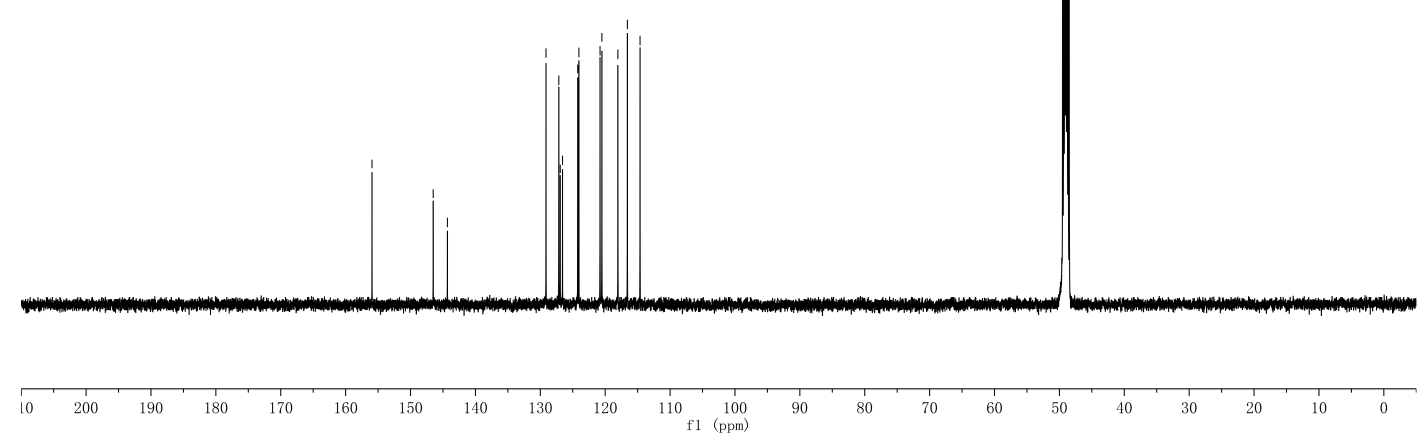




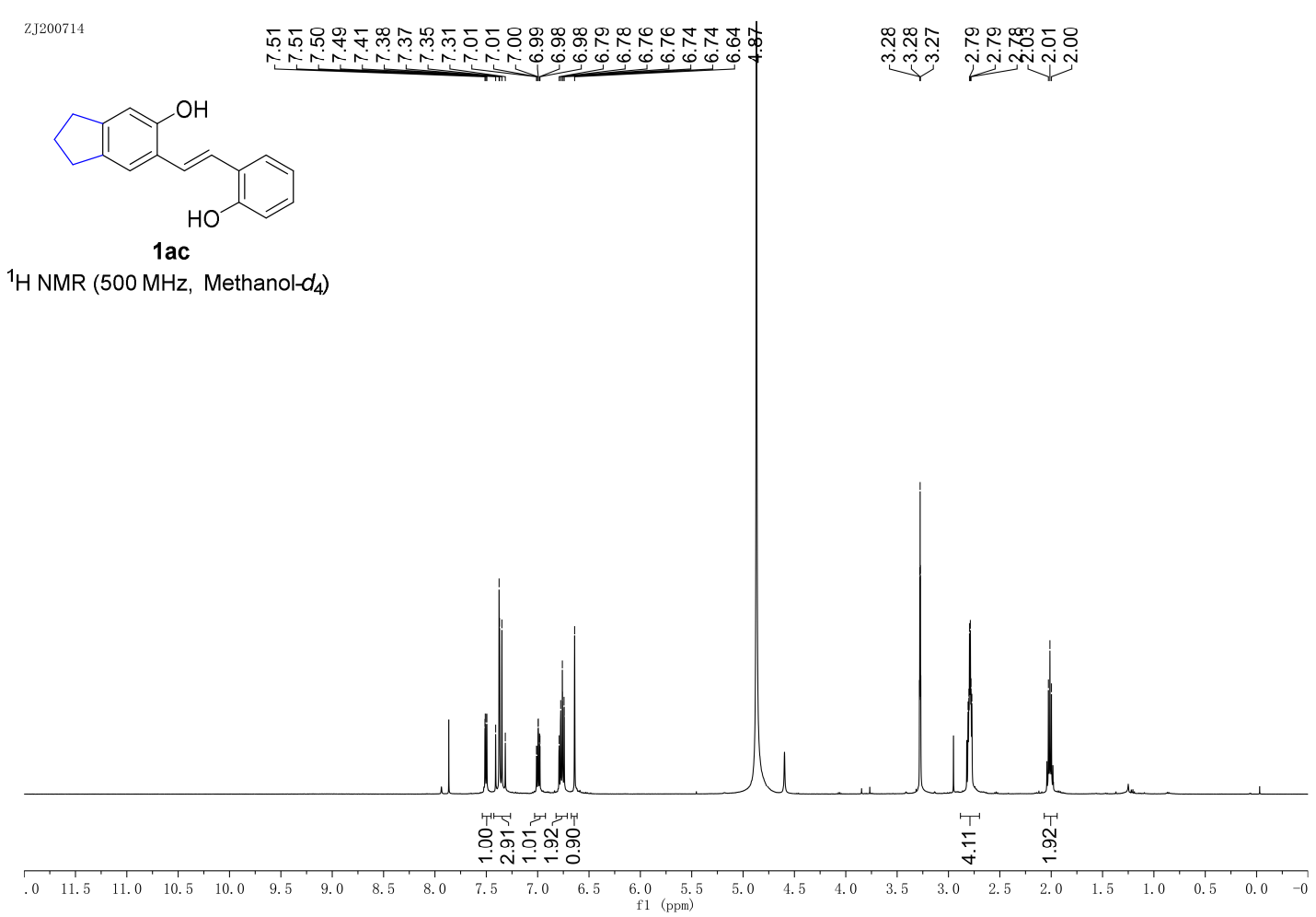

ZJ200714
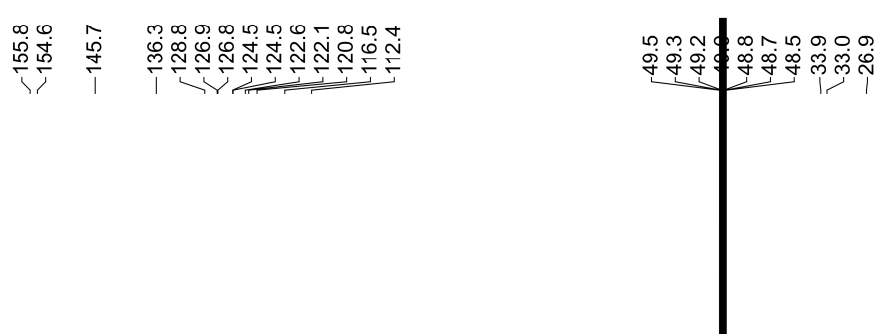

${ }^{13} \mathrm{C}$ NMR $\left(125 \mathrm{MHz}\right.$, Methanol- $\left.d_{4}\right)$
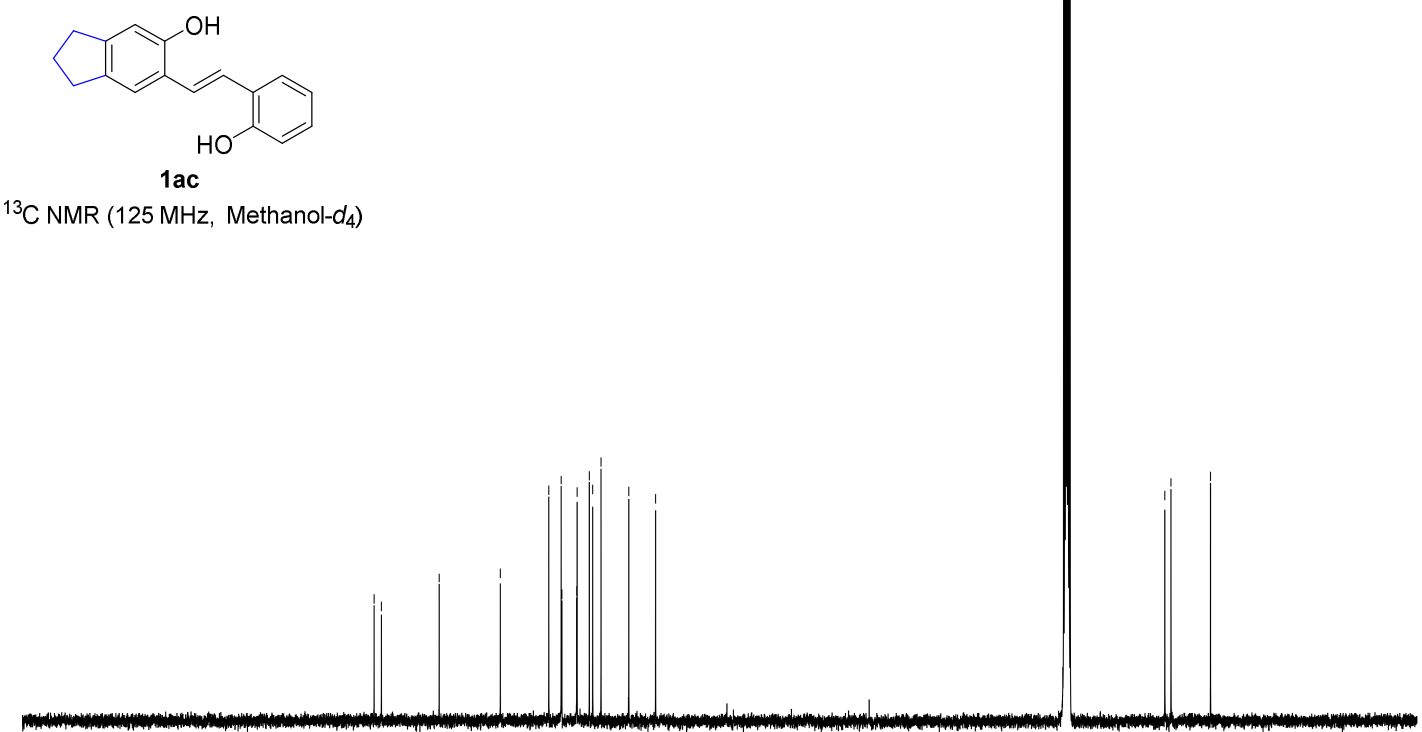

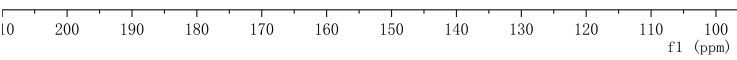




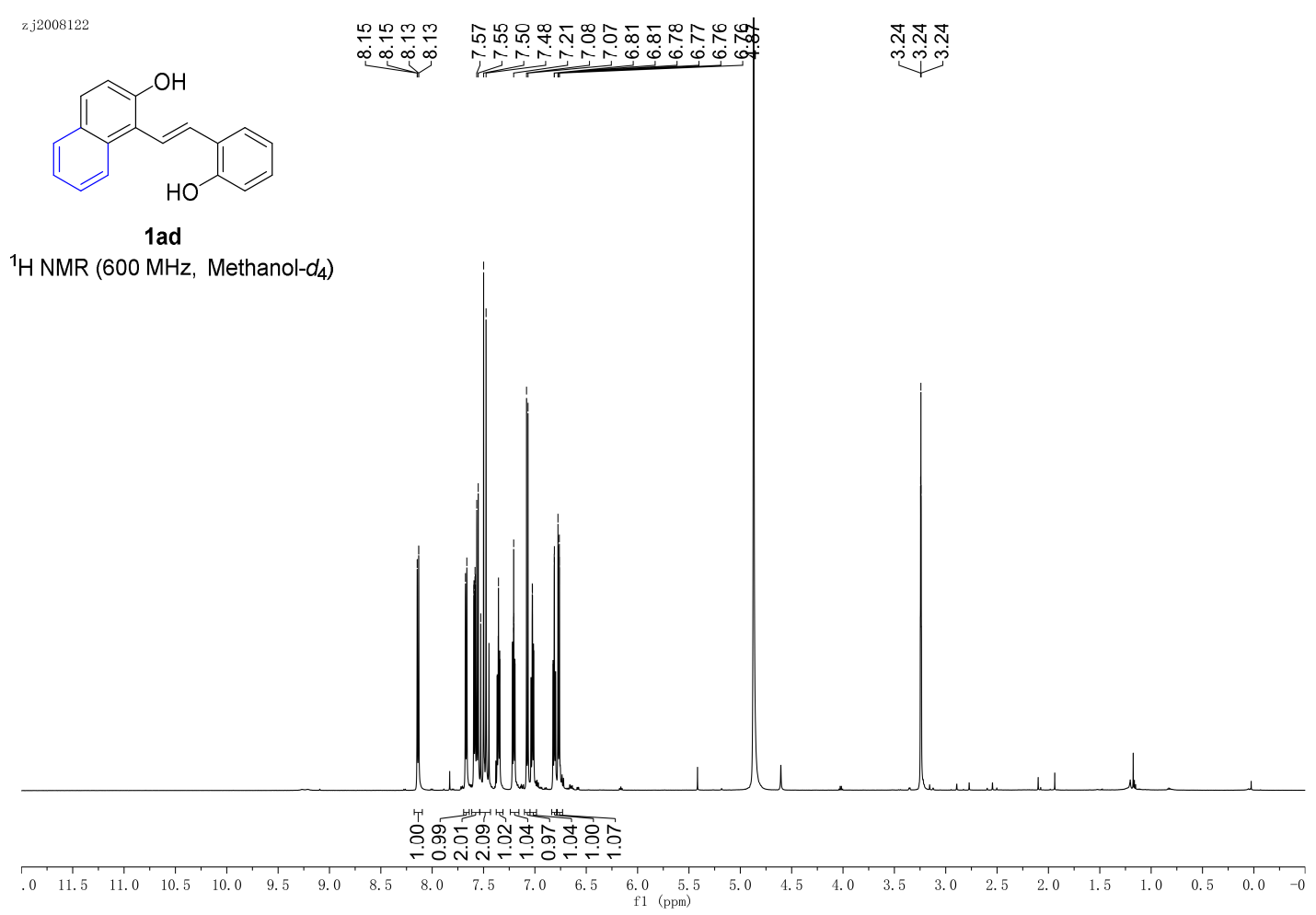

zj2008122

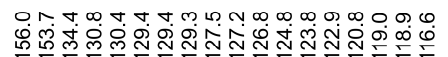

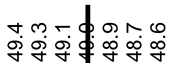<smiles>Oc1ccccc1/C=C/c1cccc2ccccc12</smiles>

$1 \mathrm{ad}$

${ }^{13} \mathrm{C}$ NMR $\left(150 \mathrm{MHz}\right.$, Methanol- $\left.d_{4}\right)$

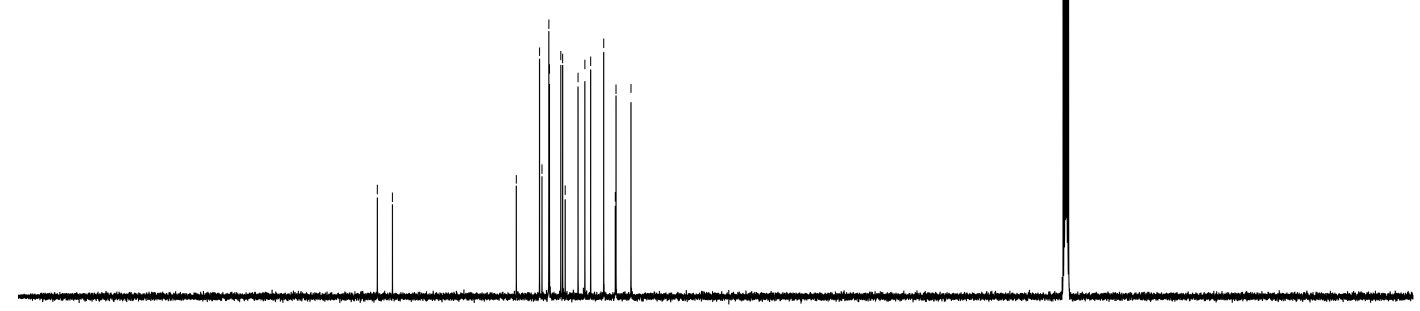

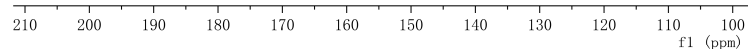




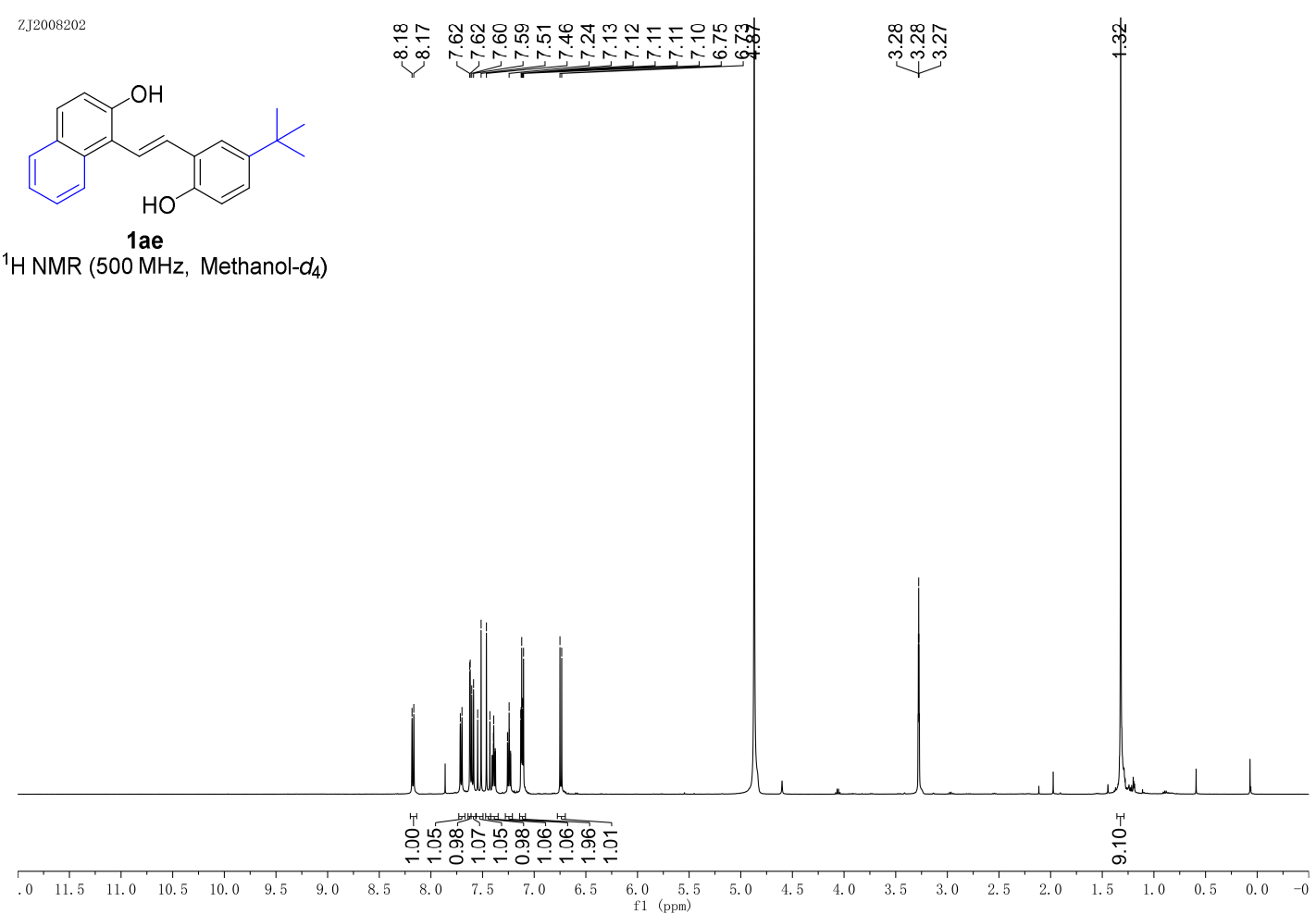

ZJ 2008202
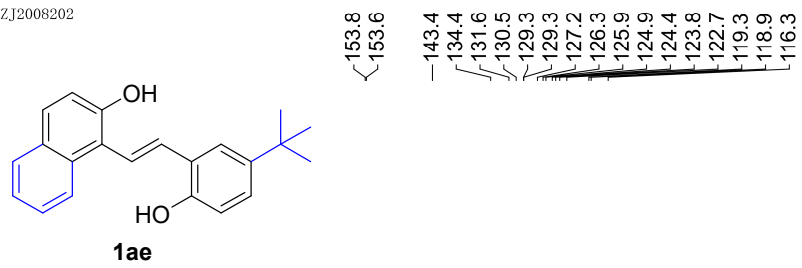

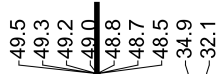

${ }^{13} \mathrm{C}$ NMR $\left(125 \mathrm{MHz}\right.$, Methanol- $\left.d_{4}\right)$

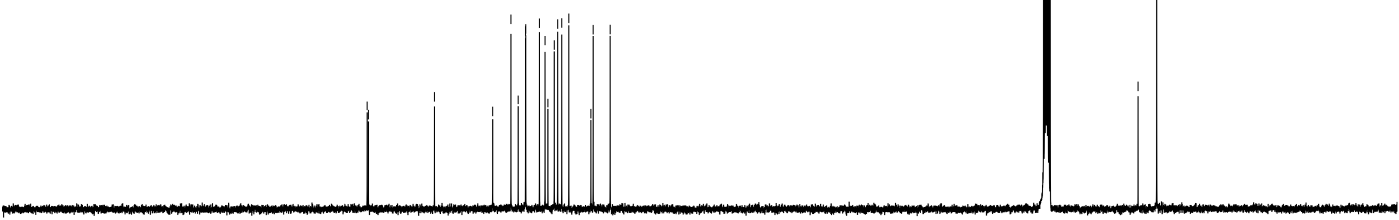

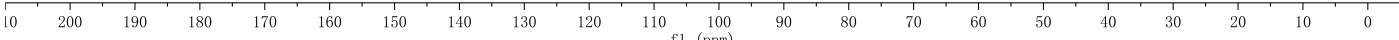




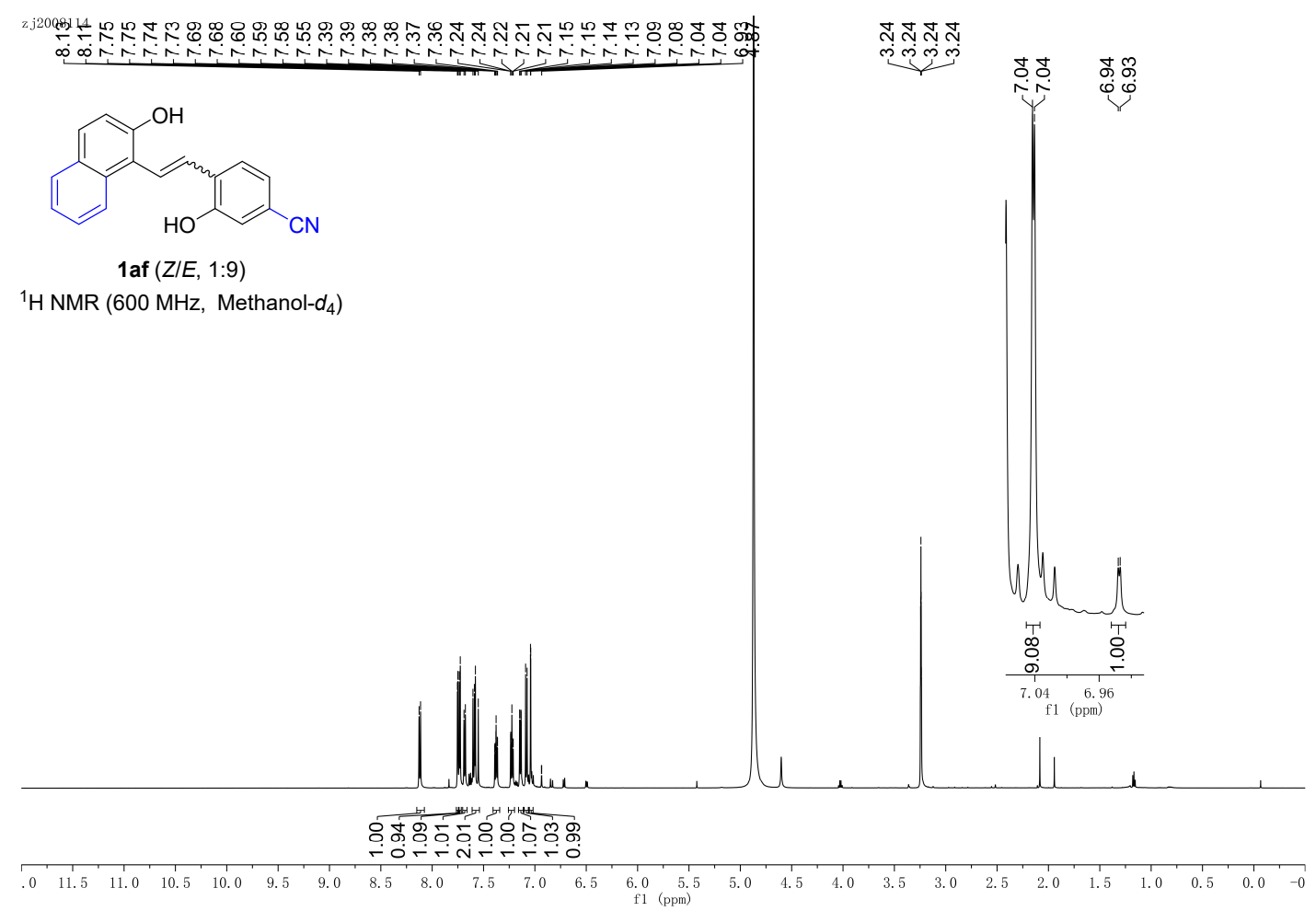

zj2008114
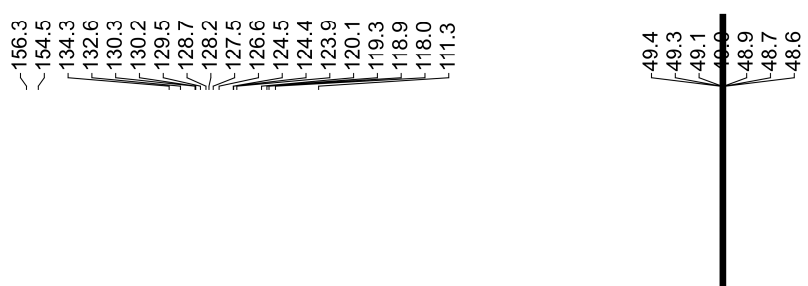

1af (ZIE, 1:9)

${ }^{13} \mathrm{C} \mathrm{NMR} \mathrm{(150} \mathrm{MHz}$, Methanol- $\left.d_{4}\right)$

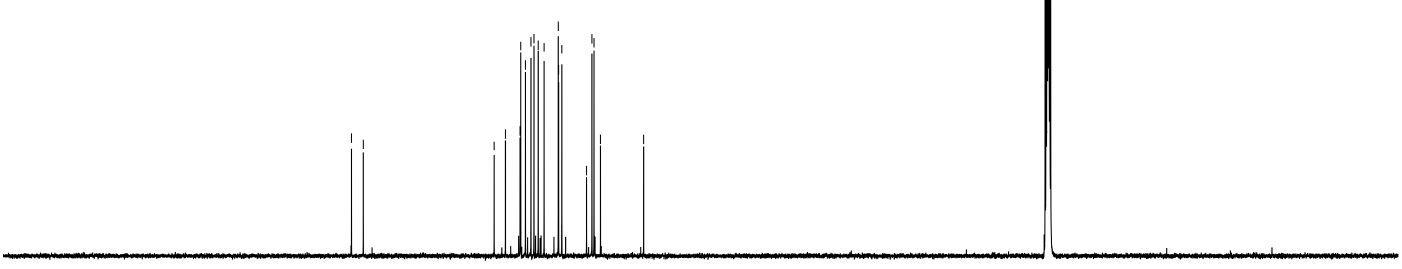

$\begin{array}{lllllllllllll}10 & 200 & 190 & 180 & 170 & 160 & 150 & 140 & 130 & 120 & 110 & 1 \\ \mathrm{f} 1(\mathrm{pmm})\end{array}$ 

zJ200811

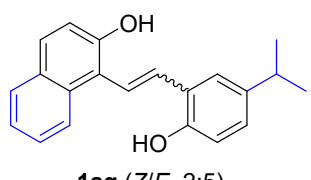

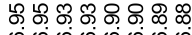

ن

1 ag (Z/E, 2:5)

${ }^{1} \mathrm{H}$ NMR $\left(500 \mathrm{MHz}\right.$, Methanol- $\left.d_{4}\right)$

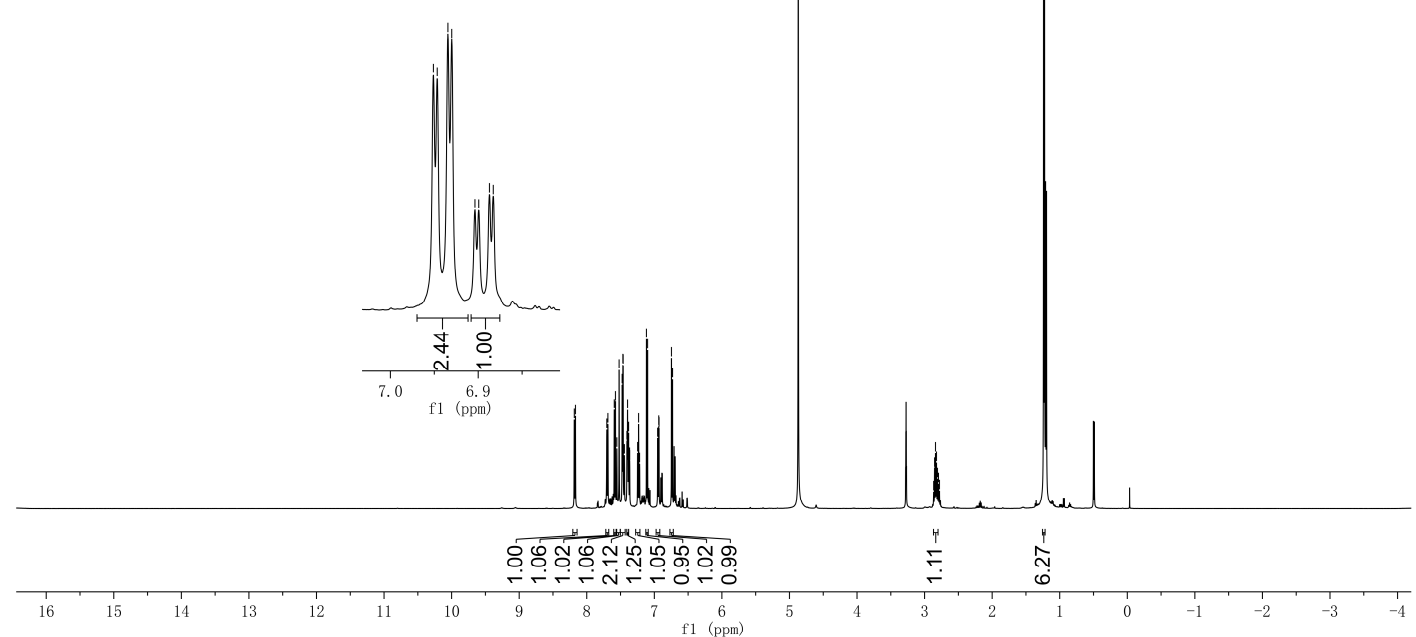

J J200811

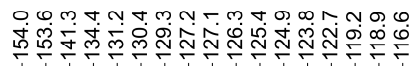

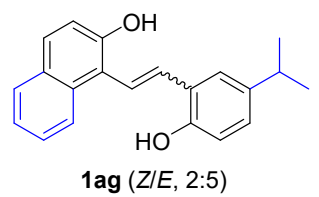

${ }^{13} \mathrm{C}$ NMR $\left(125 \mathrm{MHz}\right.$, Methanol- $\left.d_{4}\right)$
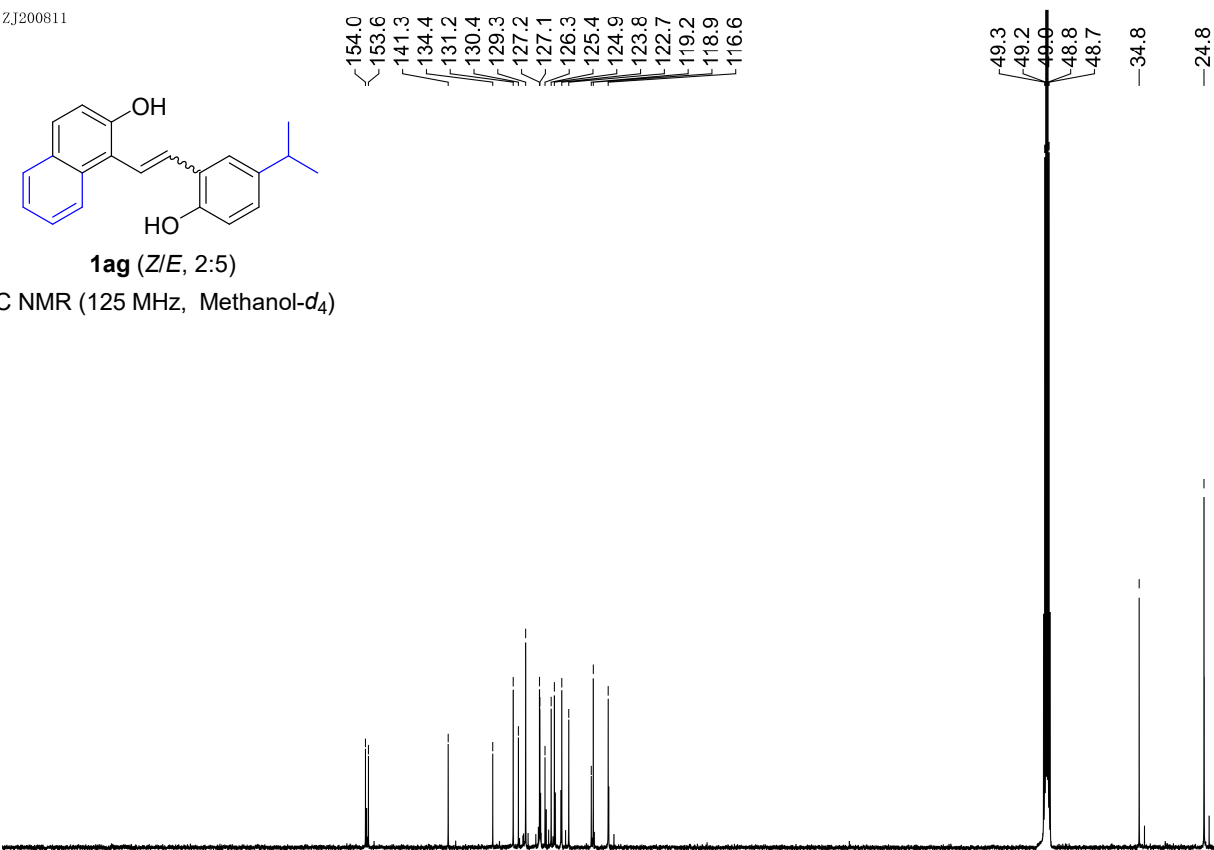

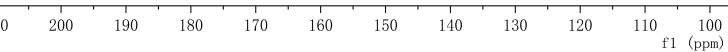



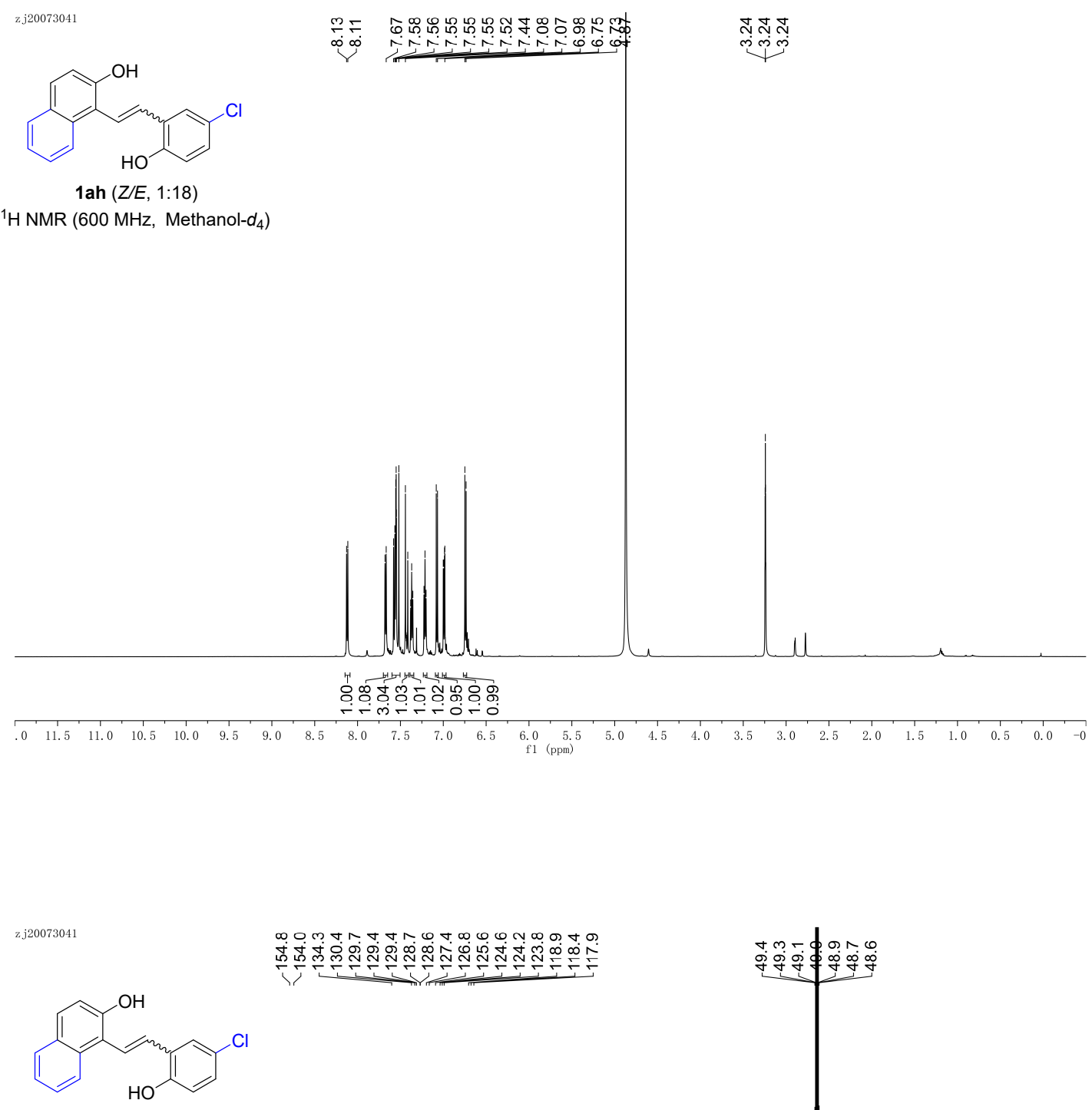

1ah (Z/E, 1:18)

${ }^{13} \mathrm{C}$ NMR $\left(150 \mathrm{MHz}\right.$, Methanol- $\left.d_{4}\right)$

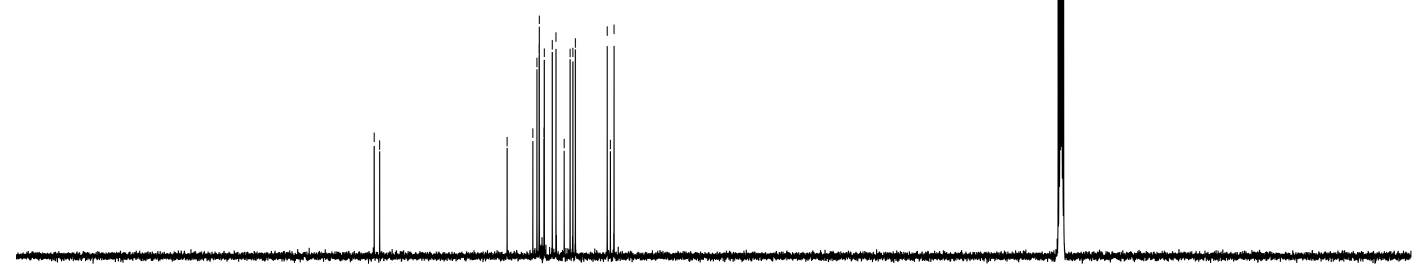

$\begin{array}{llllllllllll}10 & 200 & 190 & 180 & 170 & 160 & 150 & 140 & 130 & 120 & 110 & 1 \\ \mathrm{f} 1(\mathrm{pmm}) & 1\end{array}$ 


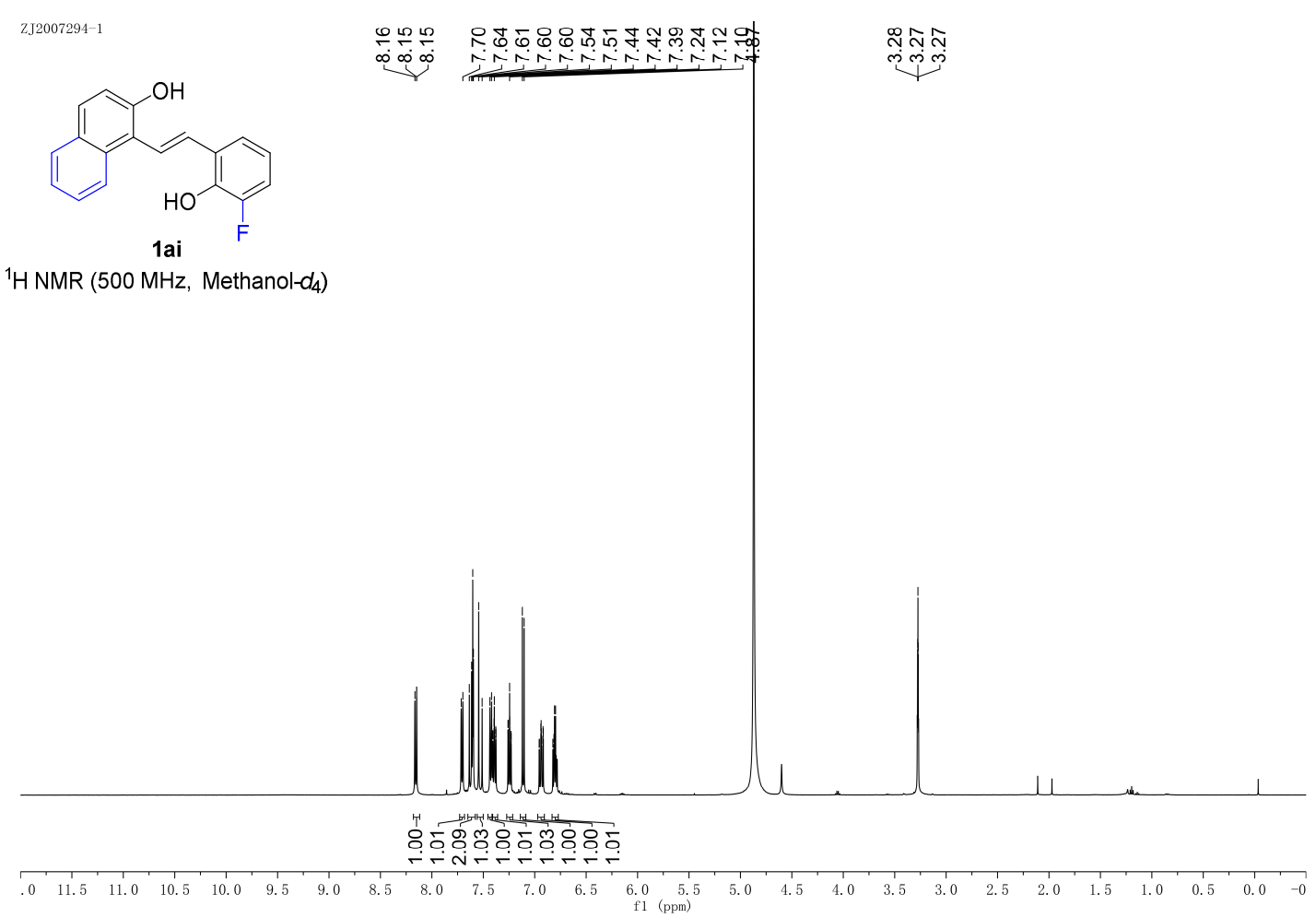

ZJ2007294-1

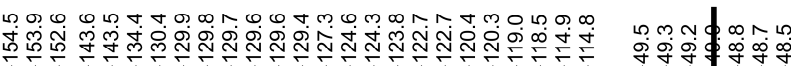

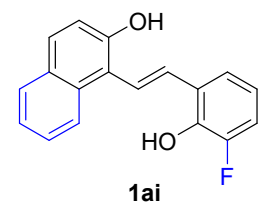

${ }^{13} \mathrm{C}$ NMR $\left(125 \mathrm{MHz}\right.$, Methanol- $d_{4}$ )

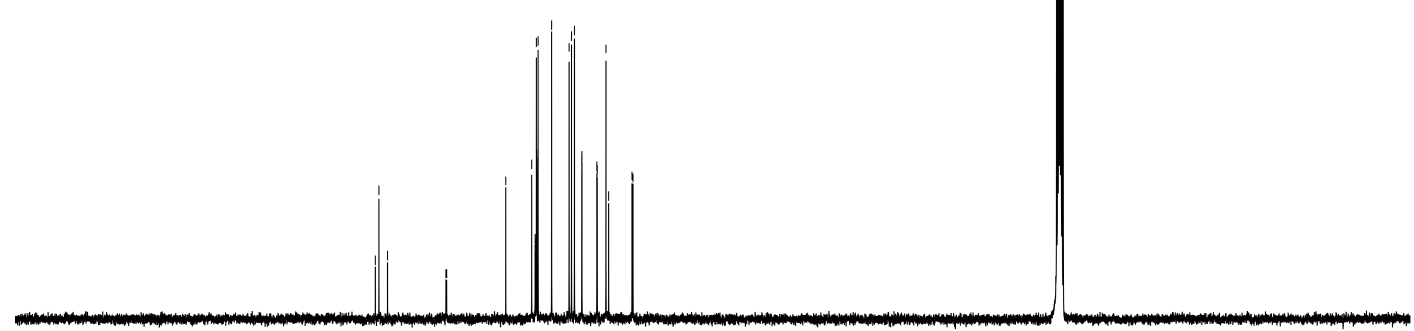

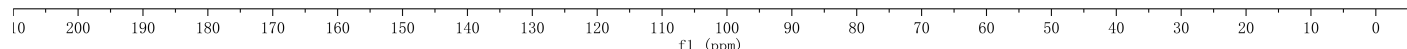


zj2007294F

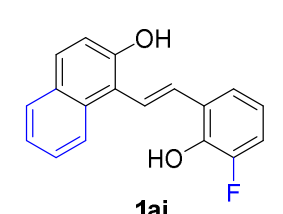

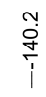

${ }^{19} \mathrm{~F} \mathrm{NMR}\left(471 \mathrm{MHz}\right.$, Methanol-d $\left.d_{4}\right)$

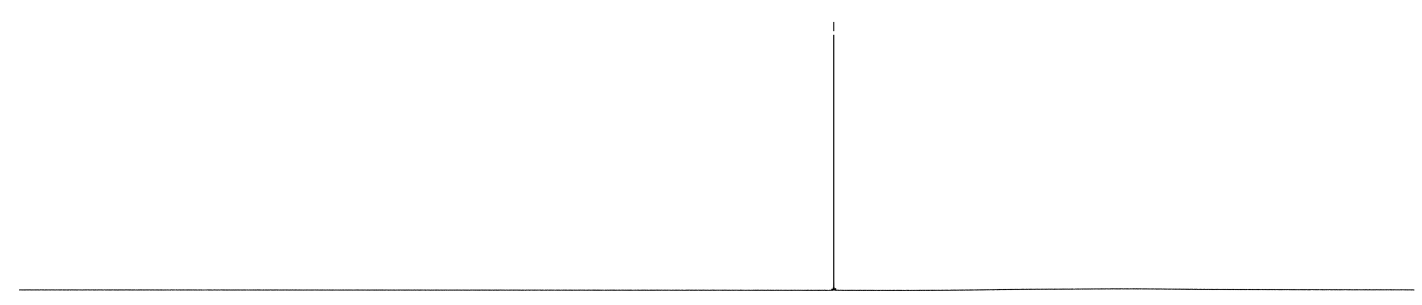

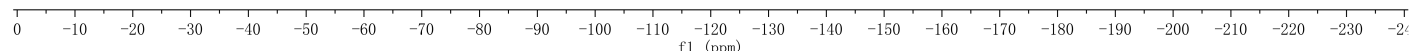


s001192-1

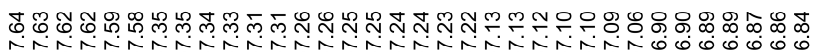

$\stackrel{\substack{\text { i } \\ \text { i }}}{2}$<smiles>Oc1ccccc1/C=C/c1ccccc1N[Sb]</smiles>

1aj (Z/E, 1:15)

${ }^{1} \mathrm{H}$ NMR $\left(500 \mathrm{MHz}\right.$, Acetone- $\left.d_{6}\right)$
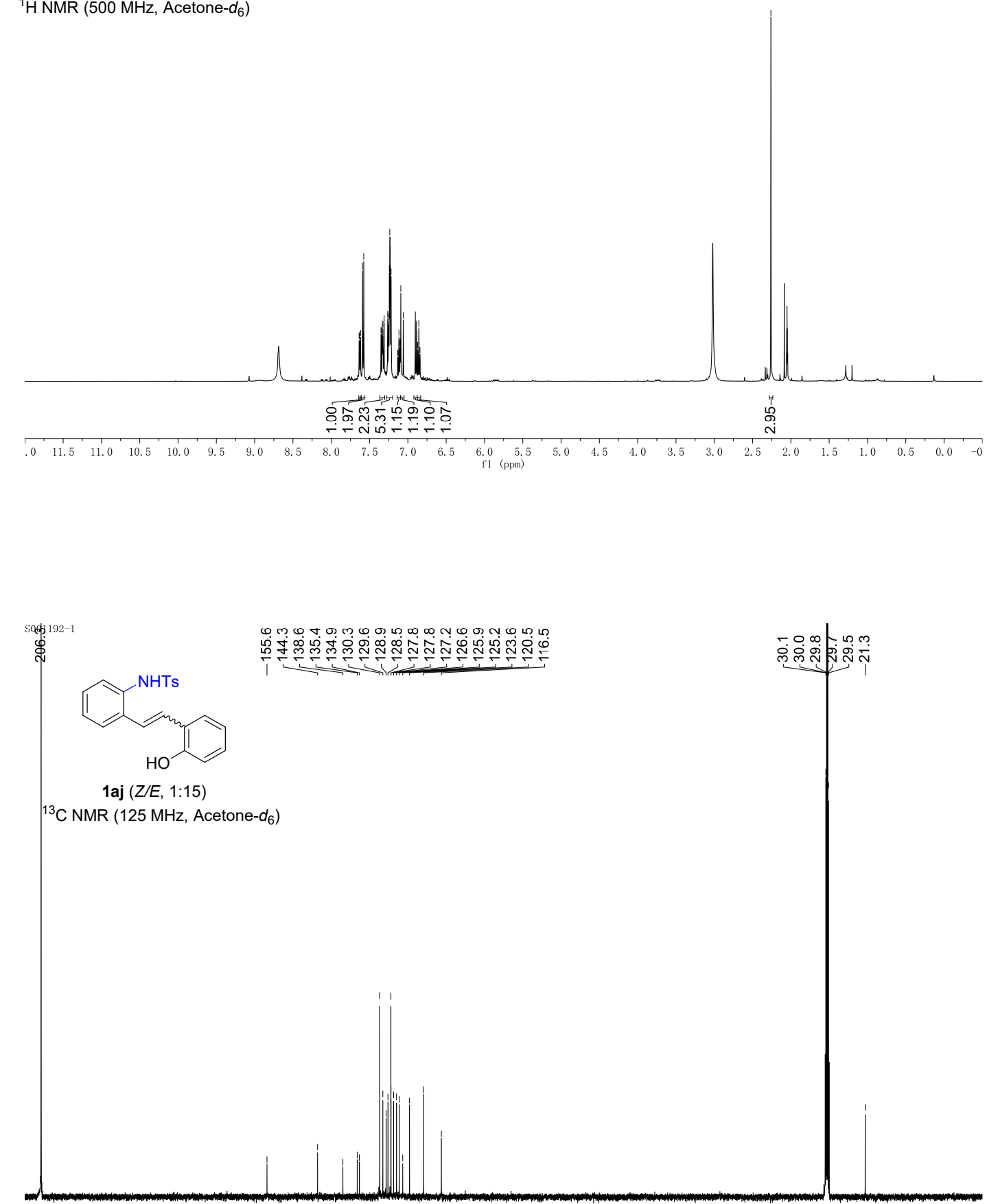

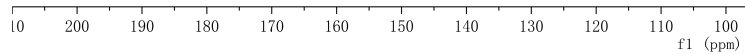




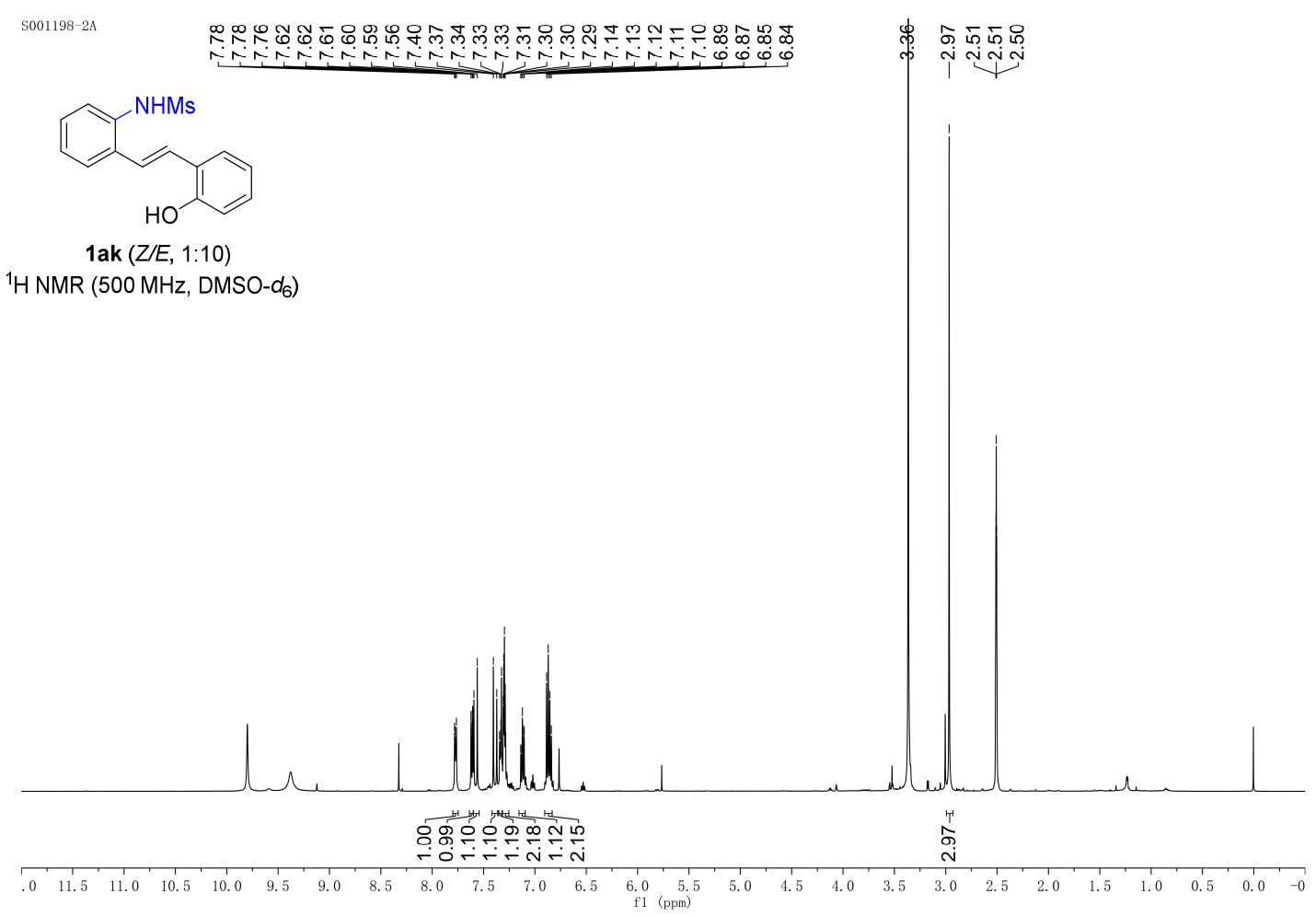

S001198-2A

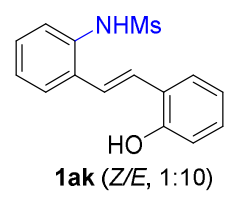

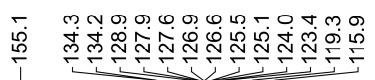

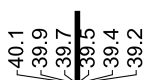

${ }^{3} \mathrm{C}$ NMR (125 MHz, DMSO-d $)$

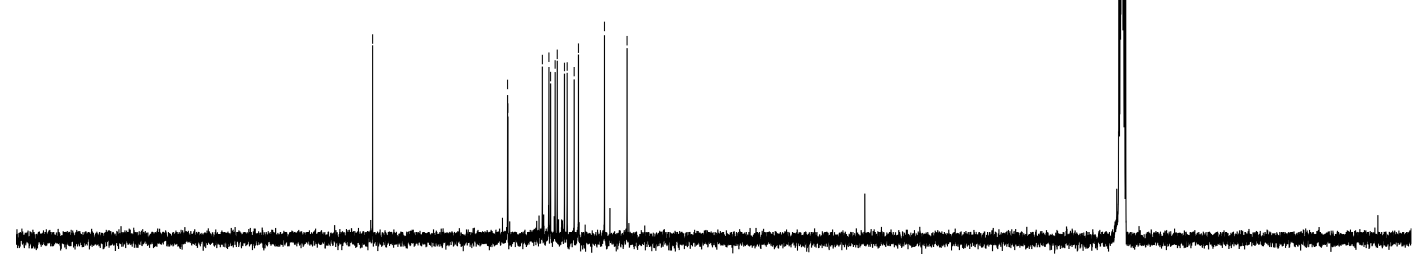

\begin{tabular}{llllllllllll}
\hline 10 & 200 & 190 & 180 & 170 & 160 & 150 & 140 & 130 & 120 & 110 & 100 \\
$\mathrm{f} 1$ & $1 \mathrm{ppm})$
\end{tabular} 
zj2009151-2
zj2009151-2

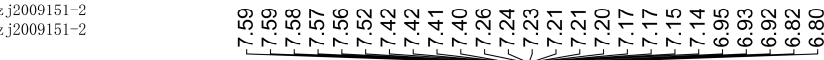

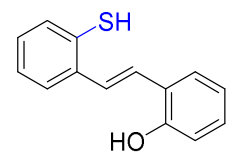

1al

${ }^{1} \mathrm{H} \mathrm{NMR}\left(500 \mathrm{MHz}, \mathrm{CDCl}_{3}\right)$

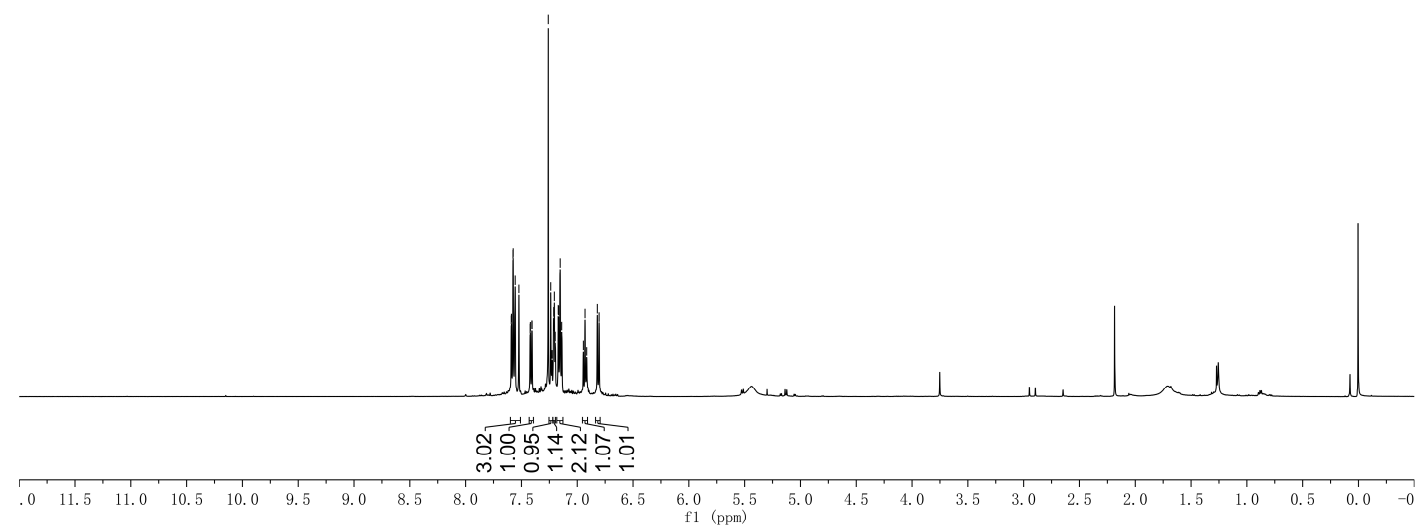

$\mathrm{zj} 2009151-2$
$\mathrm{zj} 2009151-2$

m户̃丶

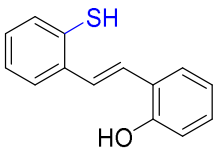

1al

${ }^{13} \mathrm{C} \mathrm{NMR}\left(125 \mathrm{MHz}, \mathrm{CDCl}_{3}\right)$

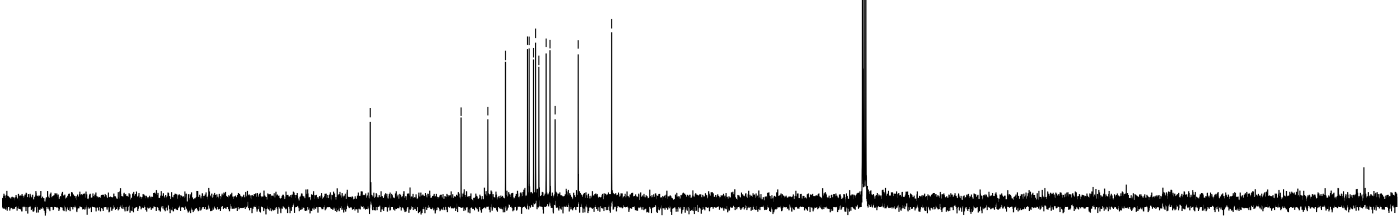

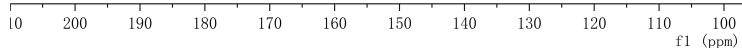


ZJ200841-1

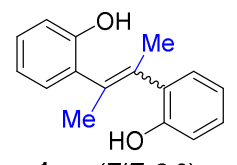

1 am (ZIE, 2:3)

${ }^{1} \mathrm{H}$ NMR $(500 \mathrm{MHz}$, Methanol-d $)$

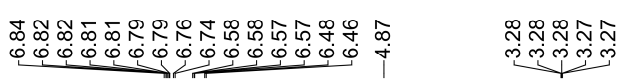
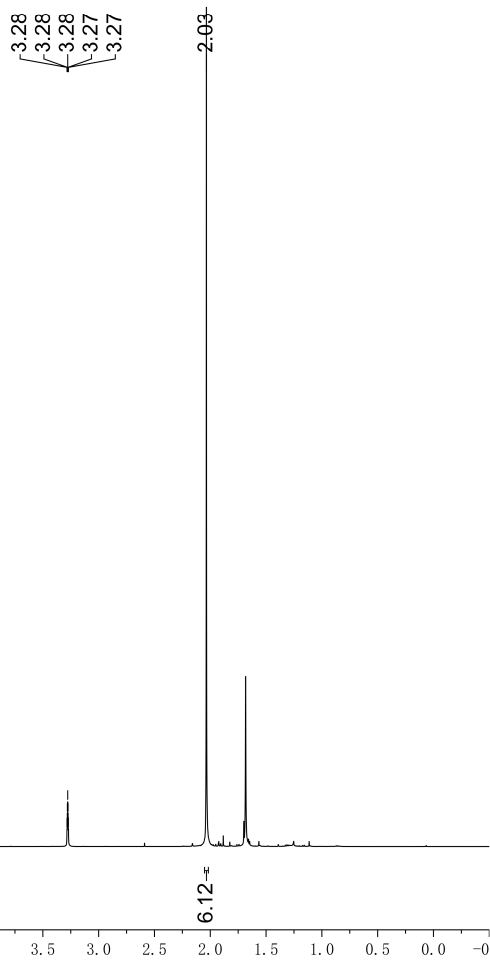

Nol

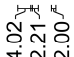

\begin{tabular}{llllllllllllllll}
\hline 0 & 11.5 & 11.0 & 10.5 & 10.0 & 9.5 & 9.0 & 8.5 & 8.0 & 7.5 & 7.0 & 6.5 & 6.0 & 5.5 \\
f1 & $(\mathrm{ppm})$
\end{tabular}

ZJ200841-1

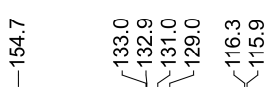

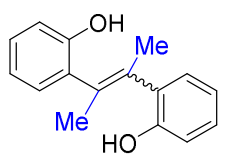

1am (ZIE, 2:3)

${ }^{13} \mathrm{C}$ NMR $\left(125 \mathrm{MHz}\right.$, Methanol- $\left.d_{4}\right)$

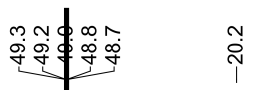




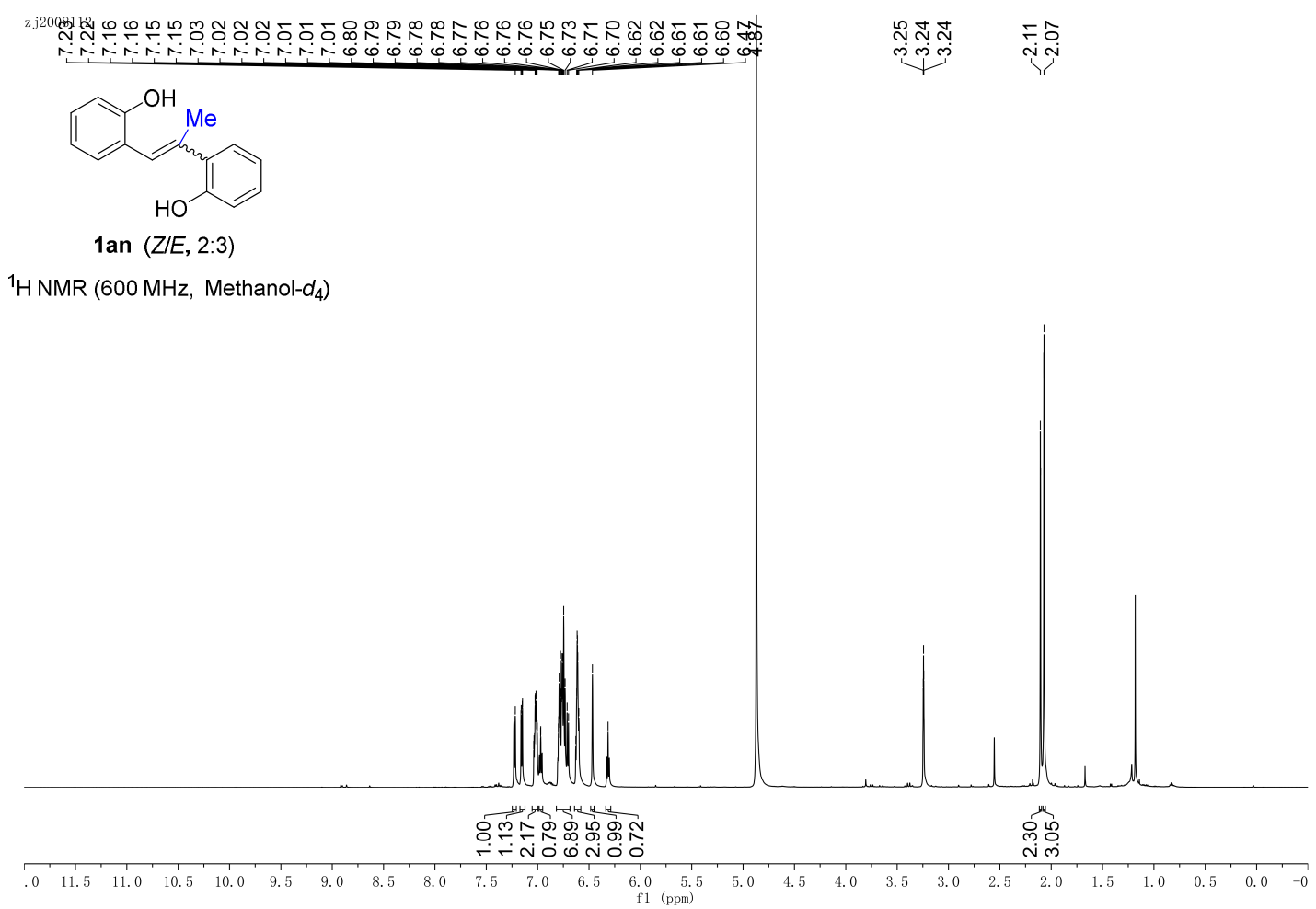

zj2008112
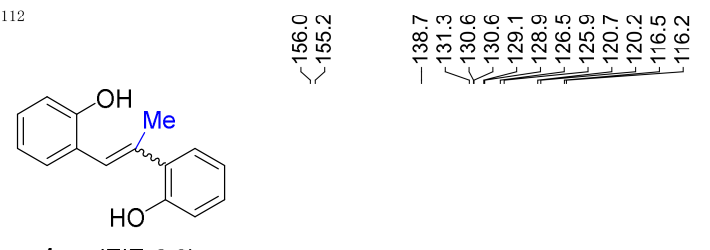

1an (ZIE, 2:3)

${ }^{13} \mathrm{C}$ NMR $\left(125 \mathrm{MHz}\right.$, Methanol- $\left.d_{4}\right)$

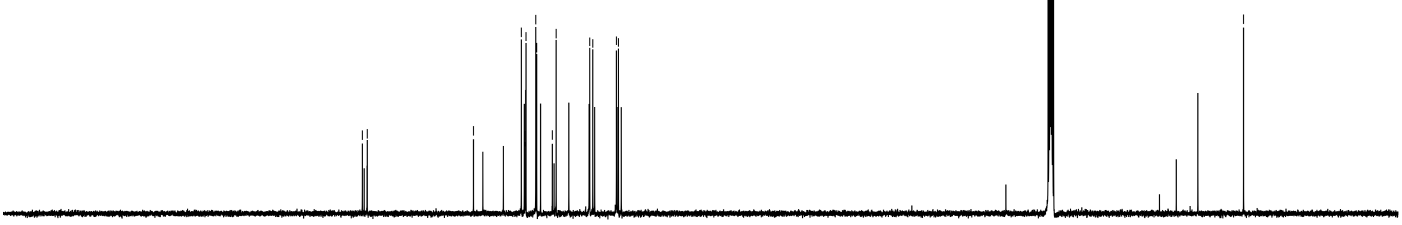

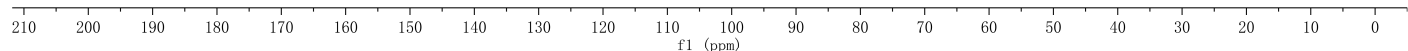




\section{NMR spectra of 2a-2an}

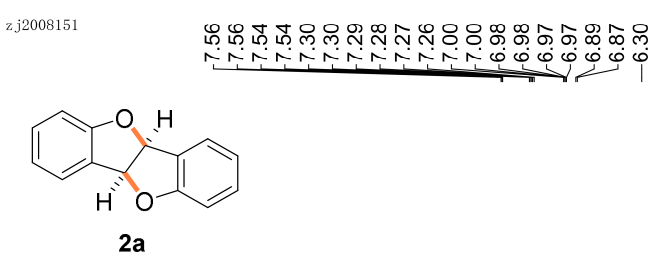

${ }^{1} \mathrm{H}$ NMR $\left(600 \mathrm{MHz}, \mathrm{CDCl}_{3}\right)$

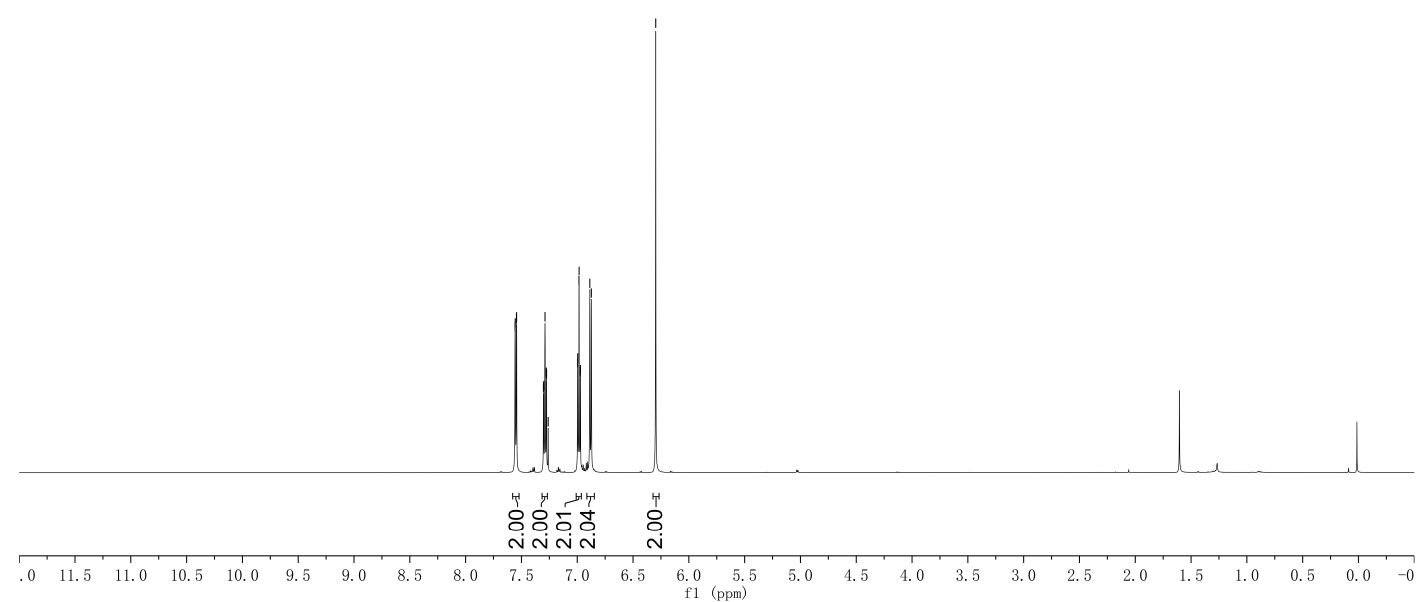

zj2008151

\begin{tabular}{|c|c|c|c|}
\hline$\check{8}$ & 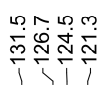 & $\frac{\circ}{\stackrel{\Gamma}{\digamma}}$ & $\begin{array}{l}0 \\
\dot{0} N \\
1\end{array}$ \\
\hline
\end{tabular}<smiles>c1ccc2c(c1)O[C@@H]1c3ccccc3O[C@@H]21</smiles>

2a

${ }^{13} \mathrm{C} \mathrm{NMR}\left(150 \mathrm{MHz}, \mathrm{CDCl}_{3}\right)$

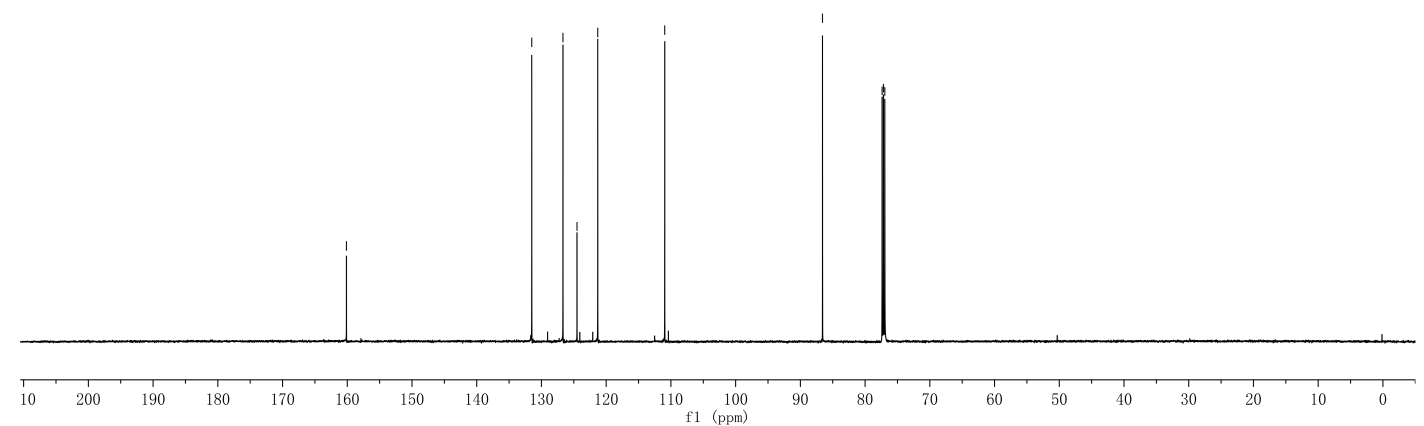




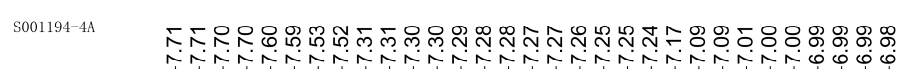

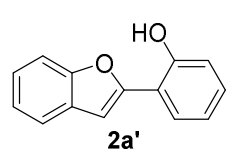

${ }^{1} \mathrm{H} \mathrm{NMR}\left(600 \mathrm{MHz}, \mathrm{CDCl}_{3}\right)$

S001194-4A

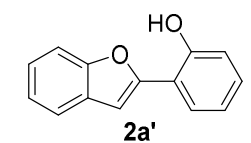

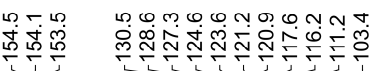

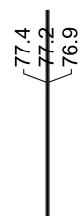

${ }^{13} \mathrm{C} \mathrm{NMR}\left(150 \mathrm{MHz}, \mathrm{CDCl}_{3}\right)$

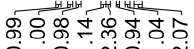

\begin{tabular}{rrrrrrrrrrrrrrrrrrrrrrrrrrrr}
\hline .0 & 11.5 & 11.0 & 10.5 & 10.0 & 9.5 & 9.0 & 8.5 & 8.0 & 7.5 & 7.0 & 6.5 & 6.0 & 5.5 & 5.0 & 4.5 & 4.0 & 3.5 & 3.0 & 2.5 & 2.0 & 1.5 & 1.0 & 0.5 & 0.0 & -0
\end{tabular}

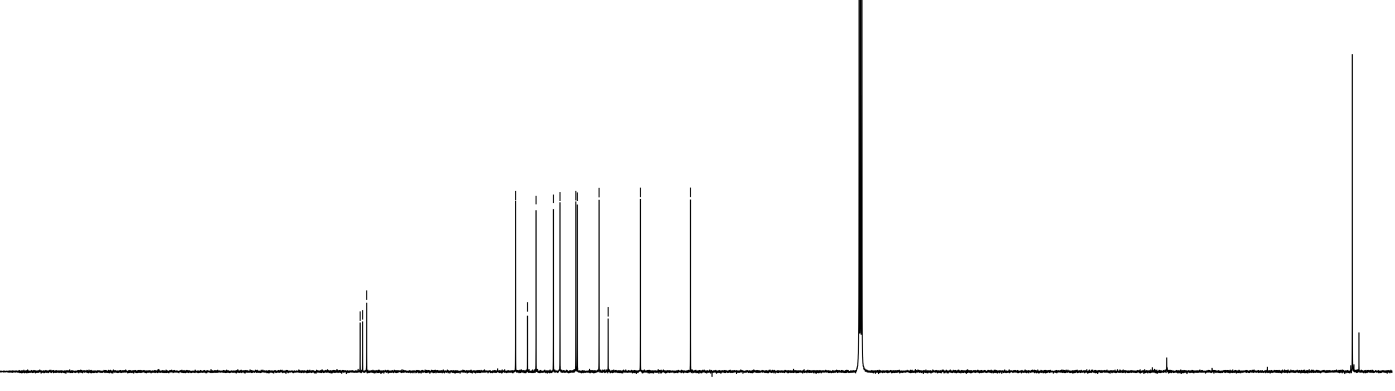

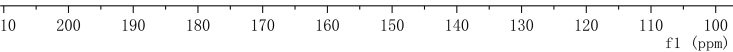


2J2007112

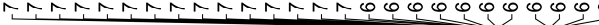

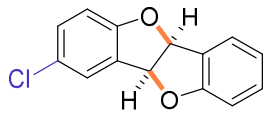

2b

${ }^{1} \mathrm{H} \mathrm{NMR}\left(500 \mathrm{MHz}, \mathrm{CDCl}_{3}\right)$

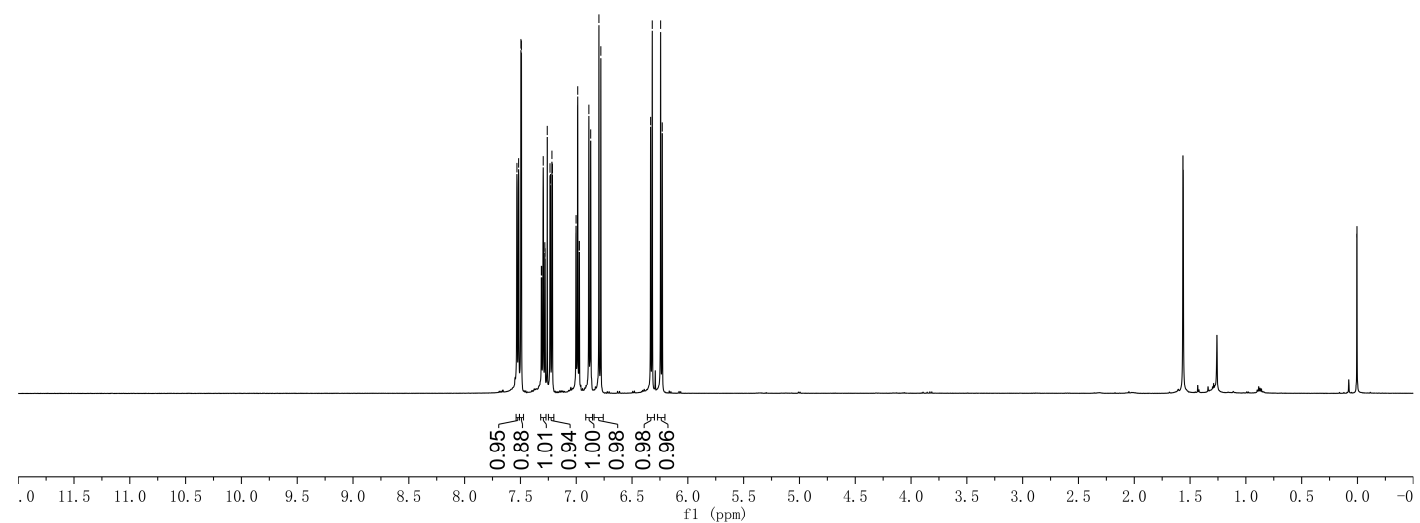

ZJ2007112

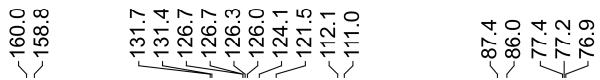

(l)

2b

${ }^{13} \mathrm{C} \mathrm{NMR}\left(125 \mathrm{MHz}, \mathrm{CDCl}_{3}\right)$

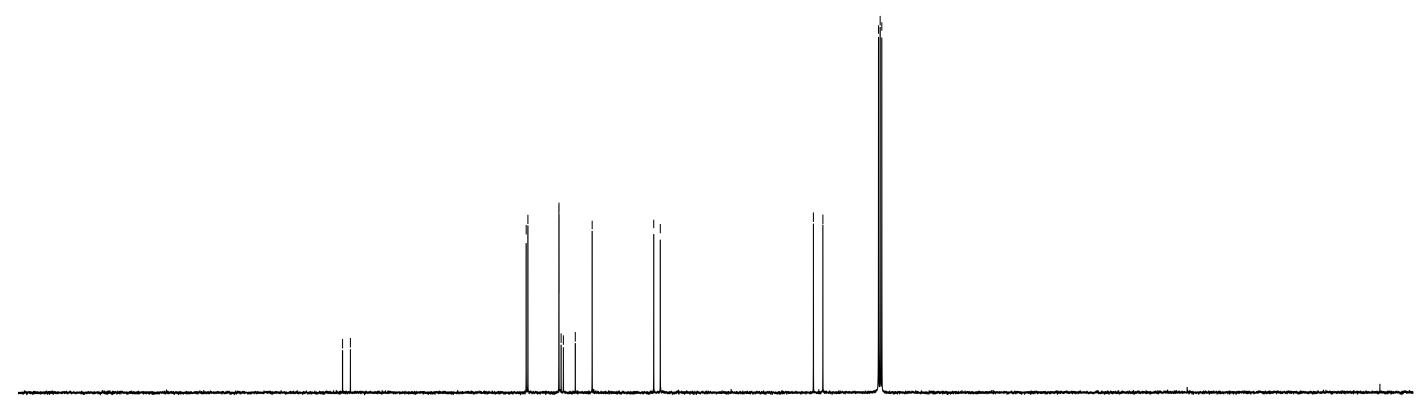

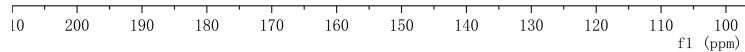




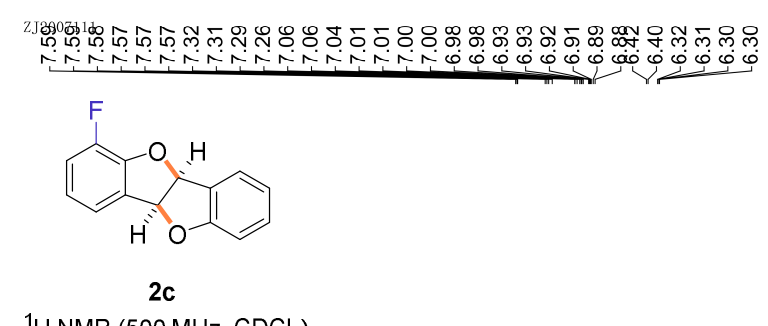

${ }^{1} \mathrm{H} \mathrm{NMR}\left(500 \mathrm{MHz}, \mathrm{CDCl}_{3}\right)$

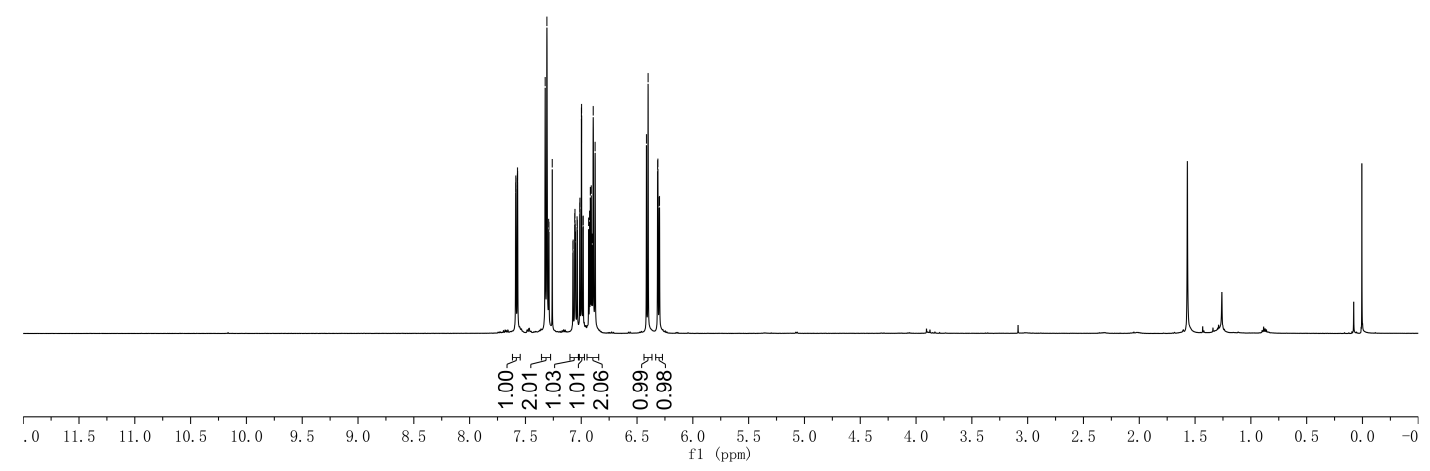

ZJ2007111

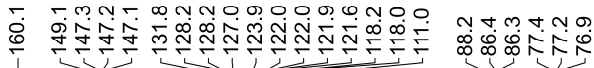

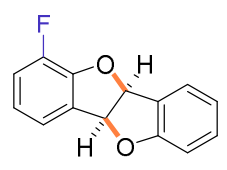

2c

${ }^{13} \mathrm{C}$ NMR $\left(125 \mathrm{MHz}, \mathrm{CDCl}_{3}\right)$

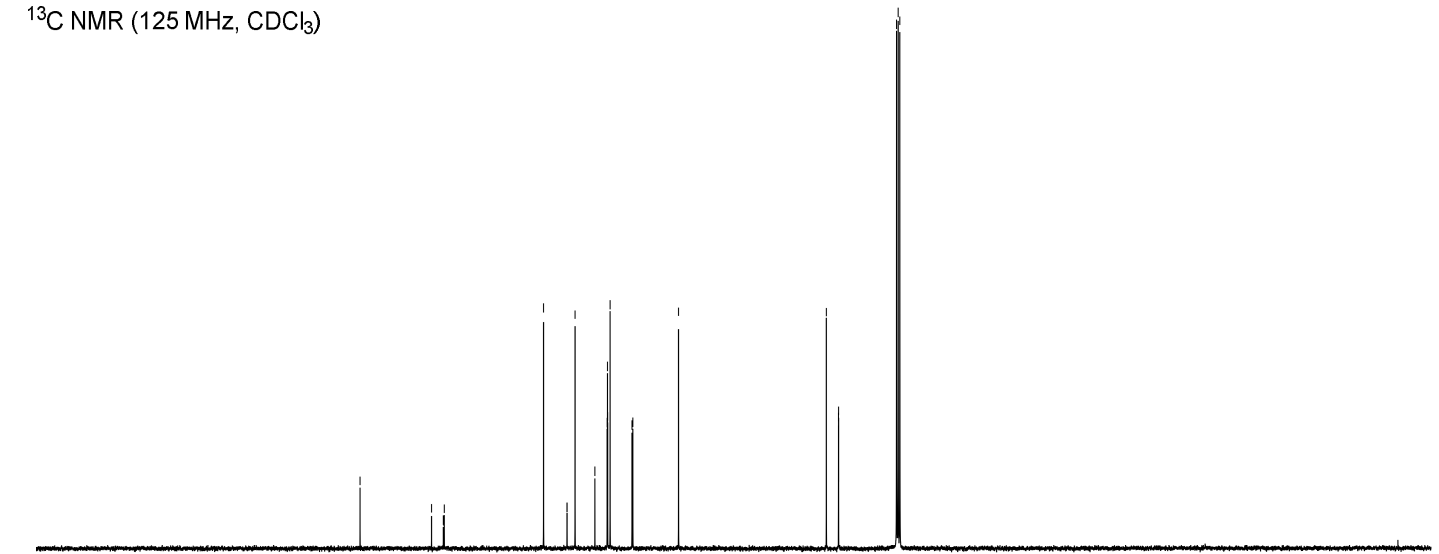

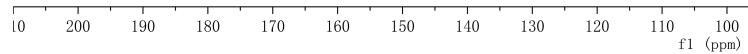


zj2007111F<smiles>Fc1cccc2c1O[C@H]1c3ccccc3O[C@H]21</smiles>

2c

${ }^{19} \mathrm{~F} \mathrm{NMR}\left(471 \mathrm{MHz}, \mathrm{CDCl}_{3}\right)$

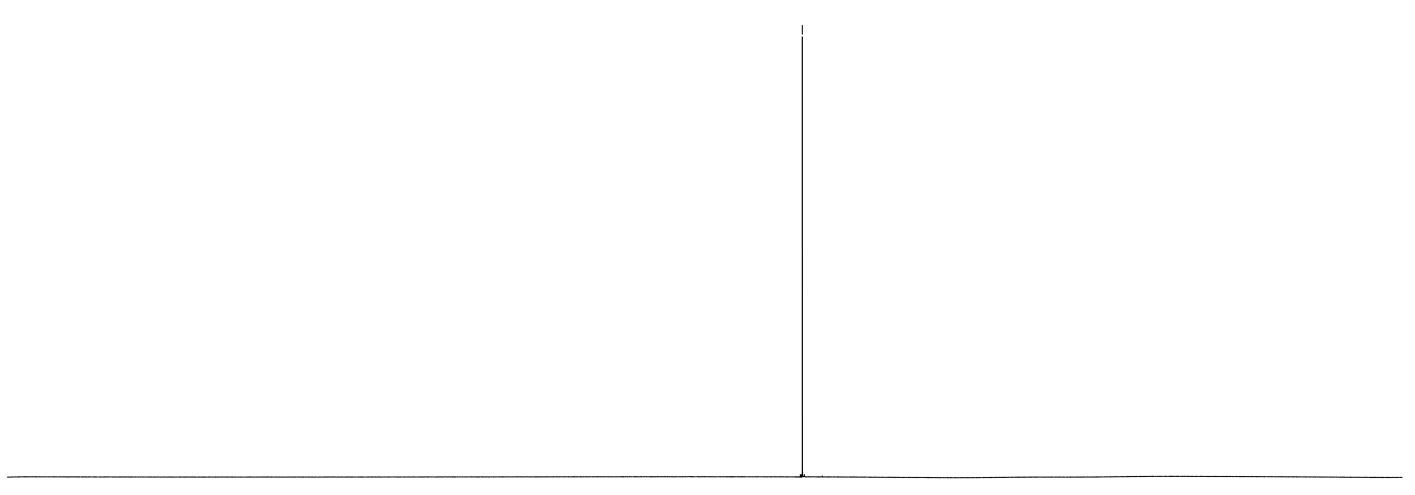

\begin{tabular}{llllllllllllllllllllllllllllll}
\hline & 0 & -10 & -20 & -30 & -40 & -50 & -60 & -70 & -80 & -90 & -100 & -110 & -120 & -130 & -140 & -150 & -160 & -170 & -180 & -190 & -200 & -210 & -220 & -230 & -2 \\
\hline
\end{tabular} 
ZJ2007113

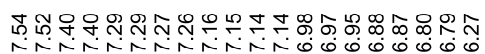

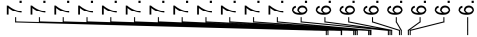

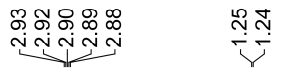

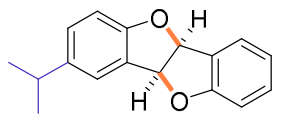

2d

${ }^{1} \mathrm{H}$ NMR $\left(500 \mathrm{MHz}, \mathrm{CDCl}_{3}\right)$

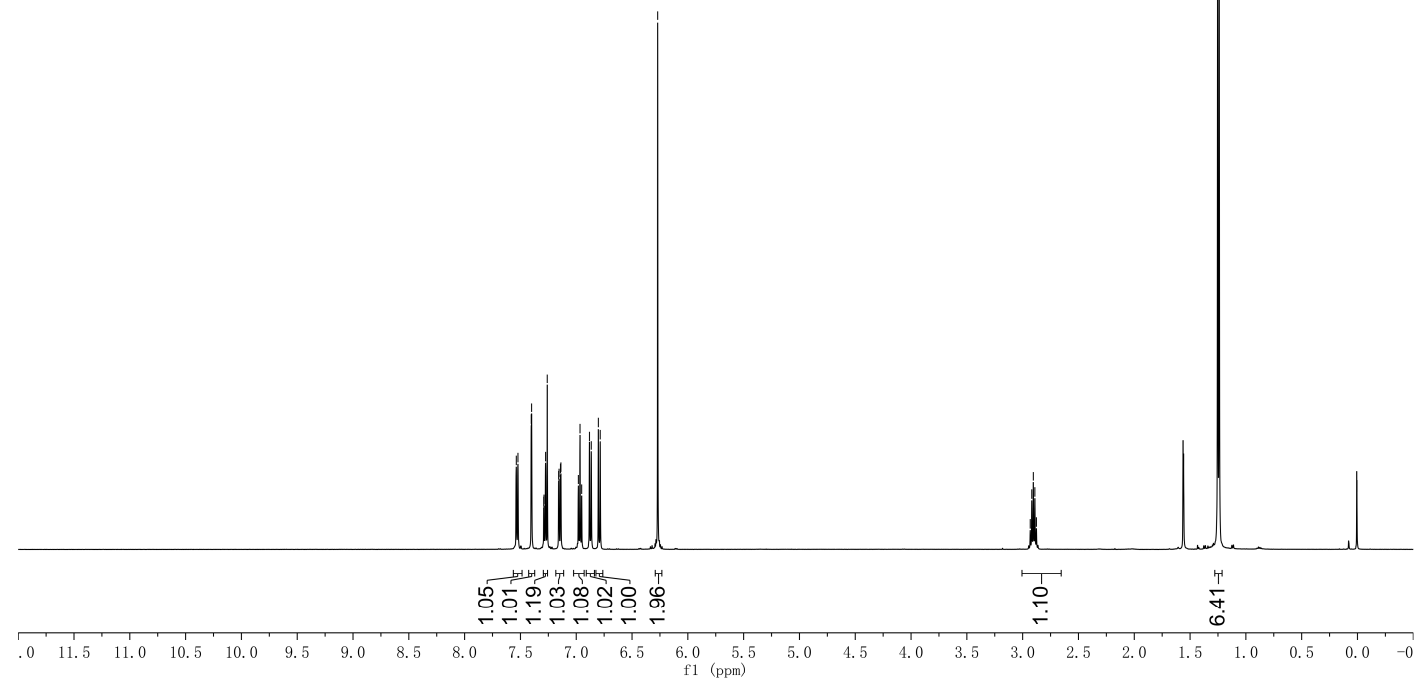

ZJ2007113

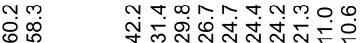

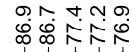

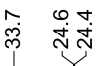

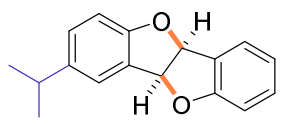

2d

${ }^{13} \mathrm{C}$ NMR $\left(125 \mathrm{MHz}, \mathrm{CDCl}_{3}\right)$

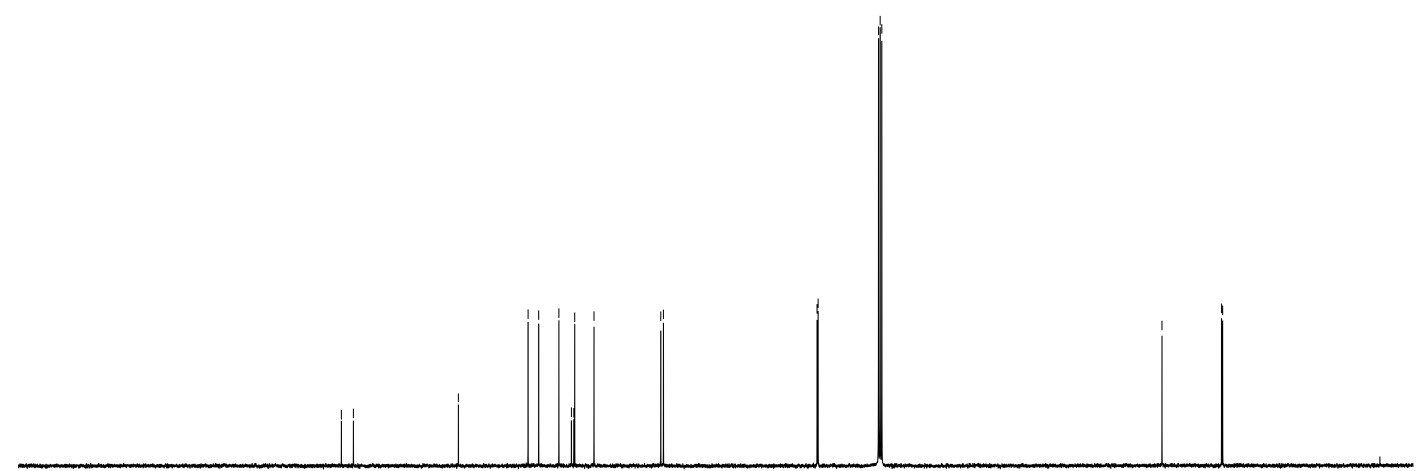

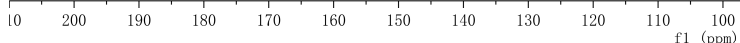




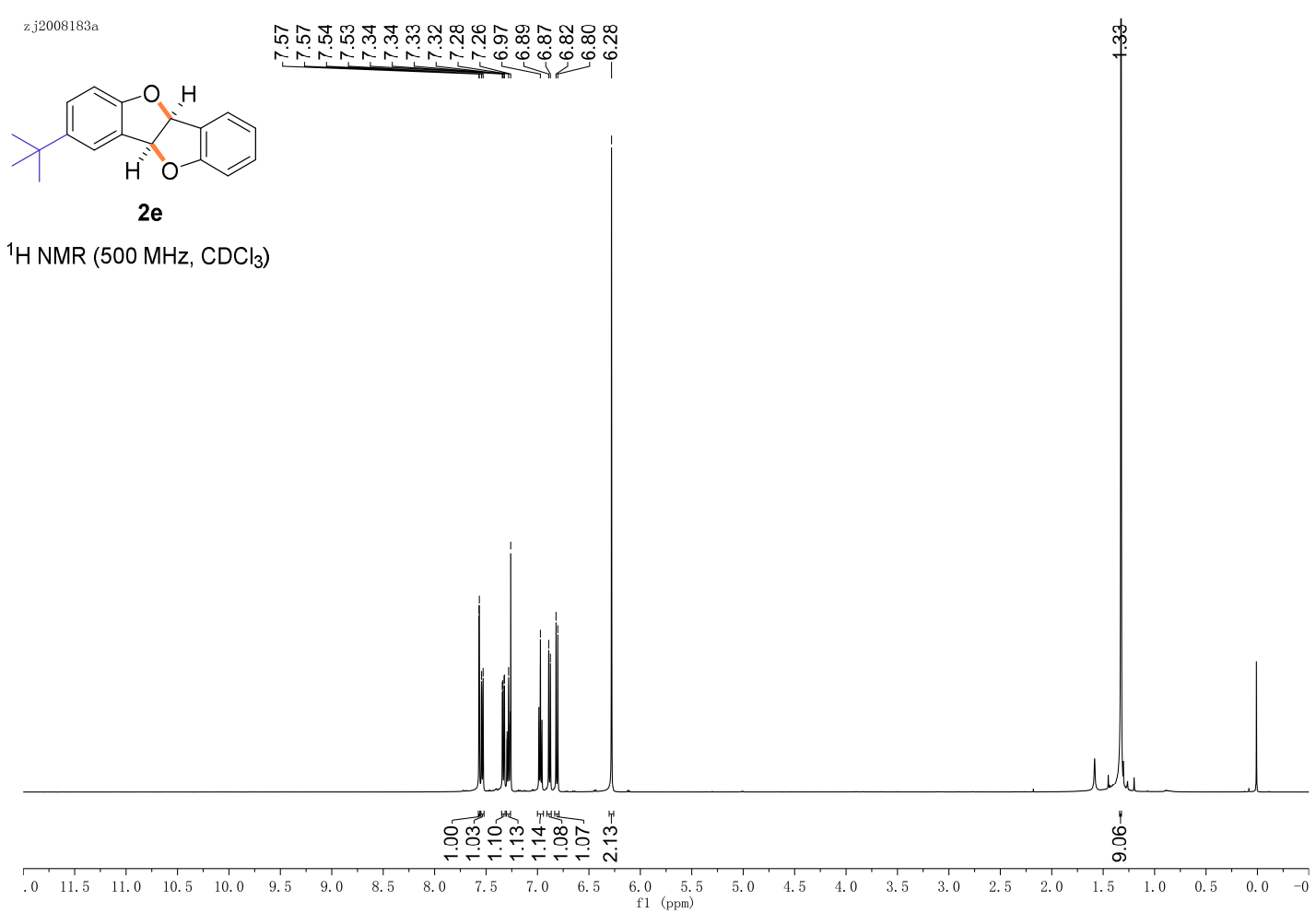

zj2008183a
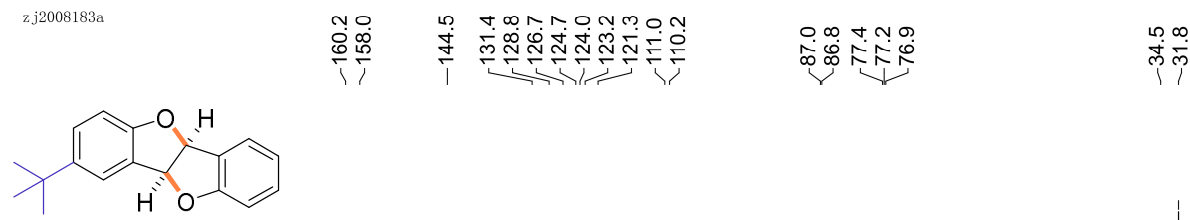

$2 \mathrm{e}$

${ }^{13} \mathrm{C}$ NMR $\left(125 \mathrm{MHz}, \mathrm{CDCl}_{3}\right)$

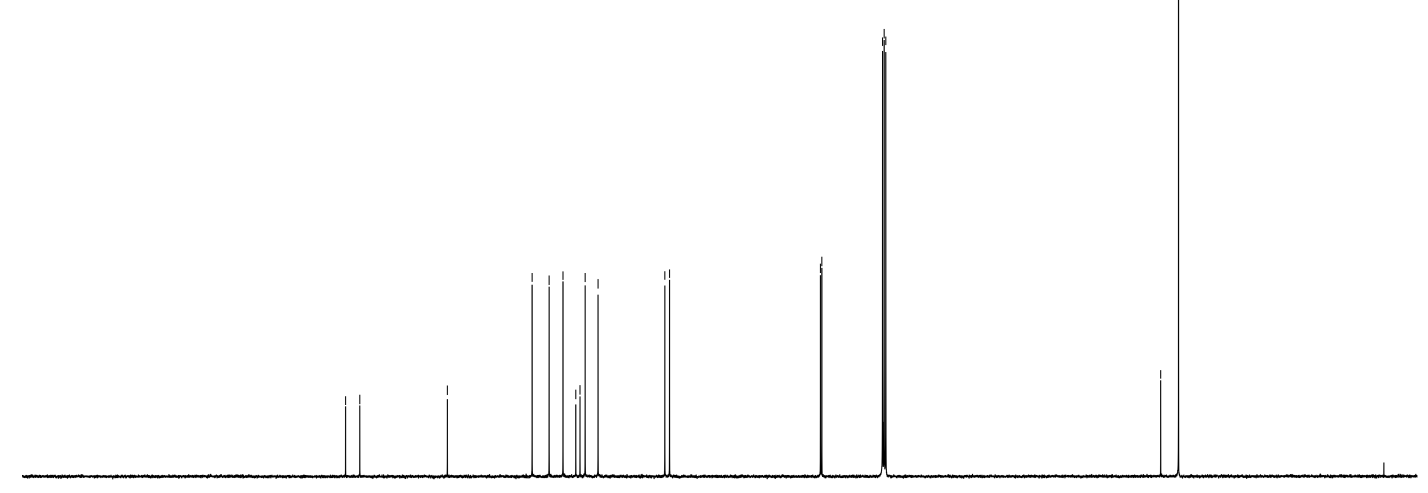

\begin{tabular}{llllllllllll}
\hline 10 & 200 & 190 & 180 & 170 & 160 & 150 & 140 & 130 & 120 & 110 & 100 \\
$\mathrm{f} 1$ & $\begin{array}{l}1 \\
(\mathrm{ppm})\end{array}$
\end{tabular} 


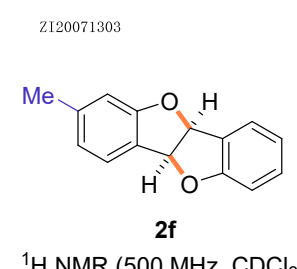

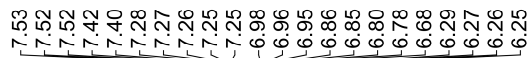

${ }^{1} \mathrm{H}$ NMR $\left(500 \mathrm{MHz}, \mathrm{CDCl}_{3}\right)$

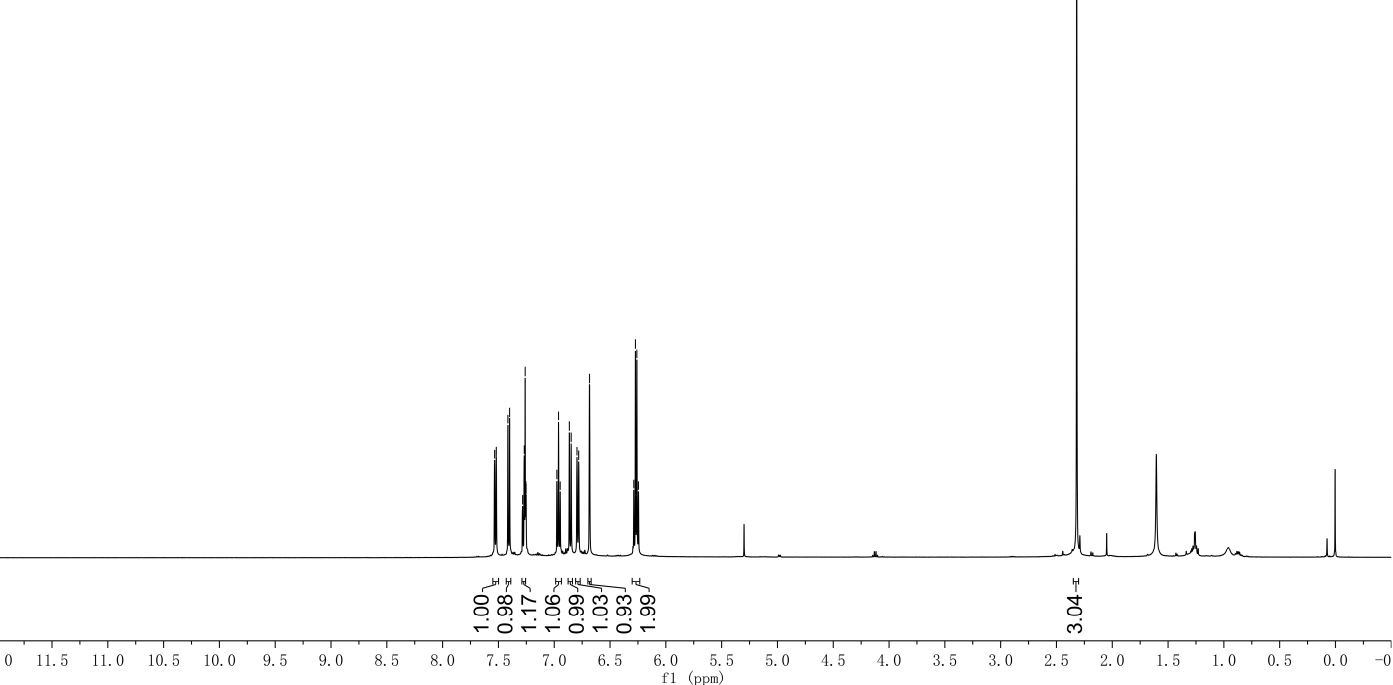

2I20071303

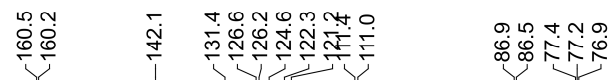

$\stackrel{\leftrightarrow}{i}$

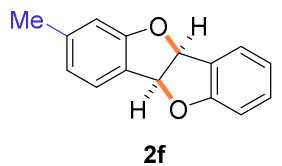

${ }^{13} \mathrm{C} \mathrm{NMR}\left(125 \mathrm{MHz}, \mathrm{CDCl}_{3}\right)$

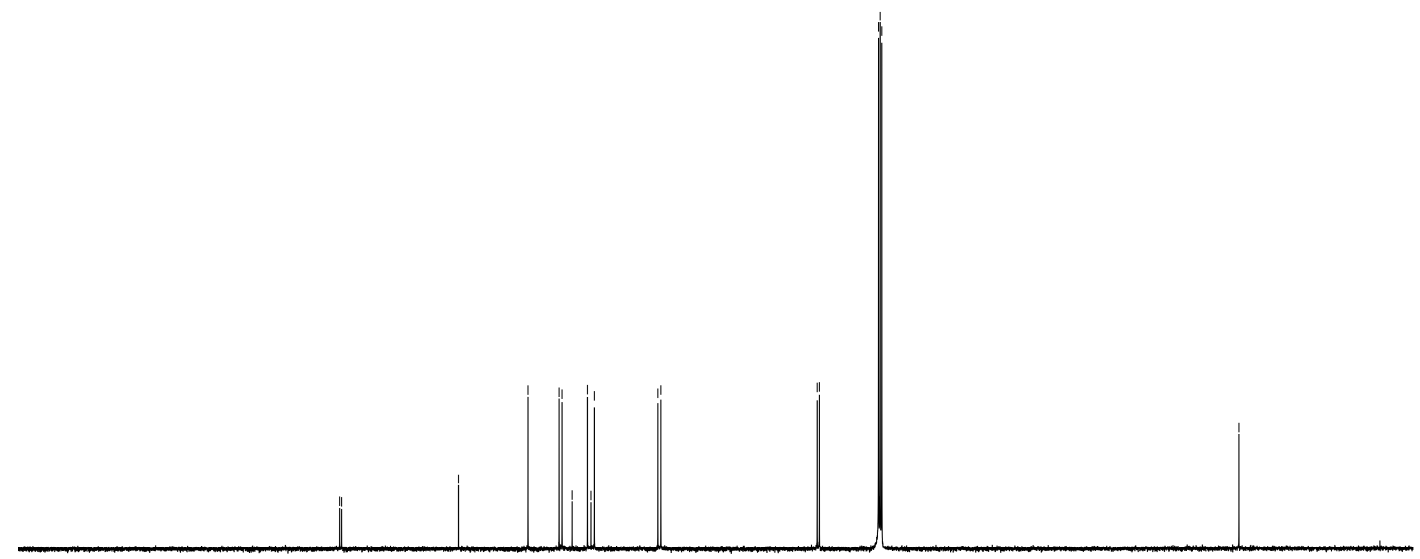

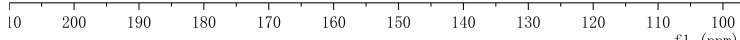




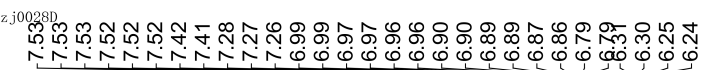

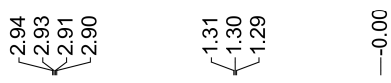

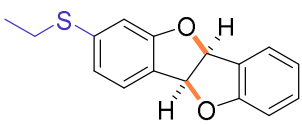

$2 \mathrm{~g}$

${ }^{1} \mathrm{H} \mathrm{NMR}\left(600 \mathrm{MHz}, \mathrm{CDCl}_{3}\right)$

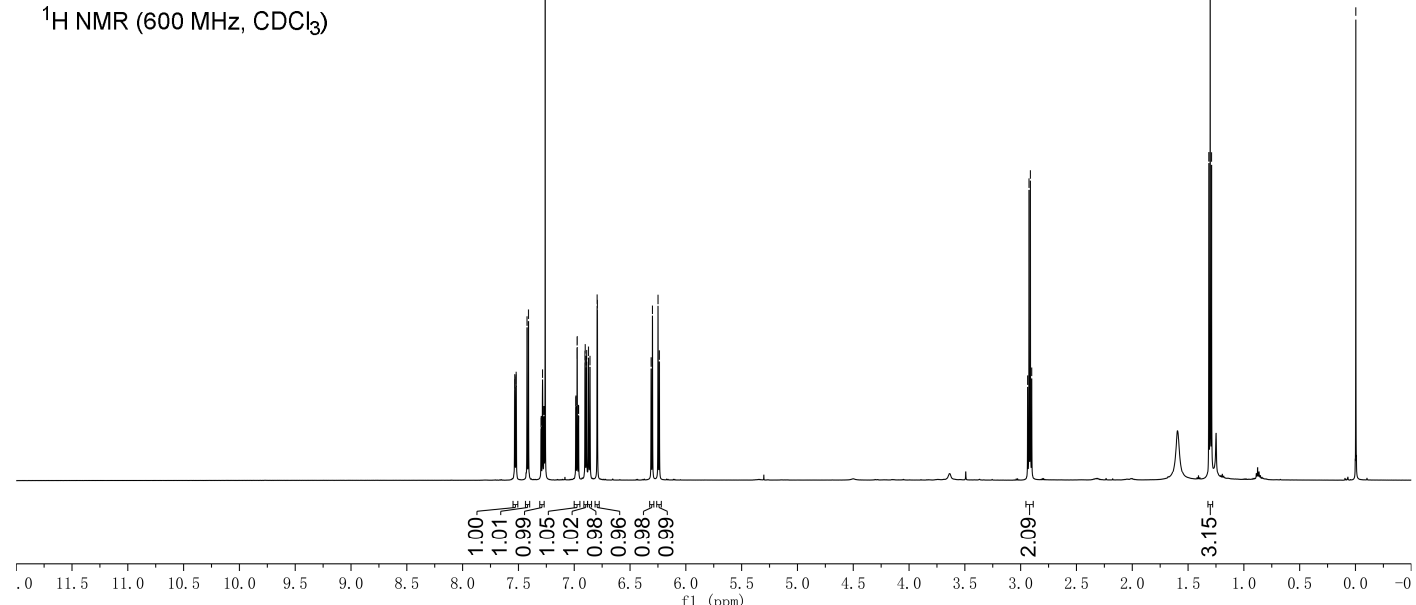

zj0028

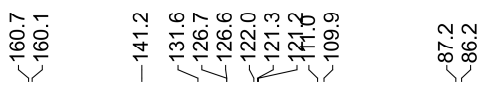

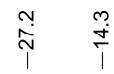

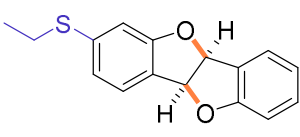

2g

${ }^{13} \mathrm{C} \mathrm{NMR}\left(150 \mathrm{MHz}, \mathrm{CDCl}_{3}\right)$

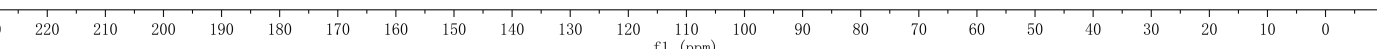




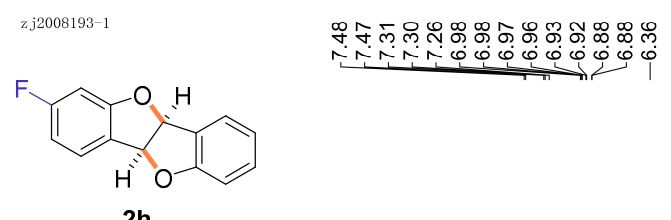

${ }^{1} \mathrm{H}$ NMR $\left(500 \mathrm{MHz}, \mathrm{CDCl}_{3}\right)$
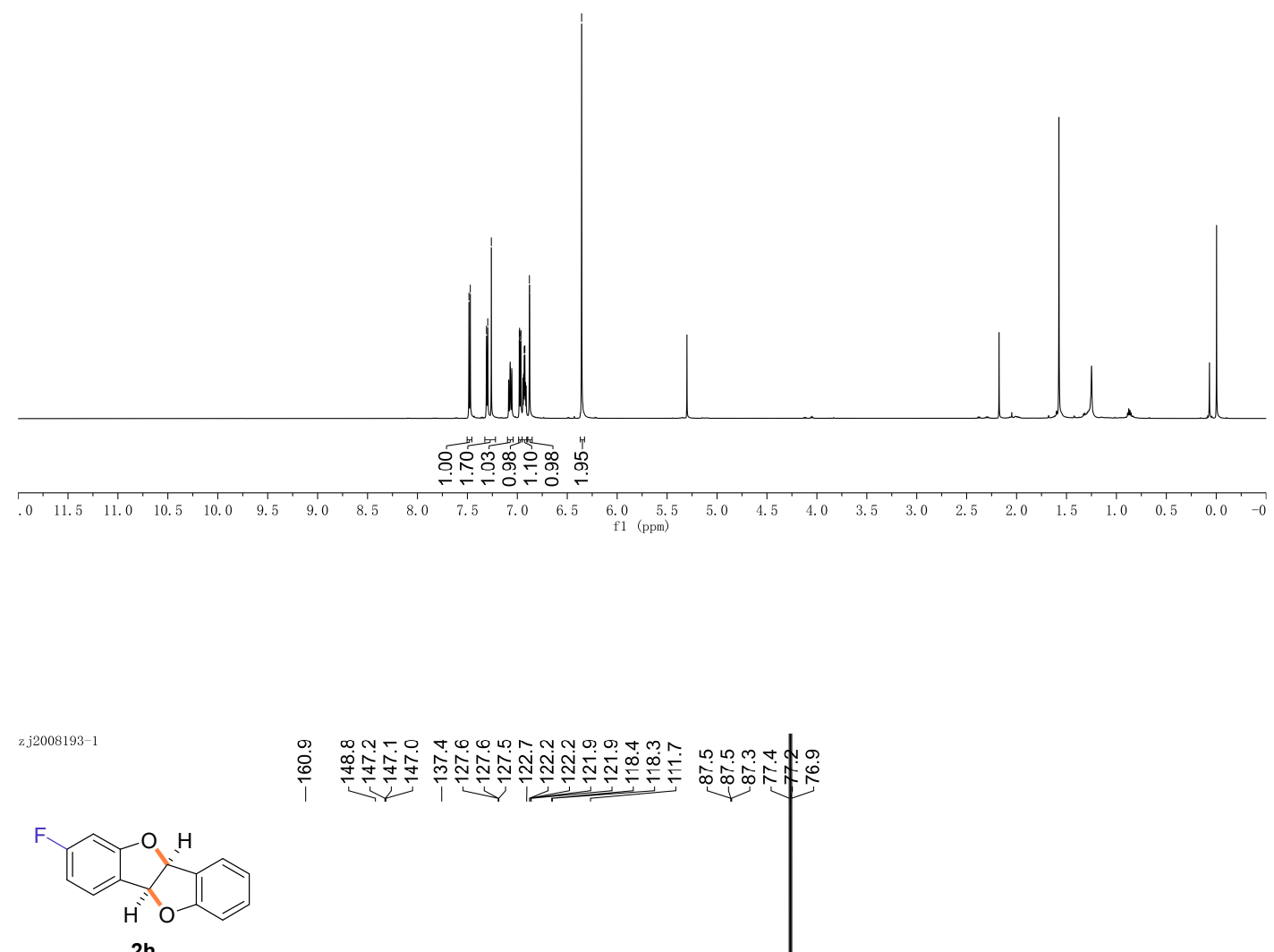

至

${ }^{13} \mathrm{C}$ NMR $\left(125 \mathrm{MHz}, \mathrm{CDCl}_{3}\right)$

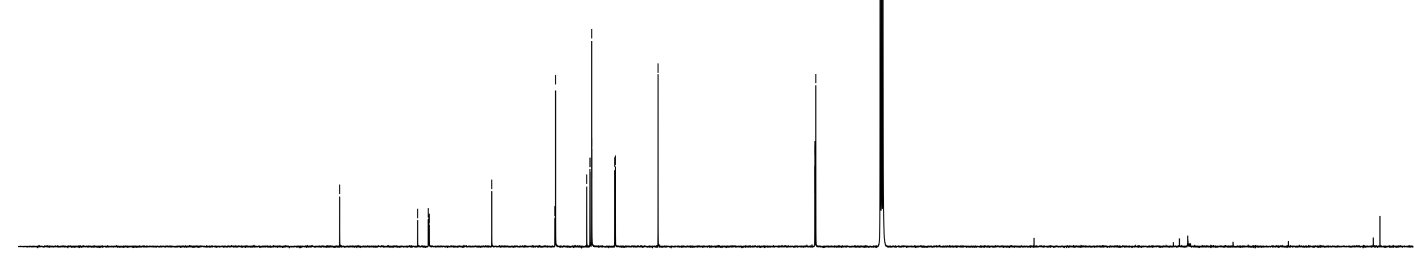

\begin{tabular}{llllllllllll}
\hline 10 & 200 & 190 & 180 & 170 & 160 & 150 & 140 & 130 & 120 & 110 & $\begin{array}{r}1 \\
100\end{array}$ \\
$(\mathrm{pmm})$
\end{tabular} 
z j2008193-1

กั่

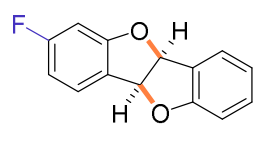

2h

${ }^{19} \mathrm{~F} \mathrm{NMR}\left(471 \mathrm{MHz}, \mathrm{CDCl}_{3}\right)$

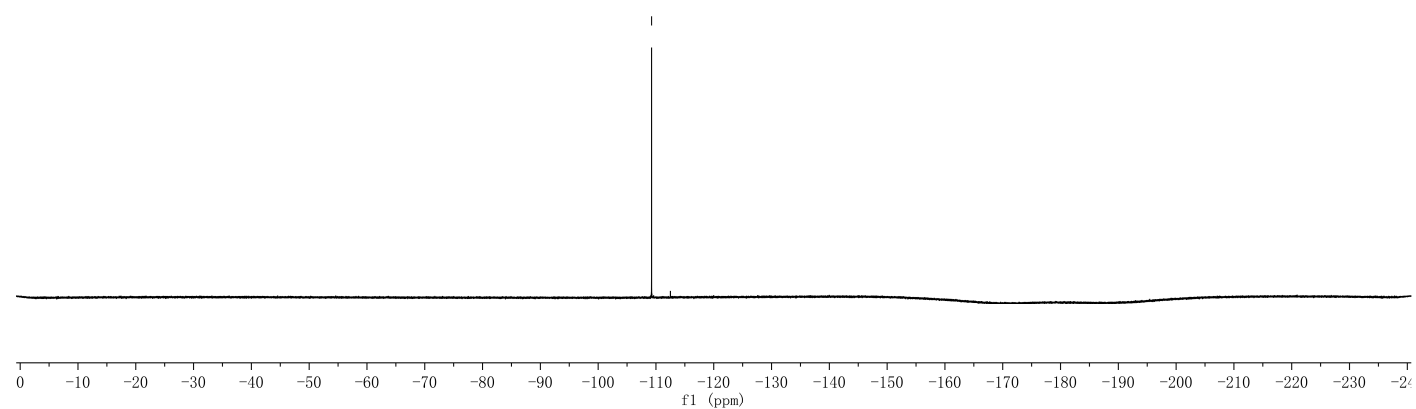




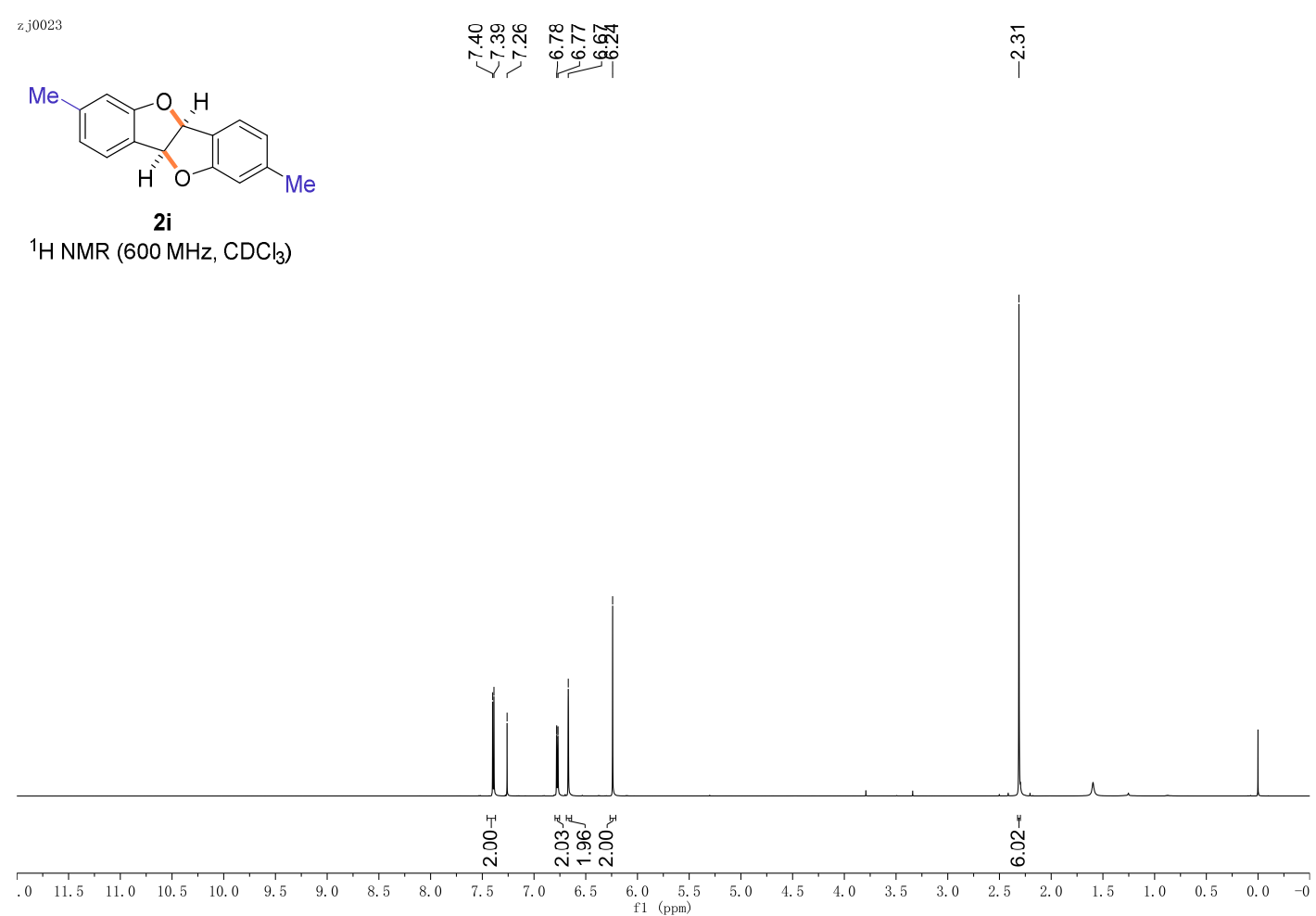

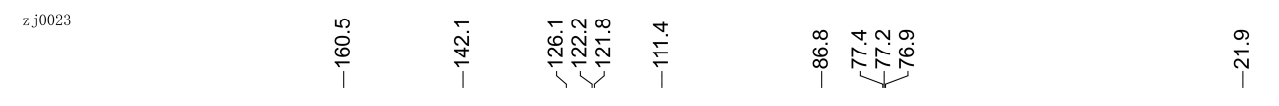

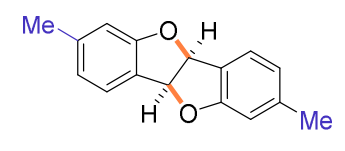

${ }^{13} \mathrm{C}$ NMR $\left(150 \mathrm{MHz}, \mathrm{CDCl}_{3}\right)$

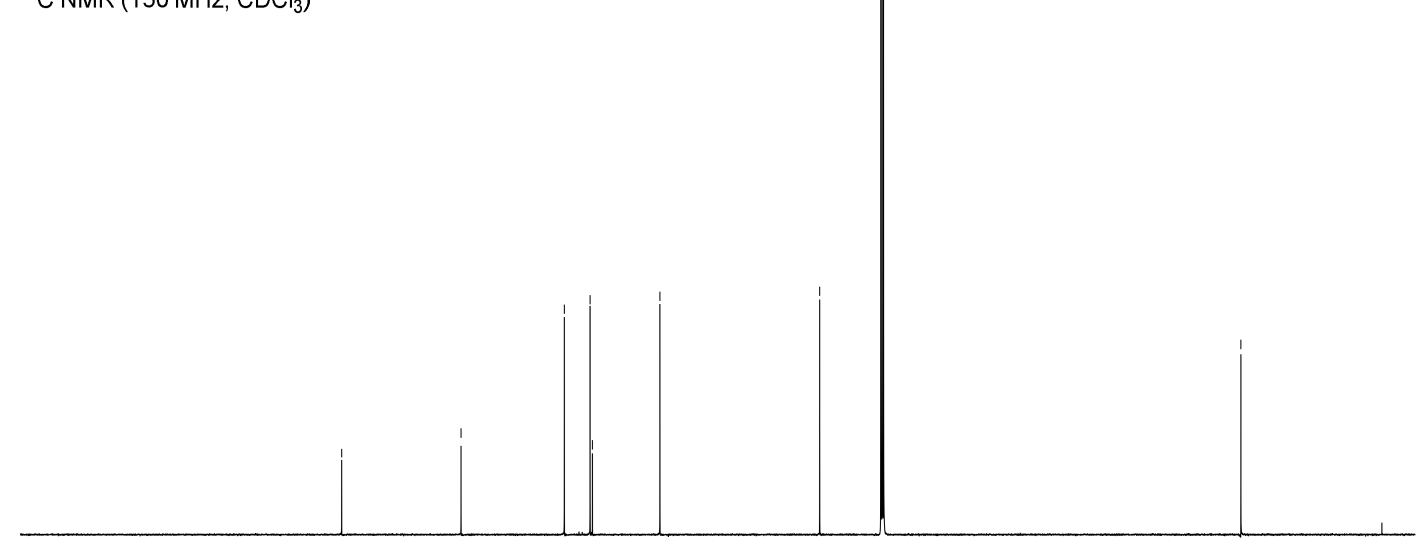

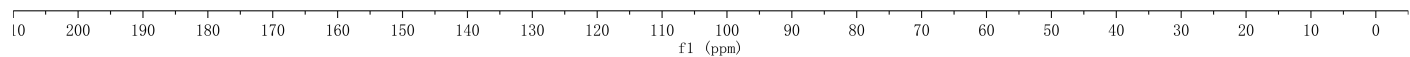




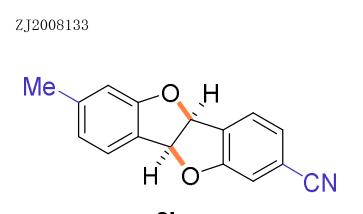

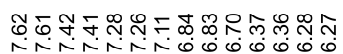

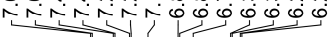

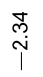

${ }^{1} \mathrm{H} \mathrm{NMR}\left(500 \mathrm{MHz}, \mathrm{CDCl}_{3}\right)$

(H)

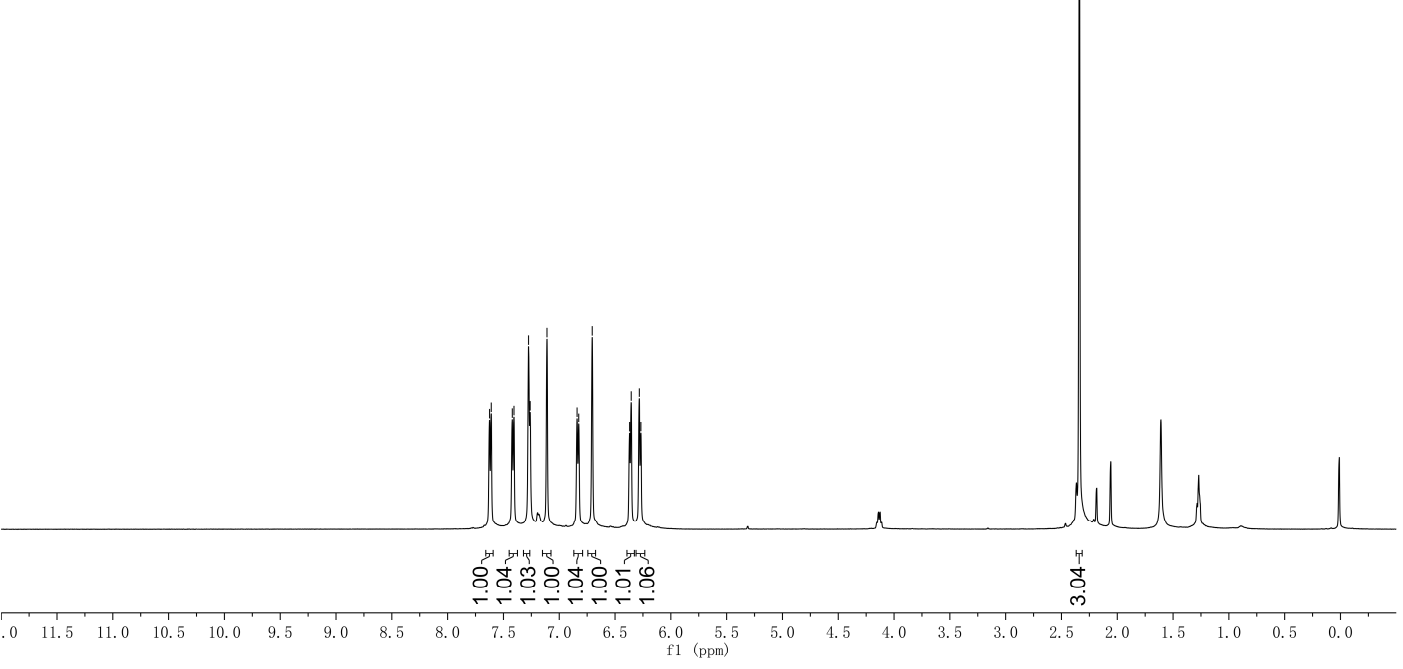

2J2008133

至

$\stackrel{\leftrightarrow}{i}$

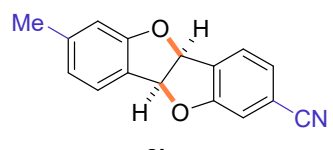

2j

${ }^{13} \mathrm{C} \mathrm{NMR}\left(125 \mathrm{MHz}, \mathrm{CDCl}_{3}\right)$

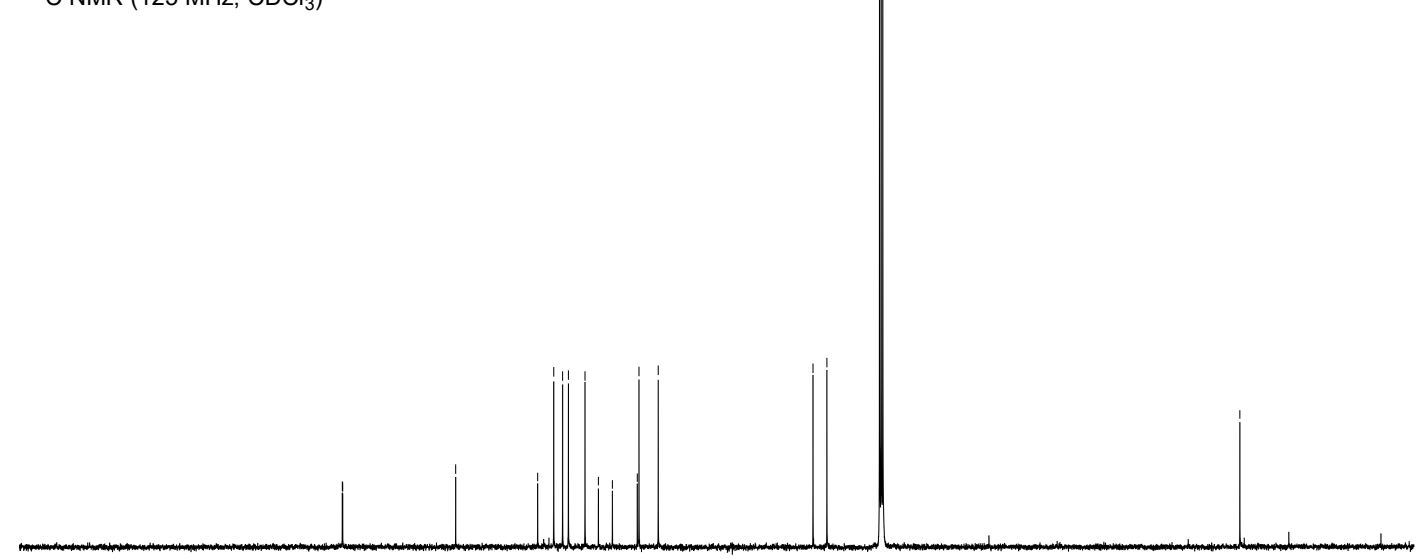

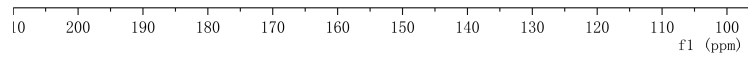


ZJ200853

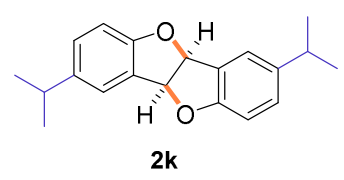

${ }^{1} \mathrm{H}$ NMR $\left(500 \mathrm{MHz}, \mathrm{CDCl}_{3}\right)$

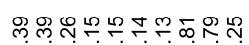

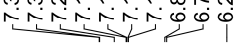

ஜㄷำ

نู่

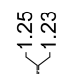

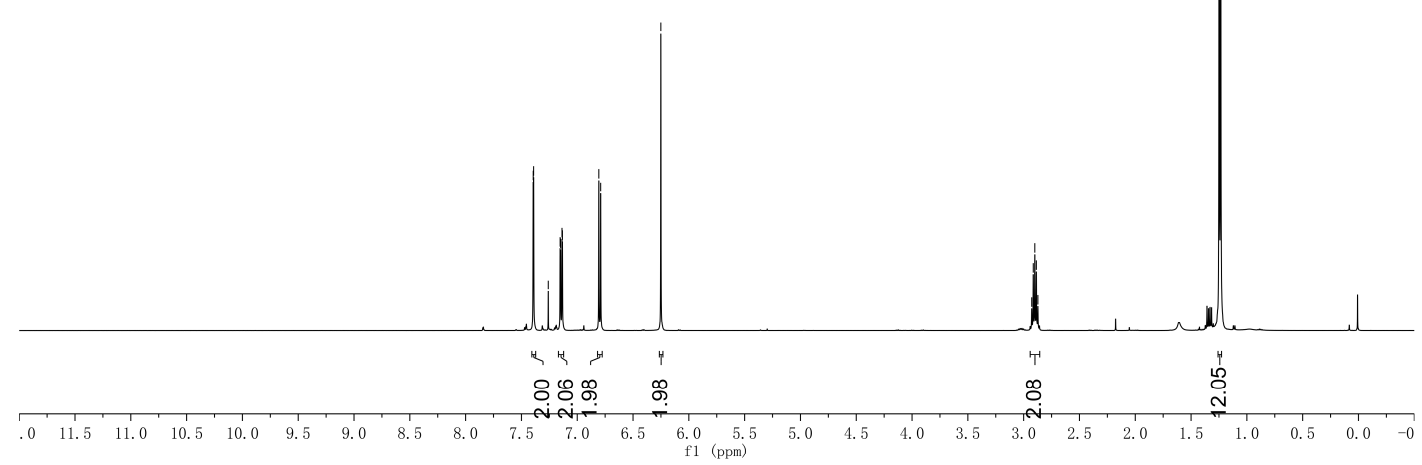

2J200853
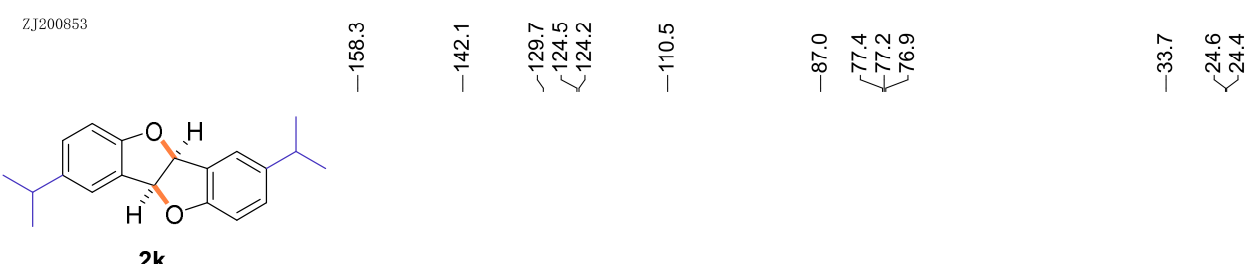

${ }^{13} \mathrm{C} \mathrm{NMR}\left(125 \mathrm{MHz}, \mathrm{CDCl}_{3}\right)$

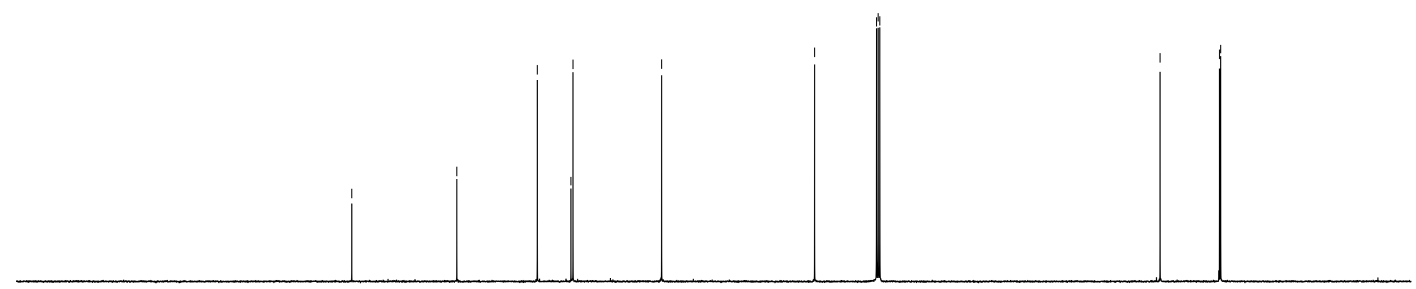

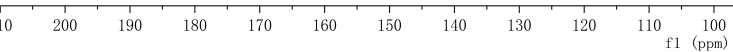




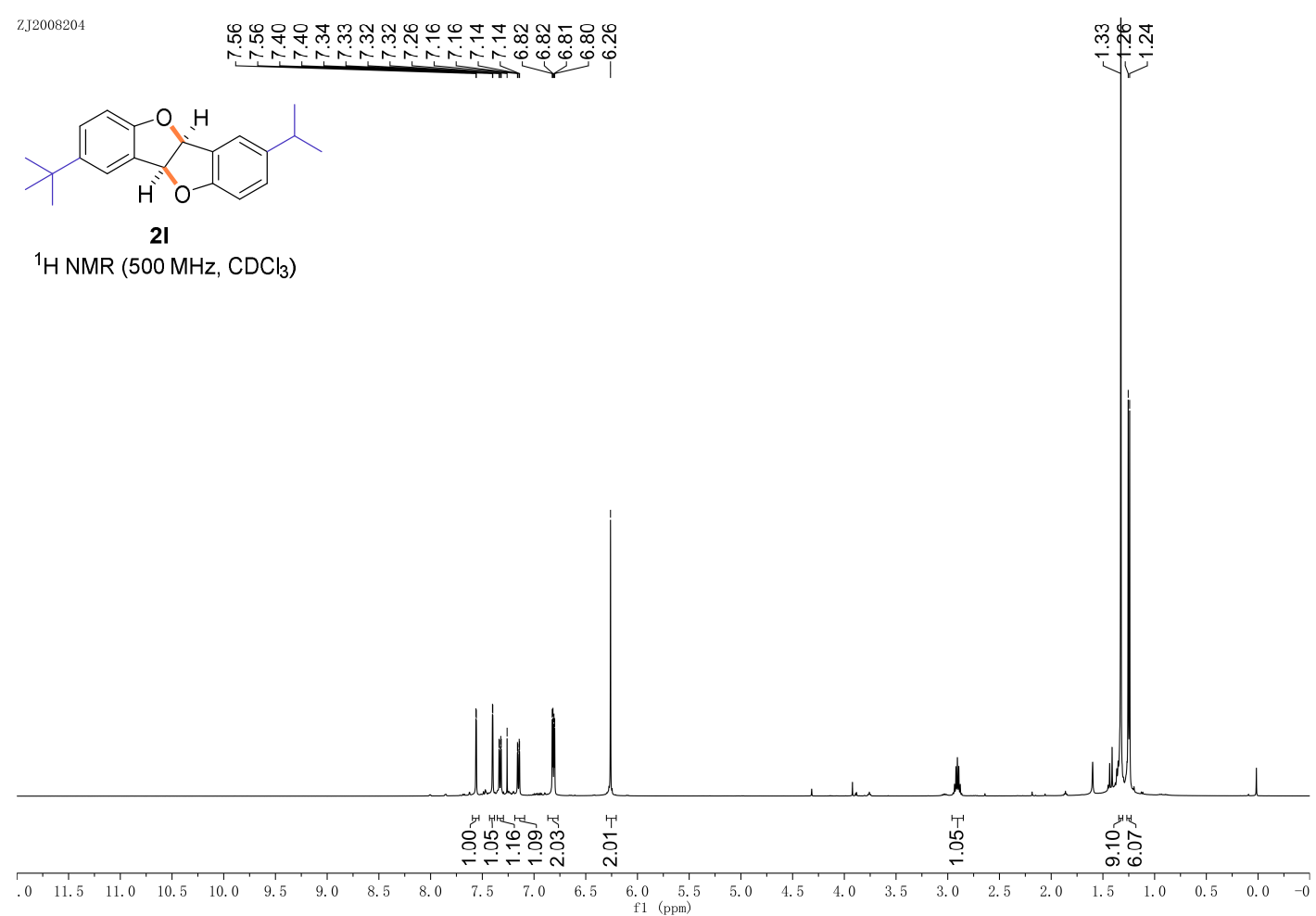

ZJ2008204
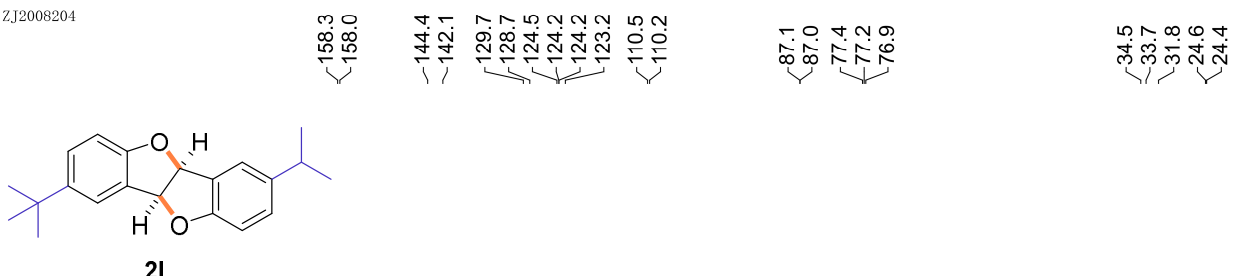

${ }^{13} \mathrm{C} \mathrm{NMR}\left(125 \mathrm{MHz}, \mathrm{CDCl}_{3}\right)$

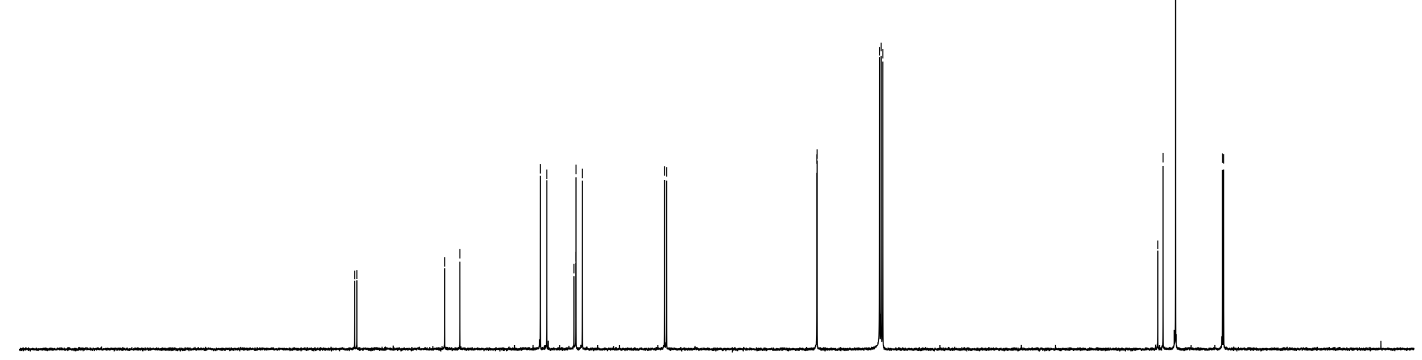

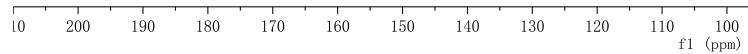




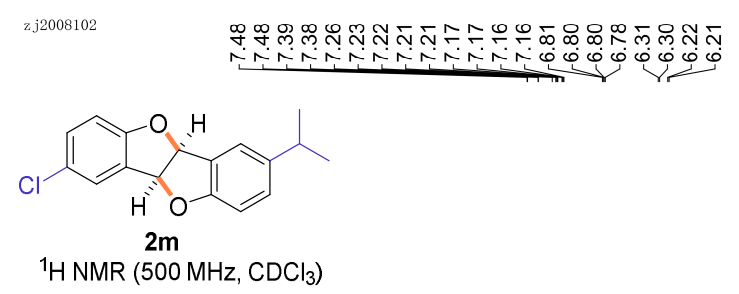

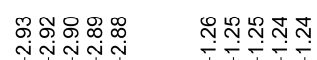

लูNนN

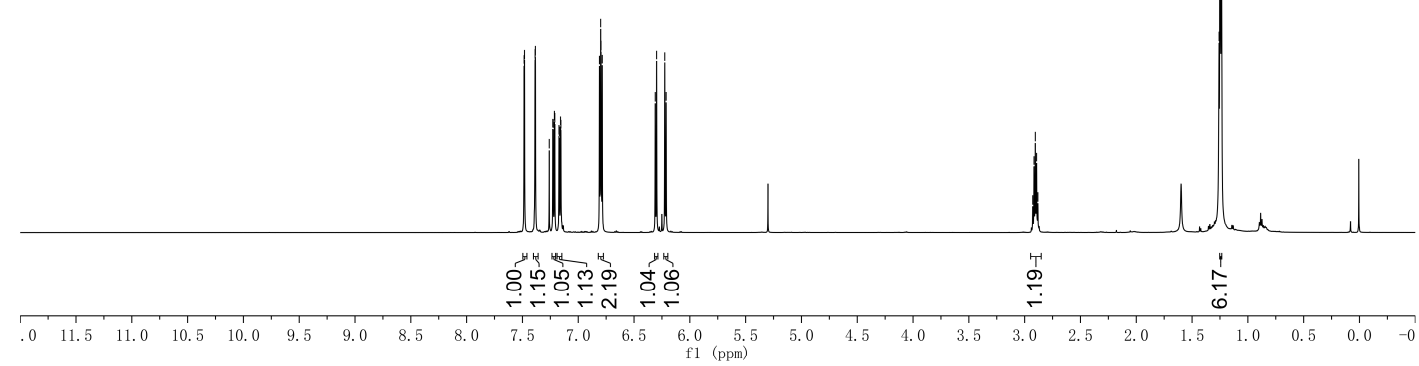

zj2008102
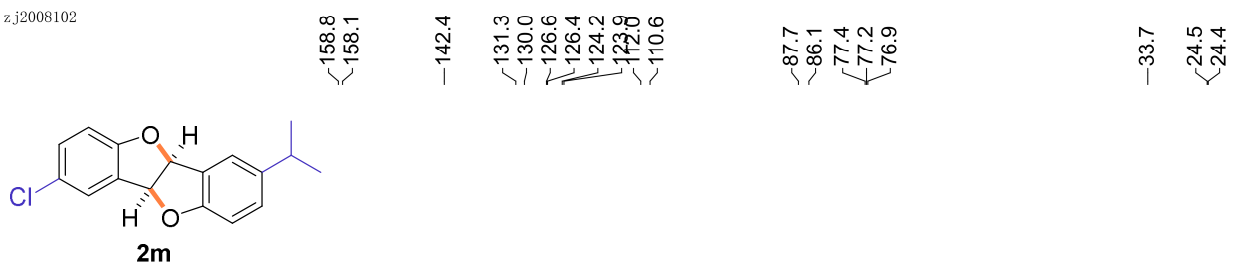

${ }^{13} \mathrm{C}$ NMR $\left(125 \mathrm{MHz}, \mathrm{CDCl}_{3}\right)$

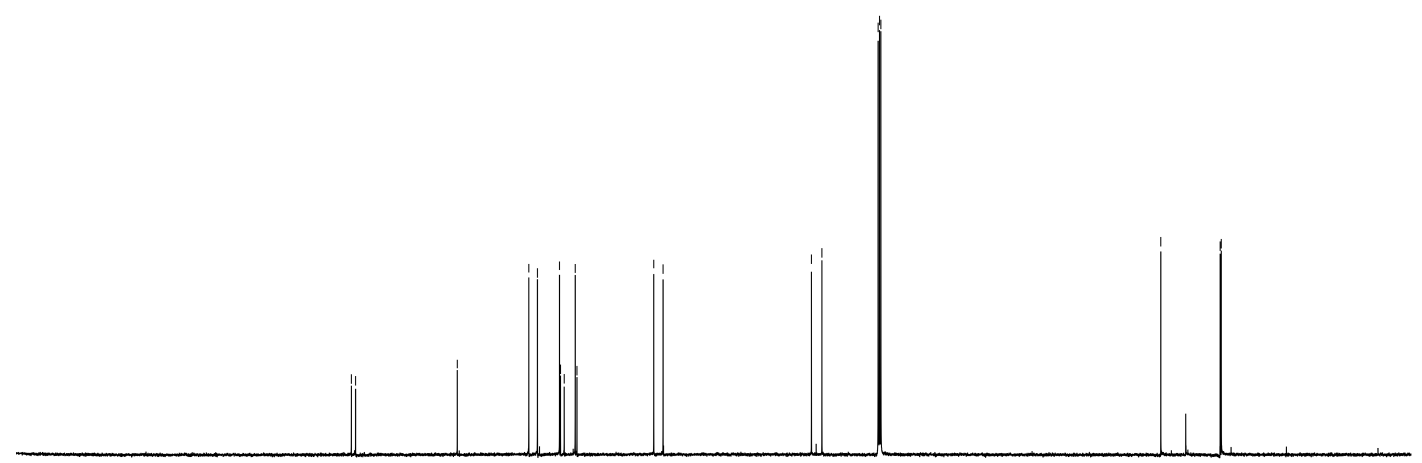

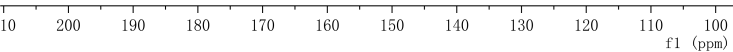




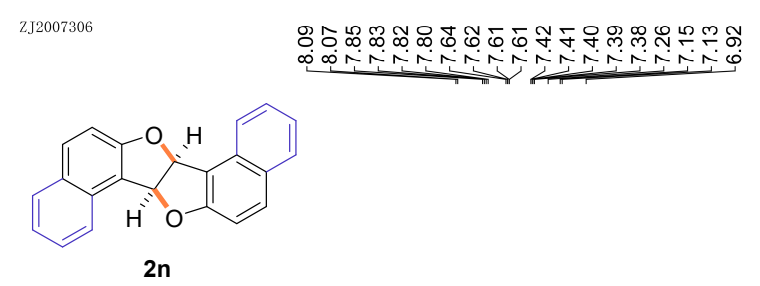

${ }^{1} \mathrm{H} \mathrm{NMR}\left(500 \mathrm{MHz}, \mathrm{CDCl}_{3}\right)$

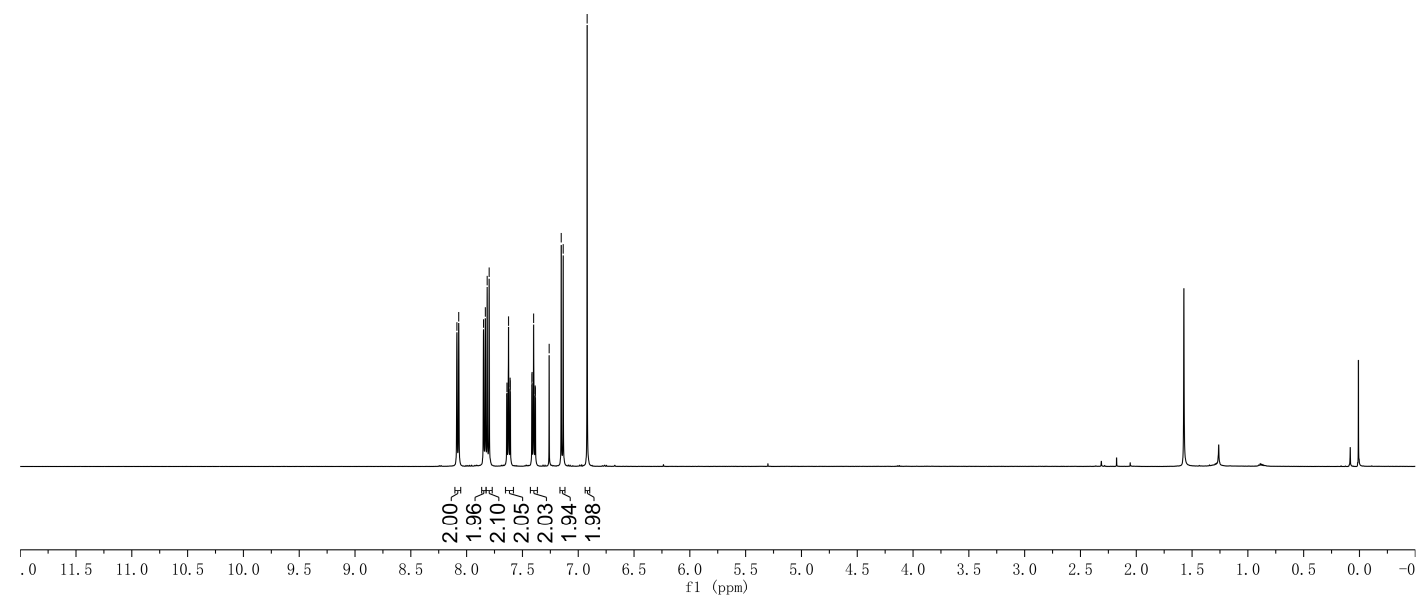

ZJ2007306
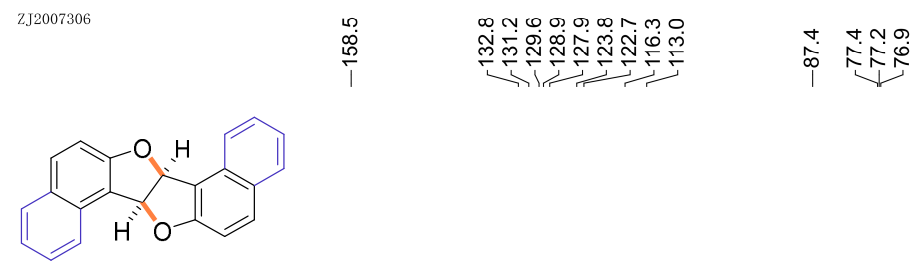

$2 \mathrm{n}$

${ }^{13} \mathrm{C} \mathrm{NMR}\left(125 \mathrm{MHz}, \mathrm{CDCl}_{3}\right)$

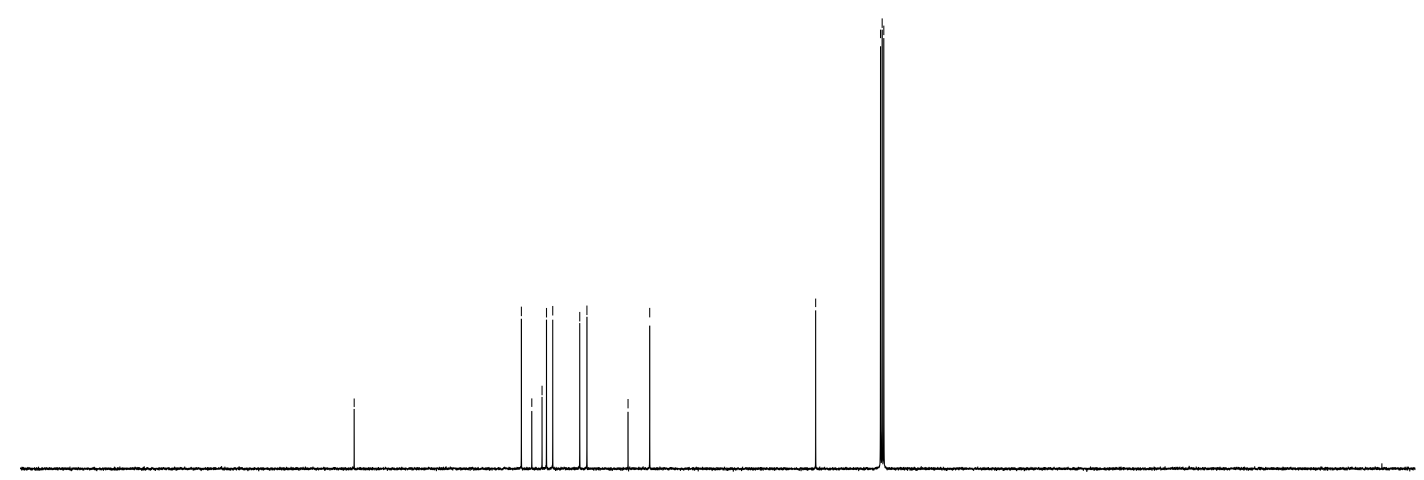

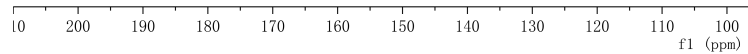




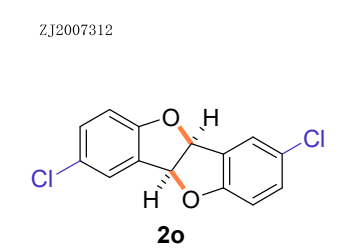

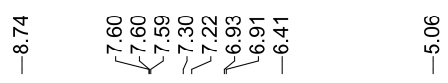

${ }^{1} \mathrm{H}$ NMR (500 MHz, Pyridine- $d_{5}$ )

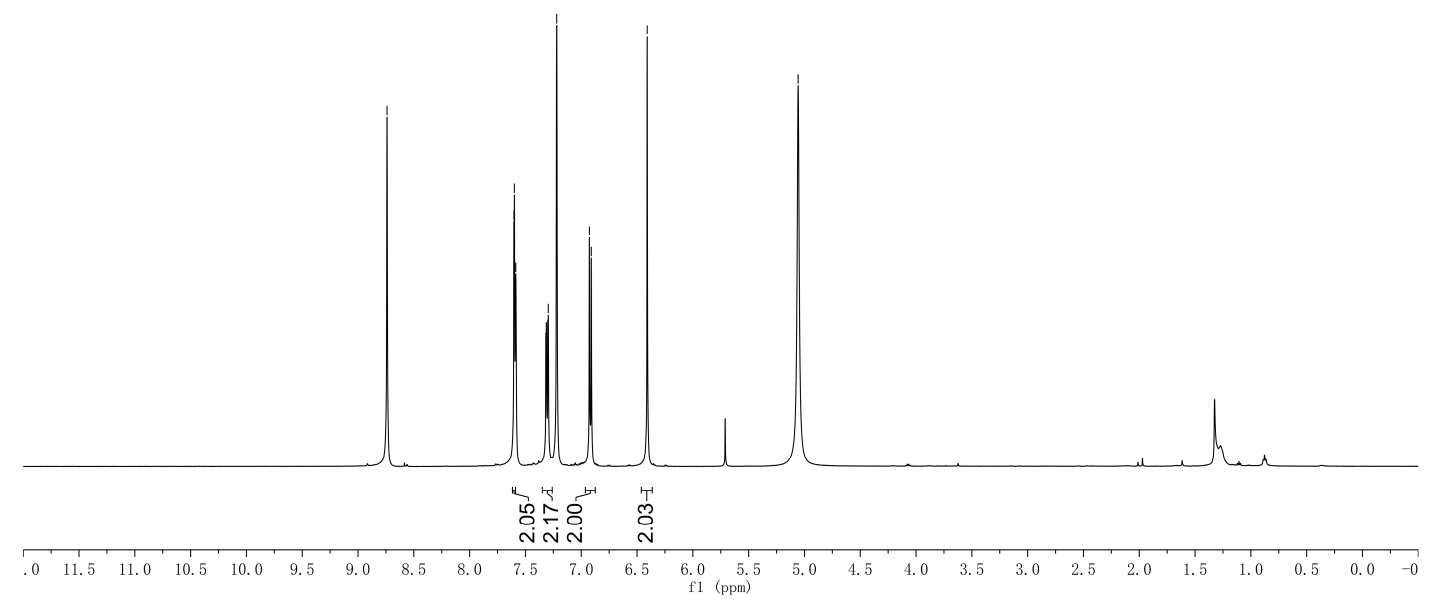

ZJ2007312

i.

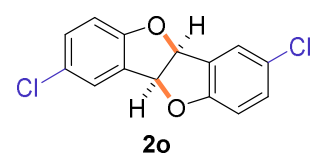

${ }^{13} \mathrm{C}$ NMR $(125 \mathrm{MHz}$, Pyridine-d $)$

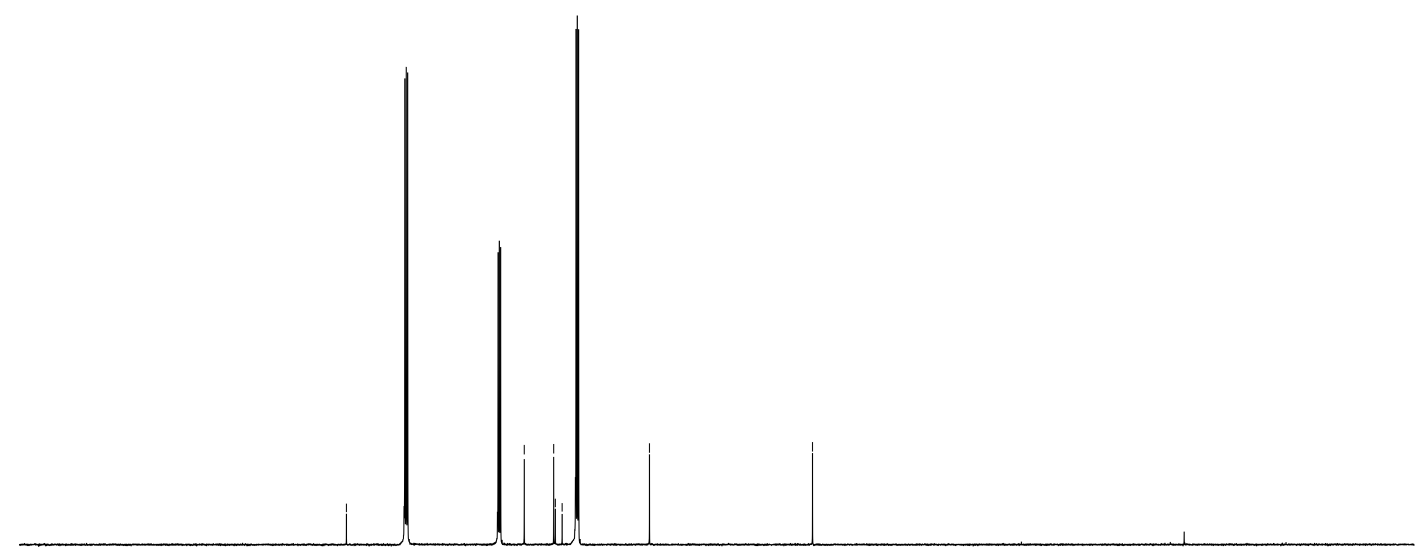

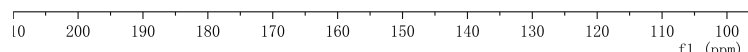




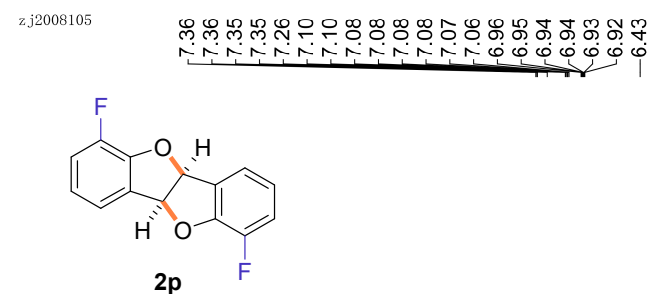

${ }^{1} \mathrm{H}$ NMR $\left(500 \mathrm{MHz}, \mathrm{CDCl}_{3}\right)$

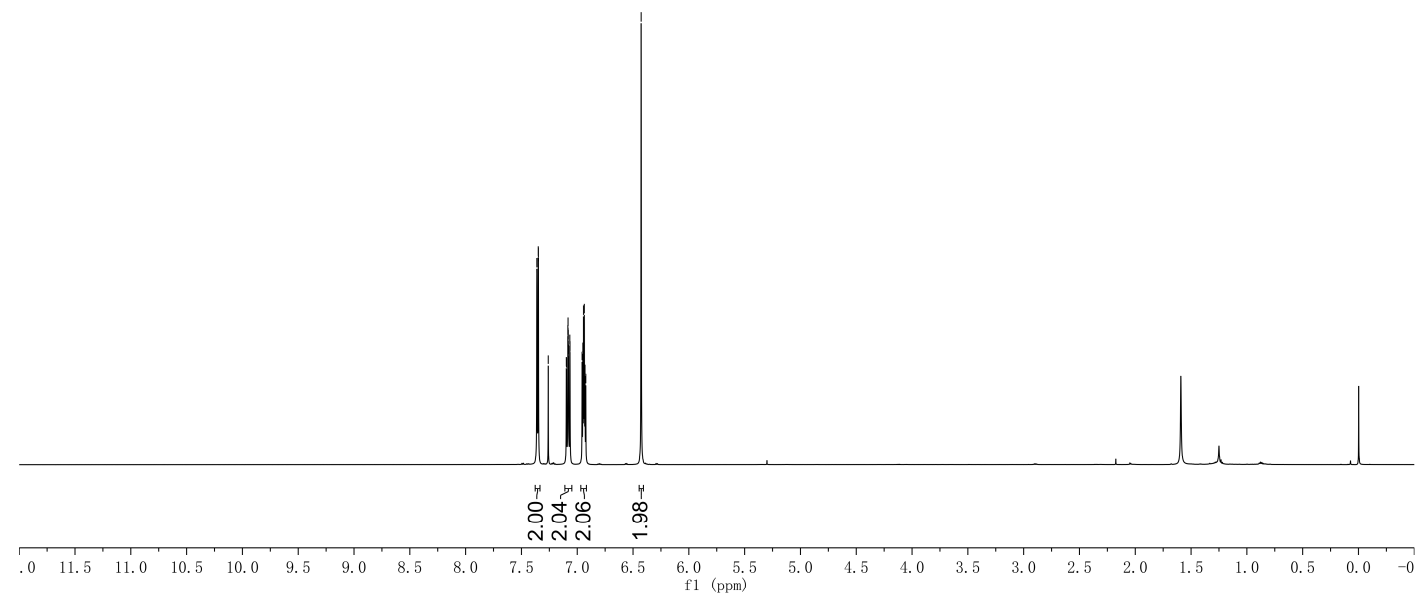

zj2008105

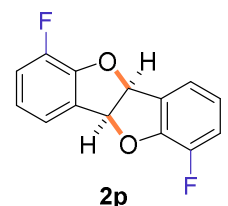

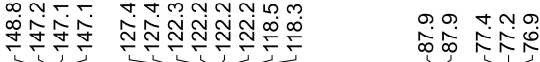

${ }^{13} \mathrm{CNMR}\left(125 \mathrm{MHz}, \mathrm{CDCl}_{3}\right)$

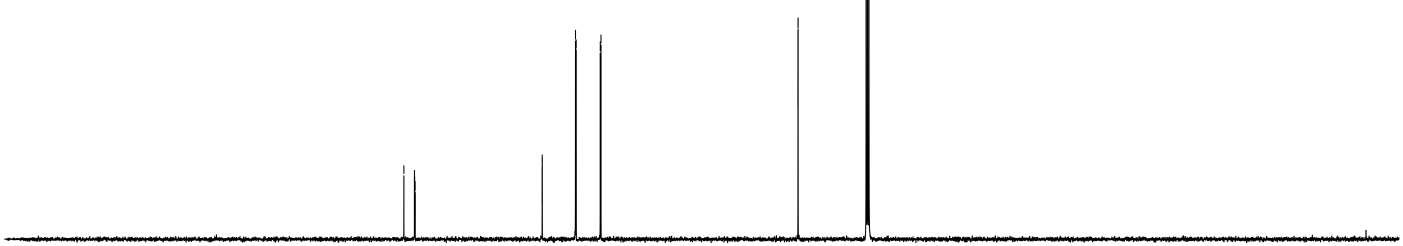

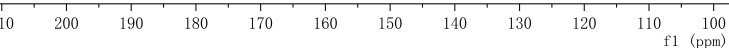


zj2008105F

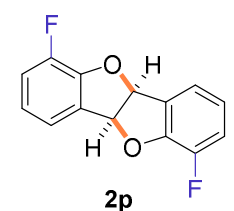

$\stackrel{\infty}{\substack{i \\ i}}$

${ }^{19} \mathrm{~F}$ NMR (471 MHz, $\left.\mathrm{CDCl}_{3}\right)$

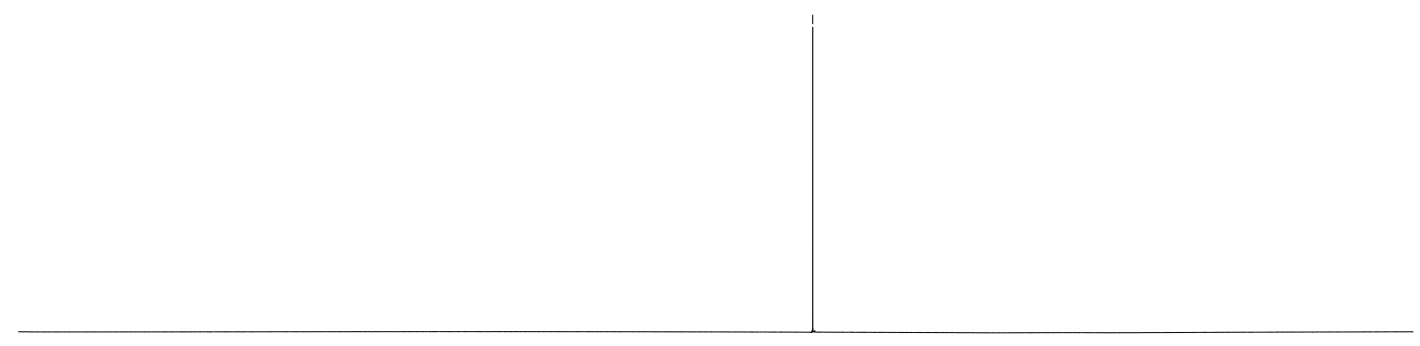

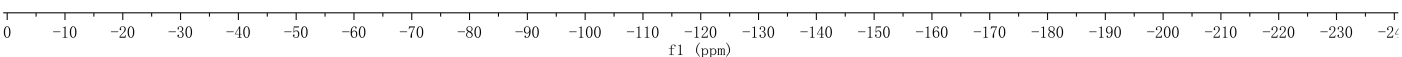




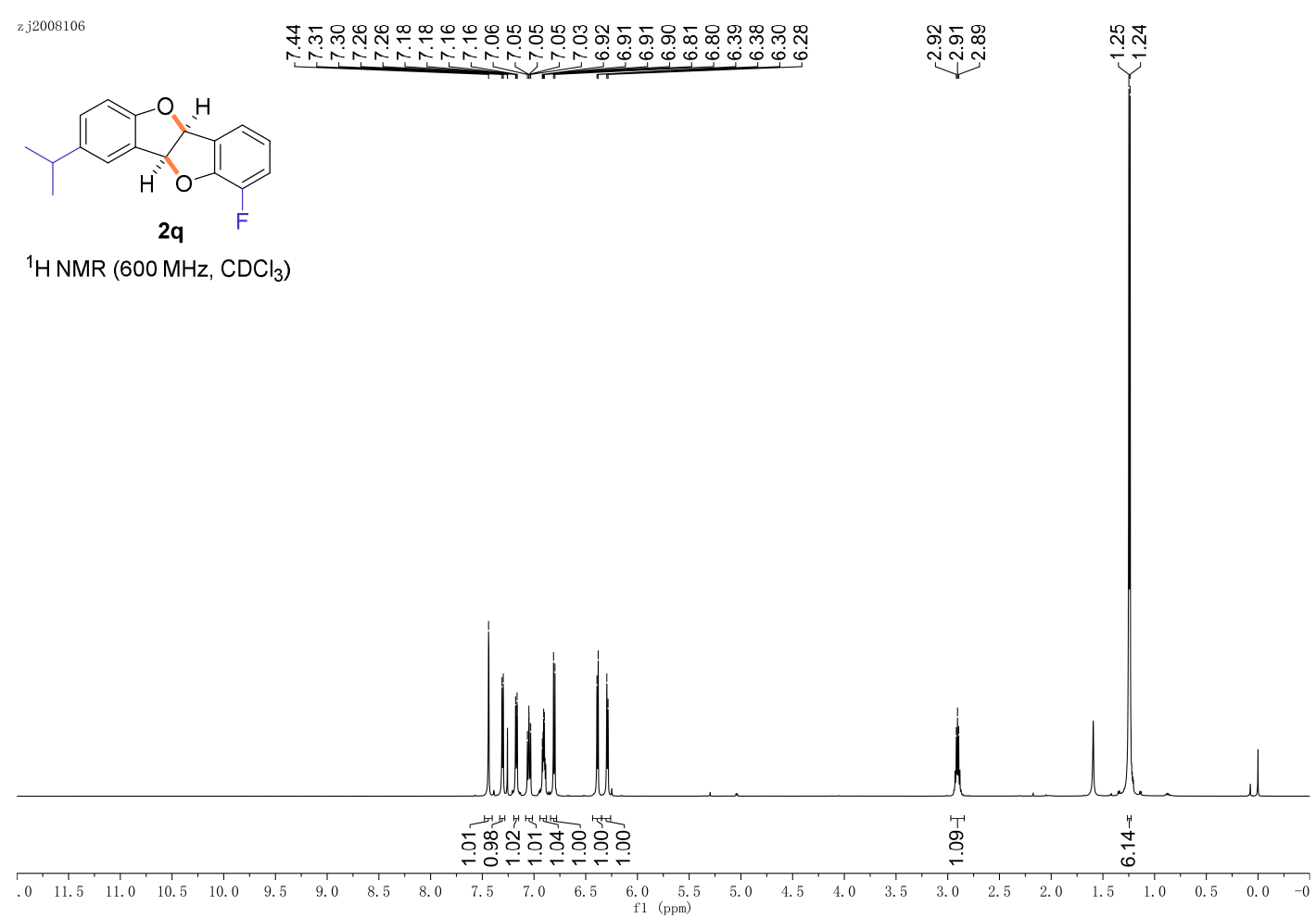

zj2008106

\section{至}

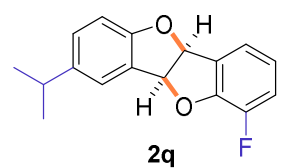

${ }^{13} \mathrm{C}$ NMR $\left(150 \mathrm{MHz}, \mathrm{CDCl}_{3}\right)$

C NIMR $\left(150 \mathrm{MHz}_{2}, \mathrm{CDCl}_{3}\right)$

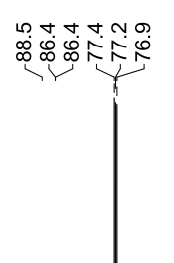

皇

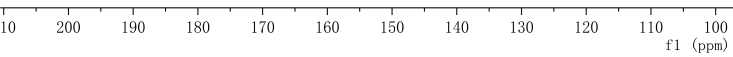


zj2008106F

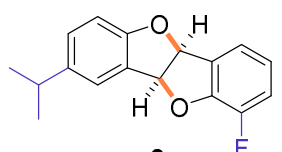

2q

${ }^{19} \mathrm{~F} \mathrm{NMR}\left(471 \mathrm{MHz}, \mathrm{CDCl}_{3}\right)$

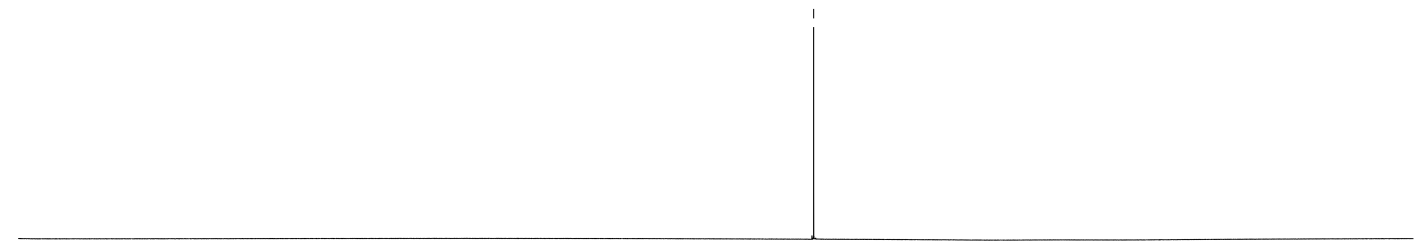

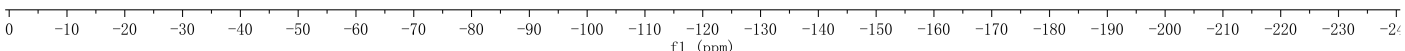




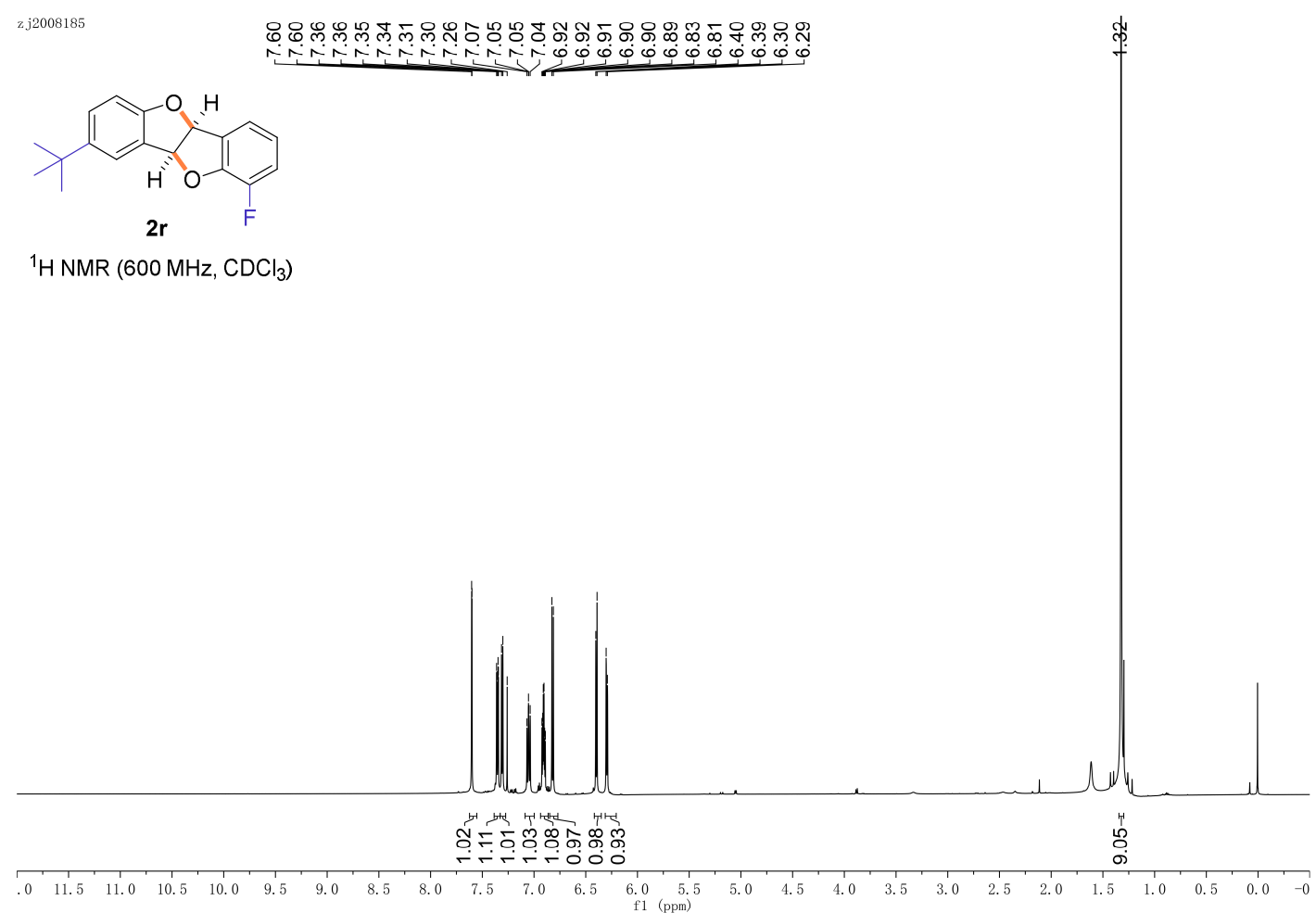

zj2008185

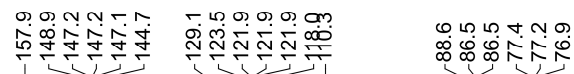

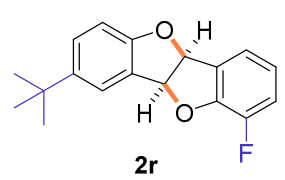

${ }^{13} \mathrm{C}$ NMR $\left(150 \mathrm{MHz}, \mathrm{CDCl}_{3}\right)$

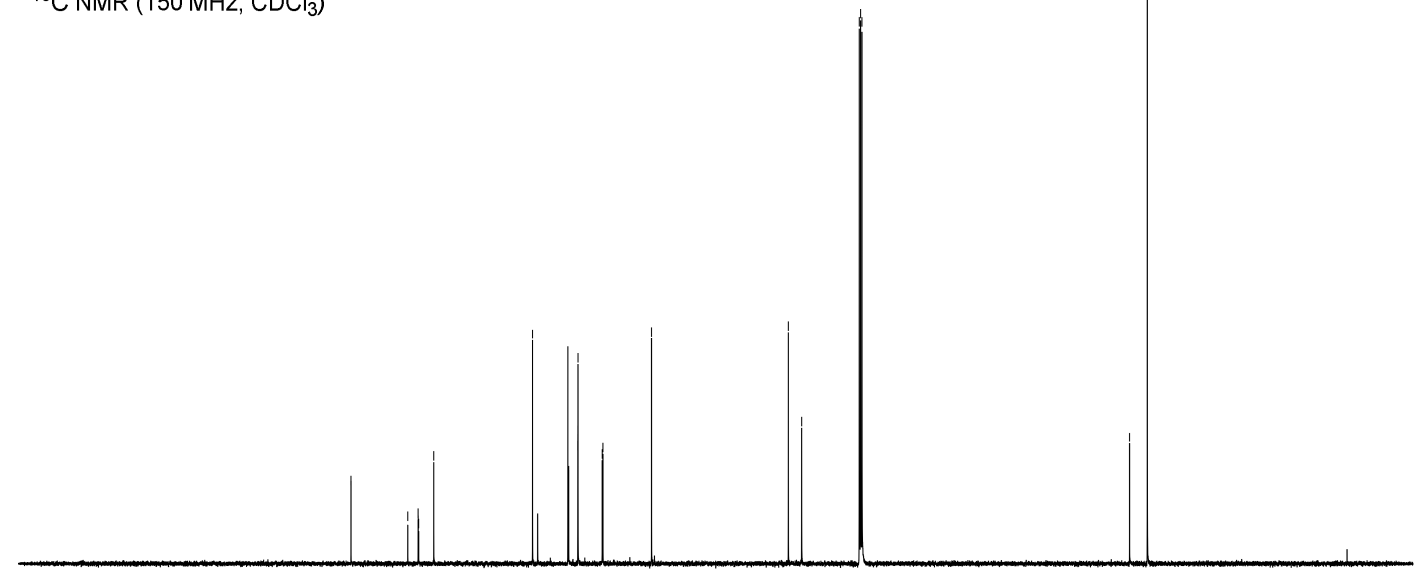

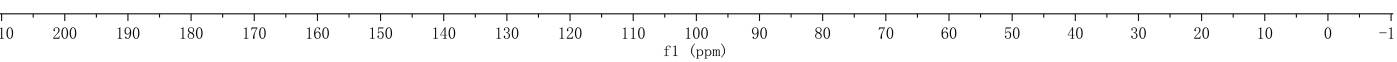


zj2008185F

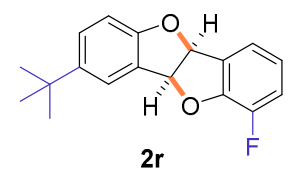

0
0
$\stackrel{0}{i}$

${ }^{19} \mathrm{~F} \mathrm{NMR}\left(471 \mathrm{MHz}, \mathrm{CDCl}_{3}\right)$

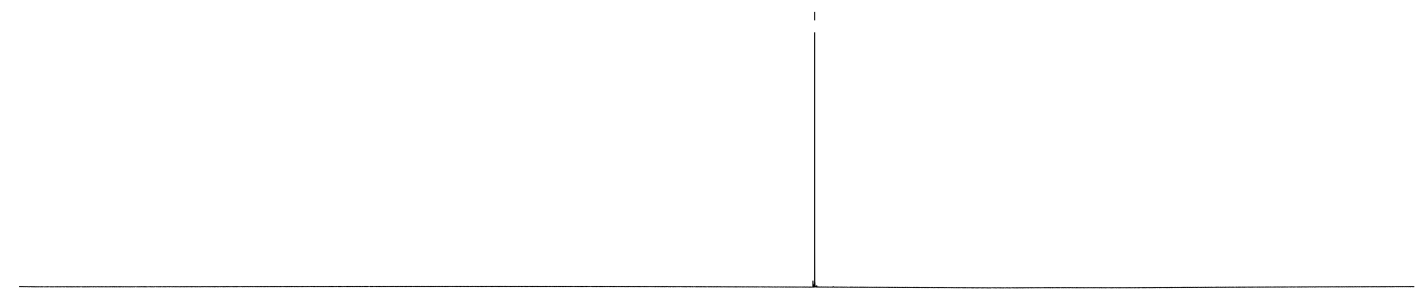

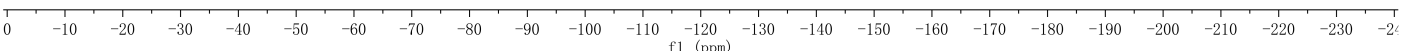




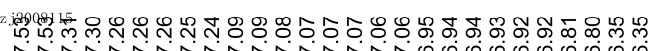

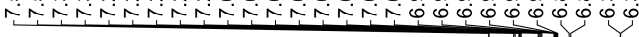

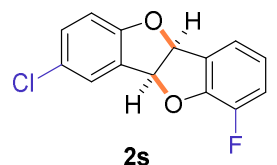

${ }^{1} \mathrm{H} \mathrm{NMR}\left(600 \mathrm{MHz}, \mathrm{CDCl}_{3}\right)$

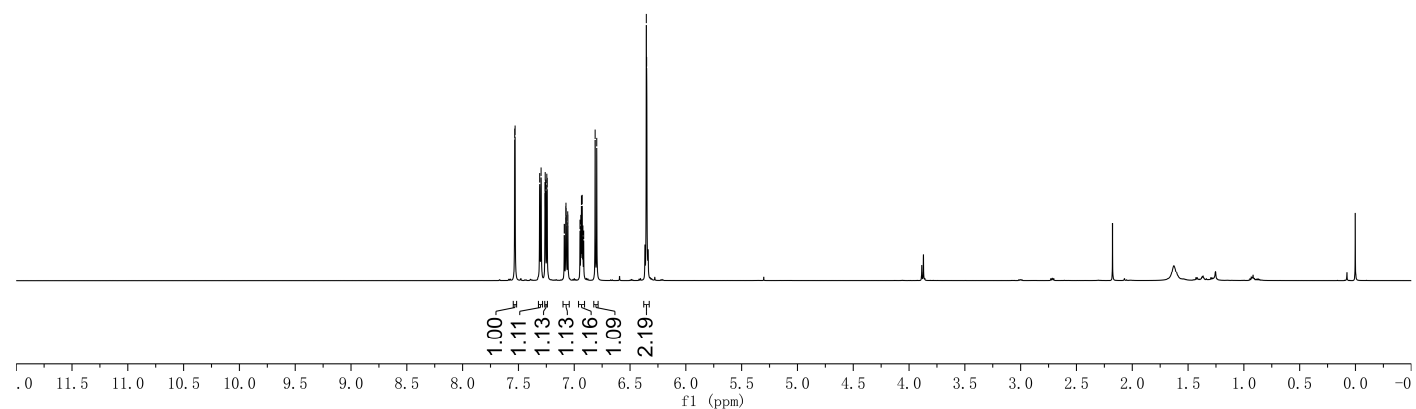

zj2008115
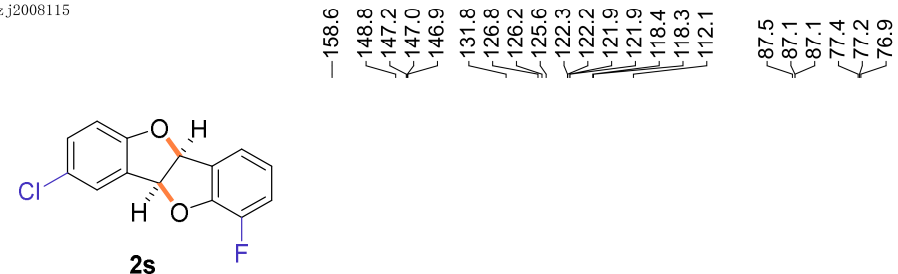

${ }^{13} \mathrm{C} \mathrm{NMR}\left(150 \mathrm{MHz}, \mathrm{CDCl}_{3}\right)$

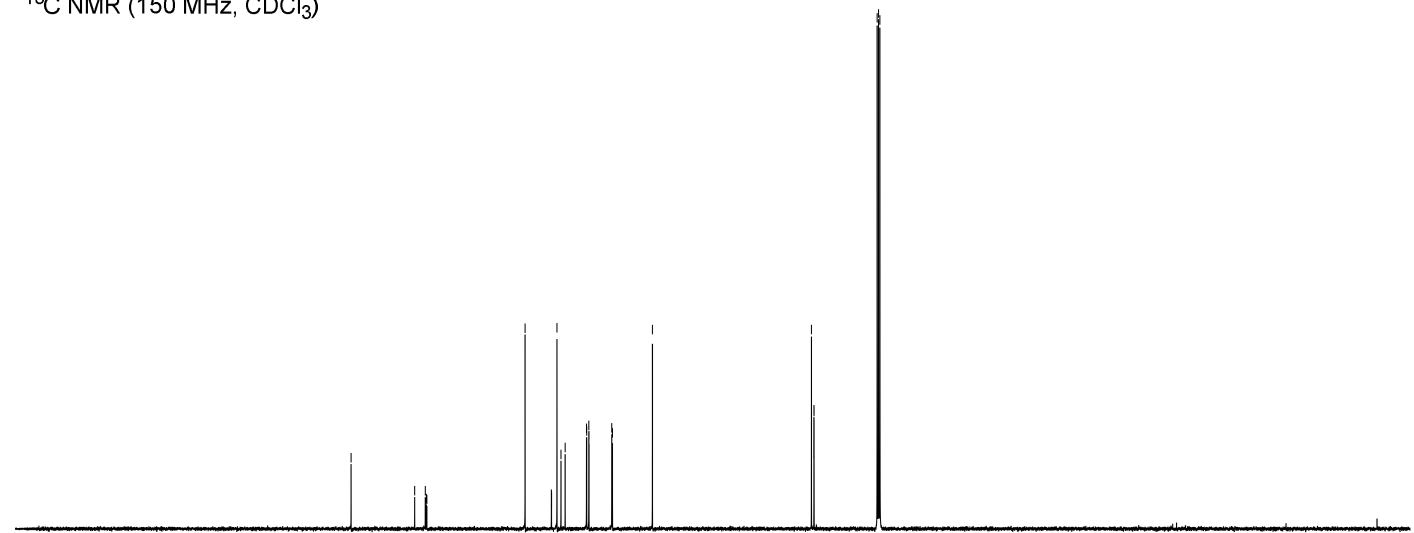

$\begin{array}{lllllllllllll}10 & 200 & 190 & 180 & 170 & 160 & 150 & 140 & 130 & 120 & 110 & 1 \\ \mathrm{f} 1(\mathrm{pmm}) & 1\end{array}$ 
zj2008115F

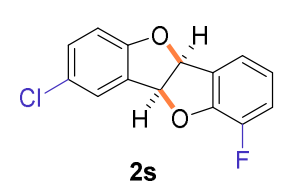

$\stackrel{\leftrightarrow}{\stackrel{0}{i}}$

$\left.{ }^{19} \mathrm{~F} \mathrm{NMR} \mathrm{(471} \mathrm{MHz,} \mathrm{CDCl}_{3}\right)$

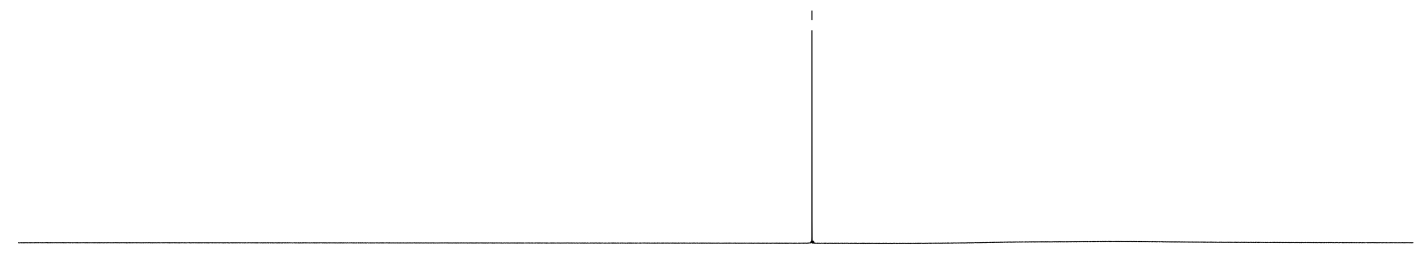

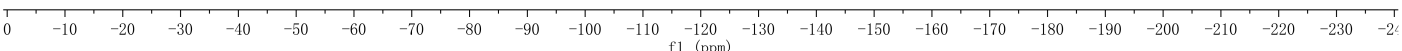



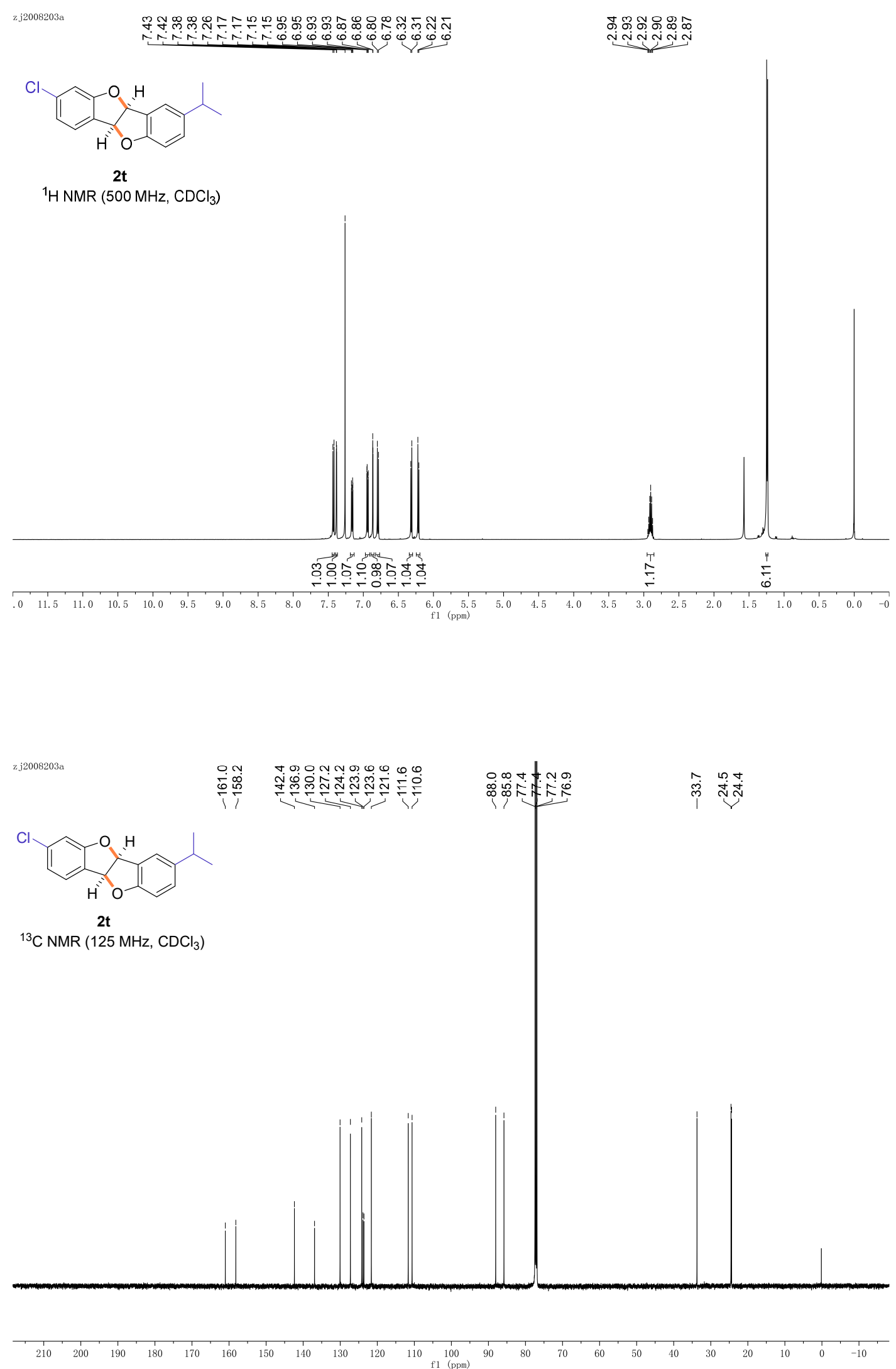


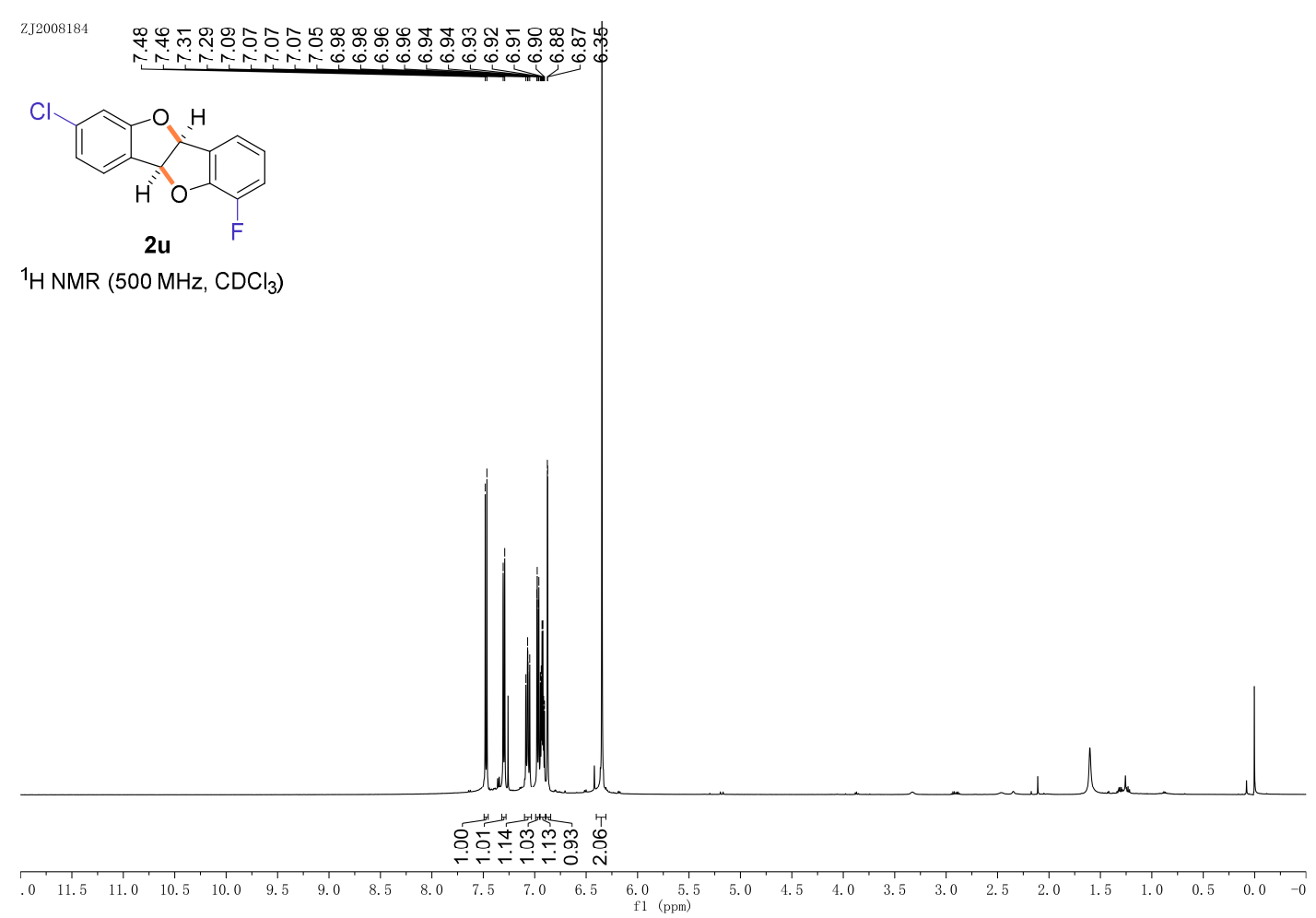

2J2008184

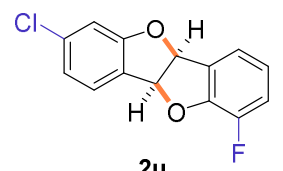

${ }^{13} \mathrm{C} \mathrm{NMR}\left(125 \mathrm{MHz}, \mathrm{CDCl}_{3}\right)$

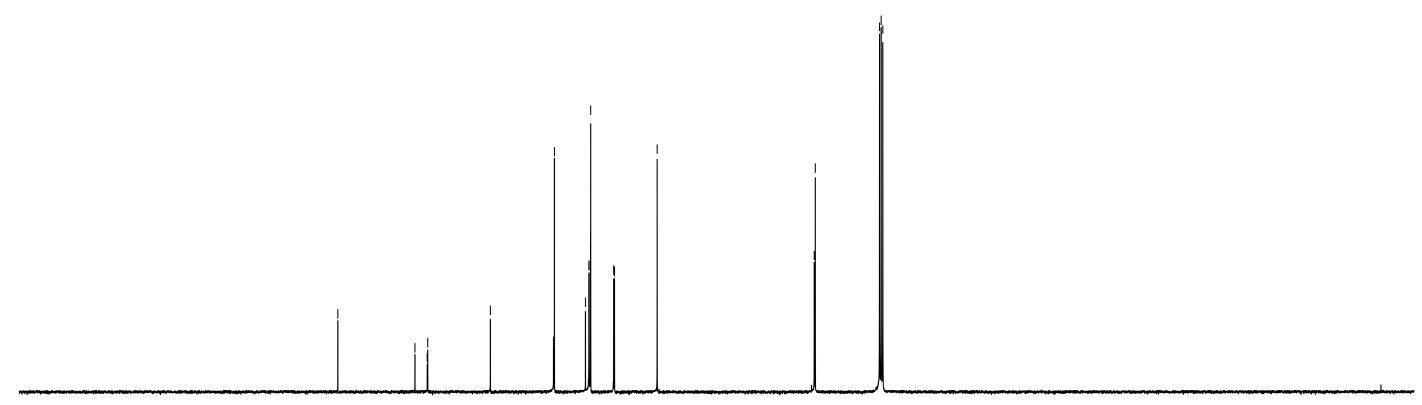

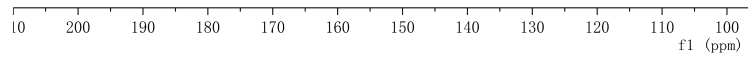




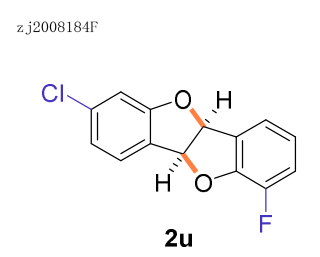

${ }^{19} \mathrm{~F} \mathrm{NMR}\left(471 \mathrm{MHz}, \mathrm{CDCl}_{3}\right)$

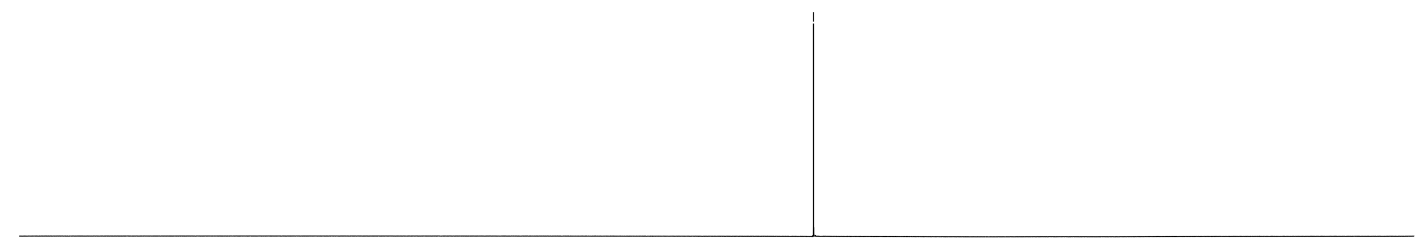

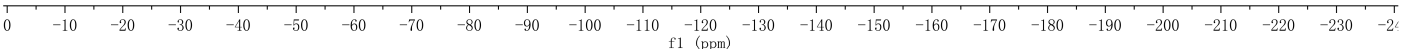




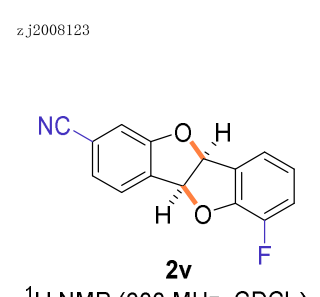

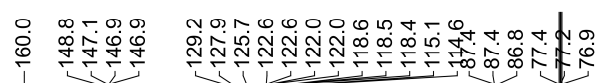

${ }^{1} \mathrm{H}$ NMR $\left(600 \mathrm{MHz}, \mathrm{CDCl}_{3}\right)$
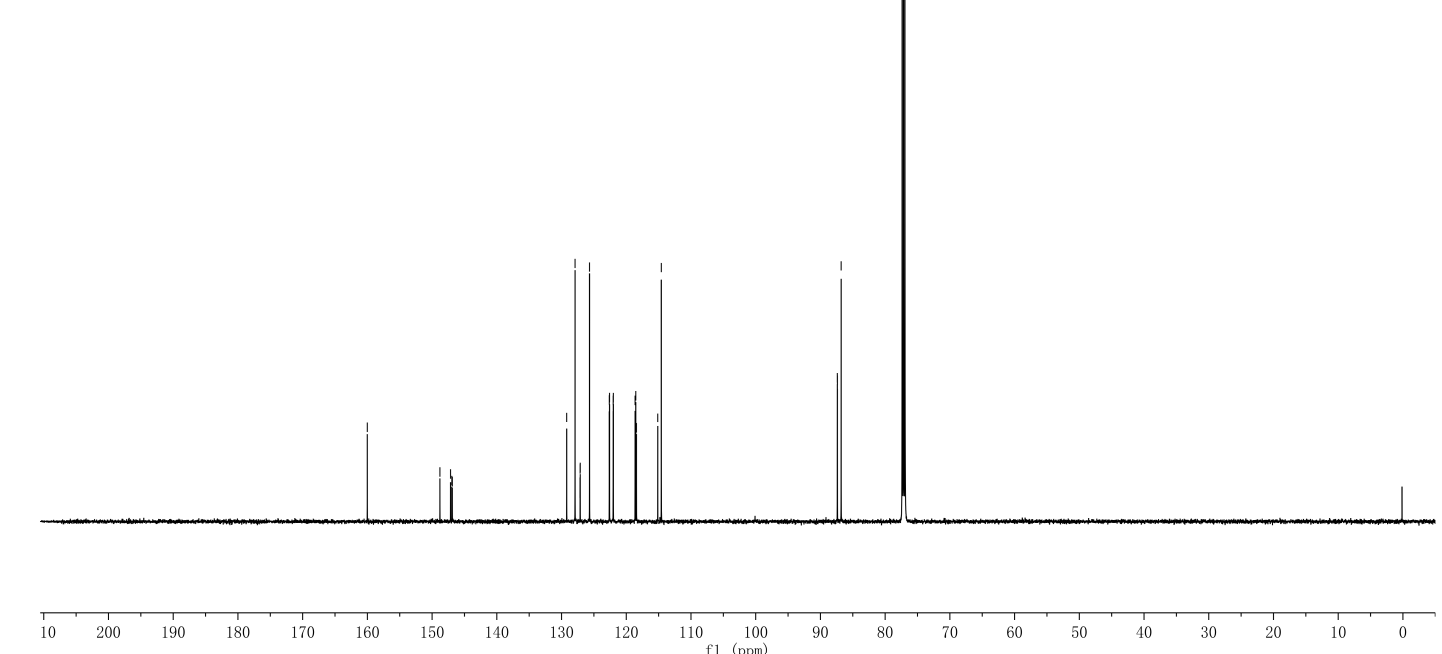

z j2008123

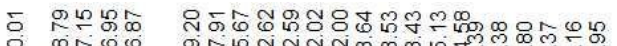

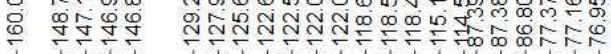

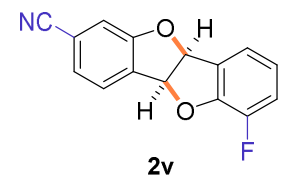

$\left.{ }^{13} \mathrm{C} \mathrm{NMR} \mathrm{(150MHz,} \mathrm{CDCl}_{3}\right)$

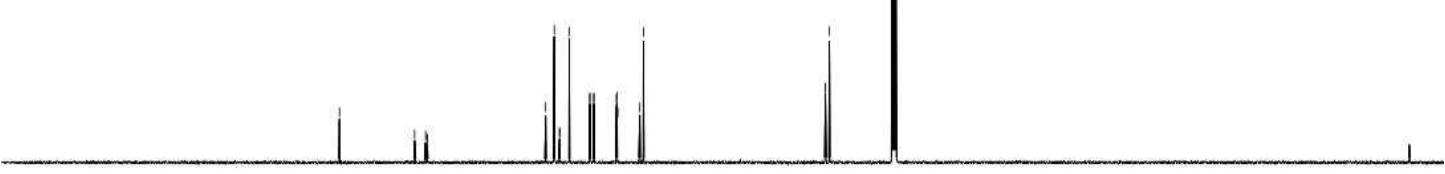

\begin{tabular}{llllllllllllll}
\hline 10 & 200 & 190 & 180 & 170 & 160 & 150 & 140 & 130 & 120 & 110 & 100 \\
\hline
\end{tabular} 


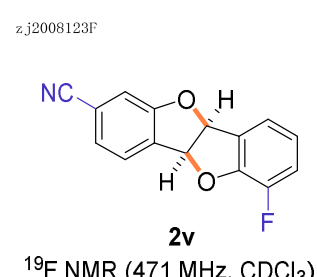

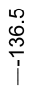

${ }^{19} \mathrm{~F} \mathrm{NMR}\left(471 \mathrm{MHz}, \mathrm{CDCl}_{3}\right)$

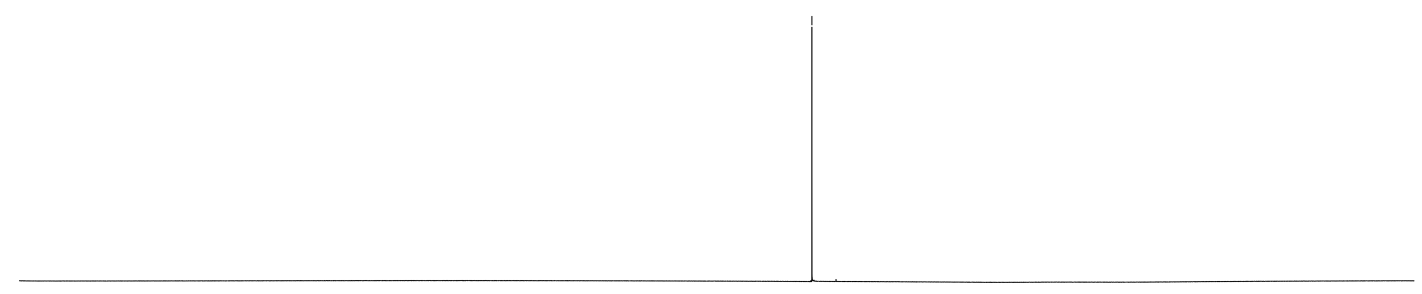

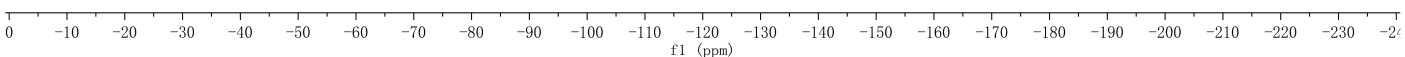



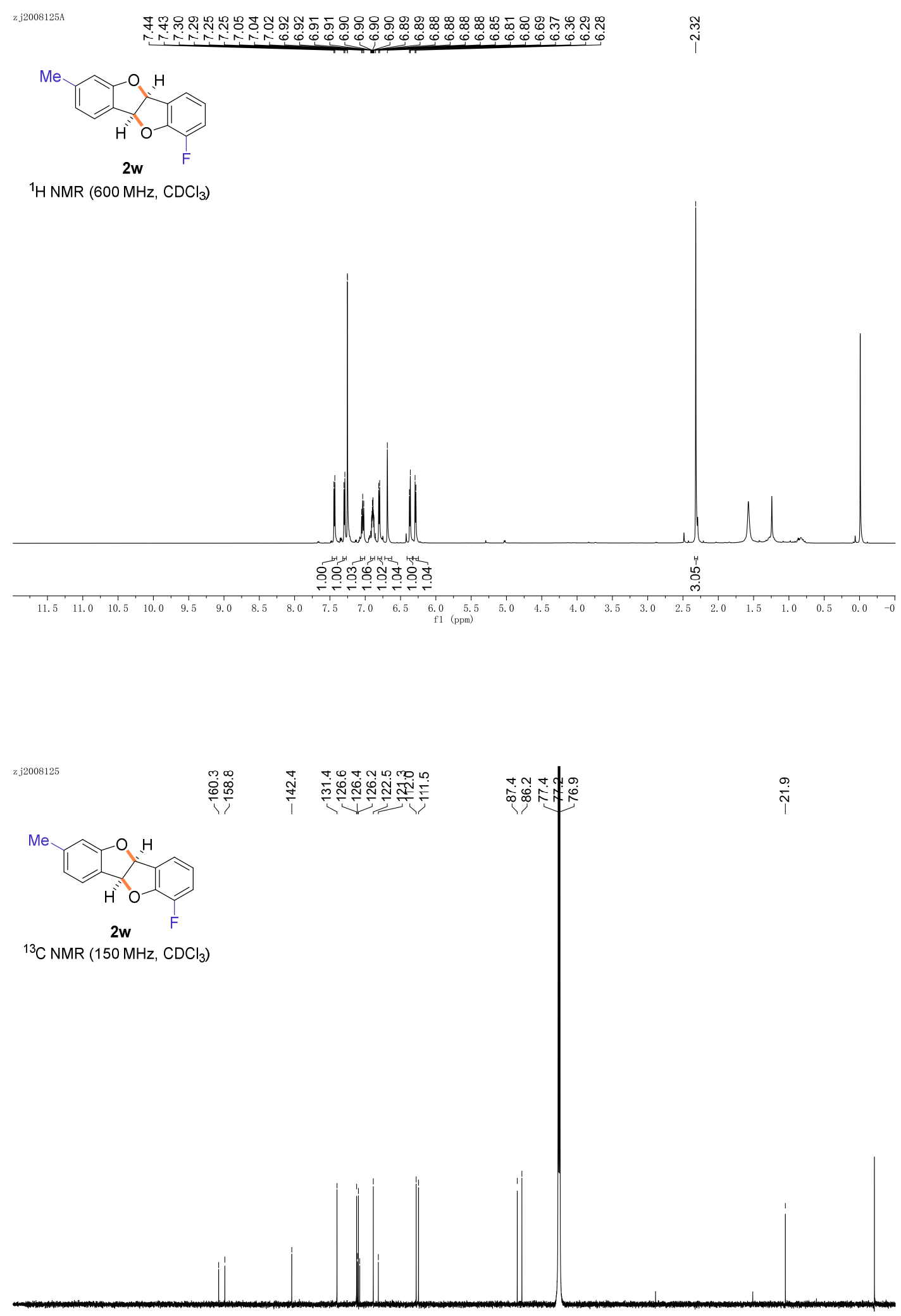

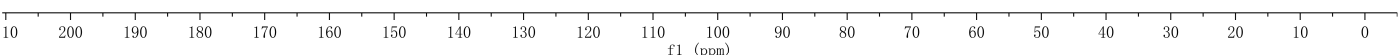




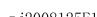<smiles>Cc1ccc2c(c1)O[C@H]1c3cccc(F)c3O[C@H]21</smiles>

${ }^{19} \mathrm{~F}$ NMR $\left(471 \mathrm{MHz}, \mathrm{CDCl}_{3}\right)$

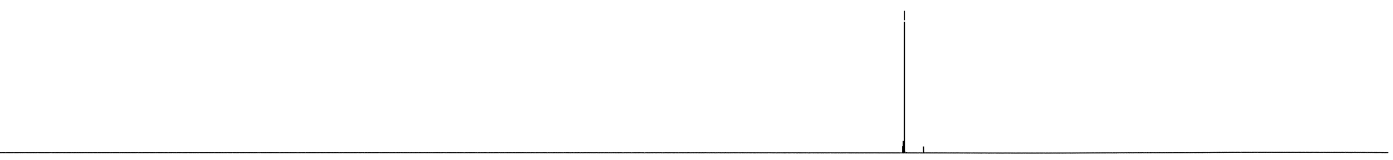

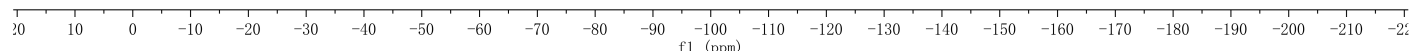



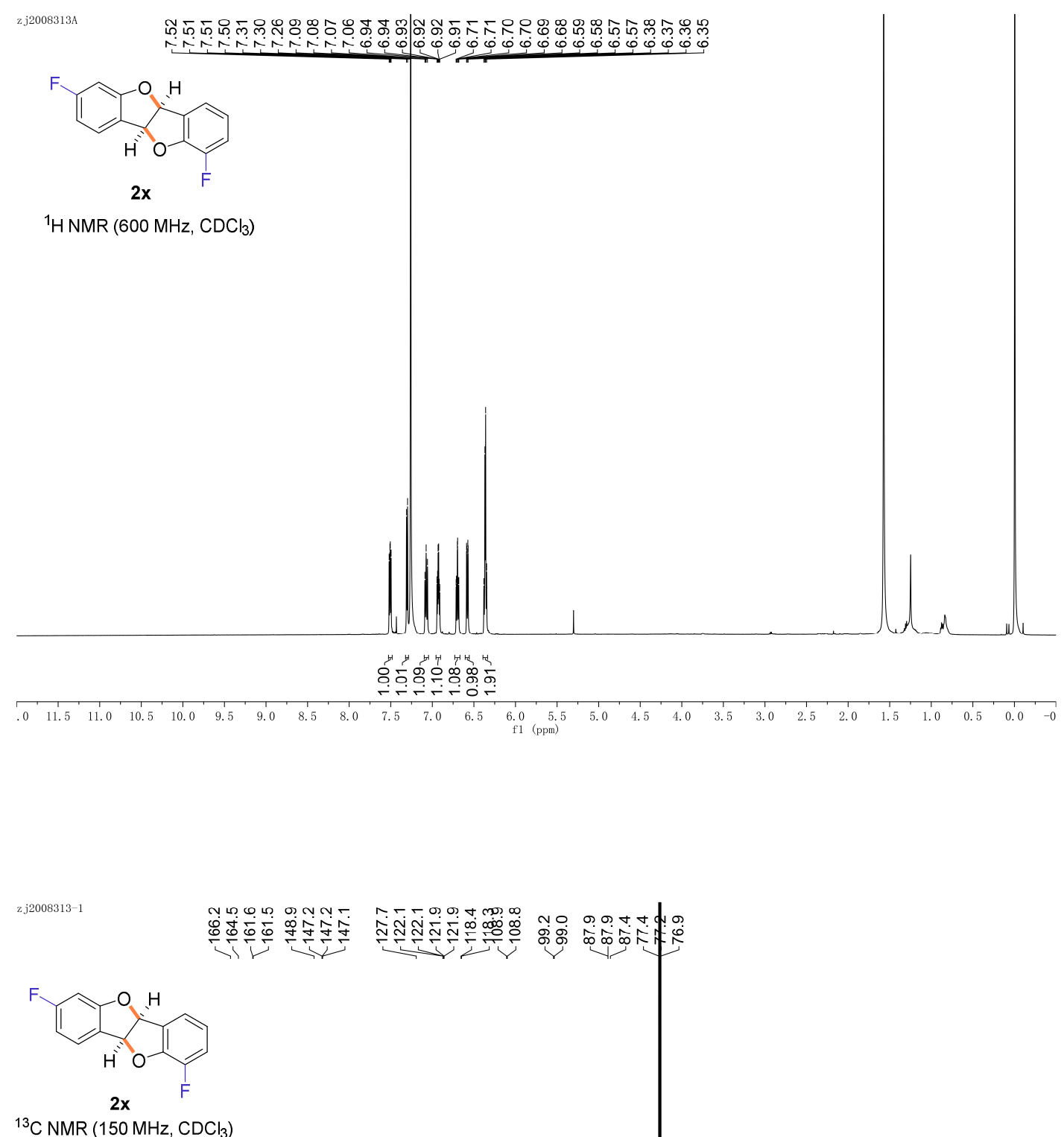

${ }^{13} \mathrm{C} \mathrm{NMR}\left(150 \mathrm{MHz}, \mathrm{CDCl}_{3}\right)$ 


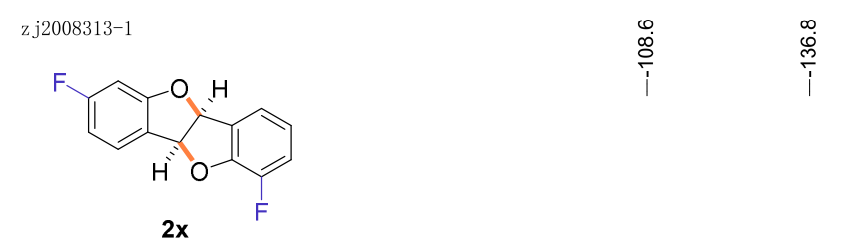

${ }^{19} \mathrm{~F} \mathrm{NMR}\left(471 \mathrm{MHz}, \mathrm{CDCl}_{3}\right)$

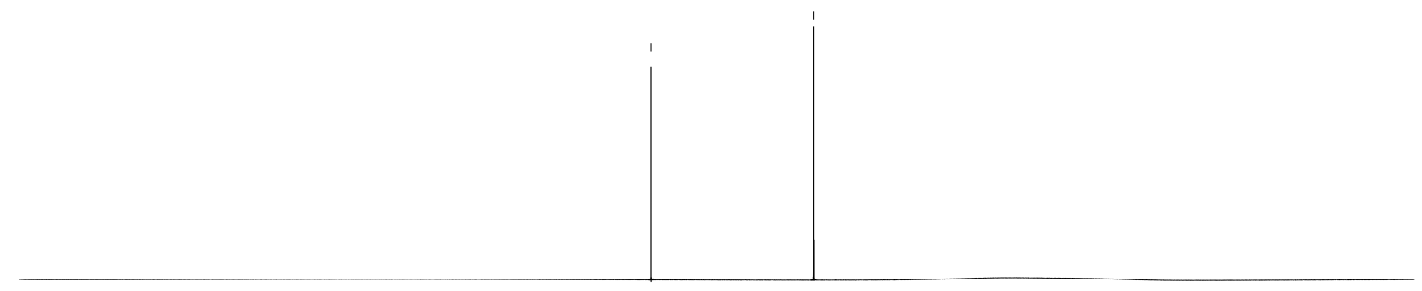

\begin{tabular}{llllllllllllllllllllllllllllll}
\hline & 1 & -10 & -20 & -30 & -40 & -50 & -60 & -70 & -80 & -90 & -100 & -110 & -120 & -130 & -140 & -150 & -160 & -170 & -180 & -190 & -200 & -210 & -220 & -230 & -2 \\
\hline
\end{tabular} 


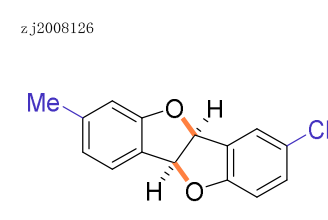

$2 y$

${ }^{1} \mathrm{H} \mathrm{NMR}\left(600 \mathrm{MHz}, \mathrm{CDCl}_{3}\right)$

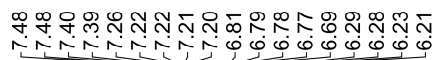

$\stackrel{\text { m }}{\text { in }}$

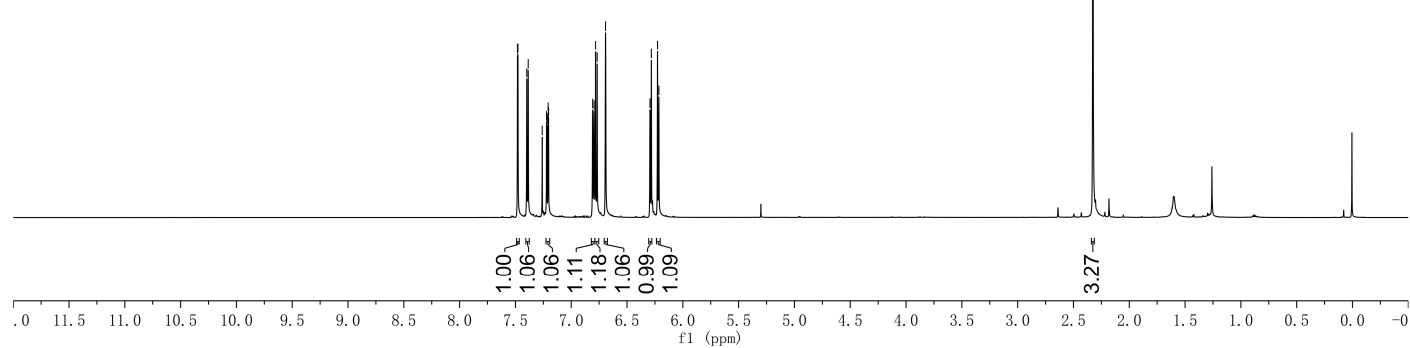

zj2008126

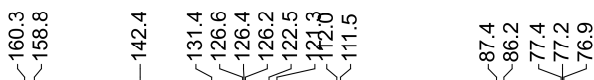

$\stackrel{\infty}{\grave{N}}$<smiles>Cc1ccc2c(c1)O[C@H]1c3cc(Cl)ccc3O[C@H]21</smiles>

2y

${ }^{13} \mathrm{C}$ NMR $\left(150 \mathrm{MHz}, \mathrm{CDCl}_{3}\right)$

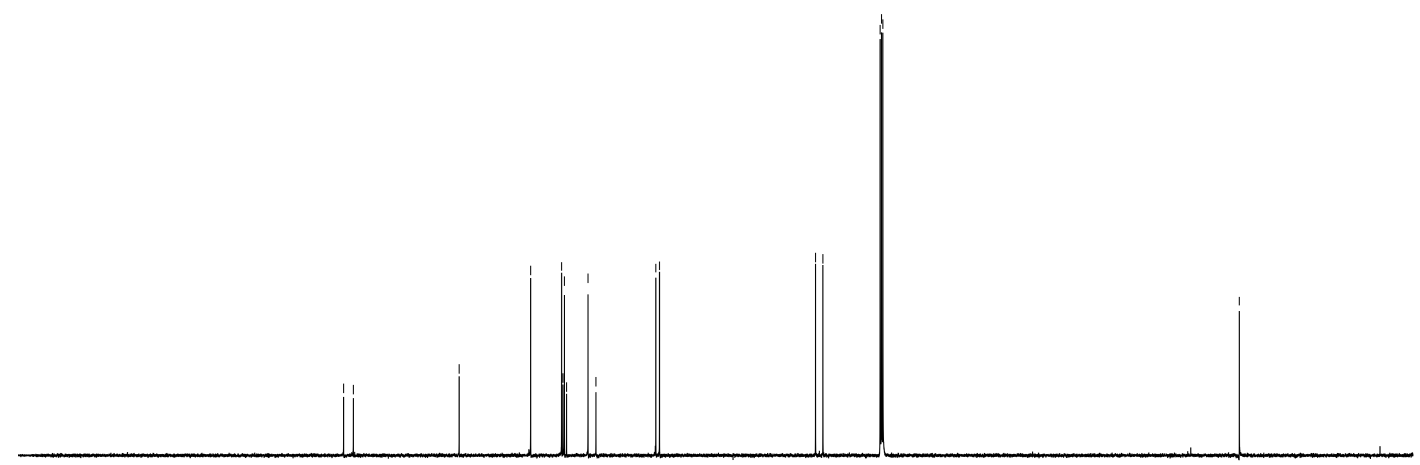

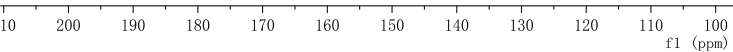




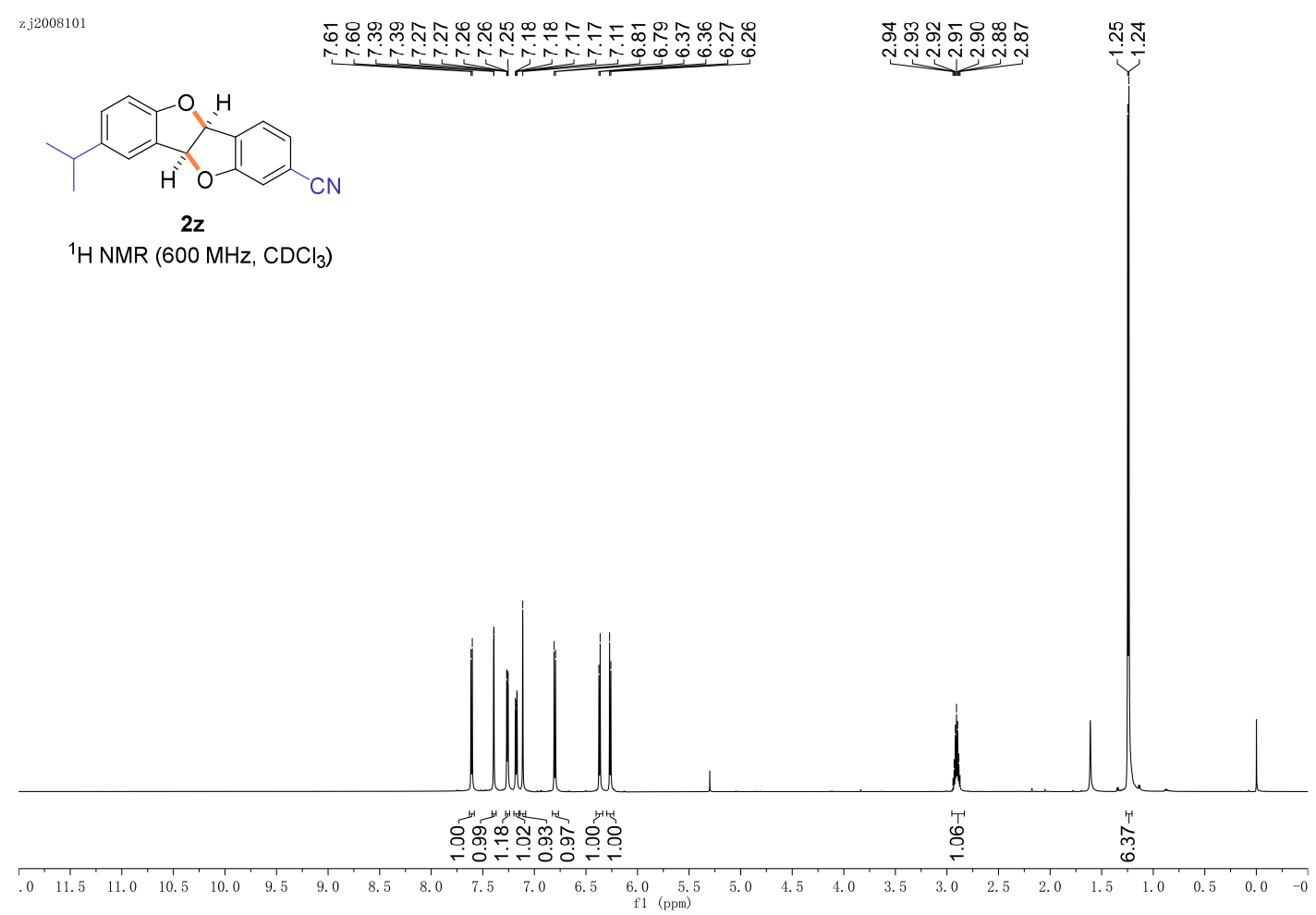

zj300101

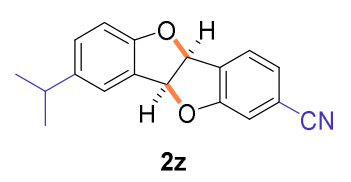

${ }^{13} \mathrm{C} \mathrm{NMR}\left(150 \mathrm{MHz}, \mathrm{CDCl}_{3}\right)$

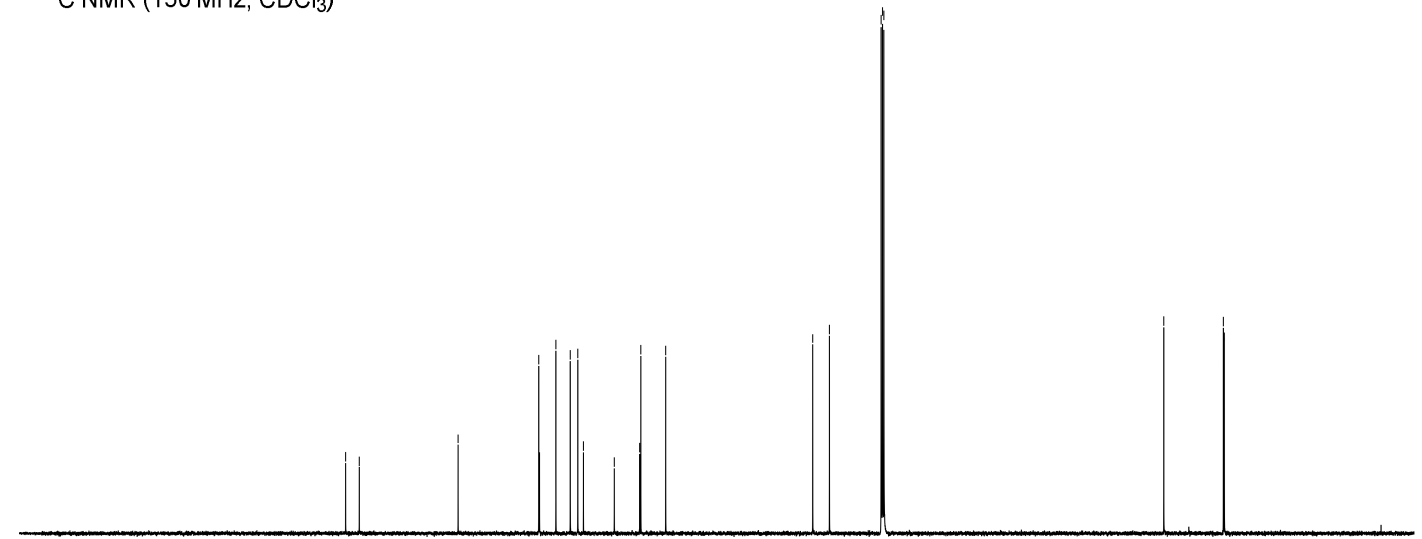

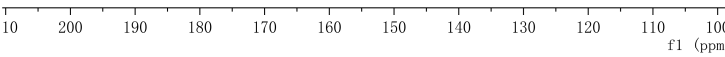




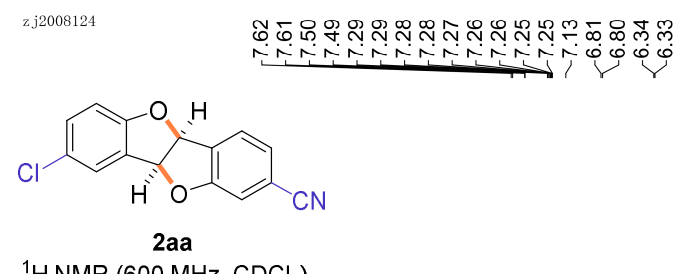

${ }^{1} \mathrm{HNMR}\left(600 \mathrm{MHz}, \mathrm{CDCl}_{3}\right)$

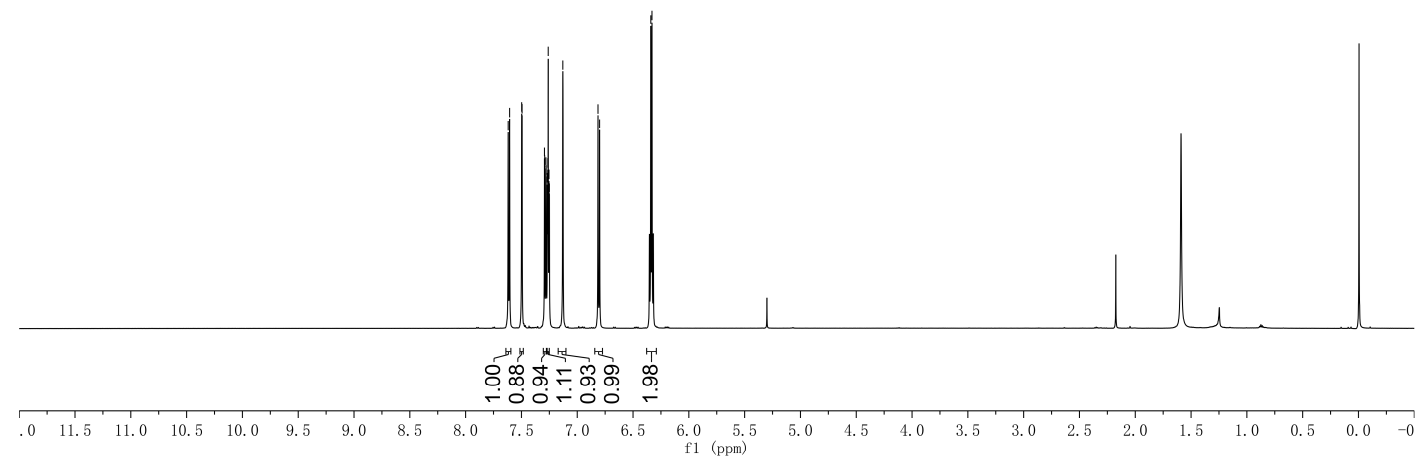

zj2008124

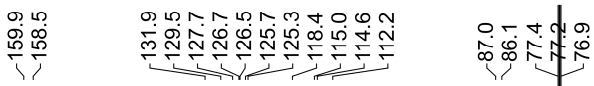<smiles>N#Cc1ccc2c(c1)OC1OC2Oc2ccc(Cl)cc21</smiles>

${ }^{13} \mathrm{C} \mathrm{NMR} \mathrm{(150} \mathrm{MHz,} \mathrm{CDCl}_{3}$ )

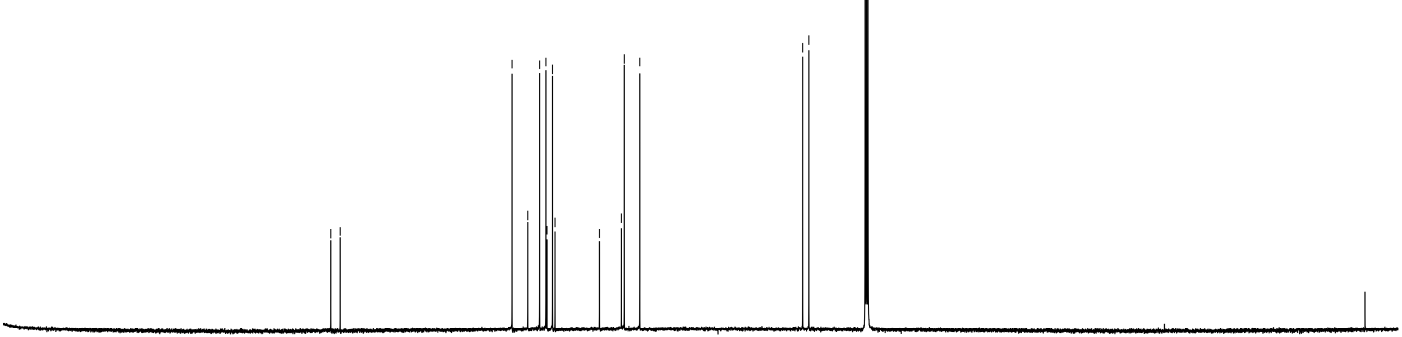

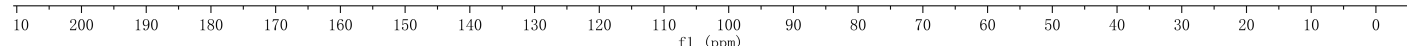



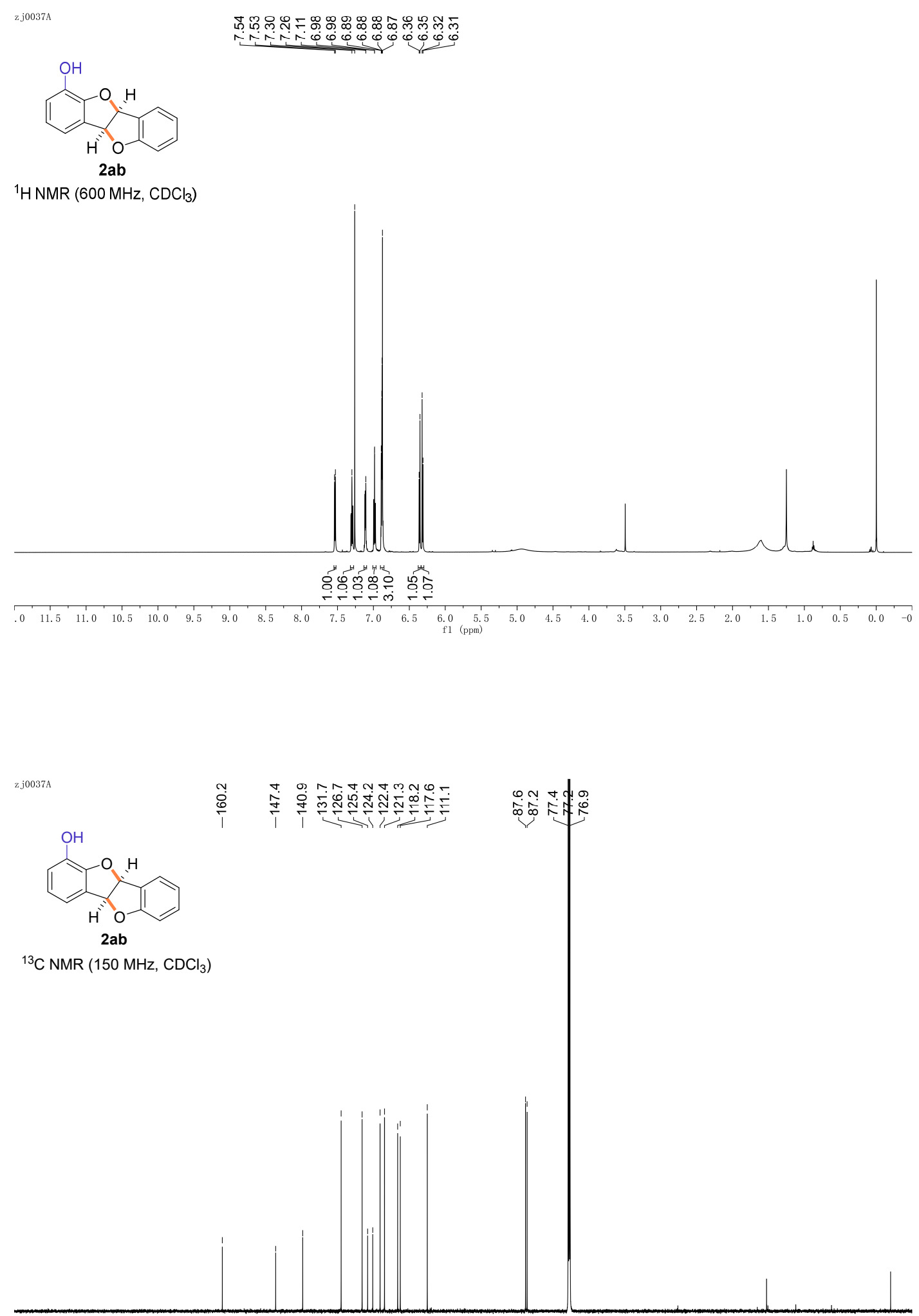

\begin{tabular}{llllllllllll}
\hline 10 & 200 & 190 & 180 & 170 & 160 & 150 & 140 & 130 & 120 & 110 & $\begin{array}{r}100 \\
\mathrm{f} 1\end{array}$ \\
$(\mathrm{ppm})$
\end{tabular} 

zj200892

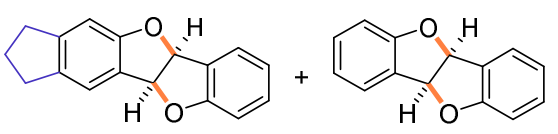

2ac and 2a (5:3)

${ }^{1} \mathrm{H} \mathrm{NMR}\left(600 \mathrm{MHz}, \mathrm{CDCl}_{3}\right)$

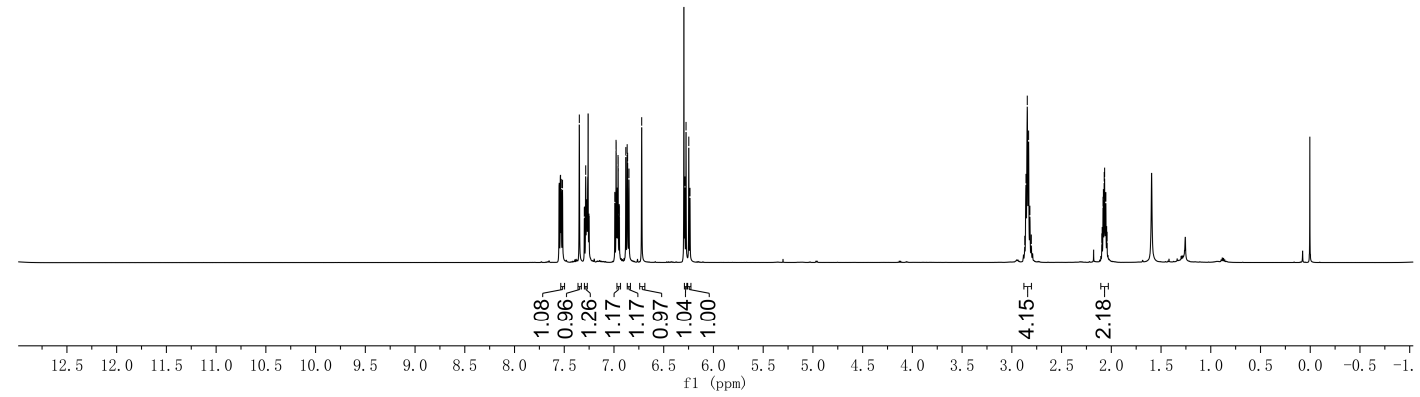

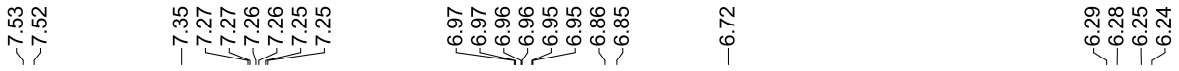

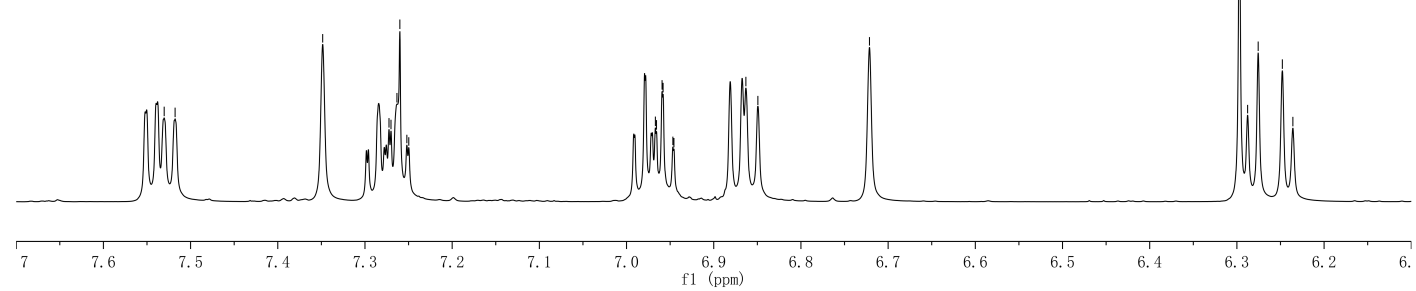

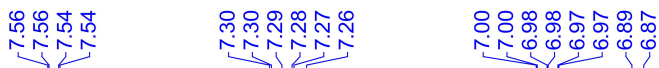
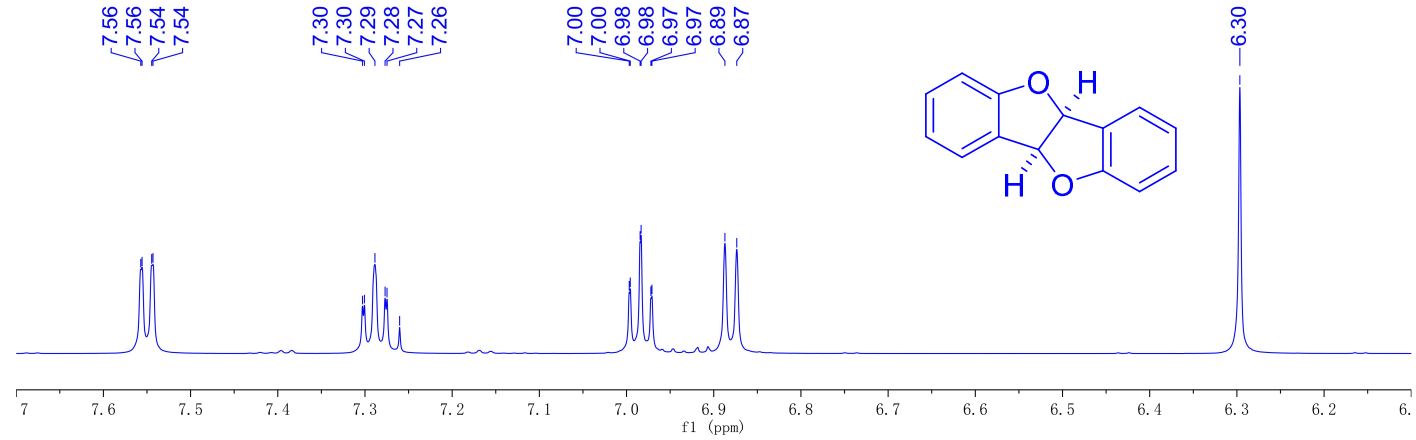

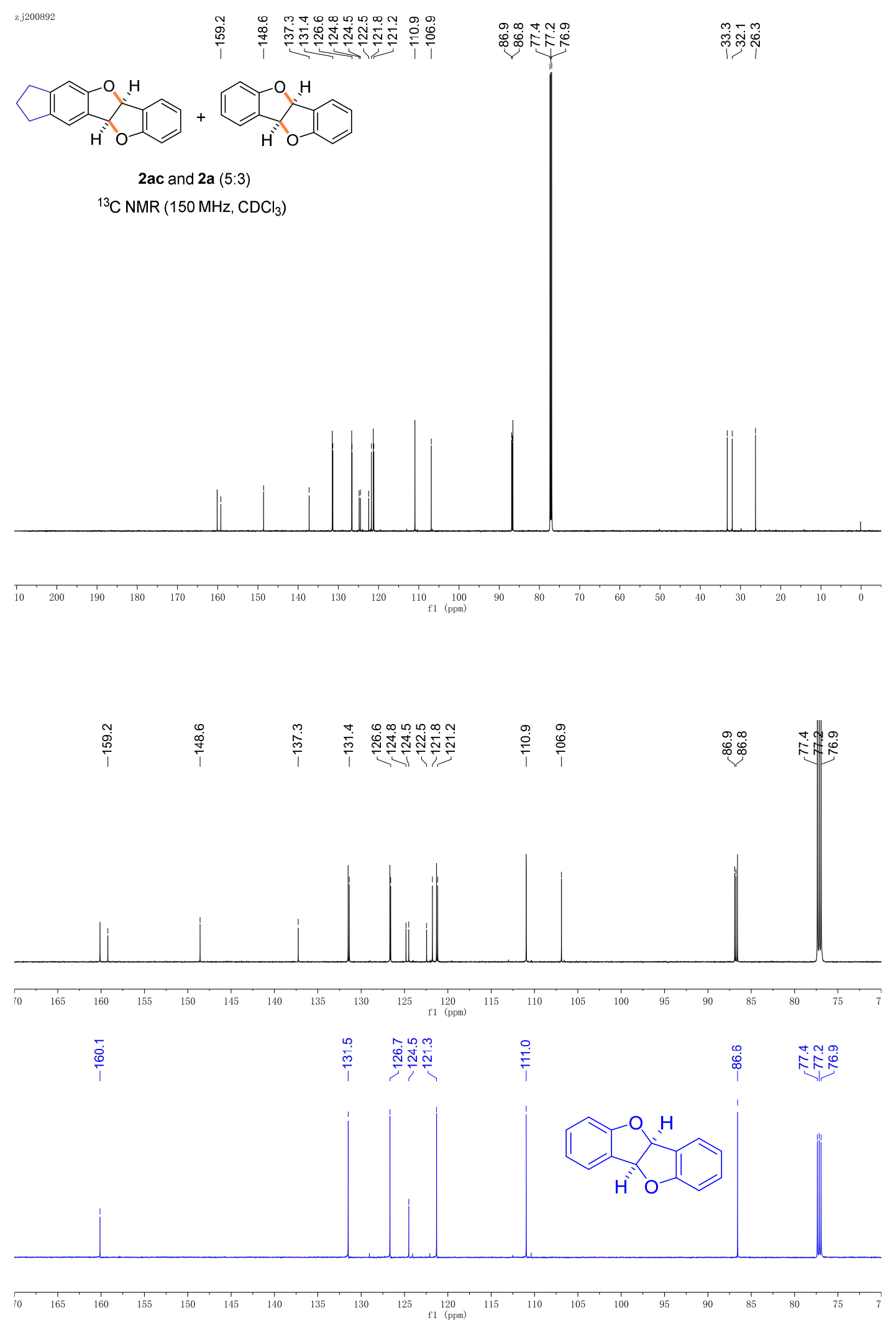
ZJ2008134

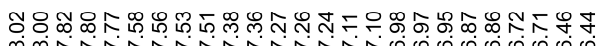

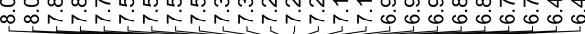

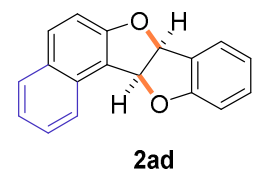

${ }^{1} \mathrm{H} \mathrm{NMR}\left(500 \mathrm{MHz}, \mathrm{CDCl}_{3}\right)$

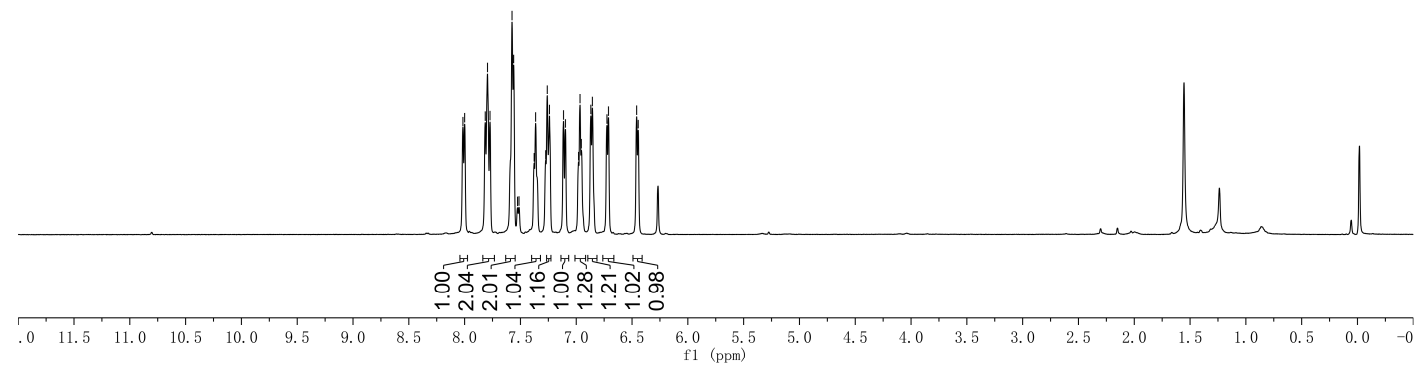

ZJ2008134
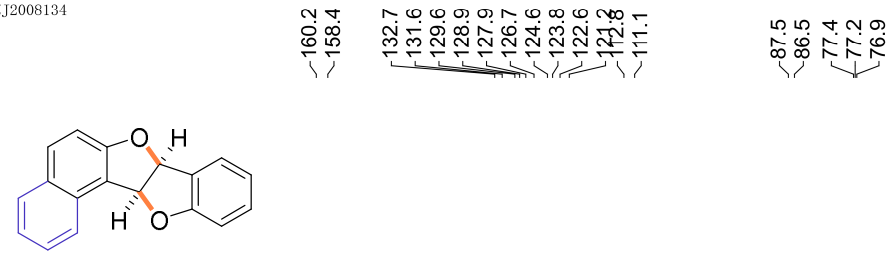

2ad

${ }^{13} \mathrm{C} \mathrm{NMR}\left(125 \mathrm{MHz}, \mathrm{CDCl}_{3}\right)$

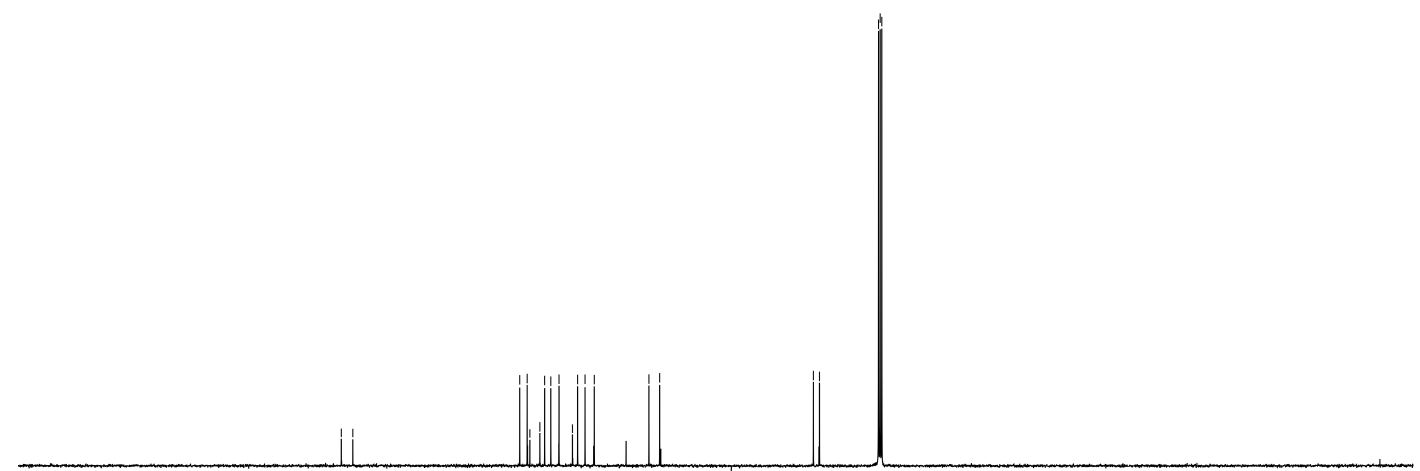

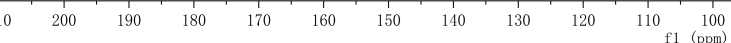


zj2008225

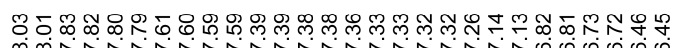

on

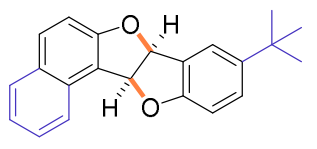

2ae

${ }^{1} \mathrm{H}$ NMR $\left(600 \mathrm{MHz}, \mathrm{CDCl}_{3}\right)$
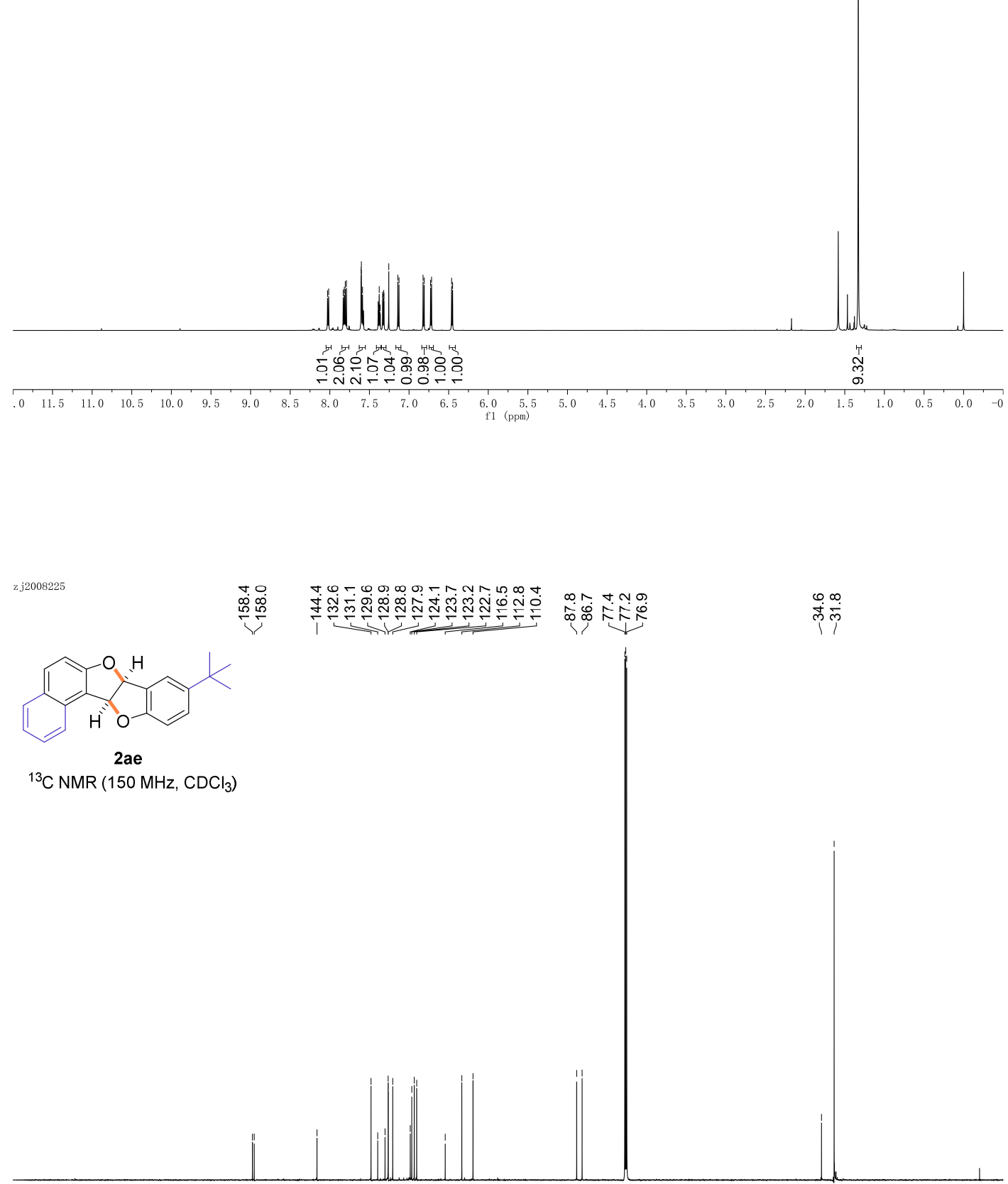

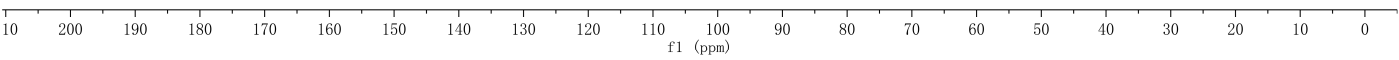


S001191-4A

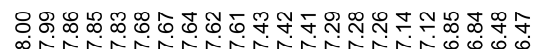

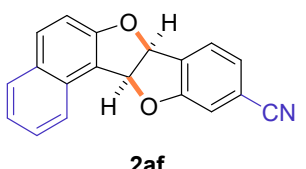

${ }^{1} \mathrm{H}$ NMR $\left(600 \mathrm{MHz}, \mathrm{CDCl}_{3}\right)$

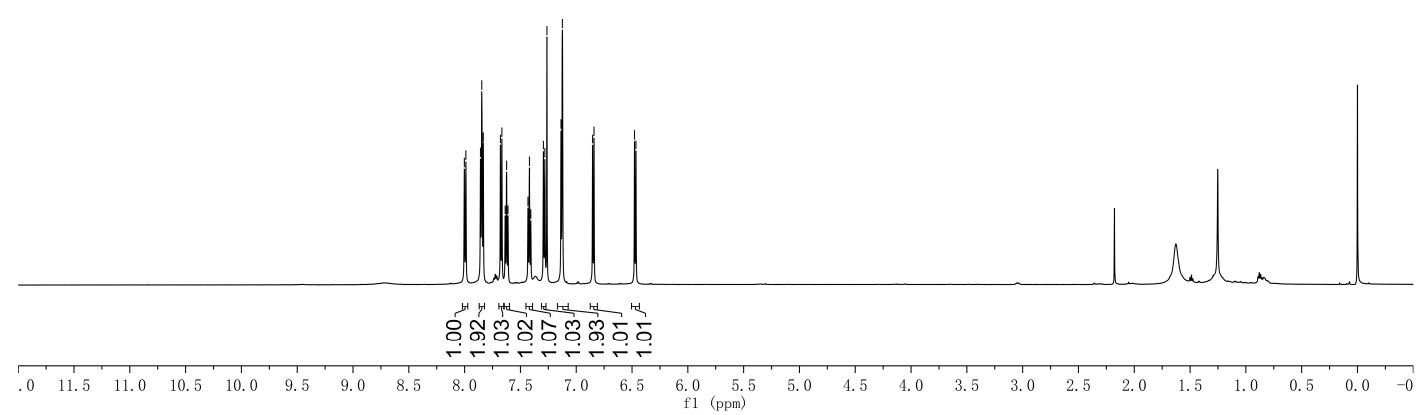

S001191-4A

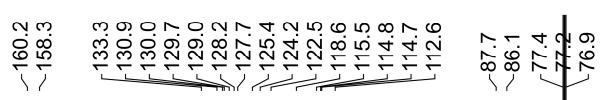

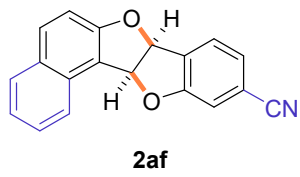

${ }^{13} \mathrm{C}$ NMR $\left(150 \mathrm{MHz}, \mathrm{CDCl}_{3}\right)$

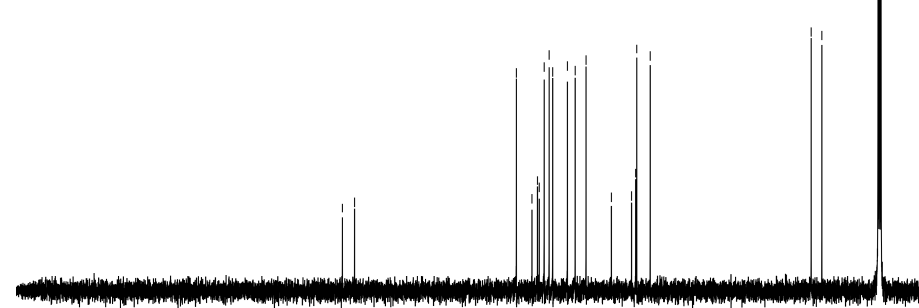

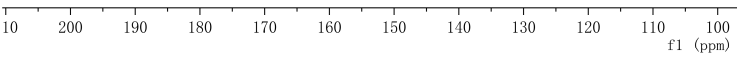


S001191-3A

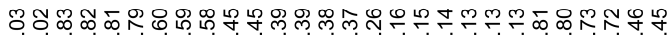

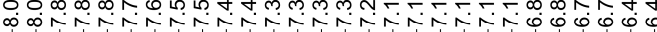

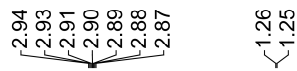

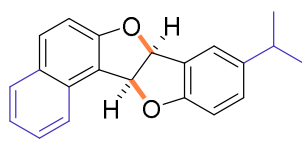

2ag

${ }^{1} \mathrm{H}$ NMR $\left(600 \mathrm{MHz}, \mathrm{CDCl}_{3}\right)$

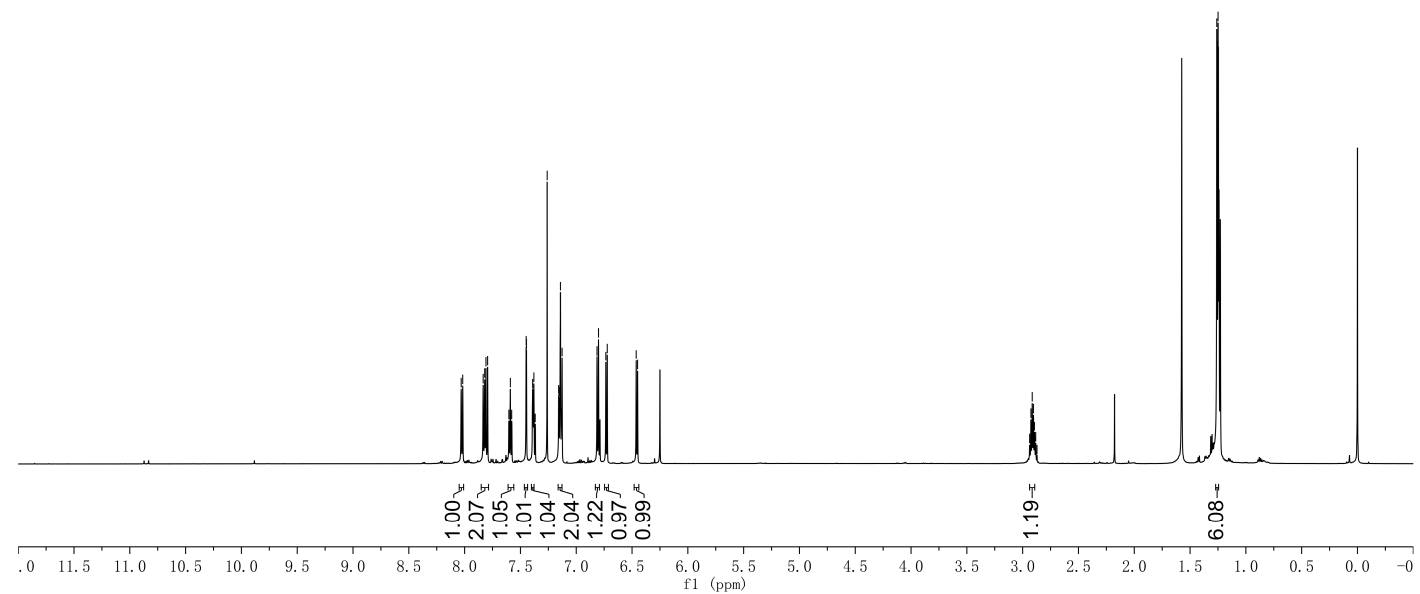

S001191-3A

每

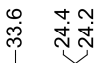

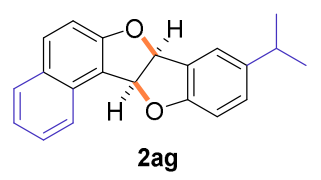

${ }^{13} \mathrm{C}$ NMR $\left(150 \mathrm{MHz}, \mathrm{CDCl}_{3}\right)$

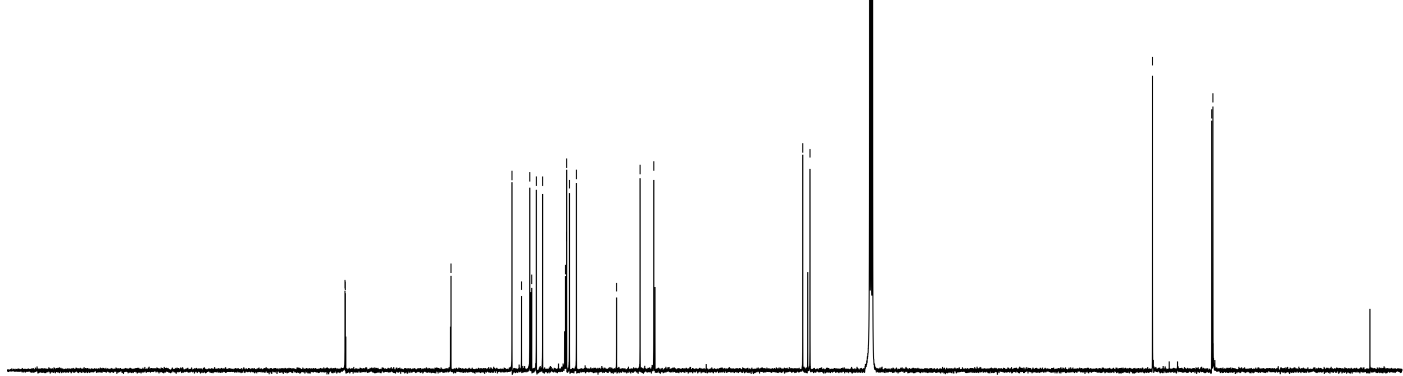

\begin{tabular}{llllllllllll}
\hline 10 & 200 & 190 & 180 & 170 & 160 & 150 & 140 & 130 & 120 & 110 & 100 \\
$\mathrm{f} 1$ & $1 \mathrm{pmm})$
\end{tabular} 

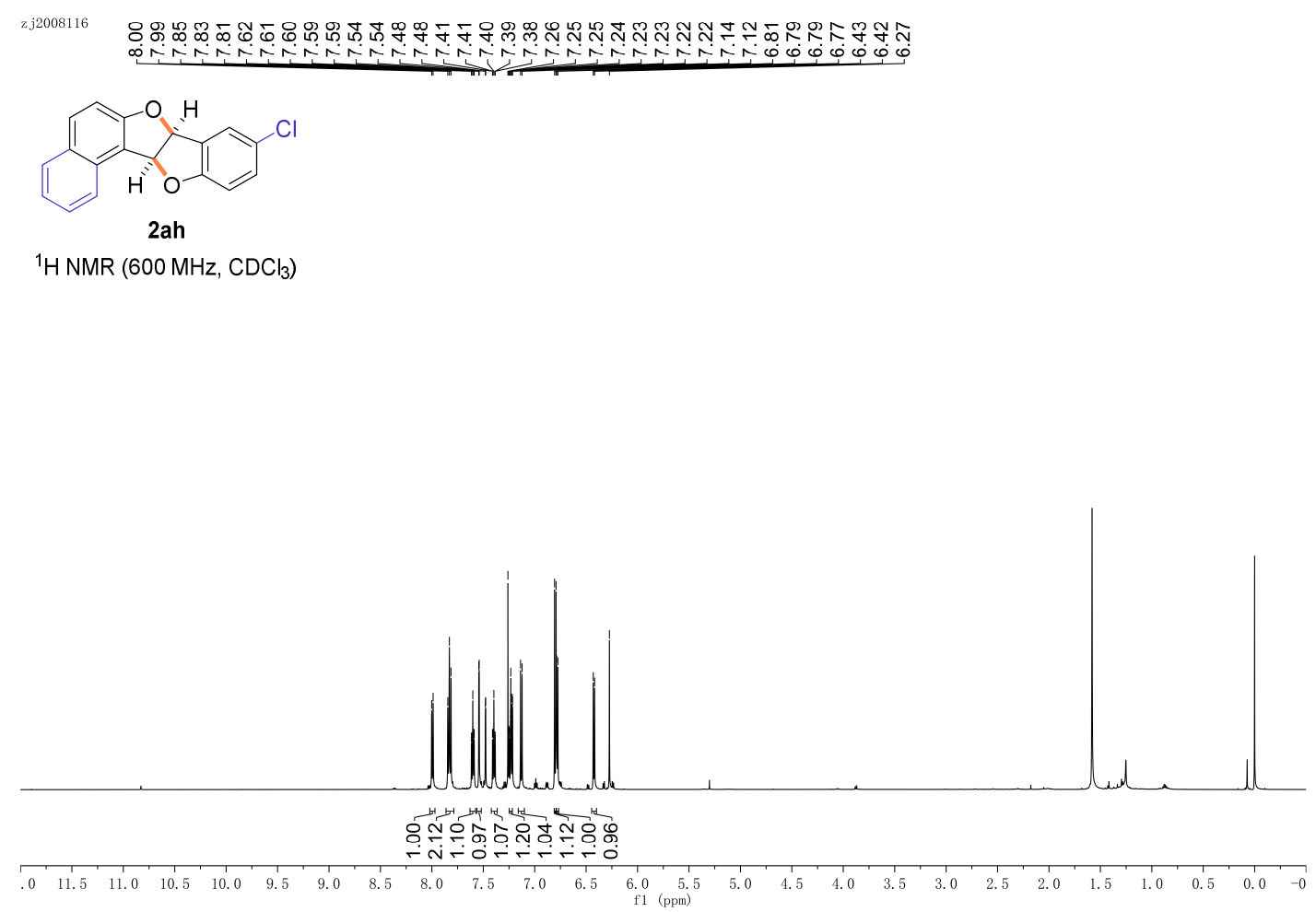

zj2008116
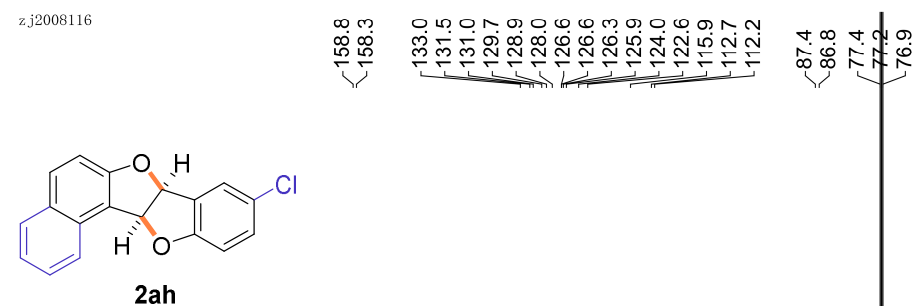

${ }^{13} \mathrm{C} \mathrm{NMR}\left(150 \mathrm{MHz}, \mathrm{CDCl}_{3}\right)$

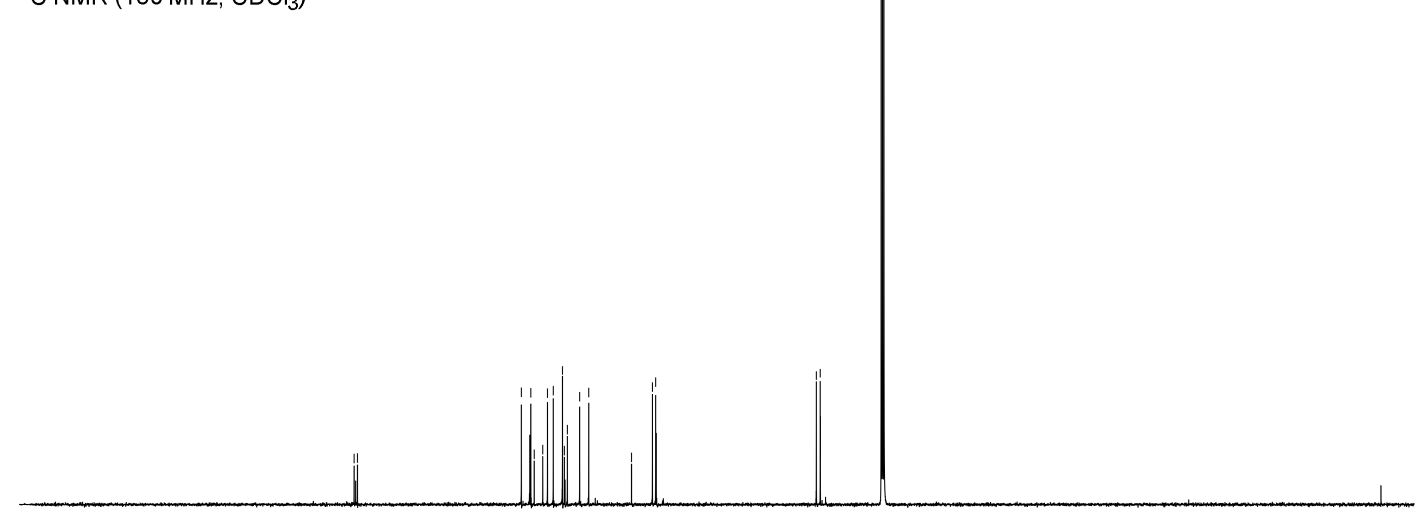

\begin{tabular}{llllllllllll}
\hline 10 & 200 & 190 & 180 & 170 & 160 & 150 & 140 & 130 & 120 & 110 & 100 \\
$\mathrm{f} 1$ & $1 \mathrm{ppm})$
\end{tabular} 
2.20081184

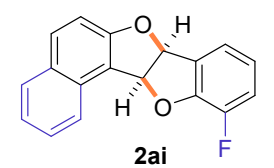

${ }^{1} \mathrm{H} \mathrm{NMR}\left(600 \mathrm{MHz}, \mathrm{CDCl}_{3}\right)$

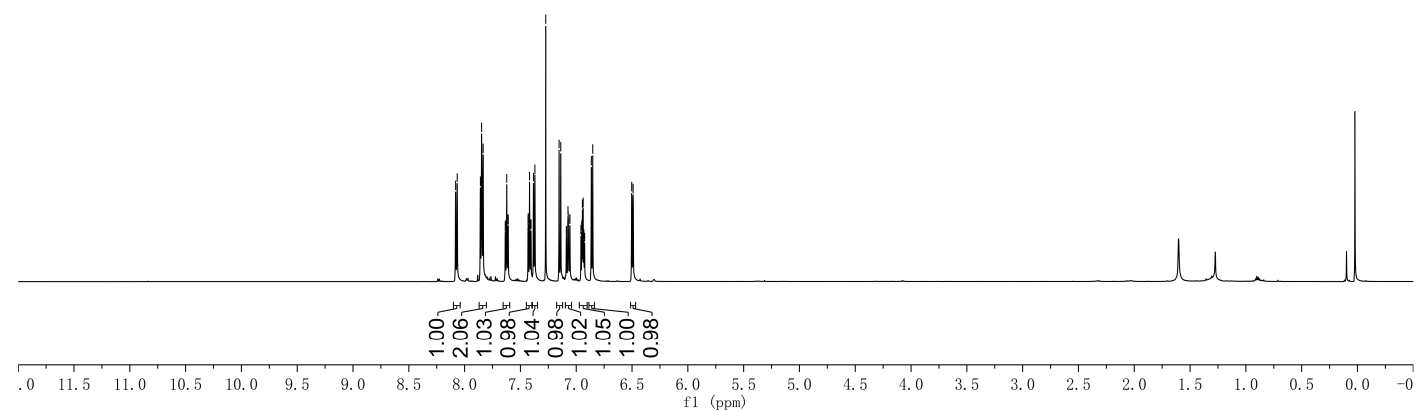

zj2008118A

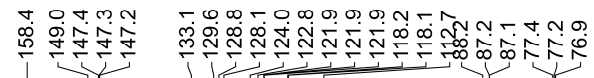

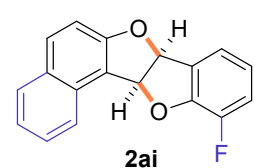

${ }^{13} \mathrm{C} \mathrm{NMR}\left(150 \mathrm{MHz}, \mathrm{CDCl}_{3}\right)$

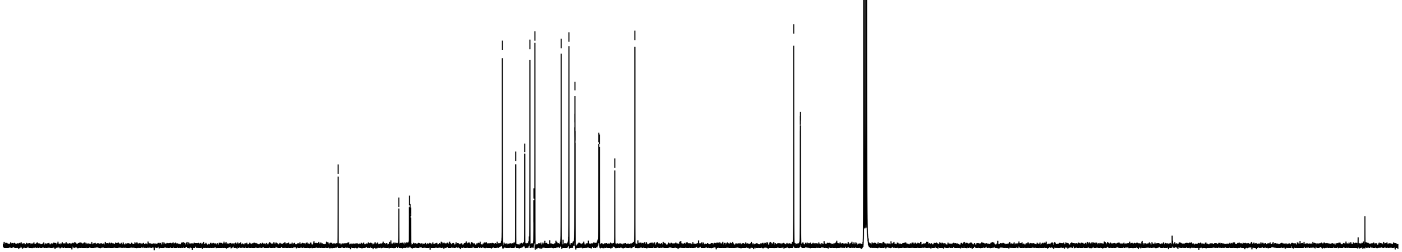

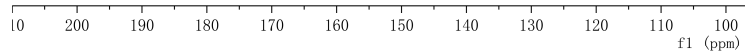


zj2008118AF

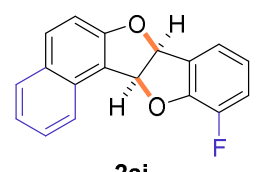

2ai

$\left.{ }^{19} \mathrm{~F} \mathrm{NMR} \mathrm{(471} \mathrm{MHz,} \mathrm{CDCl}_{3}\right)$

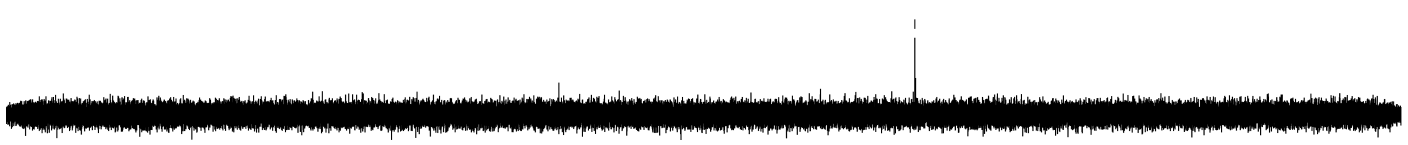

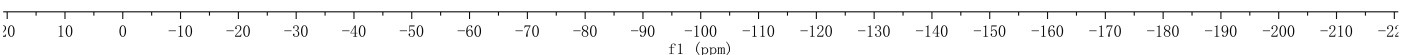


S001195-bod

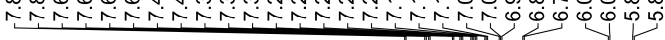

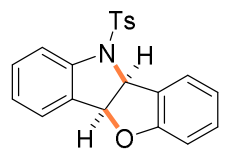

2aj

${ }^{1} \mathrm{H} \mathrm{NMR}\left(600 \mathrm{MHz}, \mathrm{CDCl}_{3}\right)$

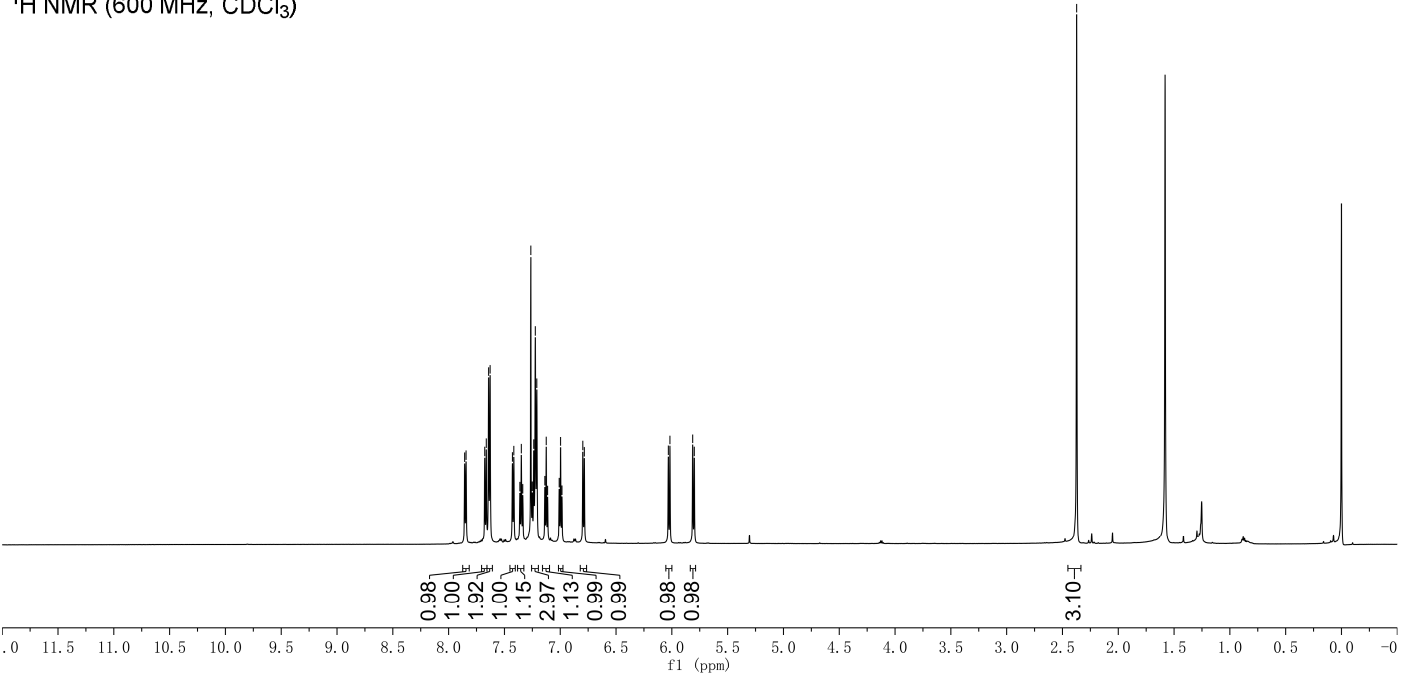

S001195-1A
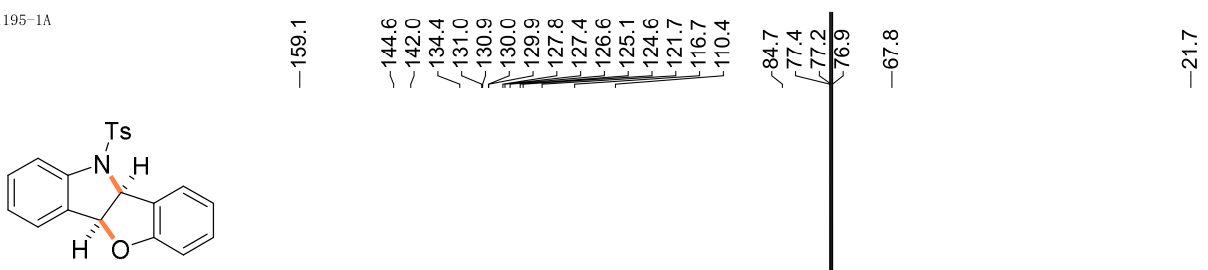

2aj

${ }^{13} \mathrm{C}$ NMR $\left(150 \mathrm{MHz}, \mathrm{CDCl}_{3}\right)$

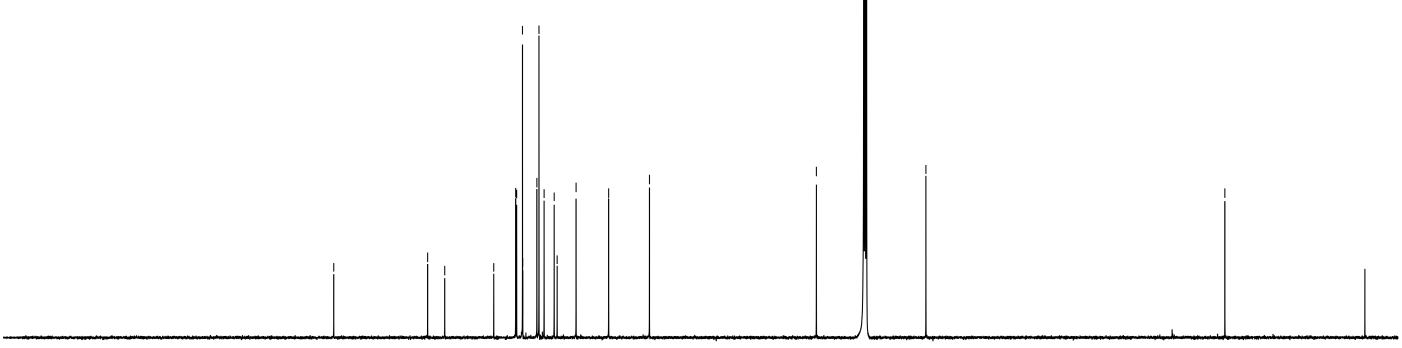

\begin{tabular}{llllllllllll}
\hline 10 & 200 & 190 & 180 & 170 & 160 & 150 & 140 & 130 & 120 & 110 & 100 \\
$\mathrm{f} 1$ & $1 \mathrm{pm})$
\end{tabular} 


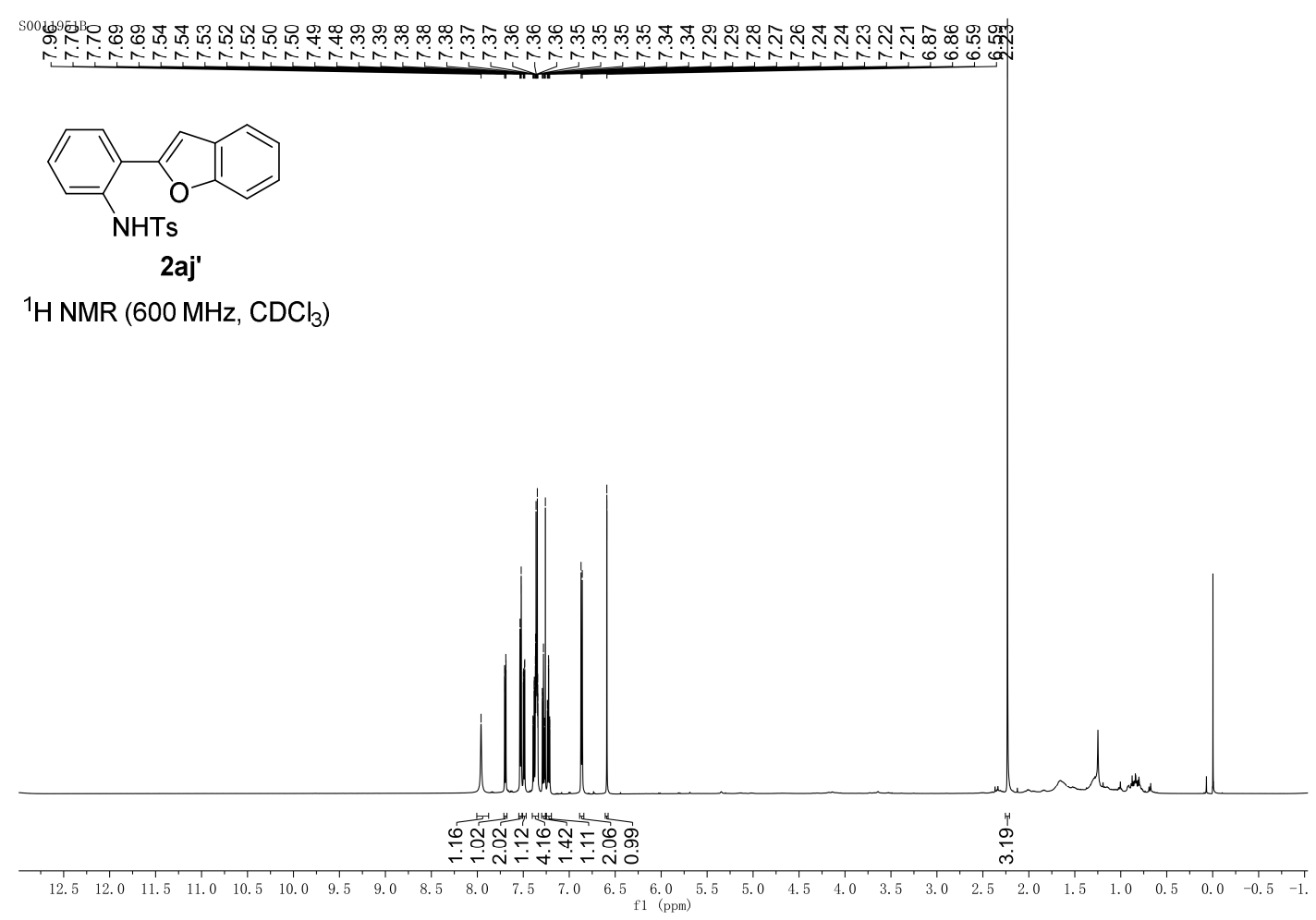

S0011951B

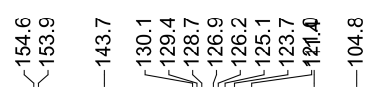

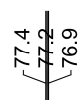

$\stackrel{\varphi}{\grave{i}}$

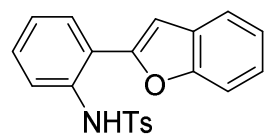

2aj'

${ }^{13} \mathrm{C} \mathrm{NMR}\left(150 \mathrm{MHz}, \mathrm{CDCl}_{3}\right)$

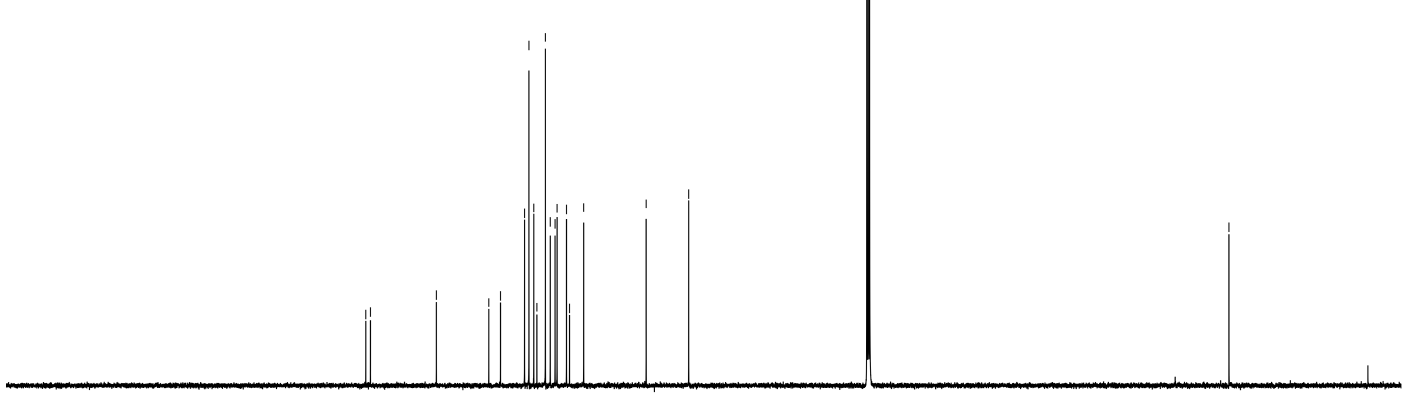

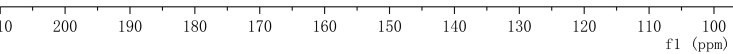




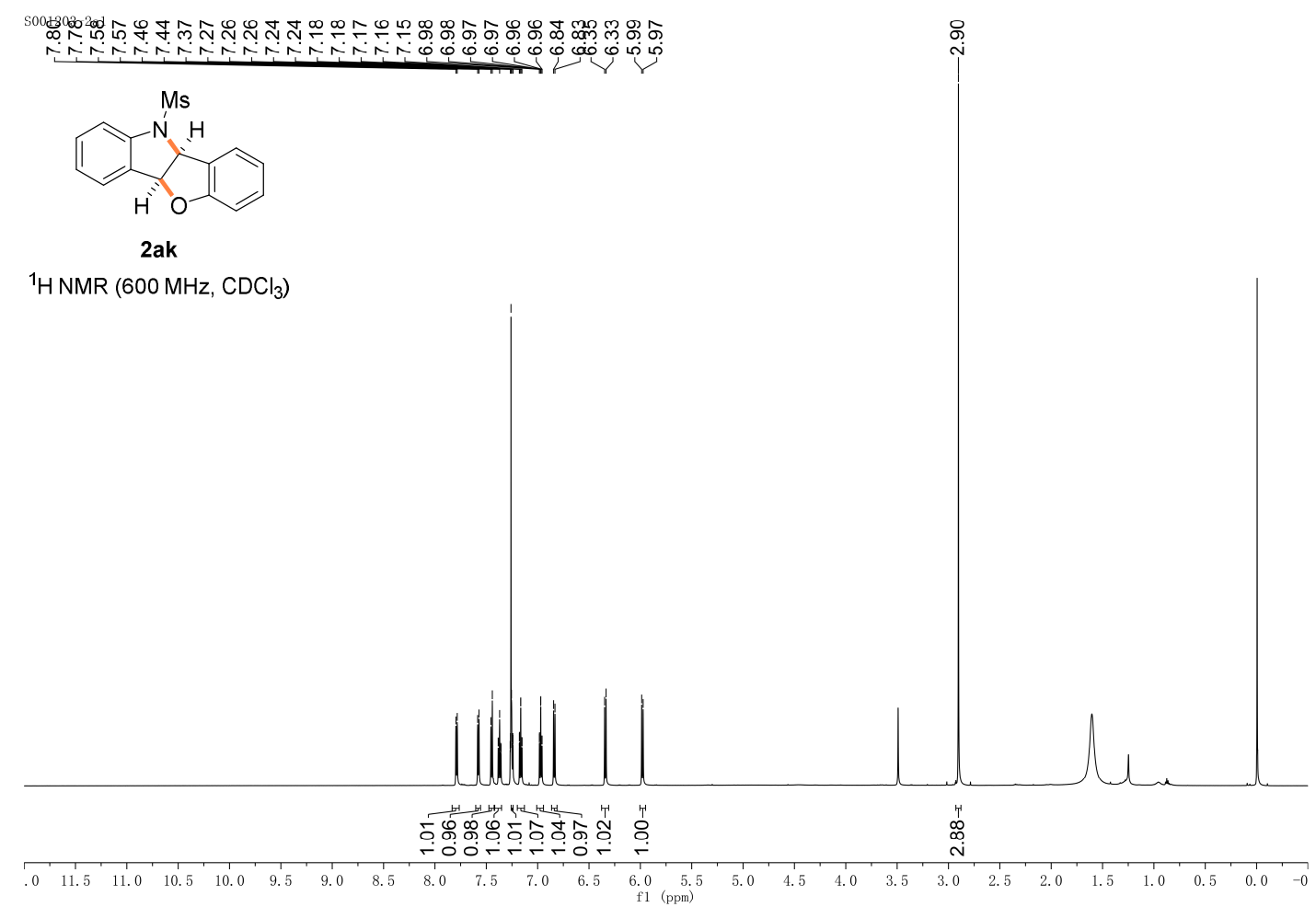

S001203-2a1
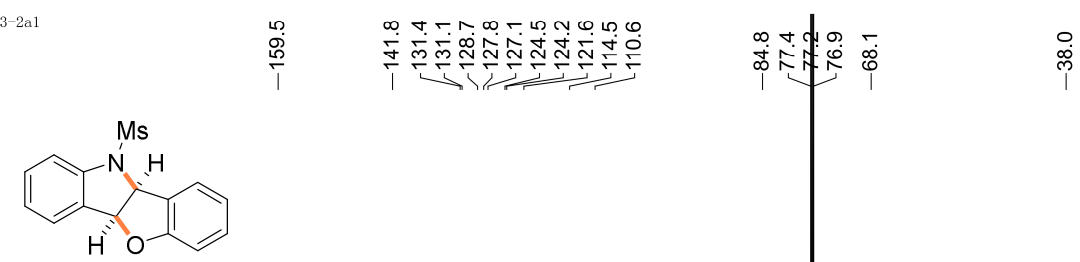

2ak

${ }^{13} \mathrm{C}$ NMR $\left(150 \mathrm{MHz}, \mathrm{CDCl}_{3}\right)$

\section{CNMR (15OMHz, $\left.\mathrm{CDCl}_{3}\right)$}

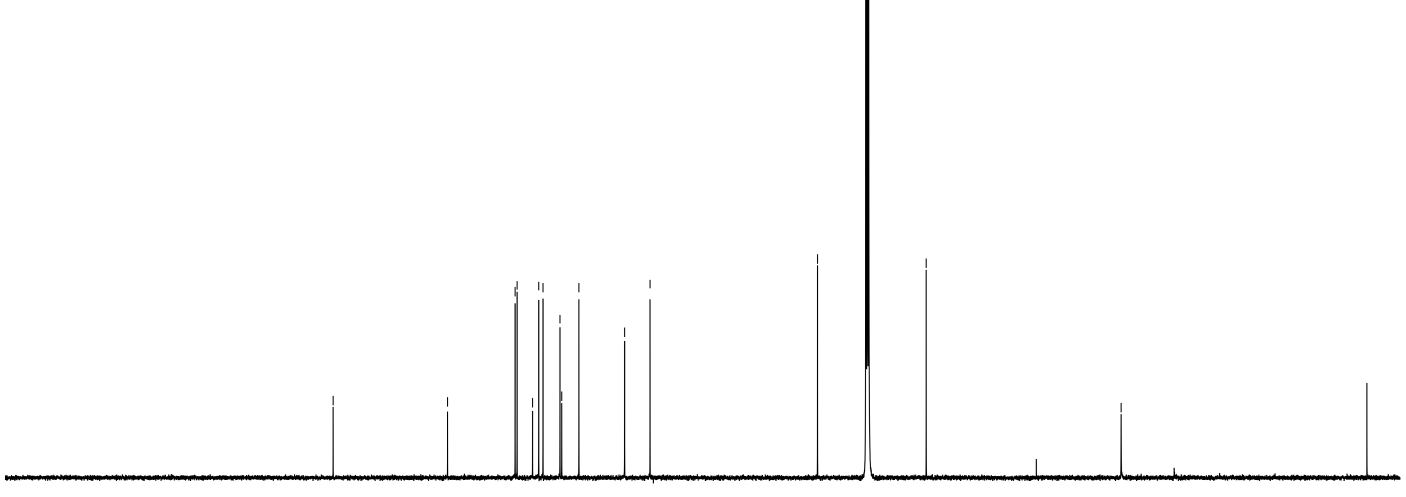

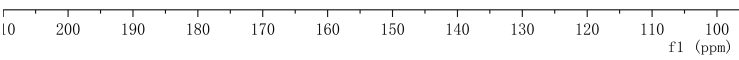




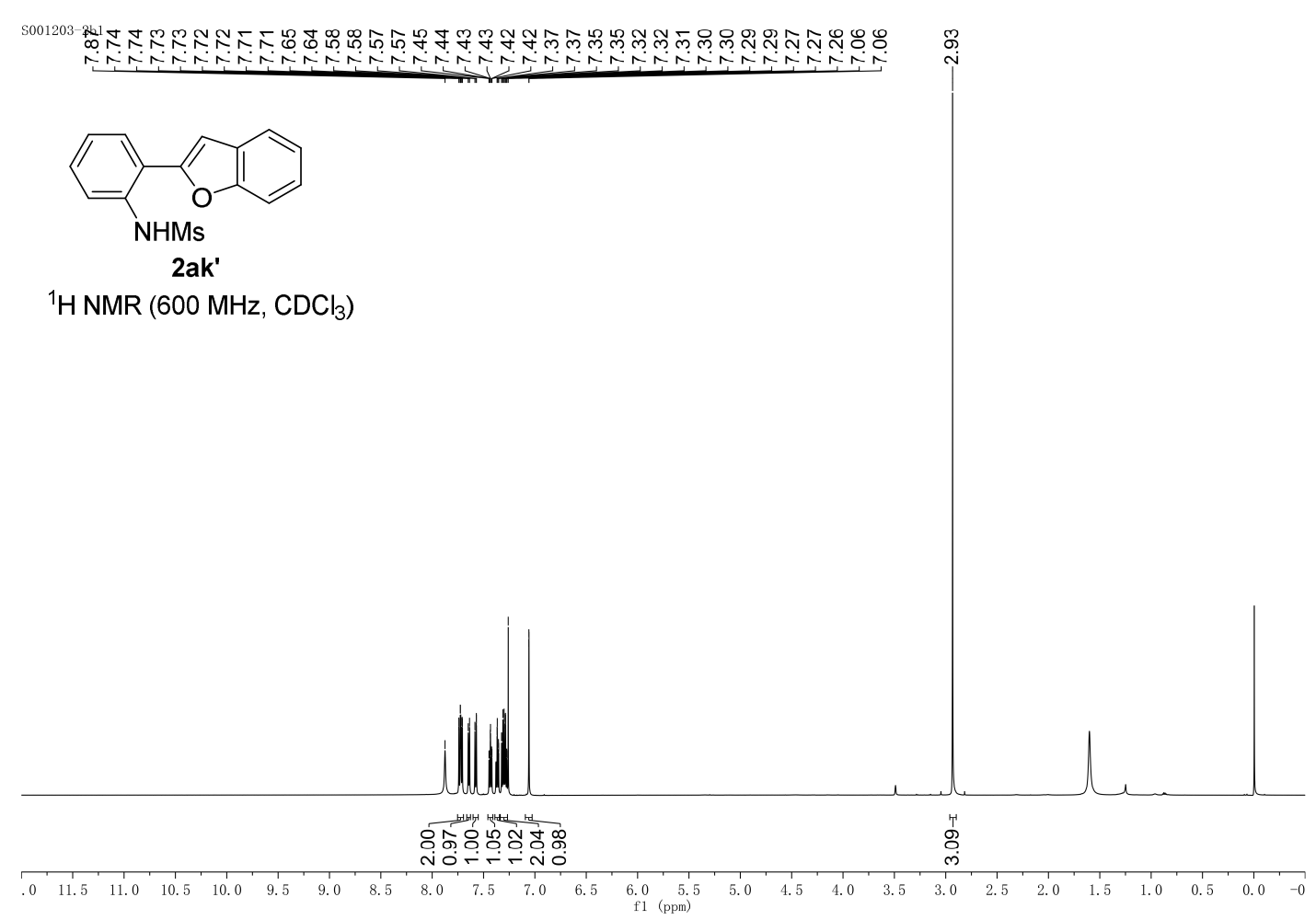

S001203-2b1

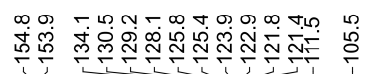

违

$\stackrel{\oplus}{\oplus !}$

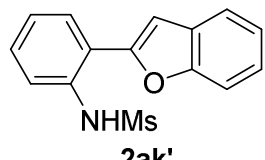

2ak'

${ }^{13} \mathrm{C}$ NMR $\left(150 \mathrm{MHz}, \mathrm{CDCl}_{3}\right)$

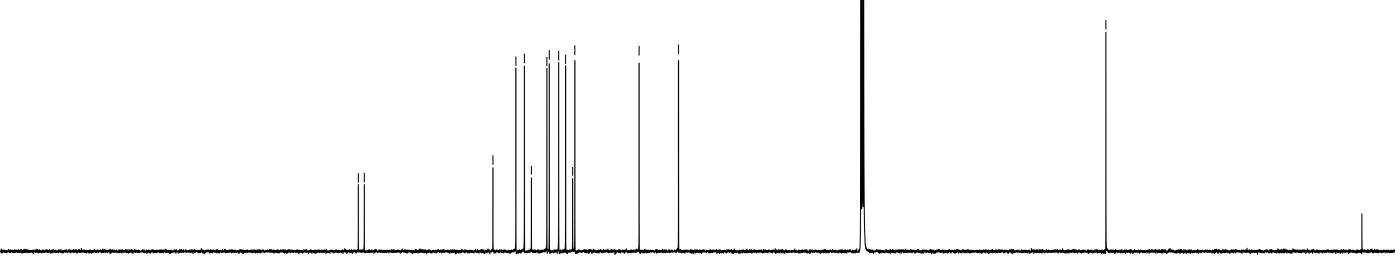

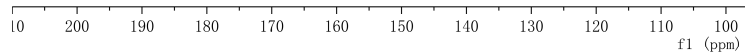




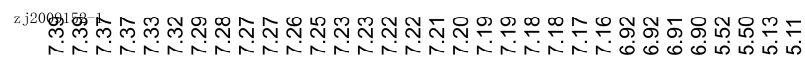

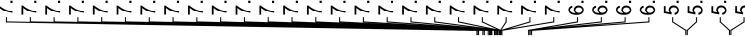

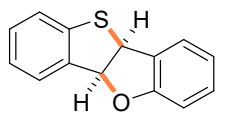

2al

${ }^{1} \mathrm{H} \mathrm{NMR}\left(600 \mathrm{MHz}, \mathrm{CDCl}_{3}\right)$

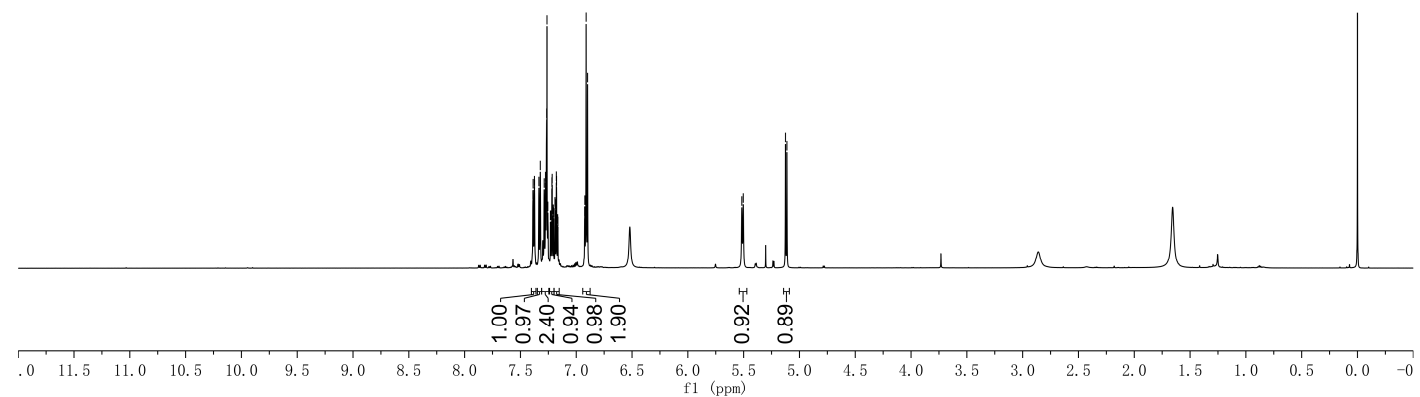

z.j2009152-1

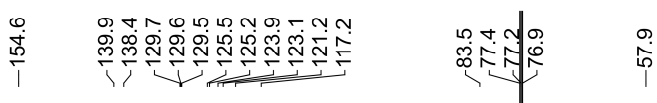

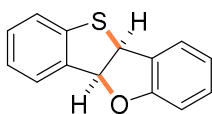

2al

${ }^{13} \mathrm{C} \mathrm{NMR}\left(150 \mathrm{MHz}, \mathrm{CDCl}_{3}\right)$

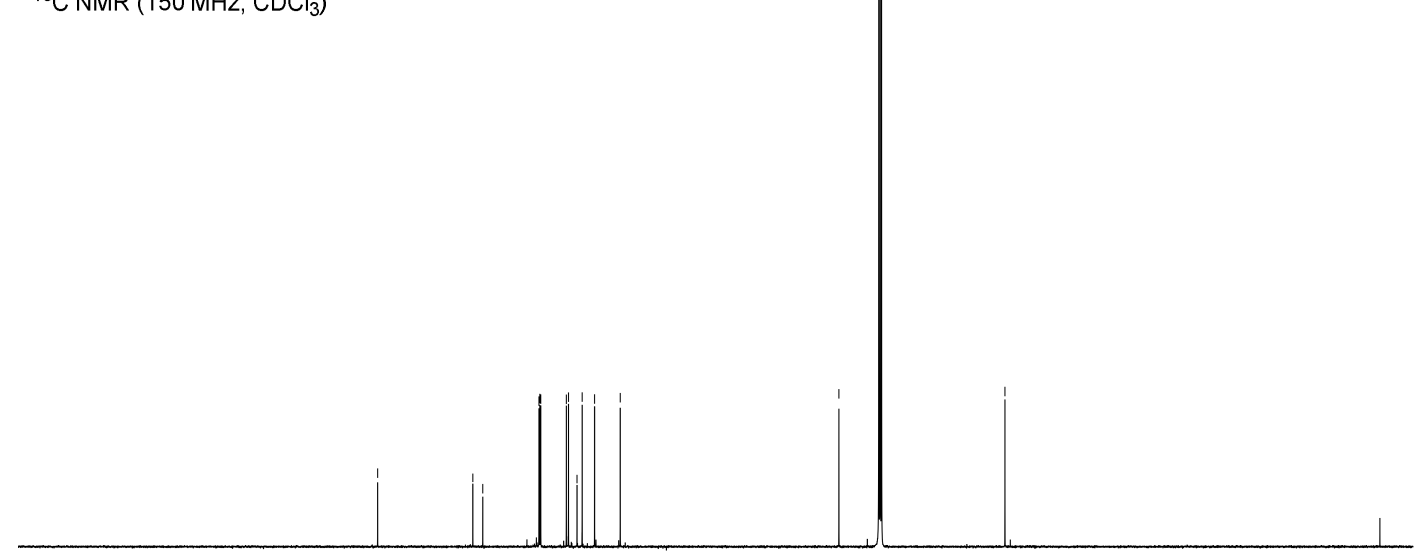

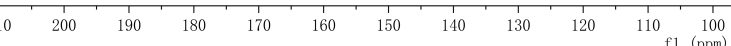



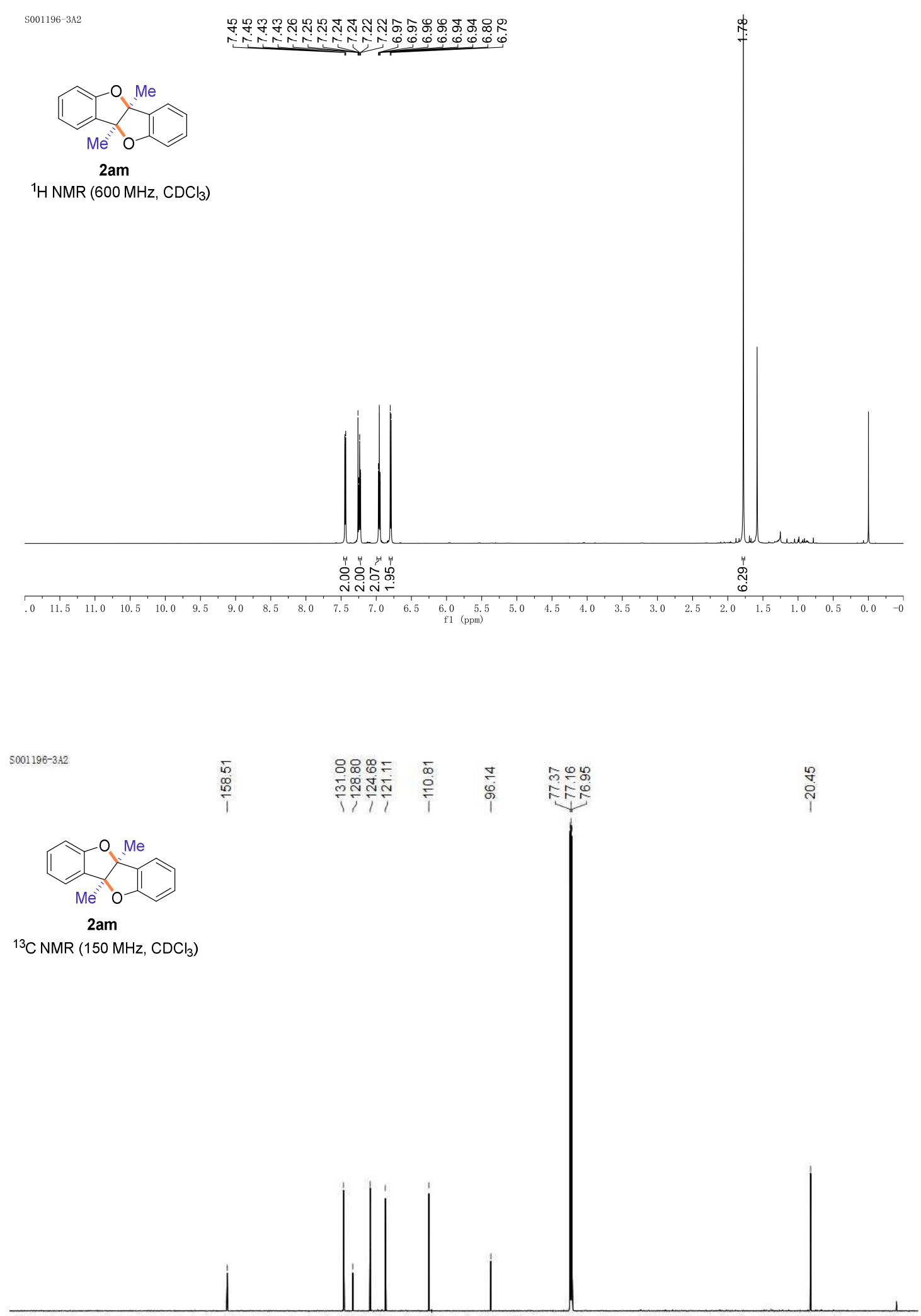

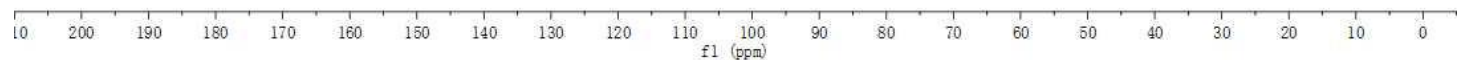



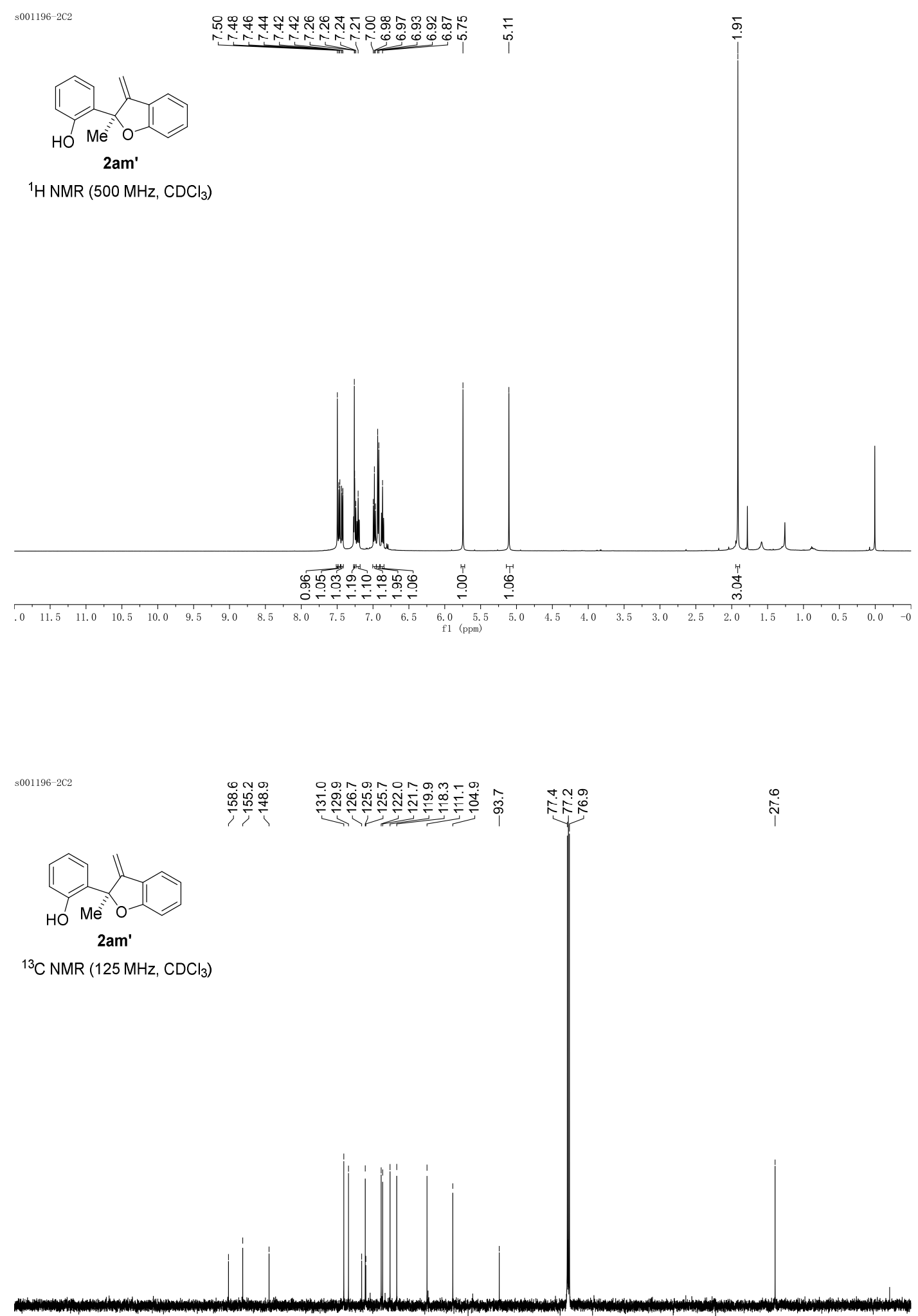

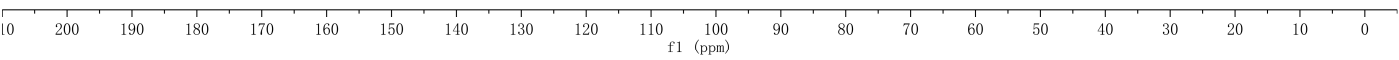




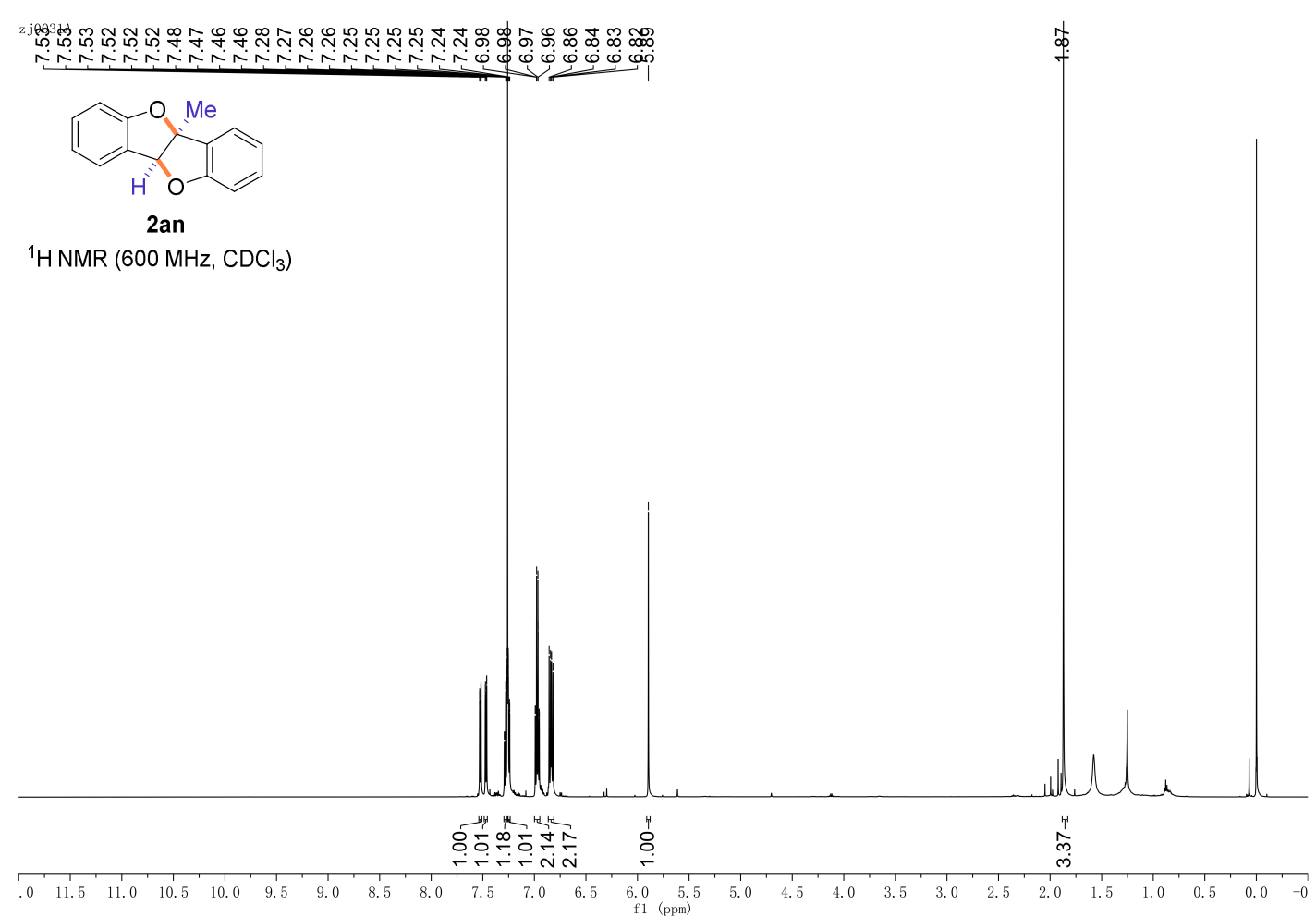

zj0031A
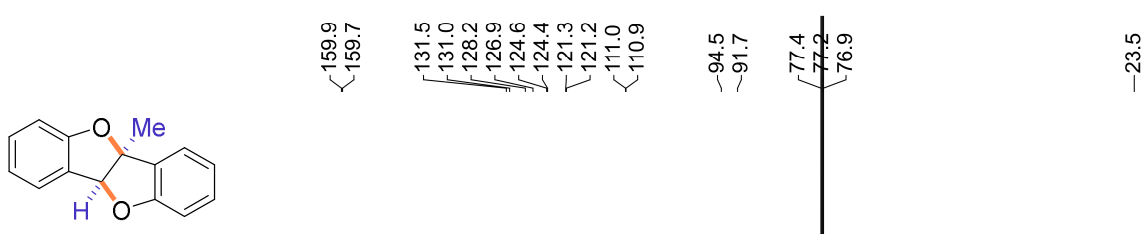

2an

${ }^{13} \mathrm{C}$ NMR $\left(150 \mathrm{MHz}, \mathrm{CDCl}_{3}\right)$

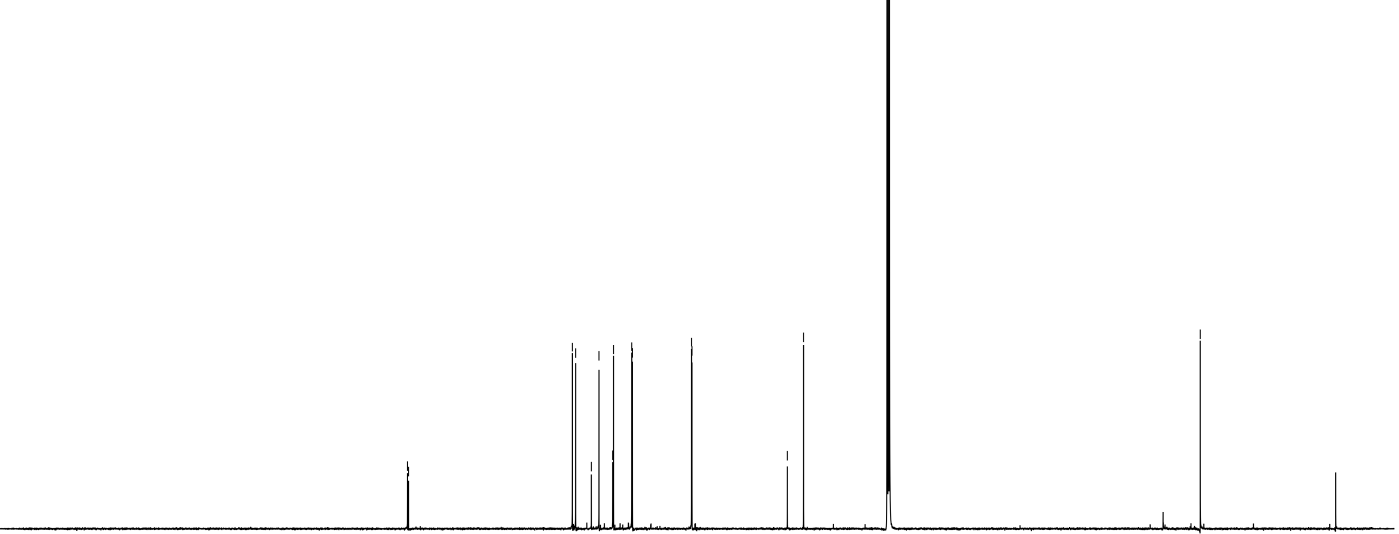

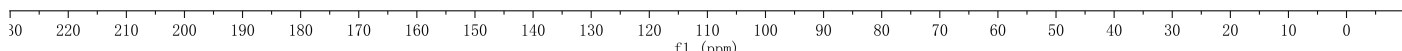




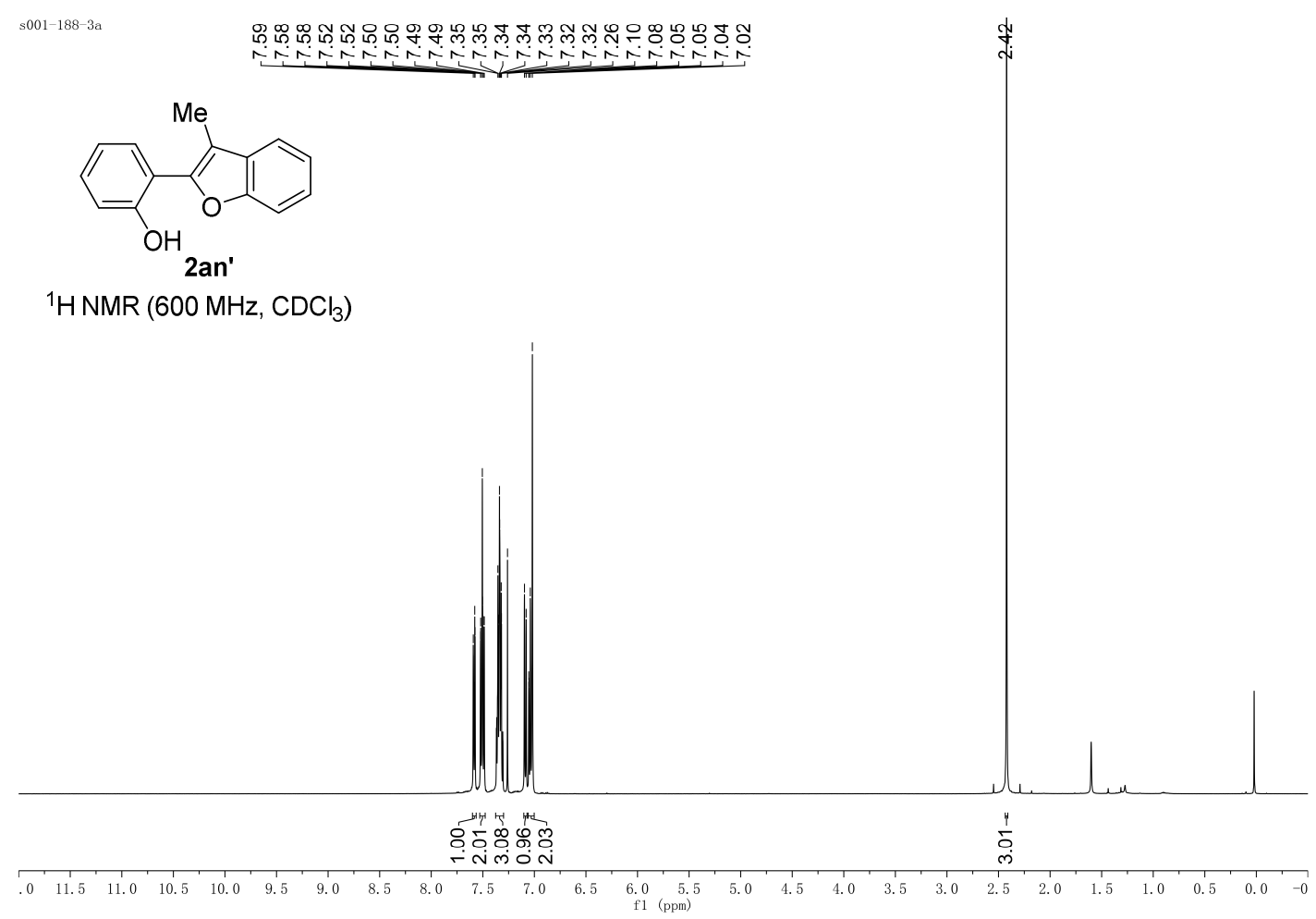

s001-188-3a

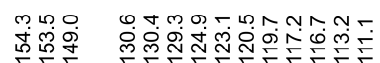

紊少

के

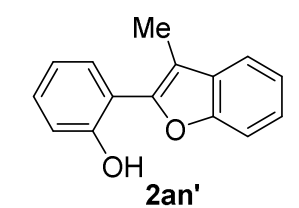

${ }^{13} \mathrm{C} \mathrm{NMR}\left(150 \mathrm{MHz}, \mathrm{CDCl}_{3}\right)$

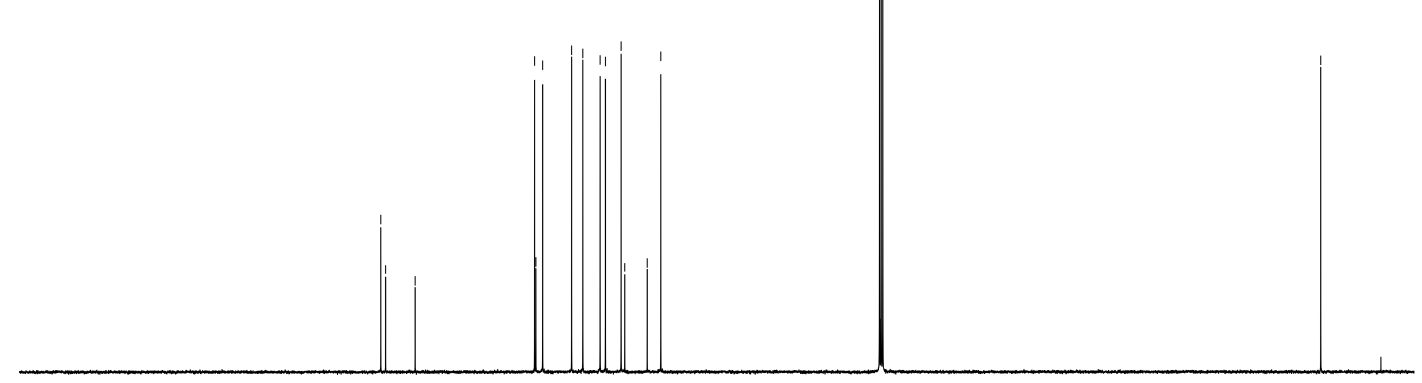

\begin{tabular}{llllllllllll}
\hline 10 & 200 & 190 & 180 & 170 & 160 & 150 & 140 & 130 & 120 & 110 & 100 \\
$\mathrm{f} 1$ & $\begin{array}{l}1 \\
(\mathrm{ppm})\end{array}$
\end{tabular} 